Electronic Supporting Information for:

\title{
Substituent Effects on Exchange Coupling and Magnetic Relaxation in 2,2'-Bipyrimidine Radical-Bridged Dilanthanide Complexes
}

Colin A. Gould, ${ }^{\dagger}$ Edward Mu, ${ }^{\dagger}$ Veacheslav Vieru, ${ }^{\ddagger}$ Lucy E. Darago, ${ }^{\dagger}$ Khetpakorn Chakarawet, ${ }^{\dagger}$ Miguel I. Gonzalez, ${ }^{\dagger}$ Selvan Demir, ${ }^{\dagger \#}$ and Jeffrey R. Long ${ }^{\dagger} \|_{*}$

${ }^{\dagger}$ Department of Chemistry and "Department of Chemical and Biomolecular Engineering, University of California, Berkeley, Berkeley, California 94720, United States

$\$$ Theory of Nanomaterials Group, Katholieke Universiteit Leuven, Celestijnenlaan 200F, 3001 Leuven, Belgium

\#Department of Chemistry, Michigan State University, East Lansing, Michigan 48824, United States

${ }^{\perp}$ Materials Sciences Division, Lawrence Berkeley National Laboratory, Berkeley, California 94720, United States

${ }^{\S}$ Current Address: Maastricht Science Programme, Faculty of Science and Engineering, Maastricht University, Maastricht, the Netherlands 


\section{Table of Contents}

Section S1. Additional synthetic procedures $\quad$ S3

Section S2. NMR spectroscopy $\quad$ S4

Section S3. Infrared spectroscopy $\quad \mathrm{S} 12$

$\begin{array}{ll}\text { Section S4. X-ray crystallography data refinement details } & \text { S19 }\end{array}$

Section S5. Magnetic measurements $\quad$ S31

$\begin{array}{ll}\text { Section S5.1 De magnetic susceptibility data } & \text { S31 }\end{array}$

Section S5.2 Ac magnetic susceptibility data $\quad$ S40

Section S5.3 De magnetic relaxation data $\quad$ S52

$\begin{array}{ll}\text { Section S5.4 Analysis of magnetic relaxation dynamics } & \text { S64 }\end{array}$

$\begin{array}{ll}\text { Section } S 5.5 \text { Empirical correlation between } U_{\text {eff }} \text { and } J_{\mathrm{Gd}-\mathrm{rad}} & \mathrm{S} 72\end{array}$

$\begin{array}{ll}\text { Section S5.6 Magnetic hysteresis data } & \text { S77 }\end{array}$

Section S6. Calculations $\quad$ S82

$\begin{array}{ll}\text { Section S7. References } & \text { S88 }\end{array}$ 


\section{Section S1. Additional synthetic procedures}

$\left[\left(\mathbf{D y C p}_{2}\right)_{2}\left(\boldsymbol{\mu}-\mathbf{5}, \mathbf{5}^{\prime}-\left(\mathrm{NMe}_{2}\right)_{2} \mathbf{b p y m}\right)\right]\left(\mathbf{B P h}_{4}\right)$ (1-Dy). Analogous to the synthesis of 1-Gd, [Cp* $\left.{ }_{2} \mathrm{Dy}\right]\left(\mathrm{BPh}_{4}\right)(53.3 \mathrm{mg}, 0.0707 \mathrm{mmol})$ was stirred in toluene $(4 \mathrm{~mL})$ with $\left(\mathbf{N M e}_{2}\right)_{2} \mathbf{b p y m}(8.7$ $\mathrm{mg}, 0.035 \mathrm{mmol}, 0.5$ equiv) and then $\mathrm{KC}_{8}(4.7 \mathrm{mg}, 0.035 \mathrm{mmol}, 0.5$ equiv) was added in THF ( 0.5 $\mathrm{mL}$ ). Insoluble solids were removed by centrifugation and then red, block-shaped crystals of 1-Dy were grown from a layered THF-toluene solution ( $2 \mathrm{~mL}$ THF, $2 \mathrm{~mL}$ toluene) cooled to $-30{ }^{\circ} \mathrm{C}$ for $48 \mathrm{~h}$ (27.2 mg, 54\% yield). Anal. Calcd. For $\mathrm{C}_{76} \mathrm{H}_{96} \mathrm{BN}_{6} \mathrm{Dy} 2: \mathrm{C}, 63.86 ; \mathrm{H}, 6.77 ; \mathrm{N}, 5.88$. Found: $\mathrm{C}$, $63.80 ; \mathrm{H}, 6.76 ; \mathrm{N}, 5.76$.

$\left[\left(\mathbf{T b C p}_{2}\right)_{2}\left(\mu-5,5^{\prime}-\left(\mathrm{NMe}_{2}\right)_{2} \mathbf{b p y m}\right)\right]\left(\mathbf{B P h}_{4}\right) \quad(\mathbf{1 - T b})$. Analogous to the synthesis of 1-Gd, $\left[\mathrm{Cp}_{2}{ }_{2} \mathrm{~Tb}\right]\left(\mathrm{BPh}_{4}\right)(61.3 \mathrm{mg}, 0.082 \mathrm{mmol})$ was stirred in toluene $(10 \mathrm{~mL})$ with $\left(\mathbf{N M e}_{2}\right)_{2} \mathbf{b p y m}(10$ $\mathrm{mg}, 0.041 \mathrm{mmol}, 0.5$ equiv) and then $\mathrm{KC}_{8}(5.5 \mathrm{mg}, 0.041 \mathrm{mmol}, 0.5$ equiv) was added in THF ( 0.5 $\mathrm{mL}$ ). Solvent was removed under reduced pressure, and 1,2-difluorobenzene (DFB, $2 \mathrm{~mL}$ ) was added to form a dark reddish-black solution. Insoluble solids were removed by filtration through diatomaceous earth and then red, block-shaped crystals of 1-Tb were grown from a layered DFBhexanes solution ( $2 \mathrm{~mL}$ DFB, $4 \mathrm{~mL}$ hexane) cooled to $-30{ }^{\circ} \mathrm{C}$ for 72 hours $(20 \mathrm{mg}, 17 \%$ yield). Anal. Calcd. For $\mathrm{C}_{76} \mathrm{H}_{96} \mathrm{BN}_{6} \mathrm{~Tb}_{2}$ : C, 64.18; H, 6.80; N, 5.91. Found: C, 64.01; H, 6.81; N, 6.13.

$\left[\left(\mathbf{D y C p}_{2}\right)_{2}\left(\boldsymbol{\mu - 5 , 5} \mathbf{5}^{\prime}-(\mathrm{OEt})_{2} \mathbf{b p y m}\right)\right]\left(\mathbf{B P h}_{4}\right)$ (2-Dy) Analogous to the synthesis of 1-Gd, $\left[\mathrm{Cp}_{2}{ }_{2} \mathrm{Dy}\right](\mathrm{BPh} 4)(35.5 \mathrm{mg}, 0.0471 \mathrm{mmol})$ was stirred in toluene $(4 \mathrm{~mL})$ with $(\mathbf{O E t})_{2} \mathbf{b p y m}(5.8$ $\mathrm{mg}, 0.024 \mathrm{mmol}, 0.5$ equiv) and then $\mathrm{KC}_{8}(3.2 \mathrm{mg}, 0.024 \mathrm{mmol}, 0.5$ equiv) was added in THF ( 0.5 $\mathrm{mL}$ ). Insoluble solids were removed by centrifugation and then red, block-shaped crystals of 2-Dy were grown from a layered THF-toluene solution ( $2 \mathrm{~mL}$ THF, $2 \mathrm{~mL}$ toluene) cooled to $-30{ }^{\circ} \mathrm{C}$ for $48 \mathrm{~h}$ (18.0 mg, 27\% yield). Anal. Calcd. For $\mathrm{C}_{86.5} \mathrm{H}_{106} \mathrm{BN}_{4} \mathrm{O}_{2} \mathrm{Dy}_{2}$ (2-Dy $\cdot 1.5$ toluene, observed in crystal structure): C, 66.19; H, 6.81; N, 3.57. Found: C, 66.30; H, 6.44; N, 3.52.

$\left[\left(\mathbf{D y C p}_{2}\right)_{2}\left(\boldsymbol{\mu - 5 , 5} \mathbf{5}^{\prime}-\mathbf{M e}_{2} \mathbf{b p y m}\right)\right]\left(\mathbf{B P h}_{4}\right)$ (3-Dy). Analogous to the synthesis of 1-Gd, $\left[\mathrm{Cp}_{2} \mathrm{Dy}\right]\left(\mathrm{BPh}_{4}\right)(53.3 \mathrm{mg}, 0.0707 \mathrm{mmol})$ was stirred in toluene $(4 \mathrm{~mL})$ with $\mathbf{M e}_{2} \mathbf{b p y m}(6.6 \mathrm{mg}$, $0.035 \mathrm{mmol}, 0.5$ equiv) and then $\mathrm{KC}_{8}(4.7 \mathrm{mg}, 0.035 \mathrm{mmol}, 0.5$ equiv) was added in THF (0.5 $\mathrm{mL}$ ). Insoluble solids were removed by centrifugation and then red, block-shaped crystals of 3-Dy were grown from a layered THF-toluene solution ( $2 \mathrm{~mL}$ THF, $2 \mathrm{~mL}$ toluene) cooled to $-30{ }^{\circ} \mathrm{C}$ for $48 \mathrm{~h}$ (33.2 mg, 69\% yield). Anal. Calcd. for $\mathrm{C}_{74} \mathrm{H}_{90} \mathrm{BN}_{4} \mathrm{Dy} 2: \mathrm{C}, 64.81 ; \mathrm{H}, 6.62 ; \mathrm{N}, 4.09$. Found: $\mathrm{C}$, 64.46; H, 6.34; N, 3.78.

$\left[\left(\mathbf{D y C p}^{*}\right)_{2}\left(\boldsymbol{\mu}-\mathbf{5 , 5} \mathbf{5}^{\prime}-\mathbf{F}_{2} \mathbf{b p y m}\right)\right]\left(\mathbf{B P h}_{4}\right)$ (4-Dy). Analogous to the synthesis of 4-Gd, $\left[\mathrm{Cp}_{2}{ }_{2} \mathrm{Dy}\right]\left(\mathrm{BPh}_{4}\right)(77.5 \mathrm{mg}, 0.103 \mathrm{mmol})$ was stirred in toluene $(4 \mathrm{~mL})$ with $\mathbf{F}_{2} \mathbf{b p y m}(10.0 \mathrm{mg}$, $0.0515 \mathrm{mmol}, 0.5$ equiv) and then reduced with $\mathrm{KC}_{8}(6.9 \mathrm{mg}, 0.052 \mathrm{mmol}, 0.5$ equiv) for $24 \mathrm{~h}$. The reaction was concentrated under reduced pressure, dissolved in DFB, and filtered through diatomaceous earth. Red, block-shaped crystals of 4-Dy were grown from a layered DFB-hexanes solution ( $2 \mathrm{~mL}$ DFB, $2 \mathrm{~mL}$ hexanes) stored at $25^{\circ} \mathrm{C}$ for $48 \mathrm{~h}(36.1 \mathrm{mg}, 51 \%$ yield). Anal. Calcd. For $\mathrm{C}_{76} \mathrm{H}_{96} \mathrm{BN}_{6} \mathrm{Dy} 2: \mathrm{C}, 62.70 ; \mathrm{H}, 6.14 ; \mathrm{N}, 4.06$. Found: $\mathrm{C}, 62.90 ; \mathrm{H}, 6.47 ; \mathrm{N}, 3.68$.

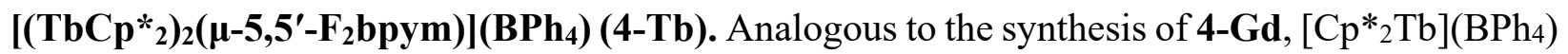
(77.1 mg, $0.103 \mathrm{mmol})$ was stirred in toluene $(10 \mathrm{~mL})$ with $\mathbf{F}_{2} \mathbf{b p y m}(10.0 \mathrm{mg}, 0.0515 \mathrm{mmol}, 0.5$ equiv) and then reduced with $\mathrm{KC}_{8}(7.0 \mathrm{mg}, 0.052 \mathrm{mmol}, 0.5$ equiv) for $24 \mathrm{~h}$. The solvent was removed under reduced pressure, and the reaction mixture was dissolved in DFB, and filtered through diatomaceous earth to remove insoluble solids. Red, block-shaped crystals of 4-Tb were grown from a layered DFB-hexanes solution (2mL DFB, $2.5 \mathrm{~mL}$ hexanes) stored at $-30^{\circ} \mathrm{C}$ for 24 
h (20 mg, 14\% yield). Anal. Calcd. For $\mathrm{C}_{76} \mathrm{H}_{84} \mathrm{BF}_{2} \mathrm{~N}_{4} \mathrm{~Tb}_{2}$ : C, 63.02; H, 6.17; N, 4.08. Found: C, $62.92 ; \mathrm{H}, 5.98 ; \mathrm{N}, 4.02$.

\section{Section S2. NMR spectroscopy}
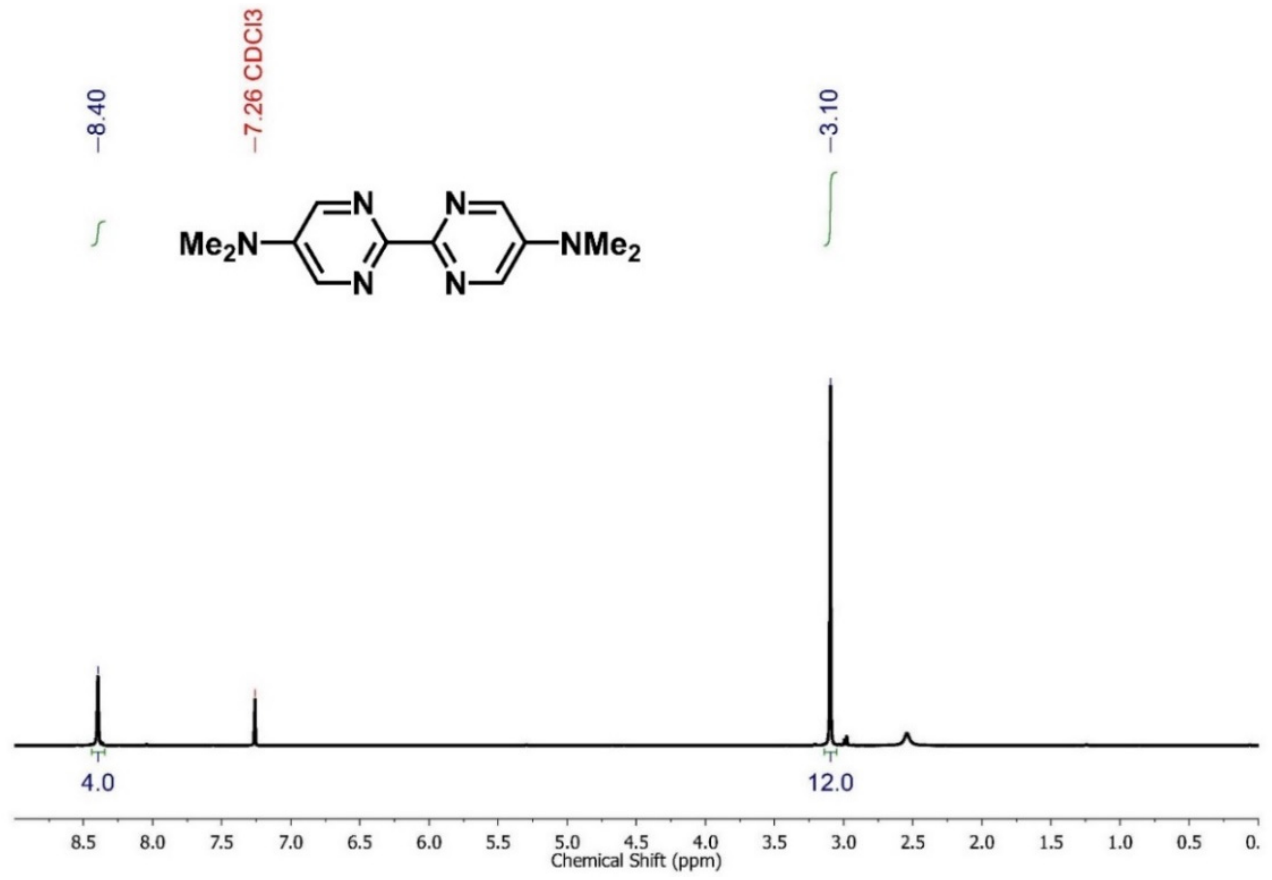

Figure S1. ${ }^{1} \mathrm{H}-\mathrm{NMR}$ spectrum of $\left(\mathbf{N M e}_{2}\right)_{2} \mathbf{b p y m}$ in $\mathrm{CDCl}_{3}$.

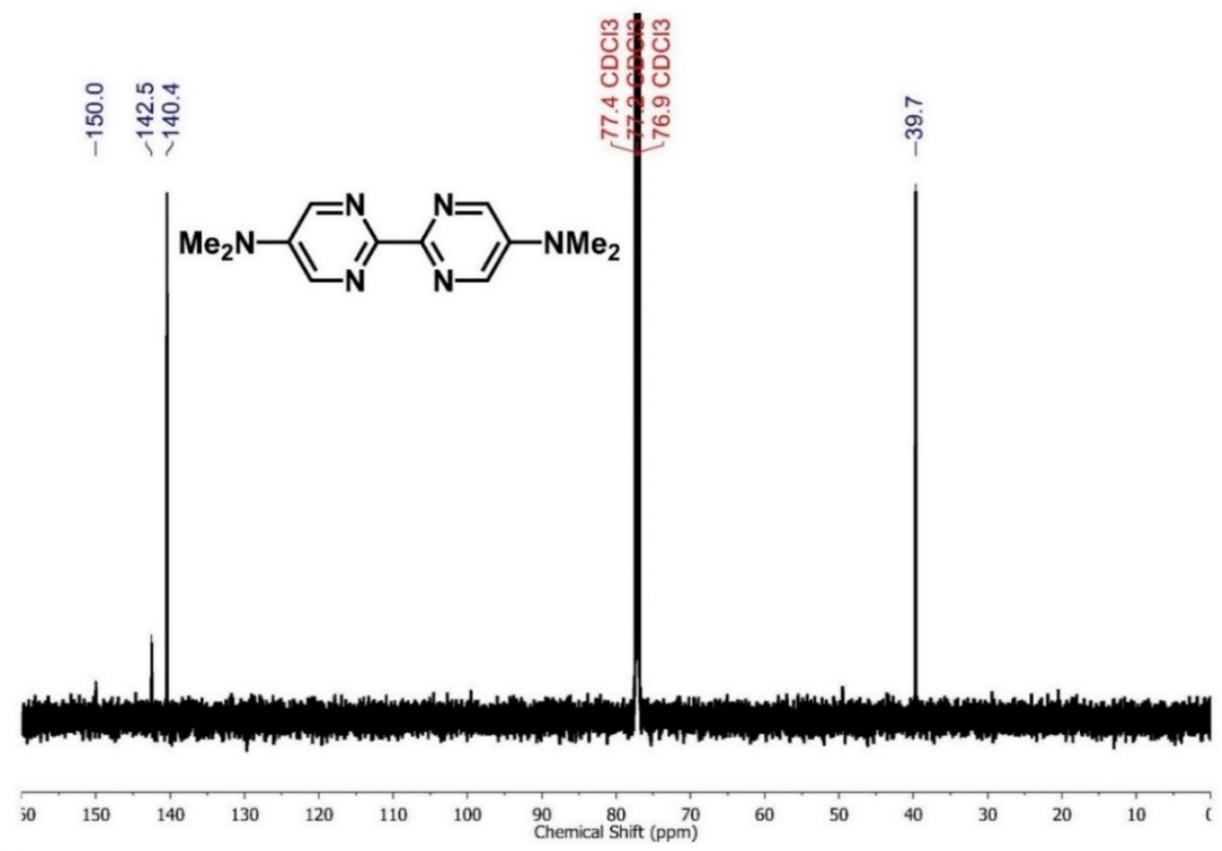

Figure S2. ${ }^{13} \mathrm{C}-\mathrm{NMR}$ spectrum of $\left(\mathbf{N M e}_{2}\right)_{2} \mathbf{b p y m}$ in $\mathrm{CDCl}_{3}$. 

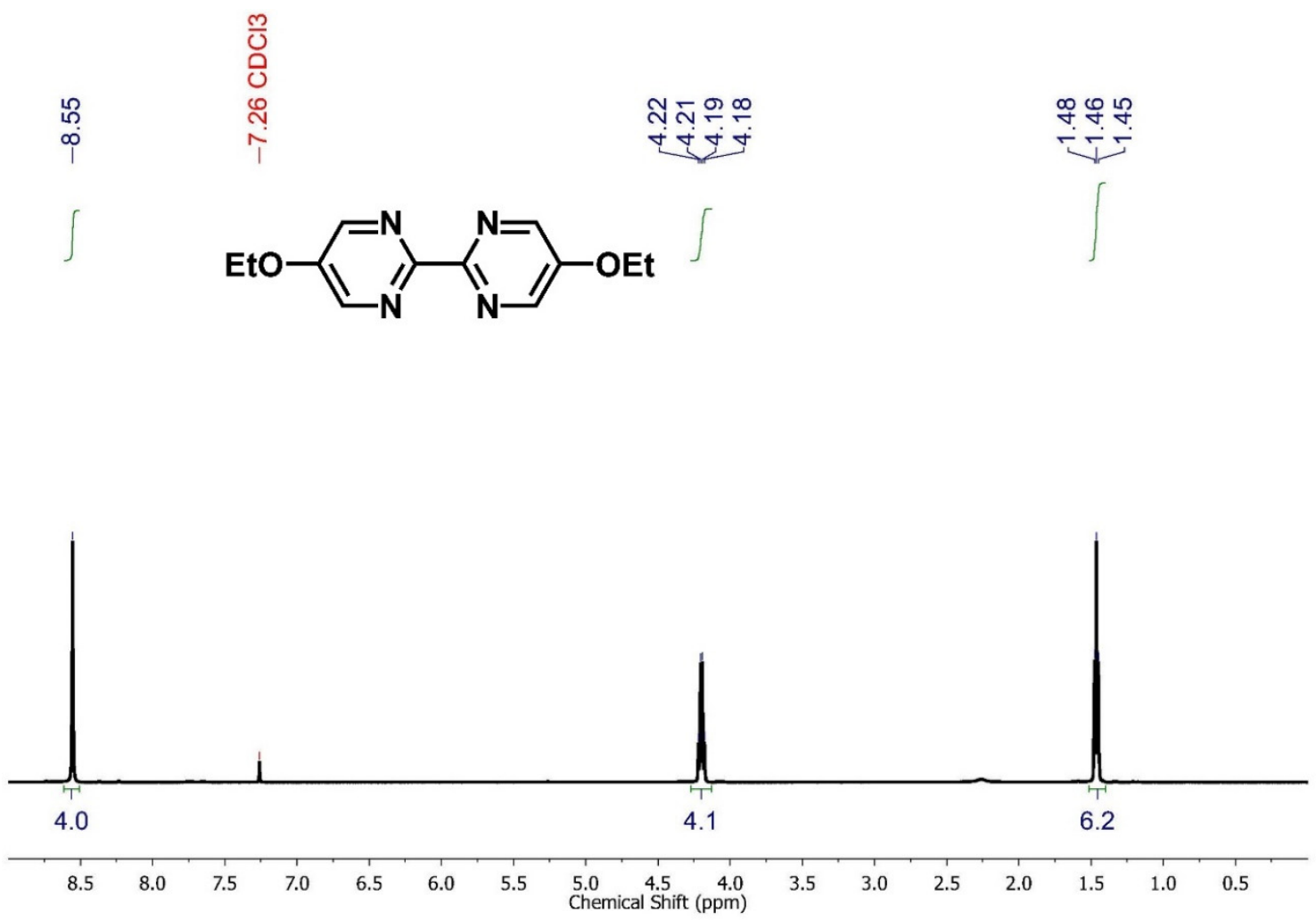

Figure S3. ${ }^{1} \mathrm{H}-\mathrm{NMR}$ spectrum of $(\mathbf{O E t})_{2} \mathbf{b p y m}$ in $\mathrm{CDCl}_{3}$.

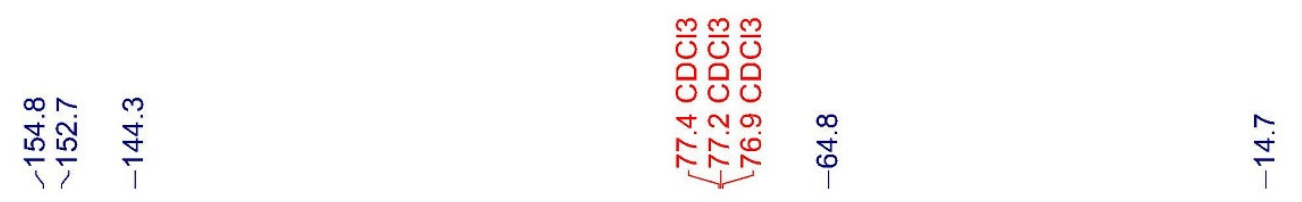<smiles>CCOc1cnc(-c2ncc(OCC)cn2)nc1</smiles>

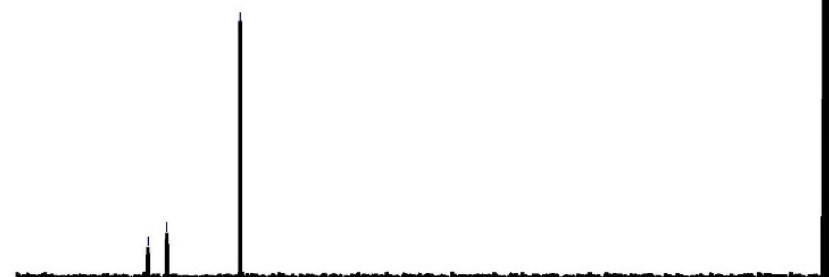

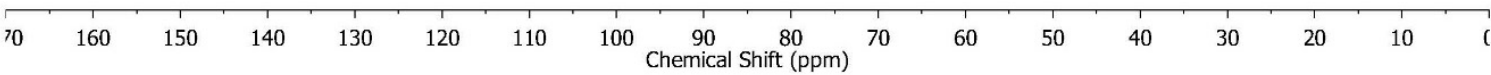

Figure S4. ${ }^{13} \mathrm{C}-\mathrm{NMR}$ spectrum of (OEt) $)_{2}$ bpym in $\mathrm{CDCl}_{3}$. 

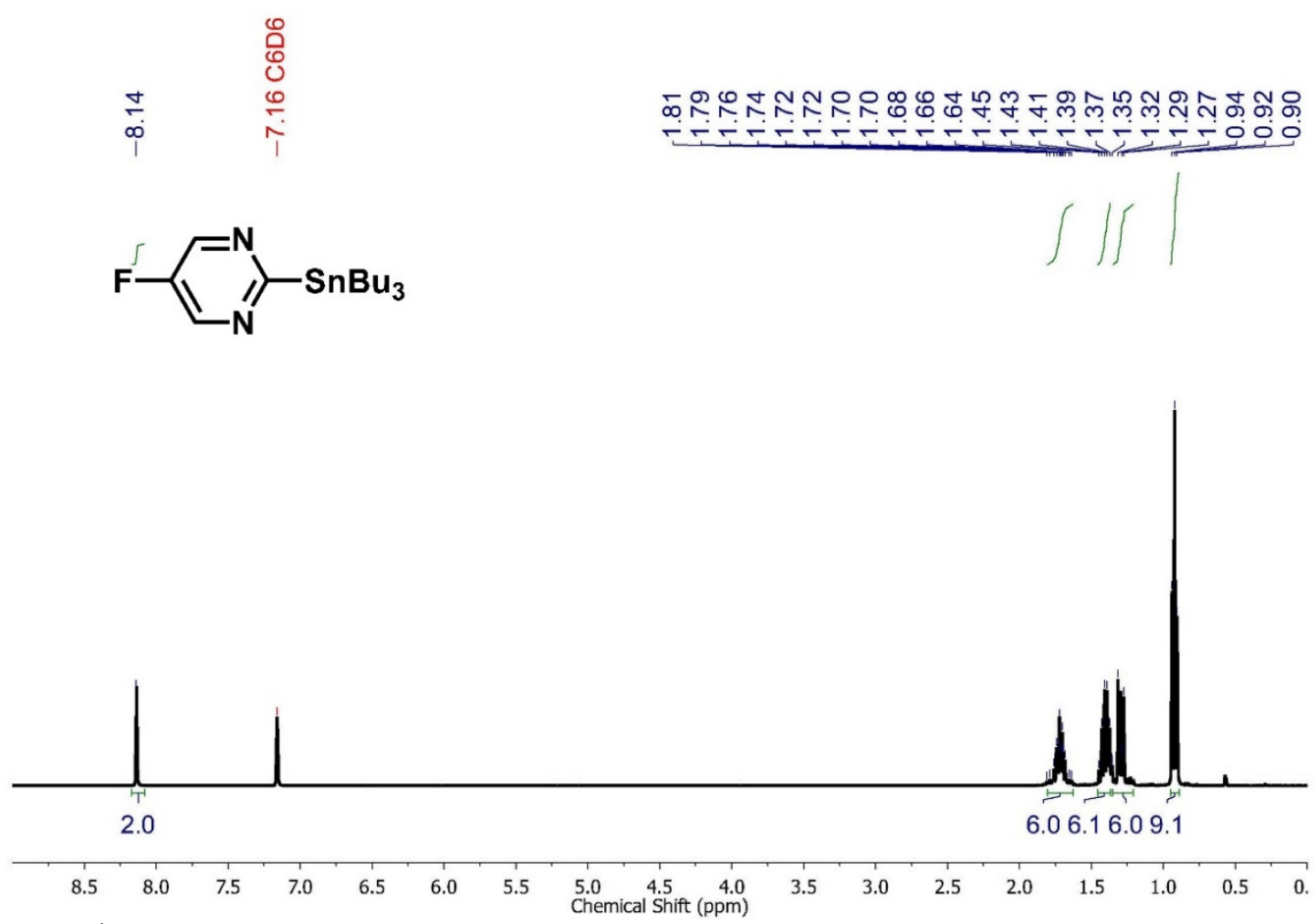

Figure S5. ${ }^{1} \mathrm{H}-\mathrm{NMR}$ spectrum of 5-fluoro-2-tributylstannylpyrimidine in $\mathrm{C}_{6} \mathrm{D}_{6}$.
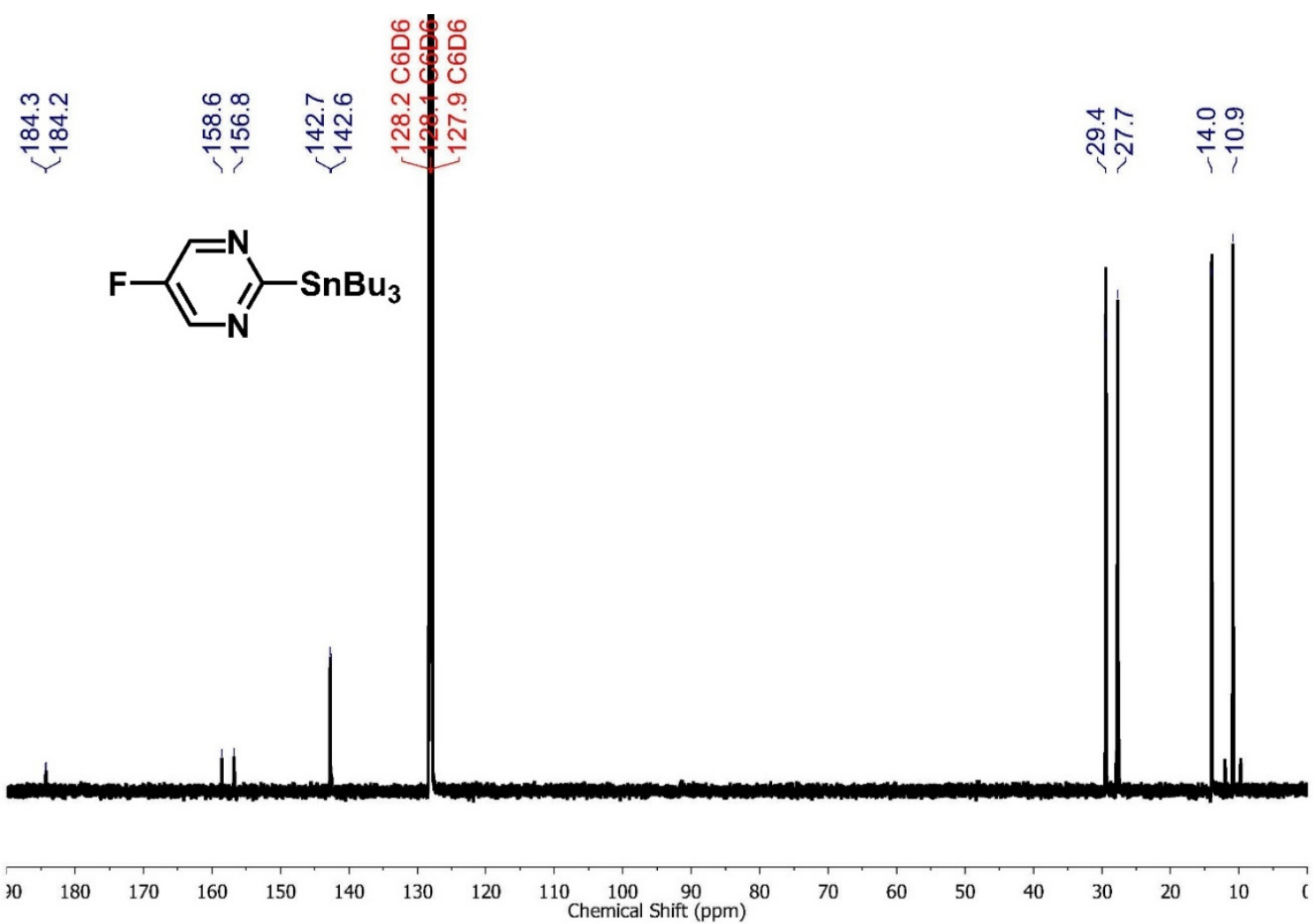

Figure S6. ${ }^{13} \mathrm{C}$-NMR spectrum of 5-fluoro-2-tributylstannylpyrimidine in $\mathrm{C}_{6} \mathrm{D}_{6}$. 
<smiles>CCCCSc1ncc(F)cn1</smiles>

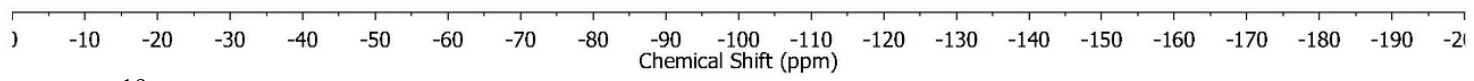

Figure S7. ${ }^{19}$ F-NMR spectrum of 5-fluoro-2-tributylstannylpyrimidine in $\mathrm{C}_{6} \mathrm{D}_{6}$.
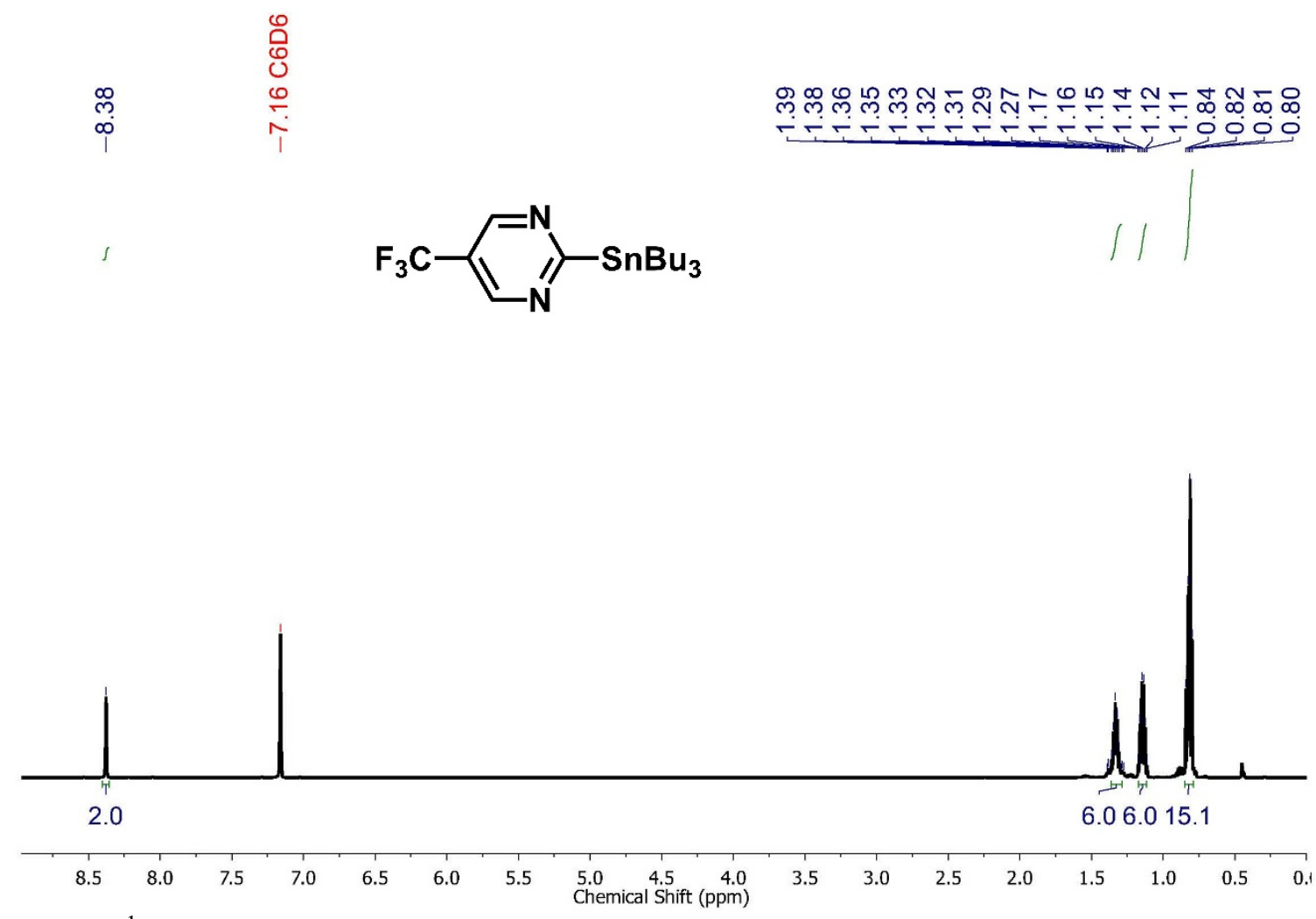

Figure S8. ${ }^{1} \mathrm{H}-\mathrm{NMR}$ spectrum of 5-trifluoromethyl-2-tributylstannylpyrimidine in $\mathrm{C}_{6} \mathrm{D}_{6}$. 


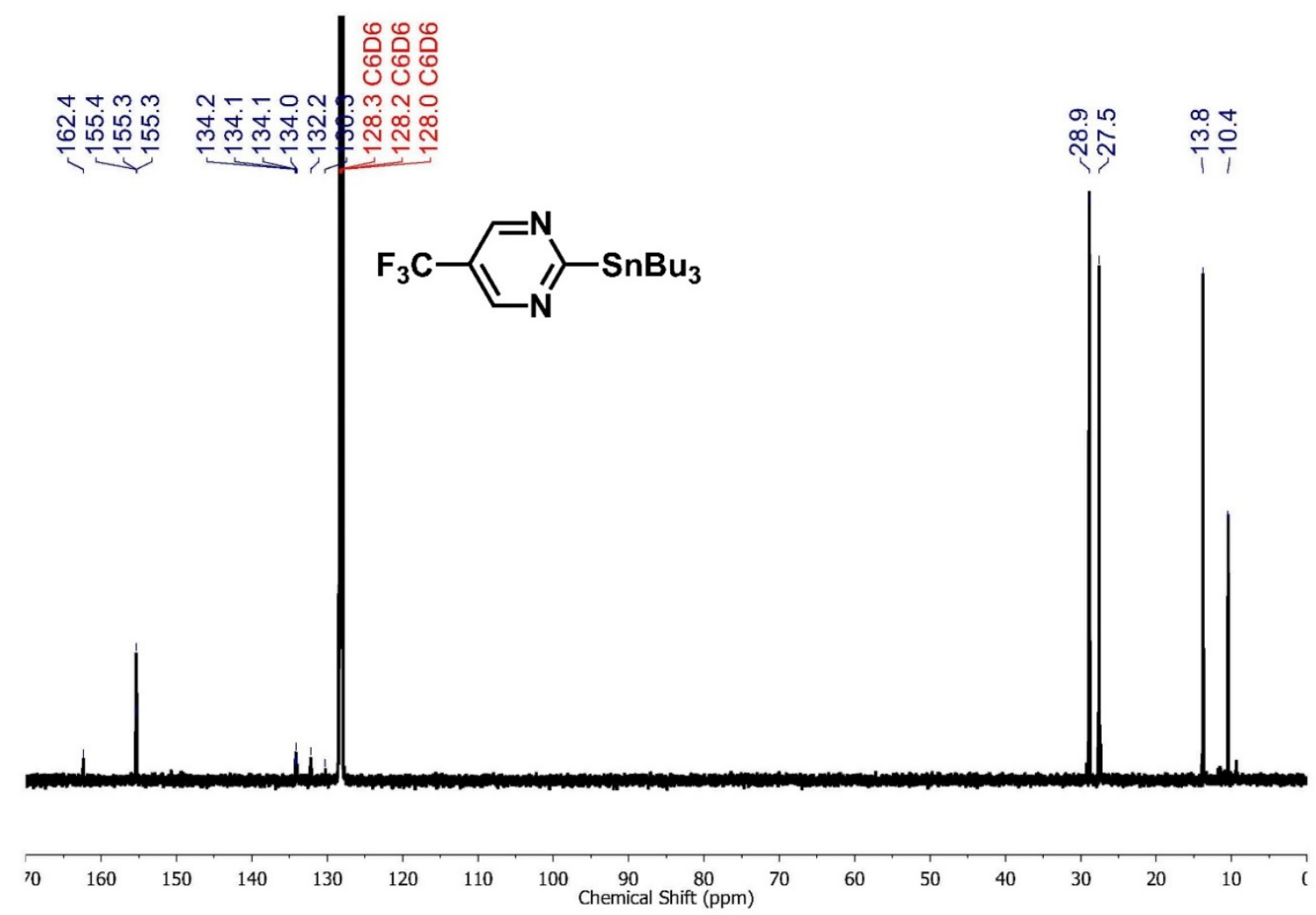

Figure S9. ${ }^{13} \mathrm{C}$-NMR spectrum of 5-trifluoromethyl -2-tributylstannylpyrimidine in $\mathrm{C}_{6} \mathrm{D}_{6}$.

ণ্<smiles>CCCCc1ncc(C(F)(F)F)cn1</smiles>

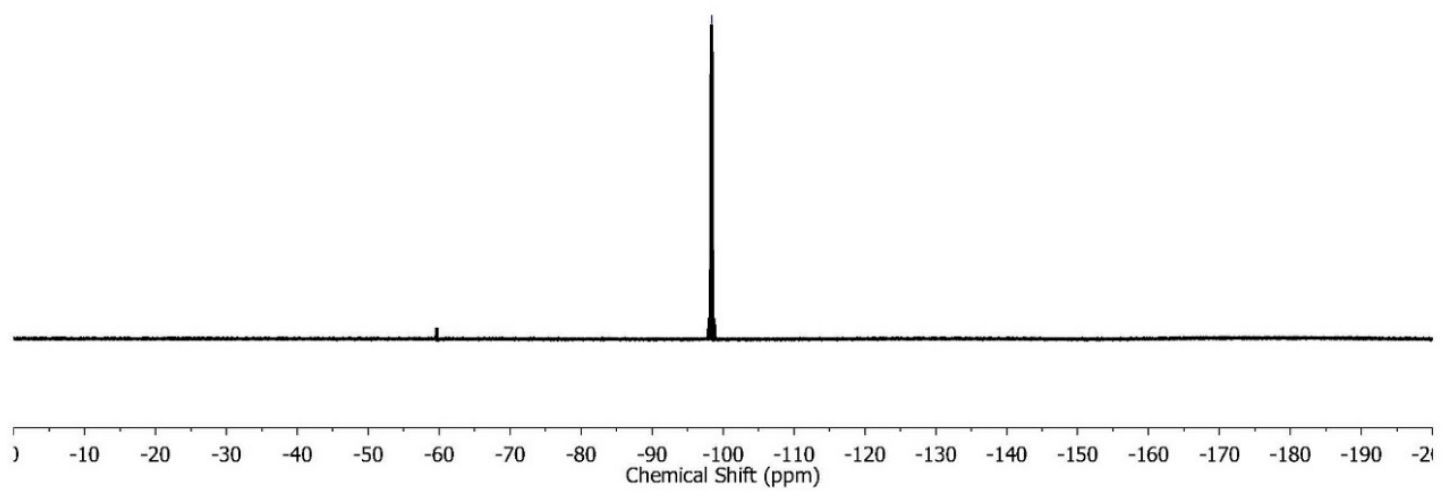

Figure S10. ${ }^{19}$ F-NMR spectrum of 5-trifluoromethyl -2-tributylstannylpyrimidine in $\mathrm{C}_{6} \mathrm{D}_{6}$. 

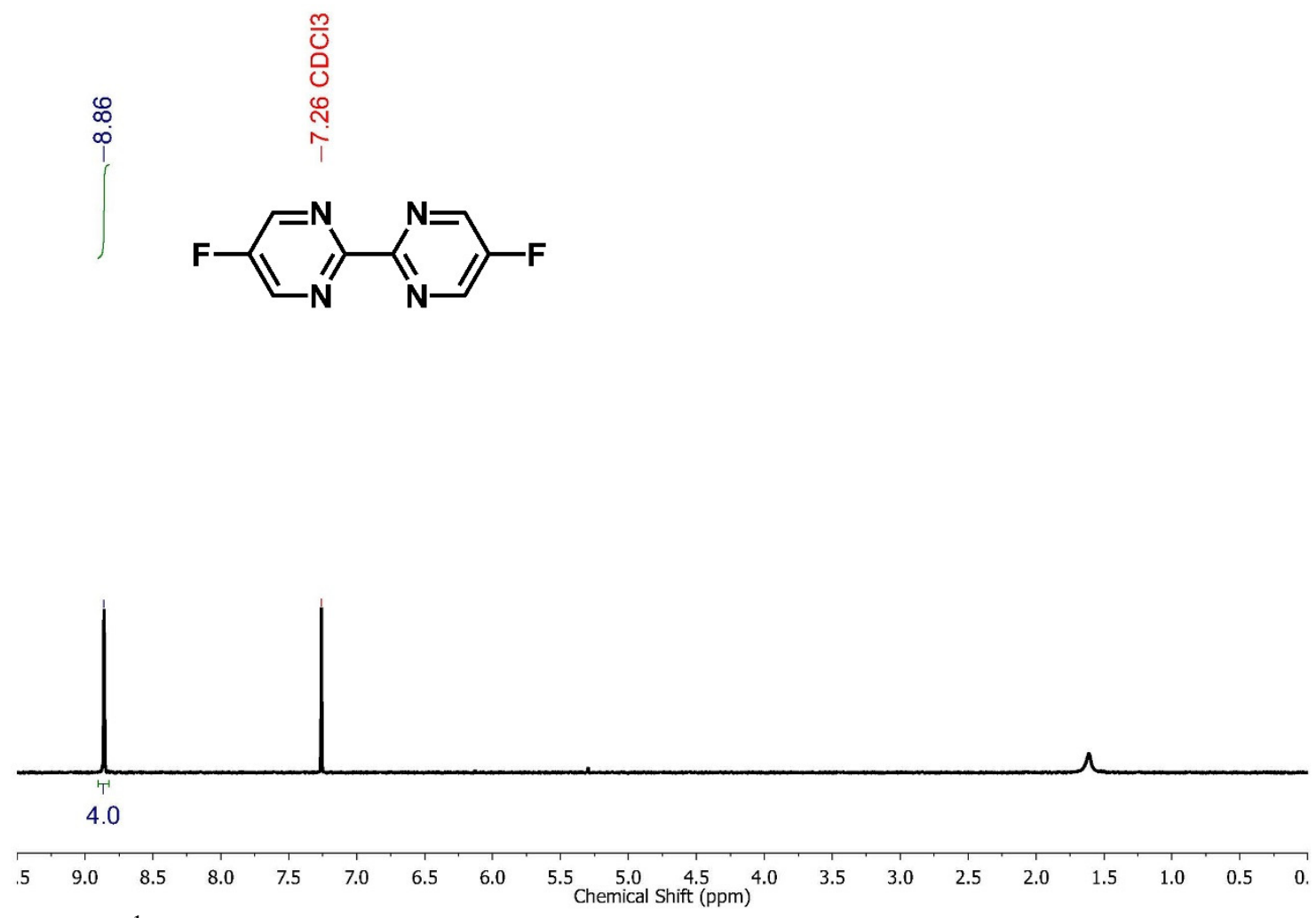

Figure S11. ${ }^{1} \mathrm{H}-\mathrm{NMR}$ spectrum of $\mathbf{F}_{2} \mathbf{b p y m}$ in $\mathrm{CDCl}_{3}$.
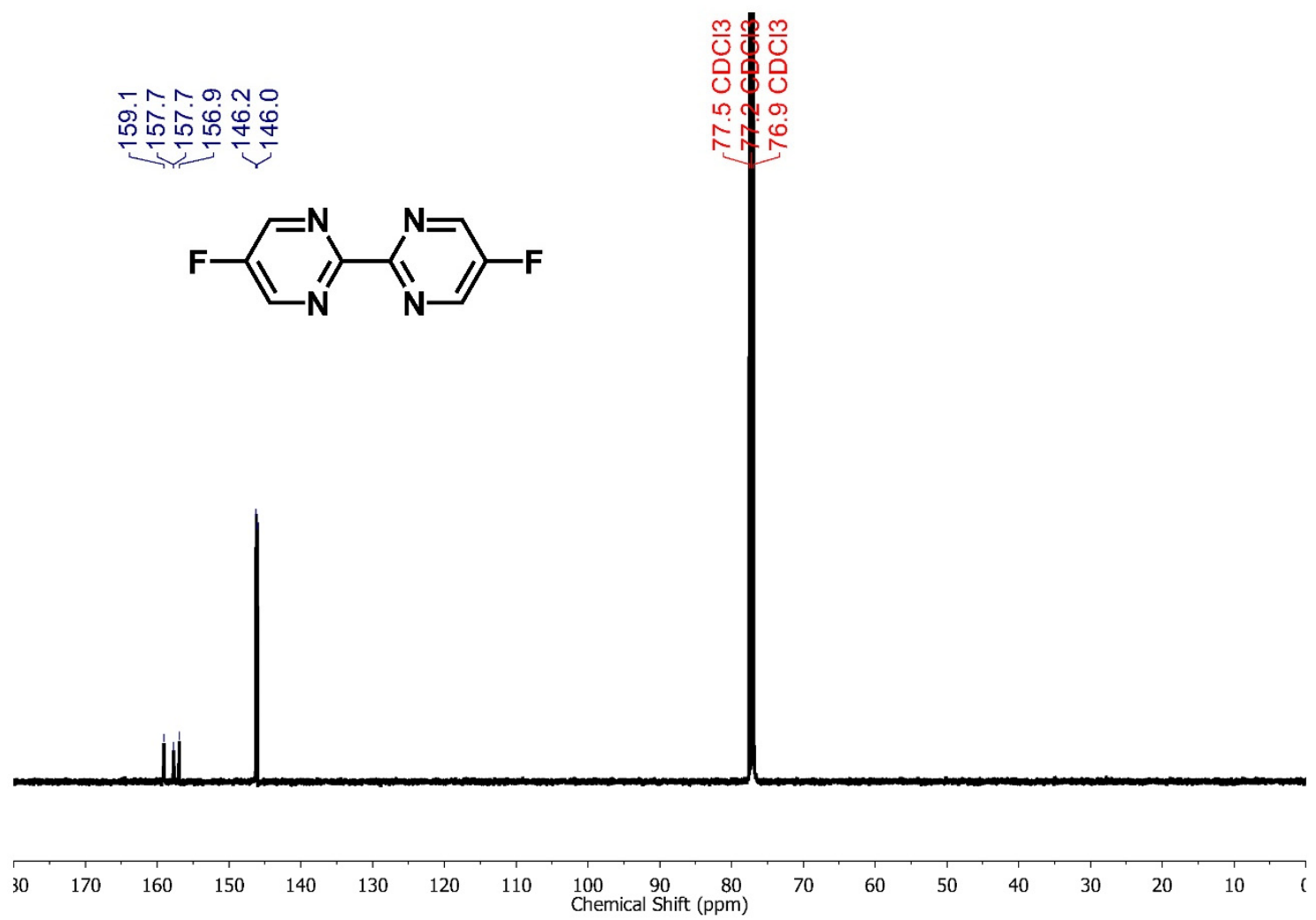

Figure S12. ${ }^{13} \mathrm{C}-\mathrm{NMR}$ spectrum of $\mathbf{F}_{2} \mathbf{b p y m}$ in $\mathrm{CDCl}_{3}$. 
<smiles>Fc1cnc(-c2ncc(F)cn2)nc1</smiles>

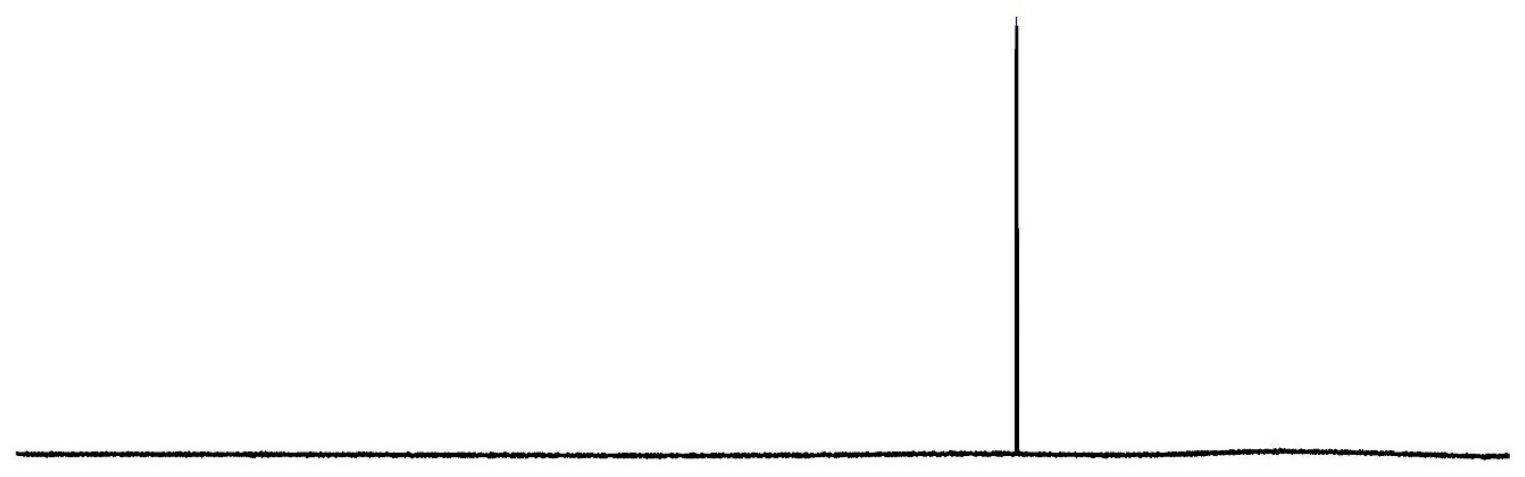

\begin{tabular}{rlllllllllllllllllllll}
\hline & -10 & -20 & -30 & -40 & -50 & -60 & -70 & -80 & -90 & -100 & -110 & -120 & -130 & -140 & -150 & -160 & -170 & -180 & -190 & -21 \\
\hline
\end{tabular}

Figure S13. ${ }^{19} \mathrm{~F}-\mathrm{NMR}$ spectrum of $\mathbf{F}_{2} \mathbf{b p y m}$ in $\mathrm{CDCl}_{3}$.
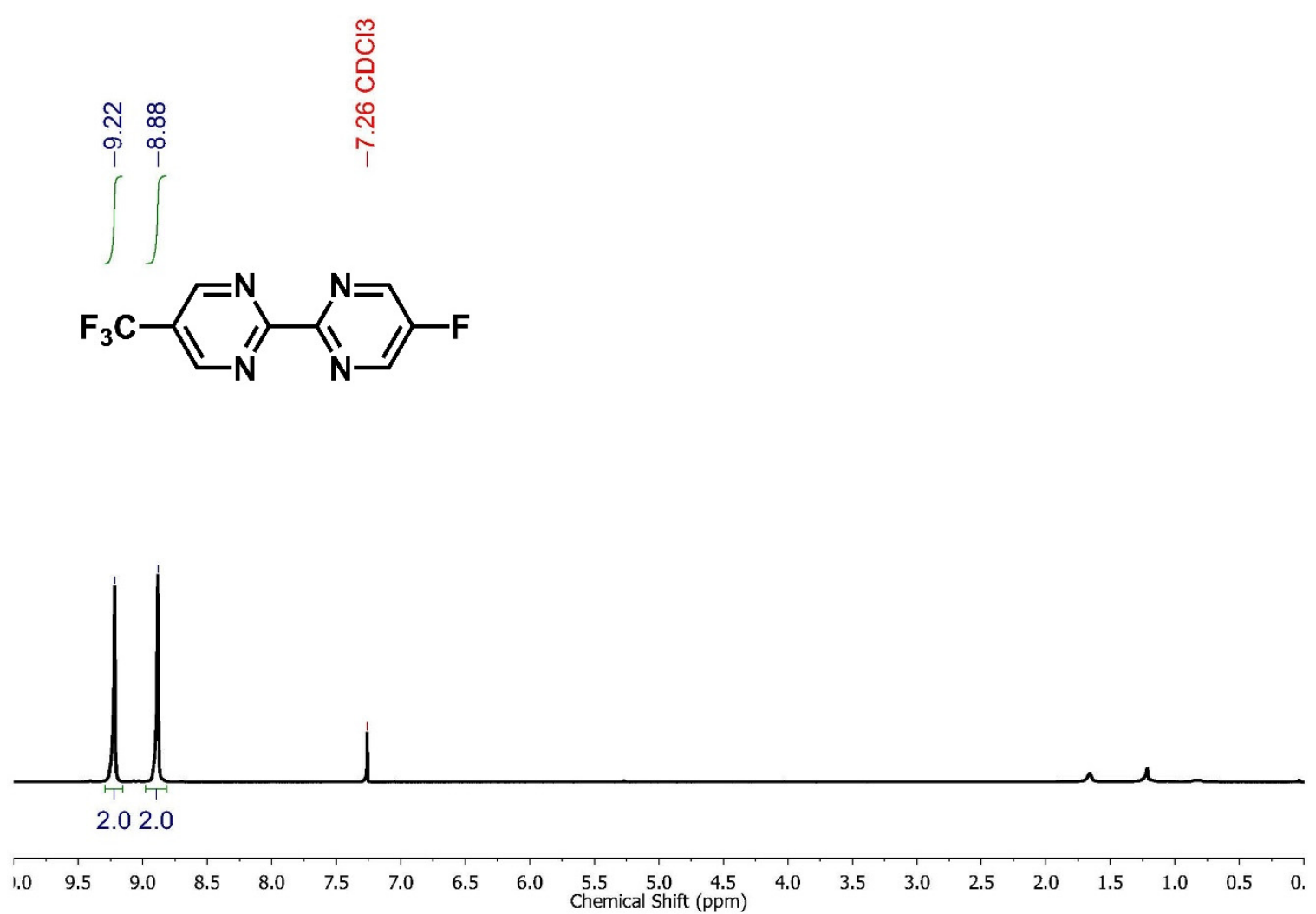

Figure S14. ${ }^{1} \mathrm{H}-\mathrm{NMR}$ spectrum of $\mathbf{F}\left(\mathbf{C F}_{3}\right)$ bpym in $\mathrm{CDCl}_{3}$. 

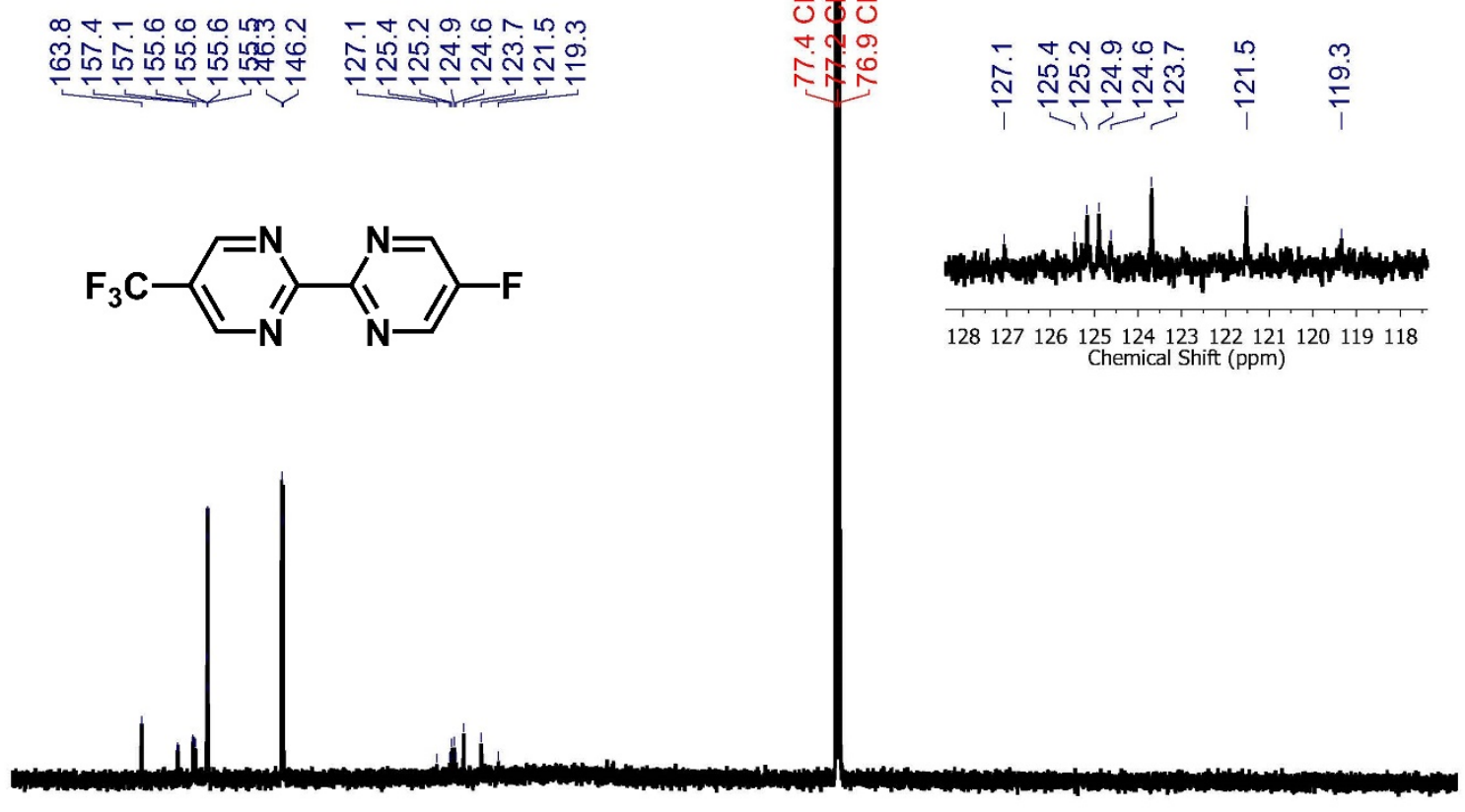

\begin{tabular}{|c|c|c|c|c|c|c|c|c|c|c|c|c|c|c|c|c|}
\hline 30 & 170 & 160 & 150 & 140 & 130 & 120 & 110 & $\begin{array}{l}100 \\
\text { Chemi }\end{array}$ & $\begin{array}{cc}90 & 80 \\
\text { Shift } & (\mathrm{ppm})\end{array}$ & 70 & 60 & 50 & 40 & 30 & 20 & 10 \\
\hline
\end{tabular}

Figure S15. ${ }^{13} \mathrm{C}-\mathrm{NMR}$ spectrum of $\mathbf{F}\left(\mathbf{C F}_{3}\right)$ bpym in $\mathrm{CDCl}_{3}$.

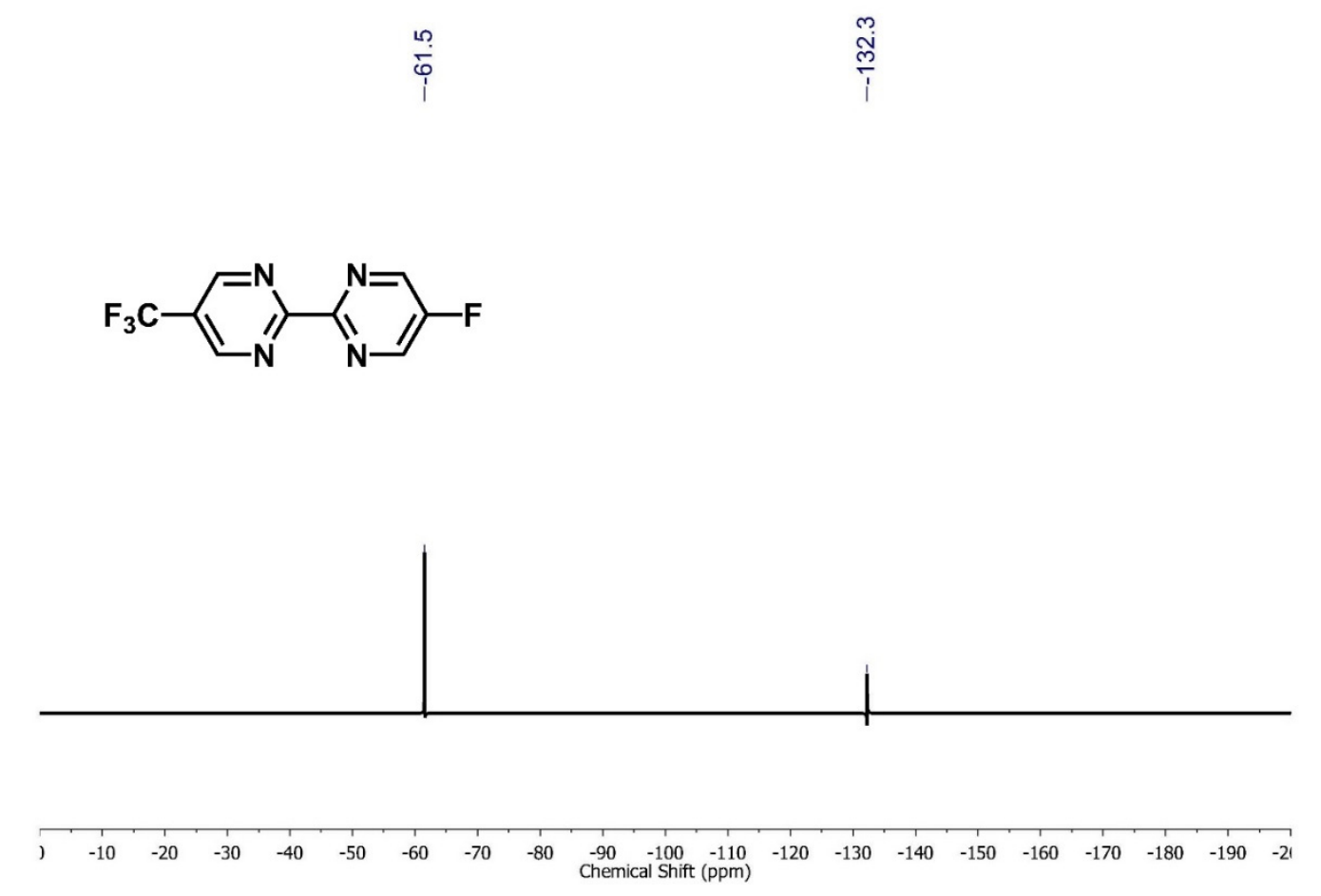

Figure S16. ${ }^{19}$ F-NMR spectrum of $\mathbf{F}\left(\mathbf{C F}_{3}\right)$ bpym in $\mathrm{CDCl}_{3}$. 


\section{Section S3. Infrared spectroscopy}

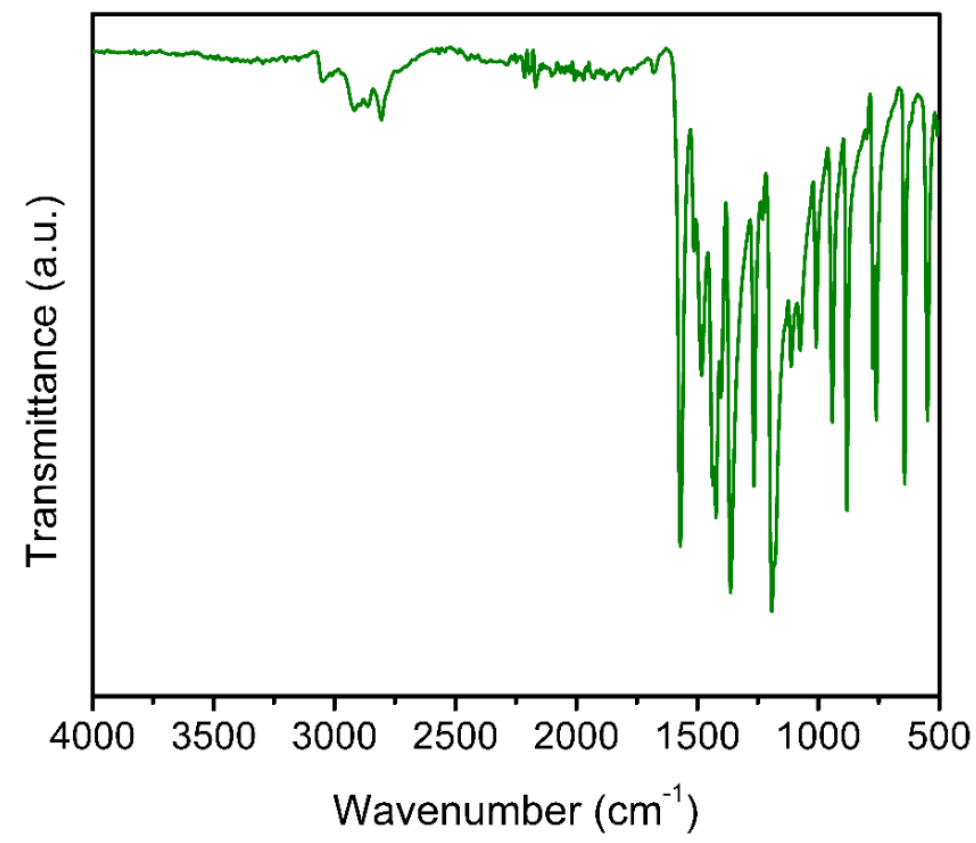

Figure S17. IR spectrum of $\left(\mathrm{NMe}_{2}\right)_{2} \mathbf{b p y m}$.

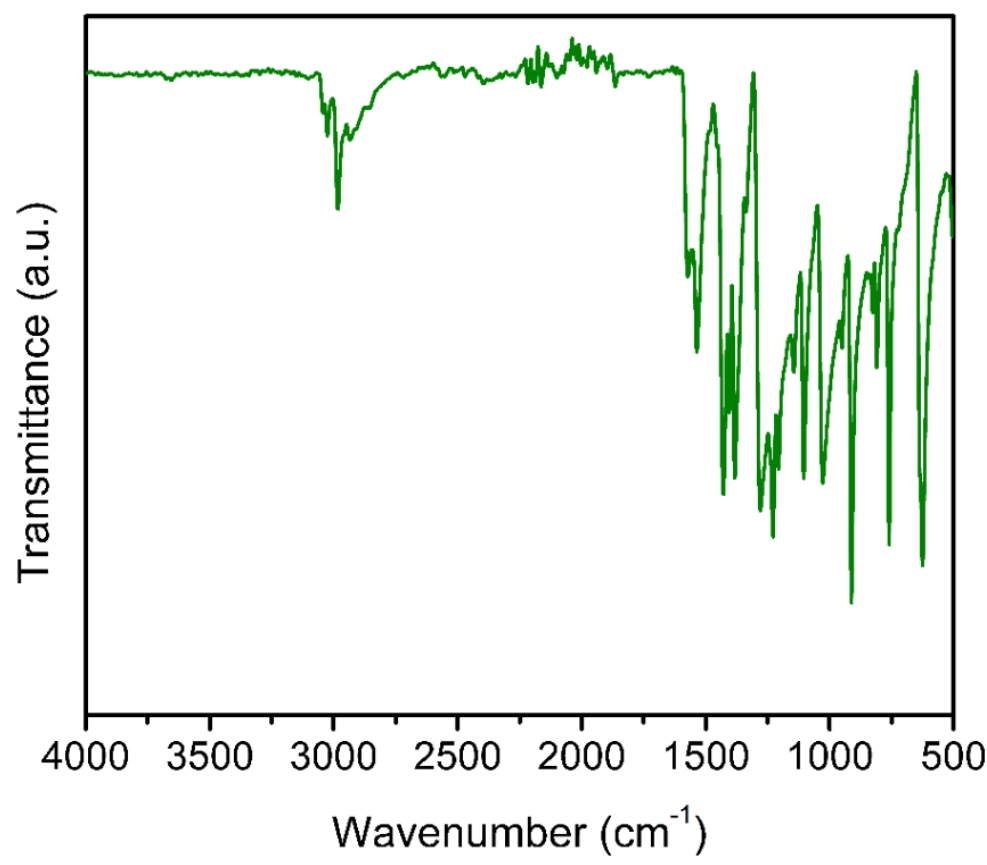

Figure S18. IR spectrum of $(\mathrm{OEt})_{2}$ bpym. 


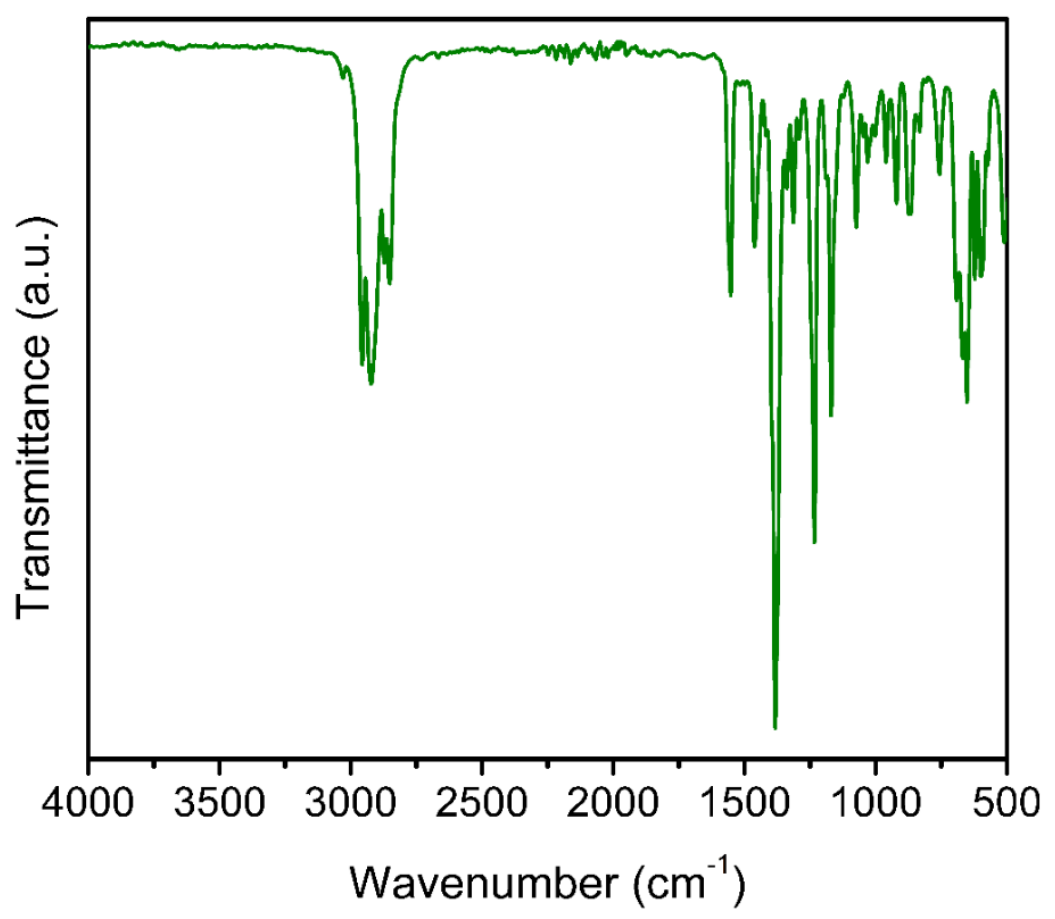

Figure S19. IR spectrum of 5-fluoro-2-tributylstannylpyrimidine.

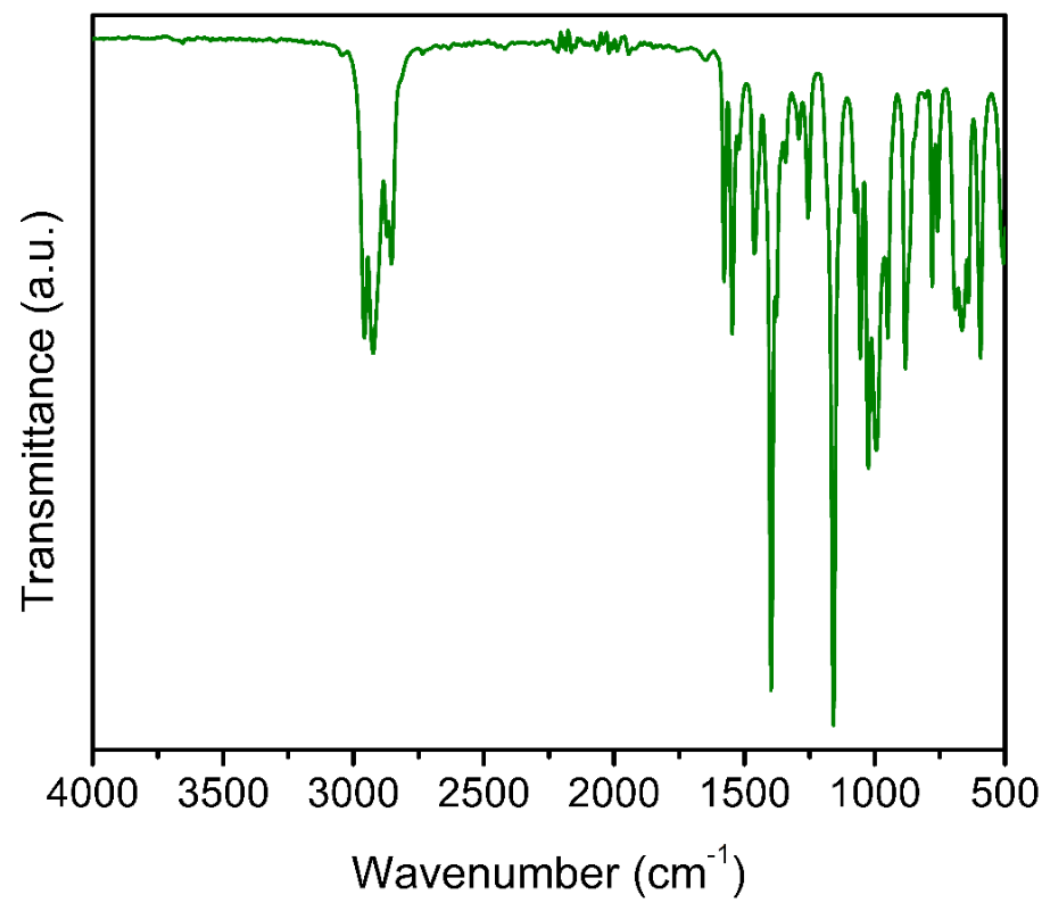

Figure S20. IR spectrum of 5-trifluoromethyl-2-tributylstannylpyrimidine. 


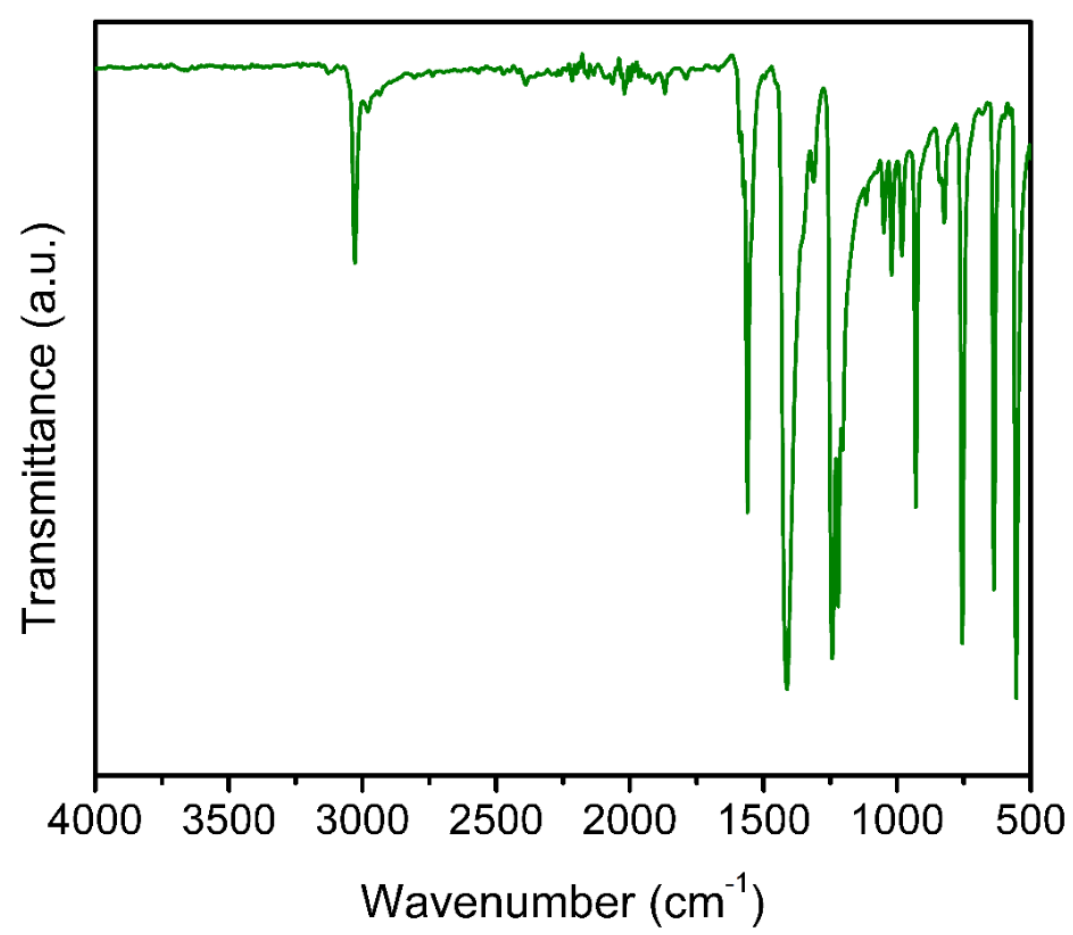

Figure S21. IR spectrum of $\mathbf{F}_{2} \mathbf{b p y m}$.

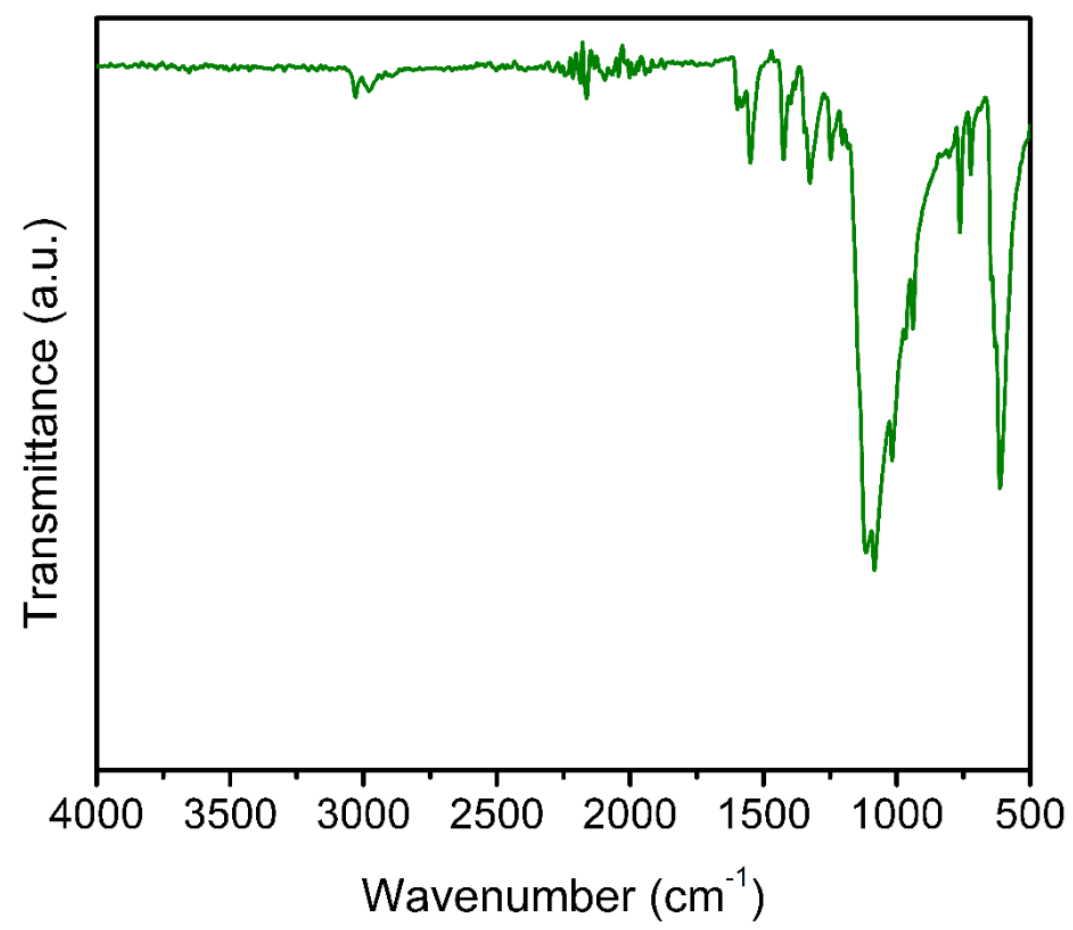

Figure S22. IR spectrum of $\mathbf{F}\left(\mathrm{CF}_{3}\right)$ bpym. 


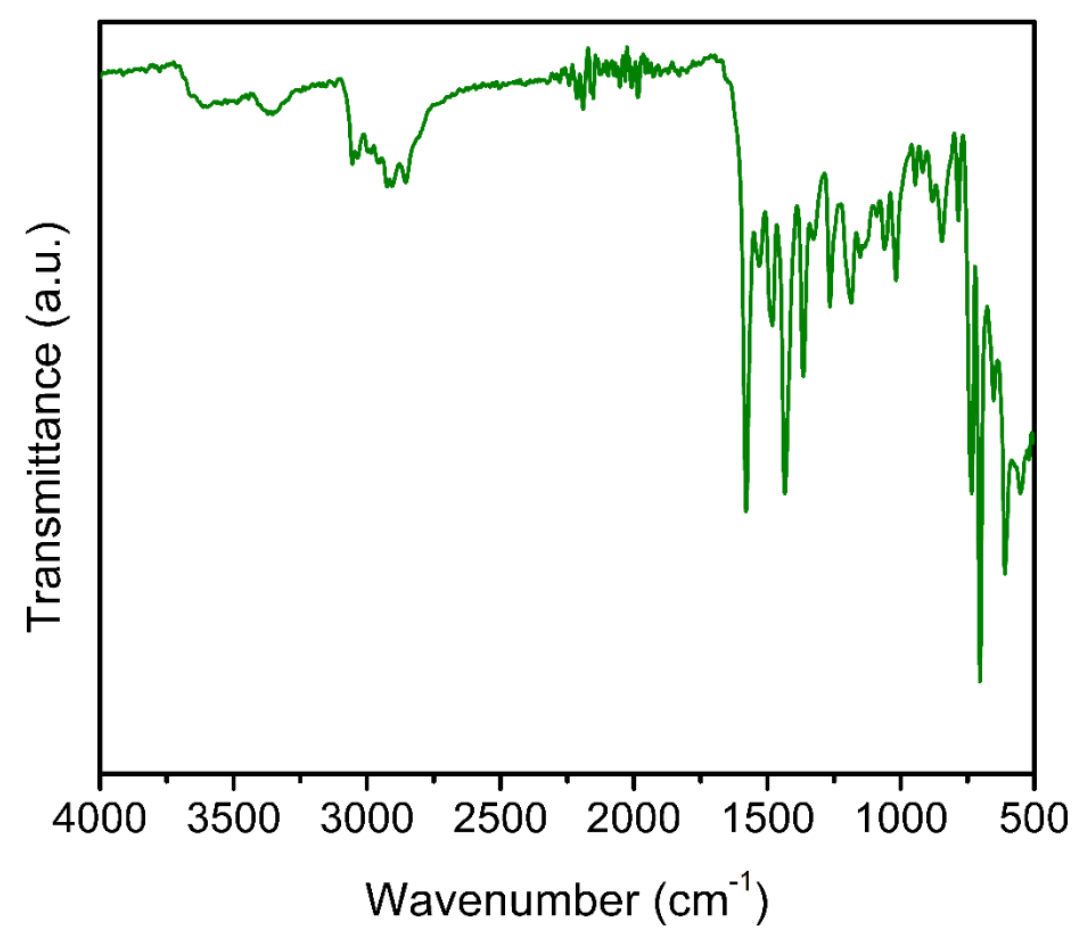

Figure S23. IR spectrum of 1-Gd.

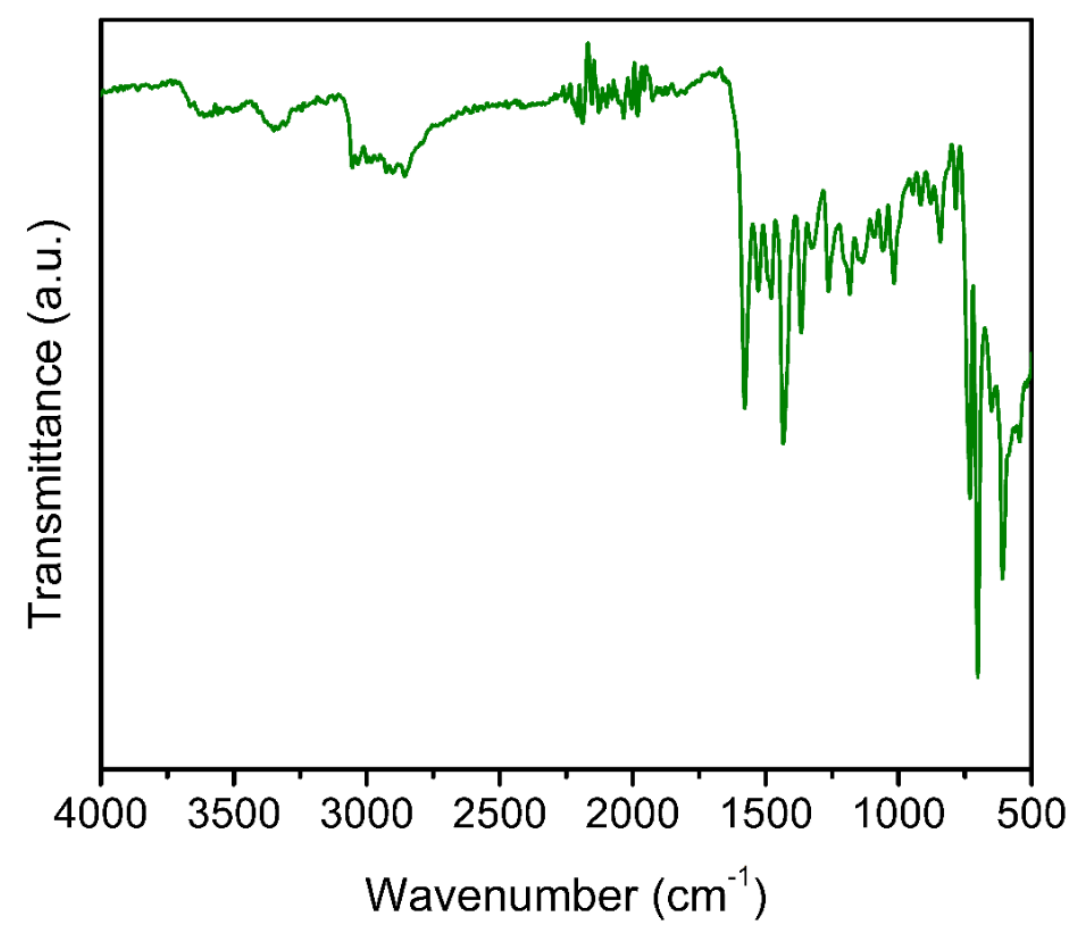

Figure S24. IR spectrum of 1-Dy. 


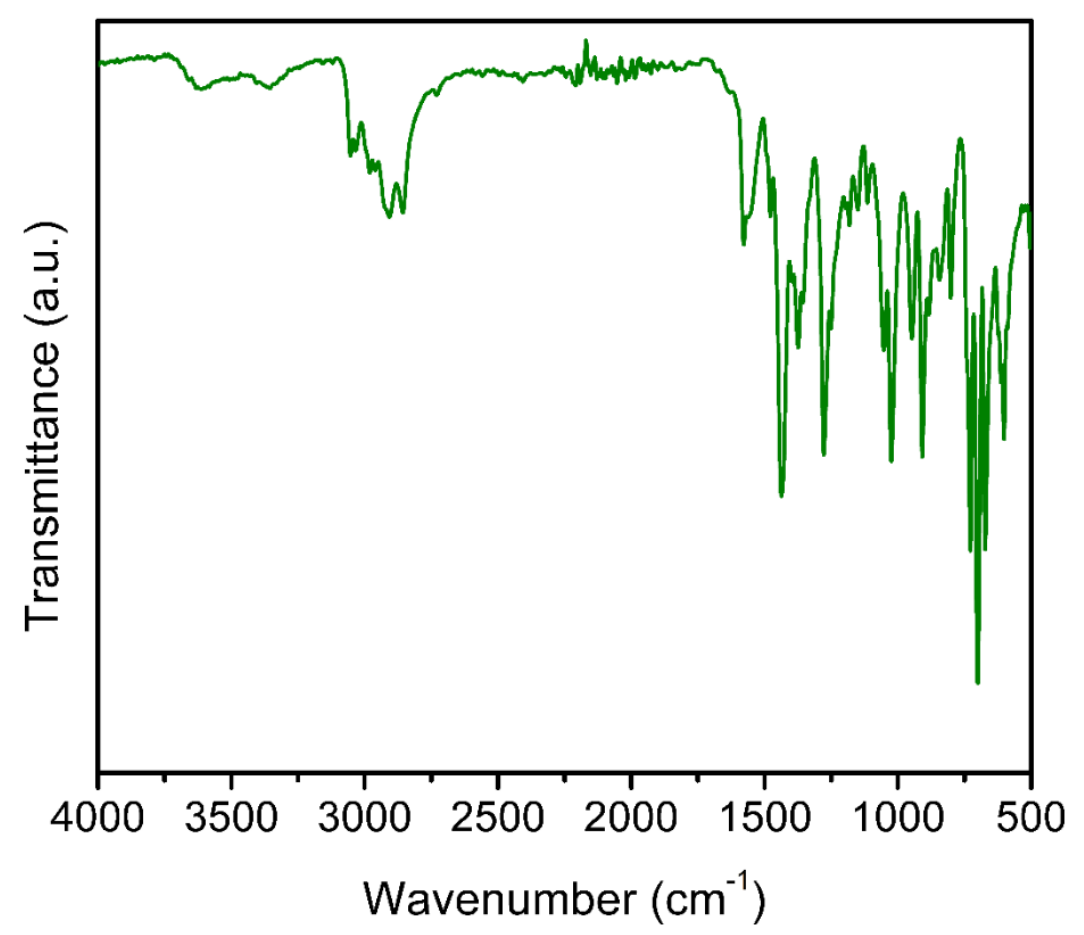

Figure S25. IR spectrum of 2-Gd.

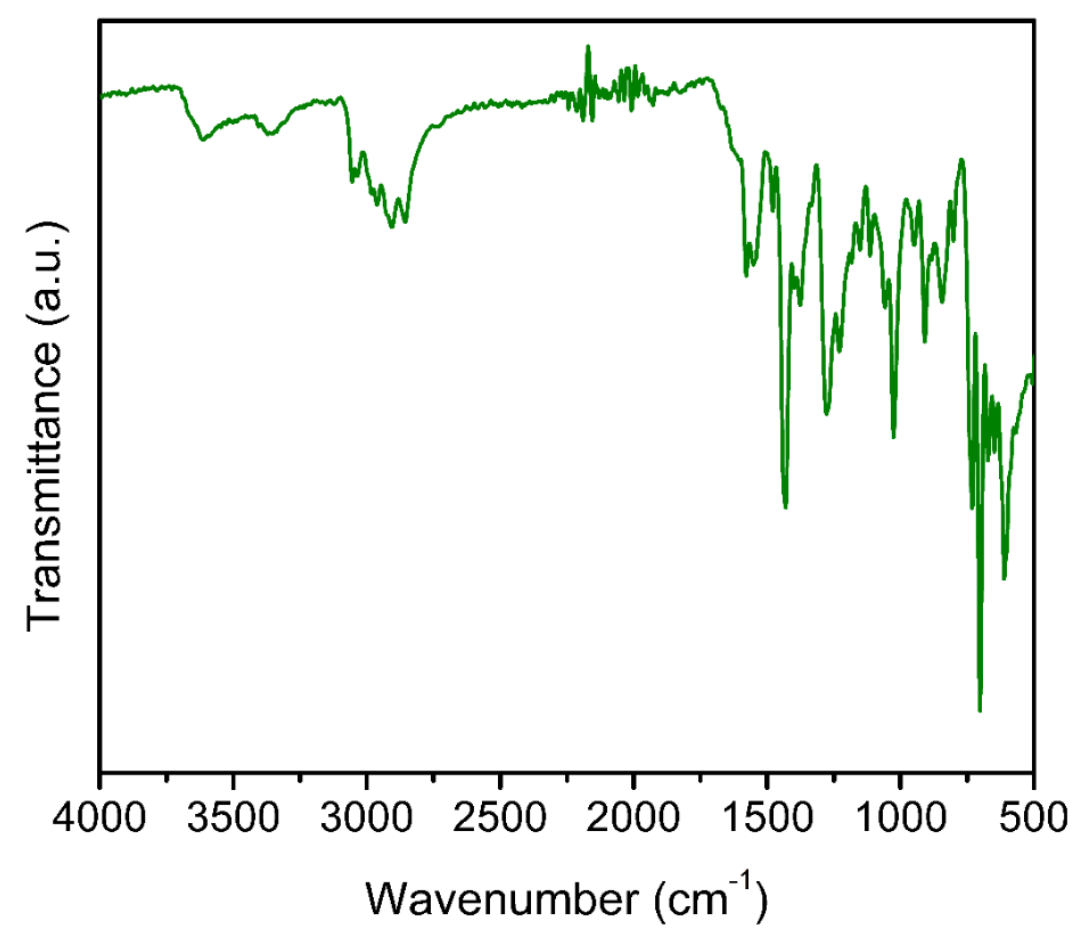

Figure S26. IR spectrum of 2-Dy. 


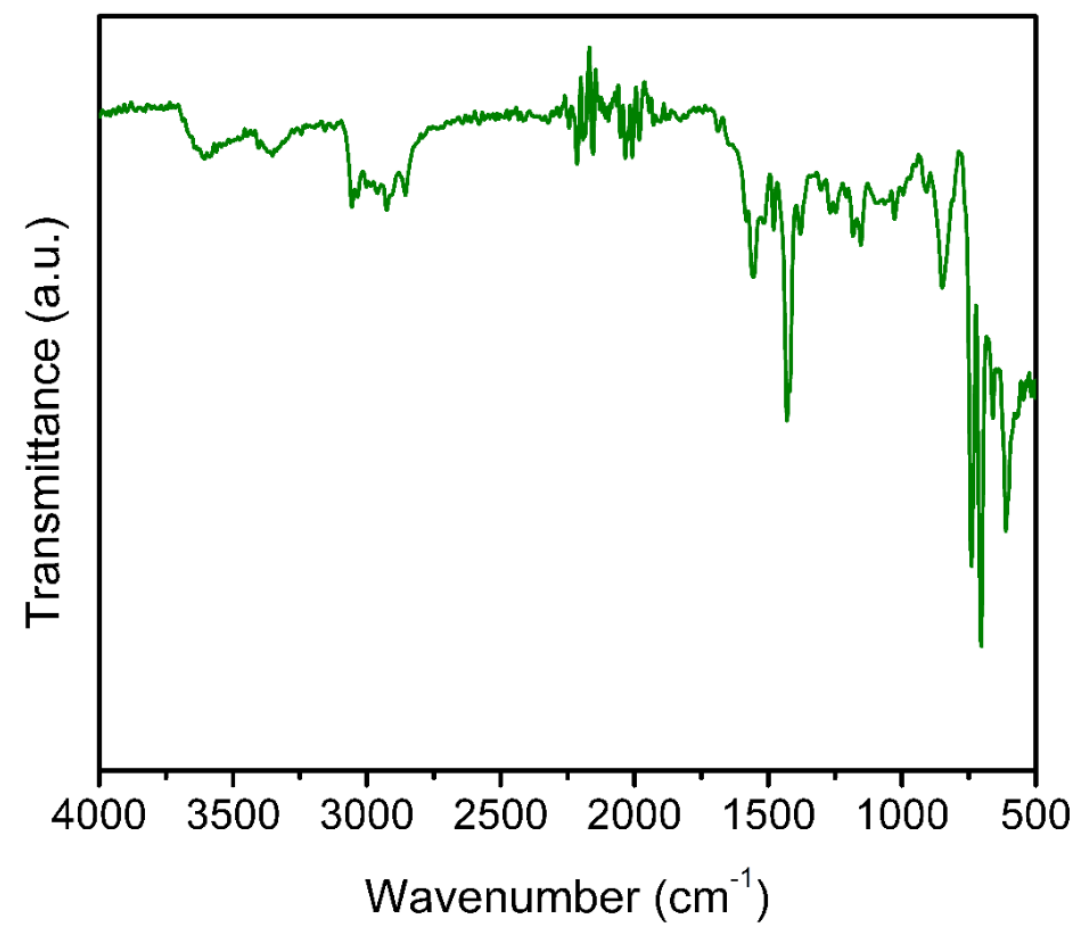

Figure S27. IR spectrum of 3-Gd.

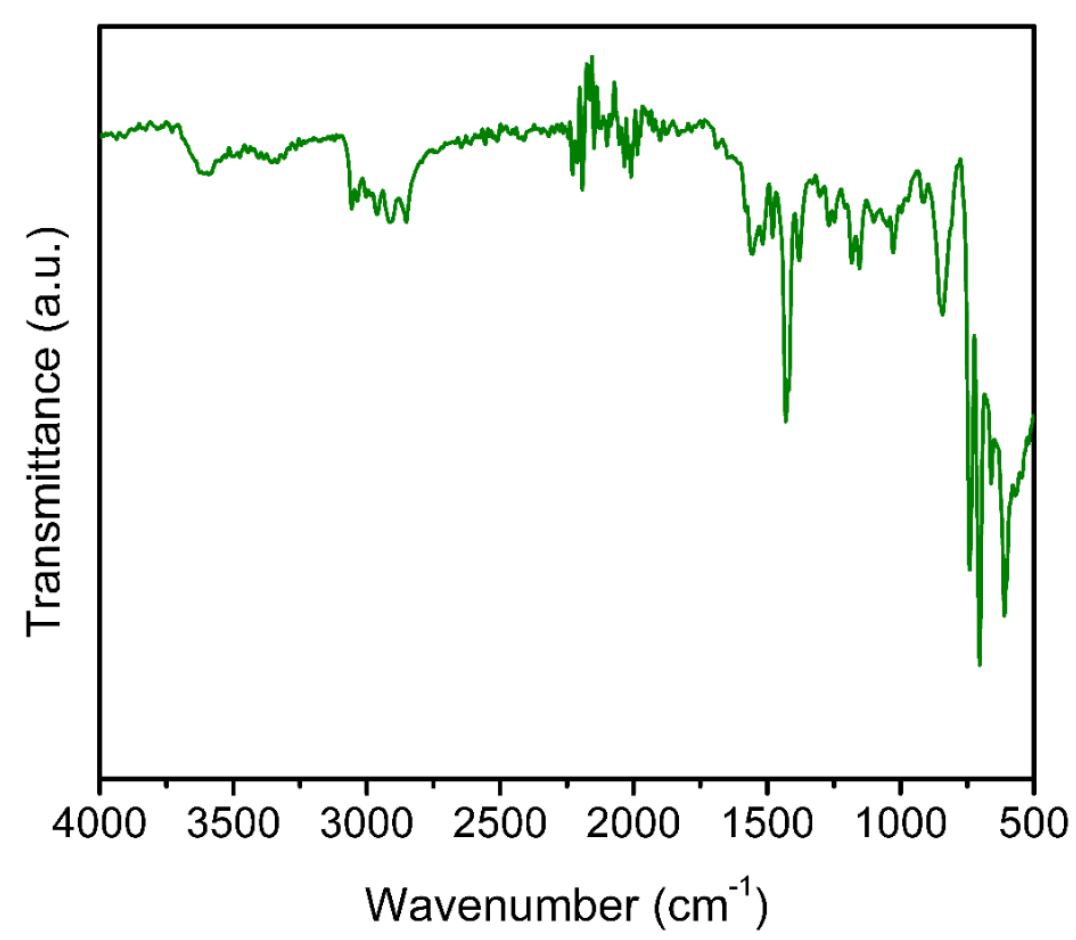

Figure S28. IR spectrum of 3-Dy. 


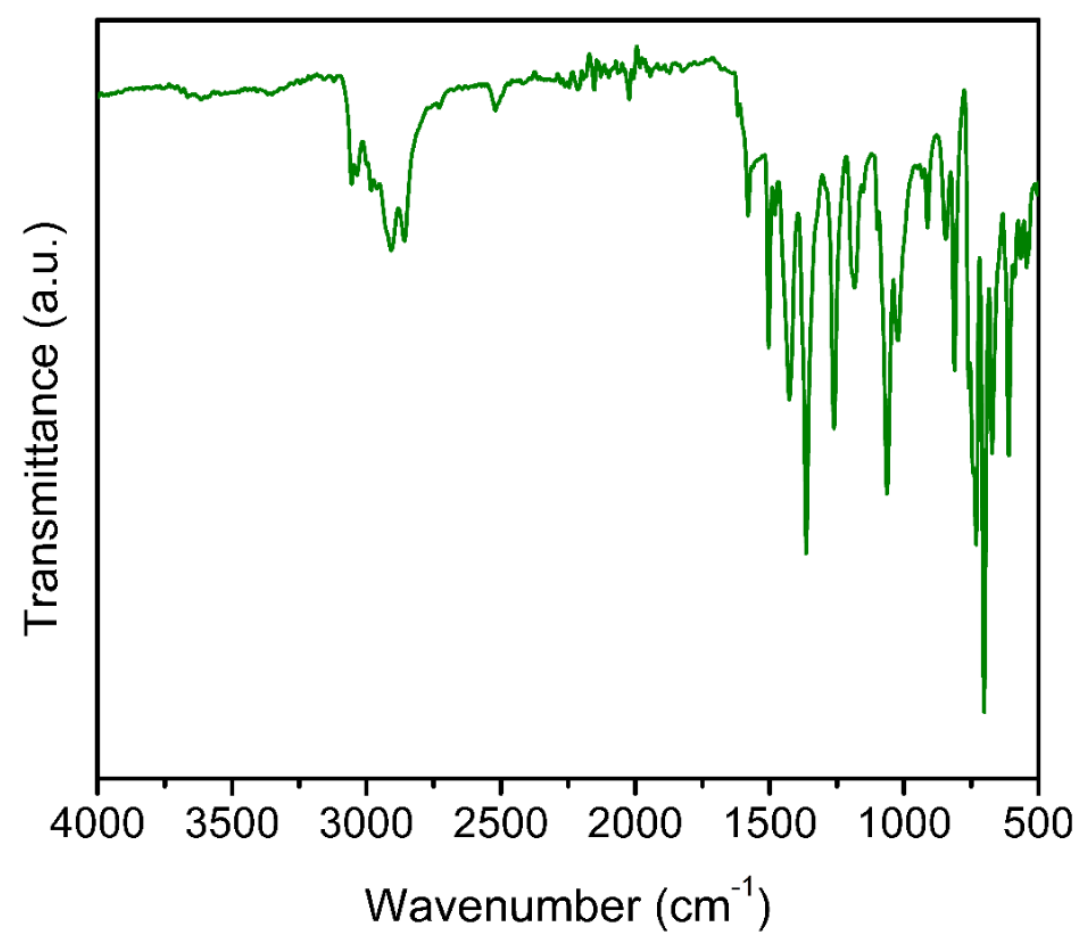

Figure S29. IR spectrum of 4-Gd.

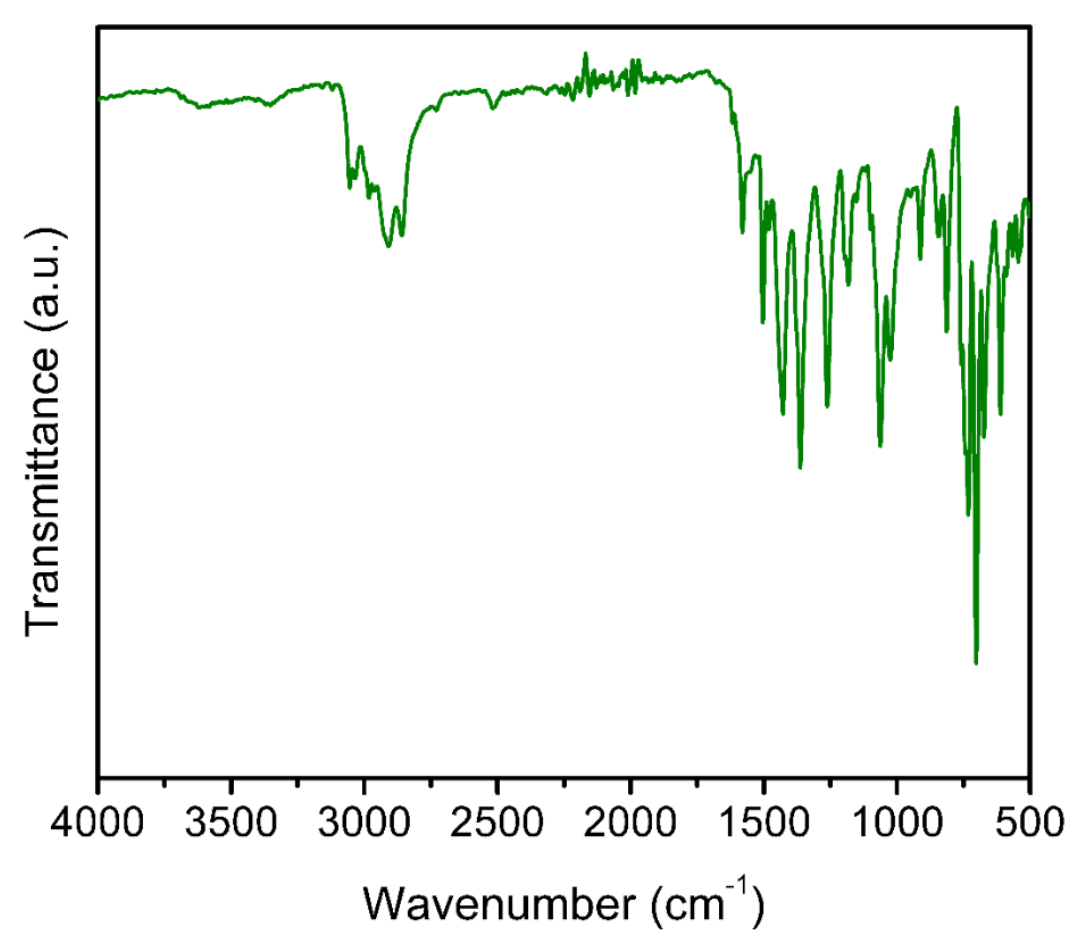

Figure S30. IR spectrum of 4-Dy. 


\section{Section S4. X-ray crystallography data refinement details $\left[\left(\mathrm{DyCp}_{2}\right)_{2}\left(\mu-5,5^{\prime}-\left(\mathrm{NMe}_{2}\right)_{2}\right.\right.$ bpym $\left.)\right]\left(\mathrm{BPh}_{4}\right)(1-\mathrm{Dy})$}

The compound crystallized in $P \overline{1}$ with two half-molecules and a $\mathrm{BPh}_{4}^{-}$counteranion in the asymmetric unit. Each Dy ion bears two Cp* rings, and one ring on each metal center is disordered over two positions. The disordered cyclopentadienyl rings were constrained to the geometrically calculated positions of ideal pentagons (AFIX 56). Anisotropic refinement of these disordered Cp* rings was stabilized by application of enhanced rigid bond restraints (RIGU). Two toluene molecules co-crystallized in the structure and each was disordered over two positions. The first toluene molecule was disordered over a crystallographic inversion center, and anisotropic displacement parameters for all seven $\mathrm{C}$ atoms were restrained to be the same (EADP). The second toluene molecule was disordered over a general position and was modeled with two positions. The phenyl ring of the disordered toluene was constrained to the ideal geometrically calculated position (AFIX 66). Anisotropic refinement of the first disordered component was achieved by using similar anisotropic displacement parameters (SIMU), while those of the second disordered component were restrained with EADP. All $\mathrm{C}\left(\mathrm{sp}^{2}\right)-\mathrm{CH}_{3}$ distances and $\mathrm{C}-\mathrm{C}-\mathrm{CH}_{3}$ angles were restrained to be similar (SADI). Two DFB molecules were also found in the structure, one of which was disordered over two positions. The phenyl ring of the disordered DFB was constrained to the ideal geometrically calculated position (AFIX 66). Anisotropic refinement of this DFB molecule was restrained by EADP. All C-F distances and $\mathrm{C}-\mathrm{C}-\mathrm{F}$ angles were restrained using SADI.

\section{$\left[\left(\mathrm{GdCp}^{*}\right)_{2}\left(\boldsymbol{\mu}-\mathbf{5 , 5} \mathbf{5}^{\prime}-(\mathrm{OEt})_{2} \mathrm{bpym}\right)\right]\left(\mathrm{BPh}_{4}\right)(\mathbf{2}-\mathrm{Gd})$}

The compound crystallized in $P 2_{1} / c$ with the whole molecule in the asymmetric unit, together with one and a half molecules of toluene. One toluene molecule was found to be disordered over a crystallographic inversion center. Refinement of this toluene molecule was stabilized by constraining the phenyl ring to be at the geometrically calculated positions (AFIX 66), and anisotropic refinement was stabilized by application of enhanced rigid bond restraints (RIGU).

\section{$\left[\left(\text { DyCp }^{*}\right)_{2}(\mu-5,5 \text { '-(OEt) })_{2}\right.$ bpym $\left.)\right]\left(\mathrm{BPh}_{4}\right)(2-\mathrm{Dy})$}

The compound crystallized in $P \overline{1}$ with the whole molecule in the asymmetric unit. One $\mathrm{Cp}^{*}$ ring showed disorder, and it was modeled over two positions. This disordered $\mathrm{Cp} *$ ring was constrained to assume the ideal geometric position using the AFIX 56 command. One phenyl ring of the $\mathrm{BPh}_{4}^{-}$ counteranion was found to be disordered over two positions, and the second component was constrained to the ideal geometric position (AFIX 66). Anisotropic refinement of these disordered components were stabilized by the application of enhanced rigid bond restraints (RIGU). In addition, one of the ethoxy groups appeared to be disordered, as suggested by unusually large thermal ellipsoids. Modeling position disorder of this group led to an unreasonable structure, and thus the disorder was not modeled. Instead, an enhanced rigid bond restraint was placed on this ethoxy group to stabilize anisotropic refinement. One molecule of toluene was found in the structure, and there exists a solvent-accessible void with a cloud of electron density. This space was located over a crystallographic inversion center, and it was modeled as a toluene molecule disordered over two positions. This disordered toluene molecule was constrained to the ideal geometric position using AFIX 66 commands, and EADP was employed during the anisotropic refinement. 


\section{$\left[\left(\mathrm{GdCp}^{*}\right)_{2}\left(\mu-5,5^{\prime}-\mathrm{Me}_{2}\right.\right.$ bpym $\left.)\right]\left(\mathrm{BPh}_{4}\right)(3-\mathrm{Gd})$}

The compound crystallized in $P 2{ }_{1} / c$ with the whole molecule in the asymmetric unit. Three of the four $\mathrm{Cp}^{*}$ rings were found to be disordered, and these rings were modeled over two positions. Anisotropic refinement of the disordered $\mathrm{Cp}^{*}$ rings was stabilized by application of enhanced rigid bond restraints (RIGU). Three THF molecules were found in the structure, and two molecules require enhanced rigid bond restraints in order to stabilize the anisotropic refinement.

\section{$\left[\left(\mathrm{DyCp}_{2}\right)_{2}\left(\mu-5,5^{\prime}-\mathrm{Me}_{2} \mathrm{bpym}\right)\right]\left(\mathrm{BPh}_{4}\right)(3-\mathrm{Dy})$}

The crystal was found to be a non-merohedral twin, and the structure was refined against both twinned domains. The compound crystallized in $P \overline{1}$ with two half-molecules in the asymmetric unit and one $\mathrm{BPh}_{4}^{-}$counteranion. Three of the four $\mathrm{Cp}^{*}$ rings were found to be disordered, and these rings were modeled over two positions. Anisotropic refinement of the disordered $\mathrm{Cp}^{*}$ rings was stabilized by application of enhanced rigid bond restraints (RIGU). Anisotropic refinement of one disordered $\mathrm{Cp}^{*}$ ring ( $\mathrm{C} 40$ to $\mathrm{C} 47$ and $\mathrm{C} 40 \mathrm{~A}$ to $\mathrm{C} 47 \mathrm{~A}$ ) cannot be stabilized by the enhanced rigid bond restraint, and thus EADP was employed on four $\mathrm{C}$ atoms (C40, C41, C45, C46, and their second disordered components). A toluene molecule was found in the asymmetric unit.

\section{$\left[\left(\mathrm{GdCp}_{2}^{*}\right)_{2}\left(\boldsymbol{\mu}-5,5^{\prime}-\mathrm{F}_{2} \mathrm{bpym}\right)\right]\left(\mathrm{BPh}_{4}\right)(4-\mathrm{Gd})$}

The compound crystallized in $P \overline{1}$ with two independent half-molecules in the asymmetric unit. Three of the four $\mathrm{Cp}^{*}$ rings were found to be disordered over two positions. Anisotropic refinement of the disordered $\mathrm{Cp}^{*}$ rings was stabilized through application of enhanced rigid bond restraints (RIGU), while constraining the sum of the occupancy to unity. One disordered $\mathrm{Cp}^{*}$ ring (C18B$\mathrm{C} 22 \mathrm{~B}$, and its disordered counterpart $\mathrm{C} 18 \mathrm{C}-\mathrm{C} 22 \mathrm{C}$ ) was constrained to the ideal planar pentagon geometry (AFIX 56) to achieve stable refinement. A 1,2-difluorobenzene solvent molecule was found to co-crystallize in the structure. It was found disordered over two positions, and anisotropic refinement was stabilized by SIMU.

\section{$\left[\left(\mathrm{DyCp}_{2}^{*}\right)_{2}\left(\boldsymbol{\mu}-5,5^{\prime}-\mathrm{F}_{2} \mathrm{bpym}\right)\right]\left(\mathrm{BPh}_{4}\right)(4-\mathrm{Dy})$}

The compound crystallized in $P \overline{1}$ with two independent half-molecules in the asymmetric unit. All four $\mathrm{Cp}^{*}$ rings were found to be disordered. Three $\mathrm{Cp}^{*}$ rings were disordered over two positions, and the other was disordered over three positions. Refinement of the disordered $\mathrm{Cp}^{*}$ rings was stabilized by the application of enhanced rigid bond restraints (RIGU). The sum of the occupancy of each Cp* ring was constrained to be unity. A 1,2-difluorobenzene solvent molecule was found to co-crystallize in the structure. It was found disordered over two positions, and anisotropic refinement was stabilized by rigid bond restraint (SIMU).

All structures display large residual electron density (up to $5 e \cdot \AA^{-3}$ ) near the lanthanide ions. We attribute this to a Fourier ripple artefact near the heavy atoms. Errors in the absorption correction, twinning, or unresolved disorder may also result in large residual electron density, however, no sign of such problems was observed. The absorption coefficient $(\mu)$ of the crystals, determined via a semi-empirical method, ranges from 1.790 to $3.187 \mathrm{~mm}^{-1}$, and thus an absorption correction error is not significant. To investigate the effect of Fourier truncation, high-angle diffraction data were removed from the refinement (Table S1). The electron density peaks and holes near the lanthanide ions move further away from the metal centers upon removal of high-angle data for all structures. Consequently, we attribute these results to an artefact from the Fourier truncation ripple. The distance of the highest peak and deepest hole in the electron density map from the lanthanide atom roughly correspond to the resolution cutoff limit (Table S1). 
Table S1. Electron density peaks and holes $\left(e \cdot \AA^{-3}\right)$ found near the Dy or Gd atoms in $\mathbf{1}-\mathbf{4}$ as a function of the resolution of the X-ray diffraction data. The corresponding distances $(\AA)$ to the lanthanide atoms are listed in parentheses.

\begin{tabular}{|c|c|c|c|c|c|c|c|}
\hline $\begin{array}{l}\text { Resolution cutoff } \\
(\AA)\end{array}$ & 0.71 & 0.77 & 0.83 & 0.90 & 1.00 & 1.10 & 1.20 \\
\hline $\begin{array}{l}\text { 1-Dy peak } \\
\text { (distance from Dy1) }\end{array}$ & $-^{a}$ & $-^{a}$ & $\begin{array}{c}5.01 \\
(1.05)\end{array}$ & $\begin{array}{c}4.52 \\
(1.08)\end{array}$ & $\begin{array}{c}3.63 \\
(1.14)\end{array}$ & $\begin{array}{c}2.70^{b} \\
(1.23)\end{array}$ & $\begin{array}{l}1.75^{b} \\
(1.45)\end{array}$ \\
\hline $\begin{array}{l}\text { 2-Gd peak } \\
\text { (distance from Gd2) } \\
\text { 2-Gd deepest hole } \\
\text { (distance from Gd2) }\end{array}$ & $\begin{array}{l}1.41 \\
(0.85) \\
-1.50 \\
(0.60)\end{array}$ & $\begin{array}{l}1.25 \\
(0.87) \\
-1.23 \\
(0.57)\end{array}$ & $\begin{array}{c}0.91 \\
(0.92) \\
-1.05 \\
(0.63)\end{array}$ & $\begin{array}{c}0.62 \\
(1.03) \\
-0.96 \\
(0.68)\end{array}$ & $\begin{array}{l}0.36 \\
(1.18) \\
-0.72 \\
(0.73)\end{array}$ & $\begin{array}{c}0.33 \\
(1.39) \\
-0.57 \\
(0.72)\end{array}$ & $\begin{array}{c}0.26 \\
(1.46) \\
-0.46 \\
(0.68)\end{array}$ \\
\hline $\begin{array}{l}\text { 2-Dy highest peak } \\
\text { (distance from Dy2) } \\
\text { 2-Dy deepest hole } \\
\text { (distance from Dy2) }\end{array}$ & $-^{a}$ & $\begin{array}{l}4.05 \\
(0.87) \\
-2.01 \\
(0.67)\end{array}$ & $\begin{array}{c}3.19 \\
(0.91) \\
-1.77 \\
(0.73)\end{array}$ & $\begin{array}{c}2.28 \\
(0.95) \\
-1.48 \\
(0.79)\end{array}$ & $\begin{array}{l}1.81 \\
(1.05) \\
-1.23 \\
(0.85)\end{array}$ & $\begin{array}{c}1.26 \\
(1.11) \\
-0.87 \\
(0.90)\end{array}$ & $\begin{array}{l}0.85^{b} \\
(1.17) \\
-0.65^{b} \\
(1.01)\end{array}$ \\
\hline $\begin{array}{l}\text { 3-Dy highest peak } \\
\text { (distance from Dy1) } \\
\text { 3-Dy deepest hole } \\
\text { (distance from Dy2) }\end{array}$ & $\begin{array}{l}3.43 \\
(0.81) \\
-2.08 \\
(0.80)\end{array}$ & $\begin{array}{c}3.13 \\
(0.91) \\
-1.65 \\
(0.83)\end{array}$ & $\begin{array}{c}2.74 \\
(0.96) \\
-1.50 \\
(0.94)\end{array}$ & $\begin{array}{l}2.30^{b} \\
(0.99) \\
-1.20^{b} \\
(1.05)\end{array}$ & $\begin{array}{l}1.72^{b} \\
(1.08) \\
-1.00^{b} \\
(1.15)\end{array}$ & $\begin{array}{l}1.36^{b} \\
(1.20) \\
-0.79^{b} \\
(1.22)\end{array}$ & $\begin{array}{l}1.03^{b} \\
(1.28) \\
-0.65^{b} \\
(1.31)\end{array}$ \\
\hline $\begin{array}{l}\text { 3-Gd peak } \\
\text { (distance from Gd2) } \\
\text { 3-Gd deepest hole } \\
\text { (distance from Gd1) }\end{array}$ & $\begin{array}{c}4.83 \\
(0.81) \\
-2.17 \\
(0.68)\end{array}$ & $\begin{array}{l}4.08 \\
(0.86) \\
-1.85 \\
(0.69)\end{array}$ & $\begin{array}{c}3.66 \\
(0.92) \\
-1.63 \\
(0.73)\end{array}$ & $\begin{array}{c}2.91 \\
(0.97) \\
-^{c}\end{array}$ & $\begin{array}{c}2.30 \\
(1.04) \\
-^{c}\end{array}$ & $\begin{array}{c}1.71 \\
(1.12) \\
-^{c}\end{array}$ & $\begin{array}{c}1.19^{b} \\
(1.20) \\
-^{c}\end{array}$ \\
\hline $\begin{array}{l}\text { 3-Gd deepest hole } \\
\text { (distance from Gd2) }\end{array}$ & $-^{c}$ & $-^{c}$ & $-^{c}$ & $\begin{array}{l}-1.26 \\
(0.76) \\
\end{array}$ & $\begin{array}{l}-1.01 \\
(0.82) \\
\end{array}$ & $\begin{array}{l}-0.81 \\
(0.92) \\
\end{array}$ & $\begin{array}{r}-0.59^{b} \\
(1.00) \\
\end{array}$ \\
\hline $\begin{array}{l}\text { 4-Gd peak } \\
\text { (distance from Gd1) }\end{array}$ & $-^{a}$ & $-^{a}$ & $\begin{array}{c}1.30 \\
(0.99)\end{array}$ & $\begin{array}{c}1.15 \\
(1.02)\end{array}$ & $\begin{array}{c}0.89 \\
(1.03)\end{array}$ & $\begin{array}{l}0.66 b \\
(1.15)\end{array}$ & $\begin{array}{l}0.46^{b} \\
(1.23)\end{array}$ \\
\hline $\begin{array}{l}\text { 4-Gd peak } \\
\text { (distance from Gd2) }\end{array}$ & $-^{a}$ & $-^{a}$ & $\begin{array}{l}1.78 \\
(0.70)\end{array}$ & $\begin{array}{c}1.41 \\
(0.77)\end{array}$ & $\begin{array}{c}0.84 \\
(0.83)\end{array}$ & $\begin{array}{l}0.51^{b} \\
(0.97)\end{array}$ & $\begin{array}{l}0.33^{b} \\
(1.03)\end{array}$ \\
\hline $\begin{array}{l}\text { 4-Gd deepest hole } \\
\text { (distance from Gd2) }\end{array}$ & $-^{a}$ & $-^{a}$ & $\begin{array}{l}-2.35 \\
(0.86) \\
\end{array}$ & $\begin{array}{l}-1.80 \\
(0.92) \\
\end{array}$ & $\begin{array}{l}-1.44 \\
(1.07) \\
\end{array}$ & $\begin{array}{r}-1.03^{b} \\
(1.13) \\
\end{array}$ & $\begin{array}{l}-0.64^{b} \\
(1.19) \\
\end{array}$ \\
\hline $\begin{array}{l}\text { 4-Dy peak } \\
\text { (distance from Dy2) }\end{array}$ & $-^{a}$ & $\begin{array}{c}2.92 \\
(0.65)\end{array}$ & $\begin{array}{c}2.26 \\
(0.67)\end{array}$ & $\begin{array}{l}1.52^{b} \\
(0.69)\end{array}$ & $\begin{array}{c}0.86^{b} \\
(0.80)\end{array}$ & $\begin{array}{l}0.69^{b} \\
(0.97)\end{array}$ & $\begin{array}{r}0.44^{b} \\
(1.05)\end{array}$ \\
\hline $\begin{array}{l}\text { 4-Dy peak } \\
\text { (distance from Dy1) }\end{array}$ & $-^{a}$ & $\begin{array}{c}2.44 \\
(0.93)\end{array}$ & $\begin{array}{c}2.34 \\
(0.95)\end{array}$ & $\begin{array}{l}2.16^{b} \\
(0.98)\end{array}$ & $\begin{array}{l}1.65^{b} \\
(1.03)\end{array}$ & $\begin{array}{r}1.08^{b} \\
(1.14)\end{array}$ & $\begin{array}{r}0.73^{b} \\
(1.23)\end{array}$ \\
\hline $\begin{array}{l}\text { 4-Dy deepest hole } \\
\text { (distance from Dy2) }\end{array}$ & $-^{a}$ & $\begin{array}{l}-3.36 \\
(0.84)\end{array}$ & $\begin{array}{l}-2.5 \\
(0.88)\end{array}$ & $\begin{array}{l}-1.63^{b} \\
(0.98)\end{array}$ & $\begin{array}{r}-1.36^{b} \\
(1.09)\end{array}$ & $-^{c}$ & $-^{c}$ \\
\hline
\end{tabular}

${ }^{a}$ Refinement did not converge due to poor resolution. ${ }^{b}$ Data are not included due to poor signal-tonoise ratio. ${ }^{c}$ Only the deepest holes are extracted from the refinement. Some data are missing because different holes emerge. For 1-Dy, the deepest hole is located near a disordered 1,2difluorobenzene molecule, presumably due to partial occupation of the solvent molecule which is modeled assuming full occupancy. 
Table S2. Crystal data and structure refinement for $\left[\left(\mathrm{DyCp}_{2}\right)_{2}\left(\mu-5,5^{\prime}-\left(\mathrm{NMe}_{2}\right)_{2} \mathrm{bpym}\right)\right]\left(\mathrm{BPh}_{4}\right)(\mathbf{1 -}$ Dy).

\begin{tabular}{ll}
\hline CCDC number & 2035540 \\
Empirical formula & $\mathrm{Dy}_{2} \mathrm{~F}_{4} \mathrm{~N}_{6} \mathrm{C}_{98.5} \mathrm{BH}_{116}$ \\
Formula weight $(\mathrm{g} / \mathrm{mol})$ & 1795.78 \\
Temperature $(\mathrm{K})$ & $100(2)$ \\
Wavelength $(\AA)$ & 0.71073 \\
Crystal system & Triclinic \\
Space group & $P \overline{1}$ \\
$a(\AA), \alpha\left(^{\circ}\right)$ & $11.4042(4), 69.062(3)$ \\
$b(\AA), \beta\left(^{\circ}\right)$ & $20.0634(7), 75.923(3)$ \\
$c(\AA), \gamma\left({ }^{\circ}\right)$ & $21.0569(7), 74.924(3)$ \\
Volume $\left(\AA^{3}\right)$ & $4284.2(3)$ \\
$Z$ & 2 \\
Density (calculated, $\left.\mathrm{g} / \mathrm{cm}^{3}\right)$ & 1.392 \\
Absorption coefficient $\left(\mathrm{mm}^{-1}\right)$ & 1.790 \\
$F(000)$ & 1844 \\
Crystal size (mm $\left.{ }^{3}\right)$ & $0.25 \times 0.084 \times 0.046$ \\
$\theta$ range for data collection $\left({ }^{\circ}\right)$ & 2.994 to 25.350 \\
Index ranges & $-13 \leq h \leq 13$ \\
& $-24 \leq k \leq 24$ \\
Reflections collected & $-25 \leq l \leq 25$ \\
Independent reflections, $R_{\text {int }}$ & 77485 \\
Completeness to $\theta=25.242^{\circ}$ & $15675,0.0923$ \\
Absorption correction & $99.8 \%$ \\
Max. and min. transmission & Semi-empirical \\
Refinement method & 1.000 and 0.61089 \\
Data / restraints / parameters & Full-matrix least-squares on $F^{2}$ \\
Goodness-of-fit on $F^{2}$ & $15675 / 564 / 1140$ \\
$R$ indices (all data) & 1.143 \\
Extinction coefficient & $R_{1}=0.0830, w R_{2}=0.2047$ \\
Largest diff. peak and hole $\left(e \cdot \AA^{-3}\right)$ & $R_{1}=0.1029, w R_{2}=0.2146$ \\
\hline
\end{tabular}


Table S3. Crystal data and structure refinement for $\left[\left(\mathrm{GdCp}_{2}\right)_{2}\left(\mu-5,5^{\prime}-(\mathrm{OEt})_{2} \mathrm{bpym}\right)\right]\left(\mathrm{BPh}_{4}\right)$ (2Gd) and $\left[\left(\mathrm{DyCp}^{*}{ }_{2}\right)_{2}\left(\mu-5,5^{\prime}-(\mathrm{OEt})_{2} \mathrm{bpym}\right)\right]\left(\mathrm{BPh}_{4}\right)(\mathbf{2}-\mathrm{Dy})$.

\begin{tabular}{|c|c|c|}
\hline & 2-Gd & 2-Dy \\
\hline CCDC number & 2035544 & 2035539 \\
\hline Empirical formula & $\mathrm{Gd}_{2} \mathrm{O}_{2} \mathrm{~N}_{4} \mathrm{C}_{86.5} \mathrm{BH}_{106}$ & $\mathrm{Dy}_{2} \mathrm{O}_{2} \mathrm{~N}_{4} \mathrm{C}_{86.5} \mathrm{BH}_{106}$ \\
\hline Formula weight (g/mol) & 1559.06 & 1569.56 \\
\hline Temperature (K) & $100(2)$ & $100(2)$ \\
\hline Wavelength $(\AA)$ & 0.8856 & 0.7288 \\
\hline Crystal system & Monoclinic & Triclinic \\
\hline Space group & $P 2_{1} / c$ & $P \overline{1}$ \\
\hline$a(\AA), \alpha\left(^{\circ}\right)$ & $12.192(2), 90$ & $10.9649(17), 89.336(2)$ \\
\hline$b(\AA), \beta\left(^{\circ}\right)$ & $24.487(5), 101.198(4)$ & $16.592(3), 85.526(2)$ \\
\hline$c(\AA), \gamma\left({ }^{\circ}\right)$ & $25.717(5), 90$ & $21.571(4), 85.424(3)$ \\
\hline Volume $\left(\AA^{3}\right)$ & $7531(2)$ & $3900.0(11)$ \\
\hline$Z$ & 4 & 2 \\
\hline Density (calculated, $\mathrm{g} / \mathrm{cm}^{3}$ ) & 1.375 & 1.337 \\
\hline Absorption coefficient $\left(\mathrm{mm}^{-1}\right)$ & 3.187 & 2.072 \\
\hline$F(000)$ & 3208 & 1612 \\
\hline Crystal size $\left(\mathrm{mm}^{3}\right)$ & $0.071 \times 0.036 \times 0.028$ & $0.11 \times 0.086 \times 0.050$ \\
\hline$\theta$ range for data collection $\left(^{\circ}\right)$ & 2.122 to 38.566 & 2.080 to 28.245 \\
\hline \multirow[t]{3}{*}{ Index ranges } & $-16 \leq h \leq 17$ & $-14 \leq h \leq 14$ \\
\hline & $-34 \leq k \leq 34$ & $-21 \leq k \leq 21$ \\
\hline & $-36 \leq l \leq 35$ & $-28 \leq l \leq 28$ \\
\hline Reflections collected & 107187 & 70841 \\
\hline Independent reflections, $R_{\text {int }}$ & $21908,0.0502$ & $17878,0.0608$ \\
\hline Completeness to $\theta$ & $100.0 \%\left(\theta=32.097^{\circ}\right)$ & $100.0 \%\left(\theta=25.930^{\circ}\right)$ \\
\hline Absorption correction & Semi-empirical & Semi-empirical \\
\hline Max. and min. transmission & 0.916 and 0.757 & 0.903 and 0.742 \\
\hline Refinement method & Full-matrix least-squares on $F^{2}$ & Full-matrix least-squares on $F^{2}$ \\
\hline Data / restraints / parameters & 21908 / 45 / 902 & $17878 / 247 / 983$ \\
\hline Goodness-of-fit on $F^{2}$ & 1.083 & 1.051 \\
\hline Final $R$ indices $[I>2 \sigma(I)]$ & $R_{1}=0.0398, w R_{2}=0.0911$ & $R_{1}=0.0490, w R_{2}=0.1240$ \\
\hline$R$ indices (all data) & $R_{1}=0.0648, w R_{2}=0.0997$ & $R_{1}=0.0686, w R_{2}=0.1366$ \\
\hline Extinction coefficient & $\mathrm{n} / \mathrm{a}$ & $\mathrm{n} / \mathrm{a}$ \\
\hline Largest diff. peak and hole $\left(e \cdot \AA^{-3}\right)$ & 1.702 and -1.496 & 4.067 and -2.005 \\
\hline
\end{tabular}


Table S4. Crystal data and structure refinement for $\left[\left(\mathrm{GdCp}_{2}\right)_{2}\left(\mu-5,5^{\prime}-\mathrm{Me}_{2} \mathrm{bpym}\right)\right]\left(\mathrm{BPh}_{4}\right)(\mathbf{3 - G d})$ and $\left[\left(\mathrm{DyCp}^{*}\right)_{2}\left(\mu-5,5^{\prime}-\mathrm{Me}_{2}\right.\right.$ bpym $\left.)\right]\left(\mathrm{BPh}_{4}\right)(3-\mathrm{Dy})$.

\begin{tabular}{|c|c|c|}
\hline & 3-Gd & 3-Dy \\
\hline CCDC number & 2035541 & 2035538 \\
\hline Empirical formula & $\mathrm{Gd}_{2} \mathrm{O}_{3} \mathrm{~N}_{4} \mathrm{C}_{86} \mathrm{BH}_{114}$ & $\mathrm{Dy}_{2} \mathrm{~N}_{4} \mathrm{C}_{81} \mathrm{BH}_{98}$ \\
\hline Formula weight $(\mathrm{g} / \mathrm{mol})$ & 1577.12 & 1463.44 \\
\hline Temperature $(\mathrm{K})$ & $100(2)$ & $100(2)$ \\
\hline Wavelength $(\AA)$ & 0.7749 & 0.7749 \\
\hline Crystal system & Monoclinic & Triclinic \\
\hline Space group & $P 2_{1} / c$ & $P \overline{1}$ \\
\hline$a(\AA), \alpha\left(^{\circ}\right)$ & $12.549(3), 90$ & $12.2665(7), 84.427(3)$ \\
\hline$b(\AA), \beta\left(^{\circ}\right)$ & $27.027(6), 91.819(5)$ & $12.8149(8), 83.779(4)$ \\
\hline$c(\AA), \gamma\left({ }^{\circ}\right)$ & $22.460(6), 90$ & $22.0900(13), 85.473(4)$ \\
\hline Volume $\left(\AA^{3}\right)$ & $7614(3)$ & $3427.4(4)$ \\
\hline$Z$ & 4 & 2 \\
\hline Density (calculated, $\mathrm{g} / \mathrm{cm}^{3}$ ) & 1.376 & 1.418 \\
\hline Absorption coefficient $\left(\mathrm{mm}^{-1}\right)$ & 2.210 & 2.748 \\
\hline$F(000)$ & 3260 & 1498 \\
\hline Crystal size $\left(\mathrm{mm}^{3}\right)$ & & $0.071 \times 0.054 \times 0.036$ \\
\hline$\theta$ range for data collection $\left(^{\circ}\right)$ & 2.142 to 33.073 & 2.096 to 27.827 \\
\hline \multirow[t]{3}{*}{ Index ranges } & $-17 \leq h \leq 17$ & $-14 \leq h \leq 14$ \\
\hline & $-38 \leq k \leq 38$ & $-15 \leq k \leq 15$ \\
\hline & $-31 \leq l \leq 31$ & $0 \leq l \leq 26$ \\
\hline Reflections collected & 128659 & 14858 \\
\hline Independent reflections, $R_{\text {int }}$ & $22278,0.0564$ & $12511,0.1733$ \\
\hline Completeness to $\theta=27.706^{\circ}$ & $99.9 \%$ & $99.6 \%$ \\
\hline Absorption correction & Semi-empirical & Semi-empirical \\
\hline Max. and min. transmission & 0.9705 and 0.8958 & 0.908 and 0.783 \\
\hline Refinement method & Full-matrix least-squares on $F^{2}$ & Full-matrix least-squares on $F^{2}$ \\
\hline Data / restraints / parameters & $22278 / 522 / 1153$ & $12511 / 450 / 1032$ \\
\hline Goodness-of-fit on $F^{2}$ & 1.034 & 1.099 \\
\hline Final $R$ indices $[I>2 \sigma(I)]$ & $R_{1}=0.0536, w R_{2}=0.1340$ & $R_{1}=0.0621, w R_{2}=0.1368$ \\
\hline$R$ indices (all data) & $R_{1}=0.0801, w R_{2}=0.1466$ & $R_{1}=0.0978, w R_{2}=0.1497$ \\
\hline Extinction coefficient & $\mathrm{n} / \mathrm{a}$ & $\mathrm{n} / \mathrm{a}$ \\
\hline Largest diff. peak and hole $\left(e \cdot \AA^{-3}\right)$ & 4.961 and -2.170 & 2.762 and -1.500 \\
\hline
\end{tabular}


Table S5. Crystal data and structure refinement for $\left[\left(\mathrm{GdCp}_{2}\right)_{2}\left(\mu-5,5^{\prime}-\mathrm{F}_{2} \mathrm{bpym}\right)\right]\left(\mathrm{BPh}_{4}\right)(4-\mathrm{Gd})$ and $\left[\left(\mathrm{DyCp}^{*}\right)_{2}\left(\mu-5,5^{\prime}-\mathrm{F}_{2} \mathrm{bpym}\right)\right]\left(\mathrm{BPh}_{4}\right)(4-\mathrm{Dy})$.

\begin{tabular}{|c|c|c|}
\hline & 4-Gd & 4-Dy \\
\hline CCDC number & 2035542 & 2035543 \\
\hline Empirical formula & $\mathrm{Gd}_{2} \mathrm{~F}_{4} \mathrm{~N}_{4} \mathrm{C}_{78} \mathrm{BH}_{88}$ & $\mathrm{Dy}_{2} \mathrm{~F}_{4} \mathrm{~N}_{4} \mathrm{C}_{78} \mathrm{BH}_{88}$ \\
\hline Formula weight (g/mol) & 1482.83 & 1493.33 \\
\hline Temperature (K) & $100(2)$ & $100(2)$ \\
\hline Wavelength $(\AA)$ & 0.7288 & 0.7288 \\
\hline Crystal system & Triclinic & Triclinic \\
\hline Space group & $P \overline{1}$ & $P \overline{1}$ \\
\hline$a(\AA), \alpha\left(^{\circ}\right)$ & $11.604(4), 80.776(5)$ & $11.615(2), 80.648(2)$ \\
\hline$b(\AA), \beta\left(^{\circ}\right)$ & $13.026(4), 85.537(5)$ & 12.991(2), 85.621(2) \\
\hline$c(\AA), \gamma\left({ }^{\circ}\right)$ & $22.654(8), 87.739(6)$ & $22.607(4), 87.720(3)$ \\
\hline Volume $\left(\AA^{3}\right)$ & $3368.3(19)$ & $3354.7(10)$ \\
\hline$Z$ & 2 & 2 \\
\hline Density (calculated, $\mathrm{g} / \mathrm{cm}^{3}$ ) & 1.462 & 1.478 \\
\hline Absorption coefficient $\left(\mathrm{mm}^{-1}\right)$ & 2.138 & 2.411 \\
\hline$F(000)$ & 1506 & 1514 \\
\hline Crystal size $\left(\mathrm{mm}^{3}\right)$ & $0.13 \times 0.07 \times 0.064$ & $0.10 \times 0.086 \times 0.020$ \\
\hline$\theta$ range for data collection $\left({ }^{\circ}\right)$ & 2.094 to 25.708 & 1.630 to 28.244 \\
\hline \multirow[t]{3}{*}{ Index ranges } & $-13 \leq h \leq 13$ & $-15 \leq h \leq 15$ \\
\hline & $-15 \leq k \leq 15$ & $-16 \leq k \leq 16$ \\
\hline & $-26 \leq l \leq 26$ & $-29 \leq l \leq 29$ \\
\hline Reflections collected & 50086 & 59794 \\
\hline Independent reflections, $R_{\text {int }}$ & $11889,0.0718$ & $15374,0.0581$ \\
\hline Completeness to $\theta$ & $99.9 \%\left(\theta=25.708^{\circ}\right)$ & $100.0 \%\left(\theta=25.930^{\circ}\right)$ \\
\hline Absorption correction & Semi-empirical & Semi-empirical \\
\hline Max. and min. transmission & 0.875 and 0.757 & 0.953 and 0.837 \\
\hline Refinement method & Full-matrix least-squares on $F^{2}$ & Full-matrix least-squares on $F^{2}$ \\
\hline Data / restraints / parameters & $11889 / 702 / 1106$ & 15374 / $997 / 1376$ \\
\hline Goodness-of-fit on $F^{2}$ & 1.076 & 1.188 \\
\hline Final $R$ indices $[I>2 \sigma(I)]$ & $R_{1}=0.0545, w R_{2}=0.1042$ & $R_{1}=0.0657, w R_{2}=0.1389$ \\
\hline$R$ indices (all data) & $R_{1}=0.0751, w R_{2}=0.1151$ & $R_{1}=0.0817, w R_{2}=0.1456$ \\
\hline Extinction coefficient & $\mathrm{n} / \mathrm{a}$ & $\mathrm{n} / \mathrm{a}$ \\
\hline Largest diff. peak and hole $\left(e \cdot \AA^{-3}\right)$ & 1.789 and -2.336 & 2.922 and -3.356 \\
\hline
\end{tabular}




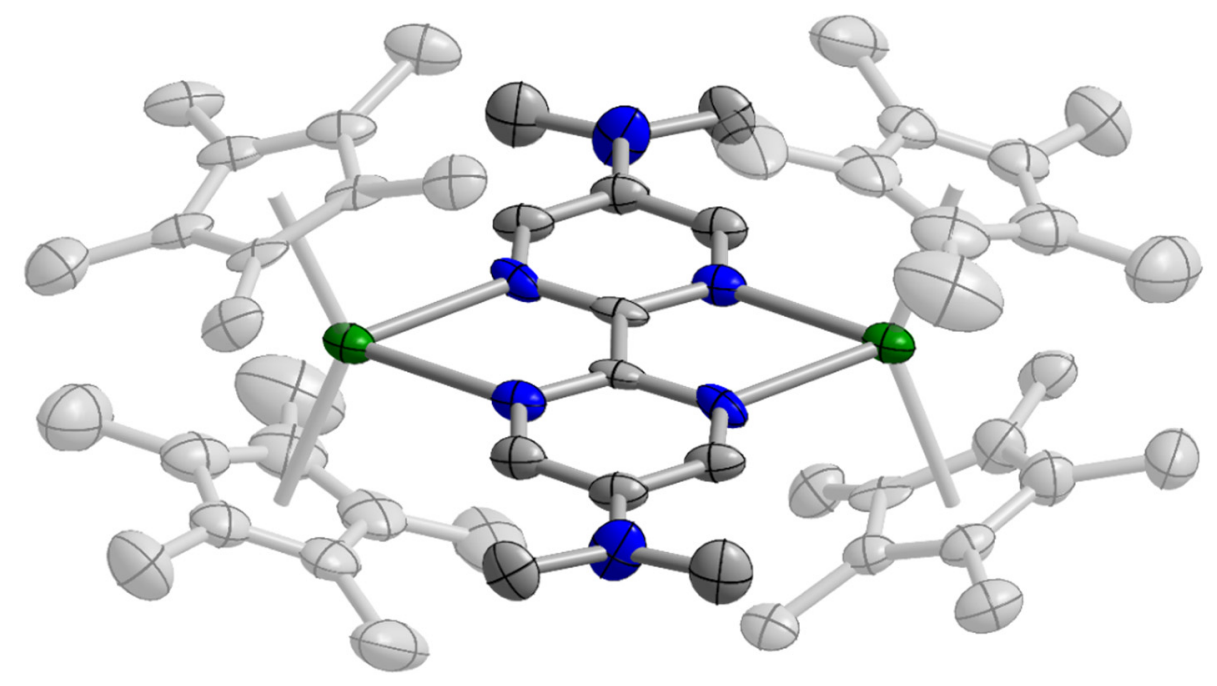

Figure S31. Solid-state structure of one of the $\left[\left(\mathrm{DyCp}^{*}{ }_{2}\right)_{2}\left(\mu-5,5^{\prime}-\left(\mathrm{NMe}_{2}\right)_{2} \text { bpym }\right)\right]^{+}$complex cations in 1-Dy with thermal ellipsoids at the $50 \%$ probability level. Hydrogen atoms, the $\mathrm{BPh}_{4}{ }^{-}$ counteranion, solvent molecules, and the second $\left[\left(\mathrm{DyCp}^{*}{ }_{2}\right)_{2}\left(\mu-5,5^{\prime}-\left(\mathrm{NMe}_{2}\right)_{2} \text { bpym }\right)\right]^{+}$cation are omitted for clarity. Green, blue, and grey ellipsoids represent Dy, N, and C atoms, respectively.

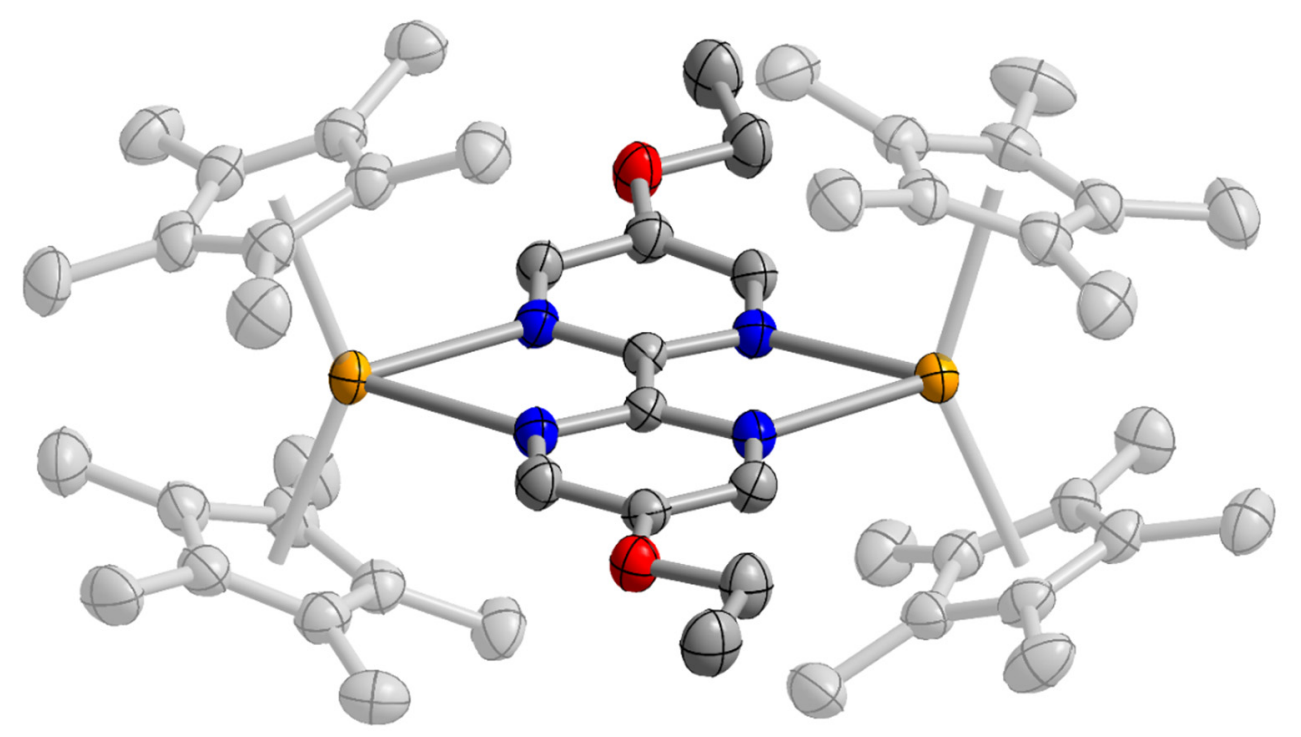

Figure S32. Solid-state structure of the $\left[\left(\mathrm{GdCp}^{*}{ }_{2}\right)_{2}\left(\mu-5,5^{\prime}-(\mathrm{OEt})_{2} \mathrm{bpym}\right)\right]^{+}$cation in 2-Gd with thermal ellipsoids at the $50 \%$ probability level. Hydrogen atoms, the $\mathrm{BPh}_{4}{ }^{-}$counteranion, and solvent molecules are omitted for clarity. Orange, blue, red, and grey ellipsoids represent $\mathrm{Gd}, \mathrm{N}$, $\mathrm{O}$, and $\mathrm{C}$ atoms, respectively. 


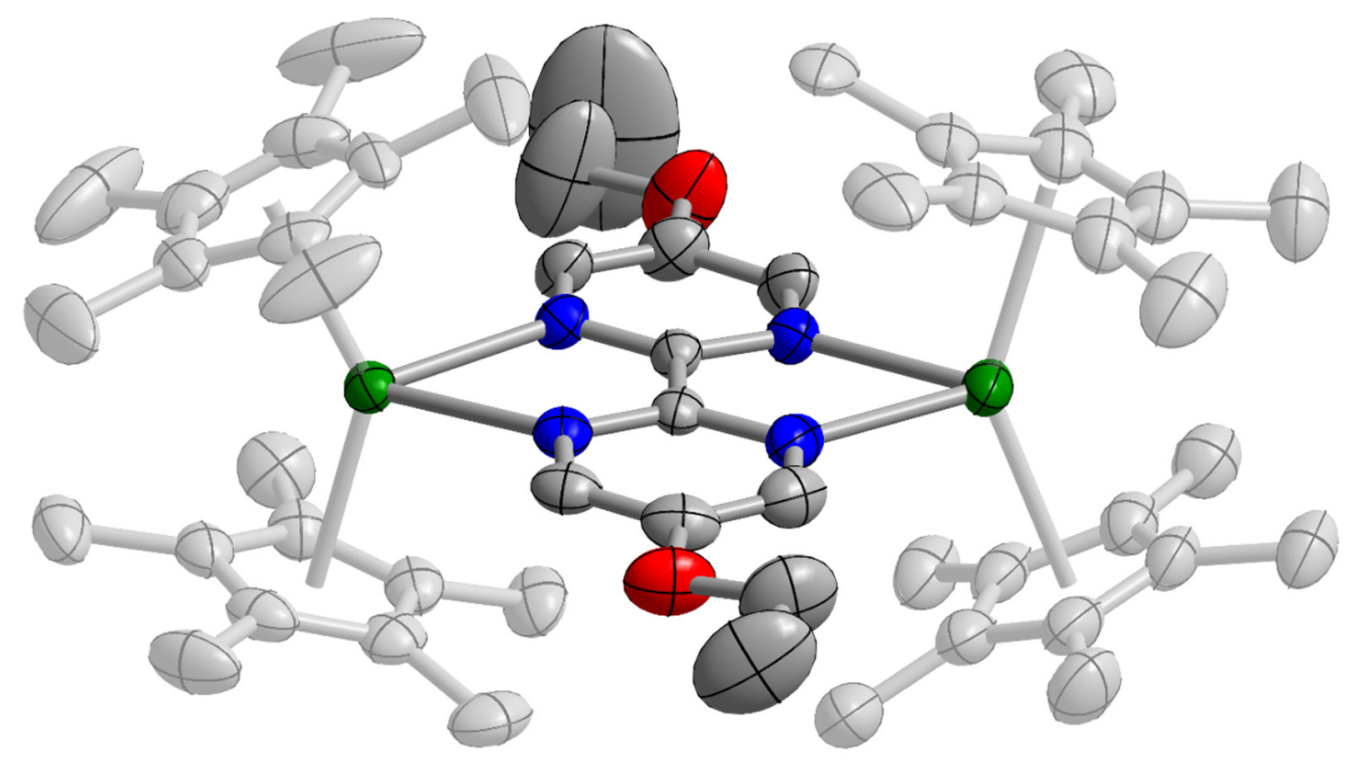

Figure S33. Solid-state structure of the $\left[\left(\mathrm{DyCp}_{2}{ }_{2}\right)_{2}\left(\mu-5,5^{\prime}-(\mathrm{OEt})_{2} \mathrm{bpym}\right)\right]^{+}$cation in 2-Dy with thermal ellipsoids at the $50 \%$ probability level. Hydrogen atoms, the $\mathrm{BPh}^{-}$counteranion, and solvent molecules are omitted for clarity. The terminal carbon atom in the ethoxy group shows a large thermal ellipsoid due to rotational disorder that could not be modelled. Green, blue, red, and grey ellipsoids represent $\mathrm{Dy}, \mathrm{N}, \mathrm{O}$, and $\mathrm{C}$ atoms, respectively.

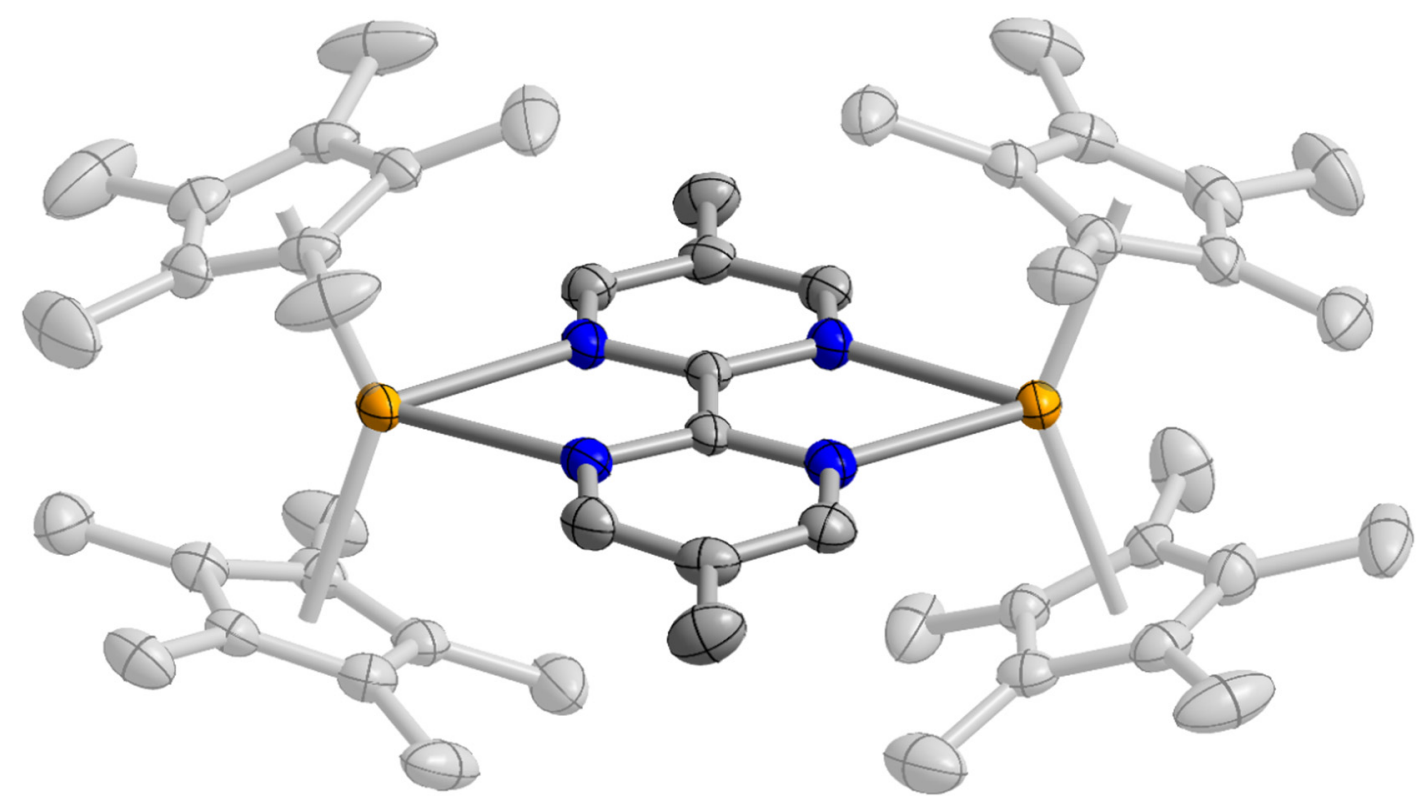

Figure S34. Solid-state structure of the $\left[\left(\mathrm{GdCp}^{*}{ }_{2}\right) 2\left(\mu-5,5^{\prime}-\mathrm{Me} 2 \mathrm{bpym}\right)\right]^{+}$cation in 3-Gd with thermal ellipsoids at the $50 \%$ probability level. Hydrogen atoms, the $\mathrm{BPh}_{4}{ }^{-}$counteranion, and solvent molecules are omitted for clarity. Orange, blue, and grey ellipsoids represent $\mathrm{Gd}, \mathrm{N}$, and $\mathrm{C}$ atoms, respectively. 


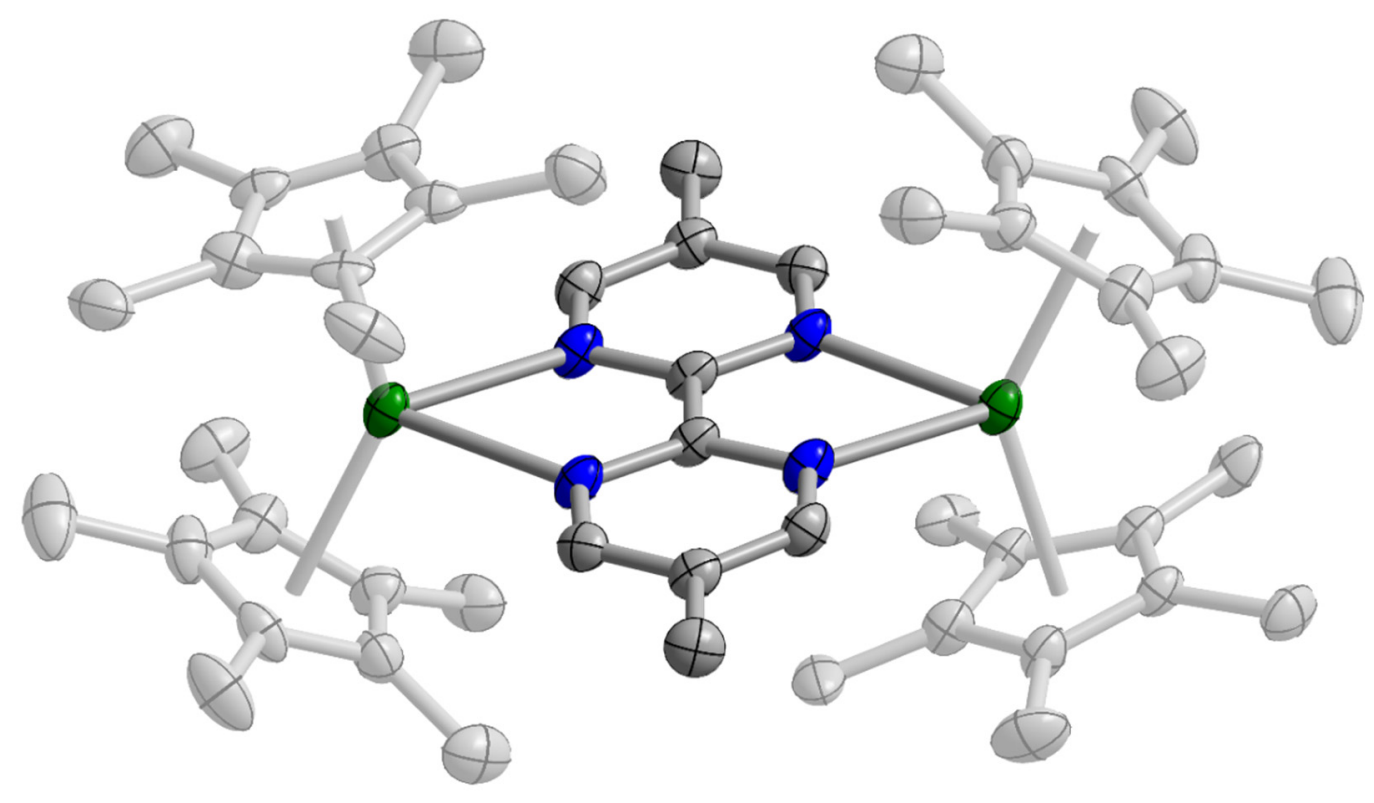

Figure S35. Solid-state structure of one of the $\left[\left(\mathrm{DyCp}^{*}{ }_{2}\right)_{2}\left(\mu-5,5^{\prime}-\mathrm{Me}{ }_{2} \text { bpym }\right)\right]^{+}$cations in 3-Dy with thermal ellipsoids at the $50 \%$ probability level. Hydrogen atoms, the $\mathrm{BPh}_{4}^{-}$counteranion, solvent molecules, and the second $\left[\left(\mathrm{DyCp}^{*}\right)_{2}\left(\mu-5,5^{\prime}-\mathrm{Me} 2 \mathrm{bpym}\right)\right]^{+}$cation are omitted for clarity. Green, blue, and grey ellipsoids represent $\mathrm{Dy}, \mathrm{N}$, and $\mathrm{C}$ atoms, respectively.

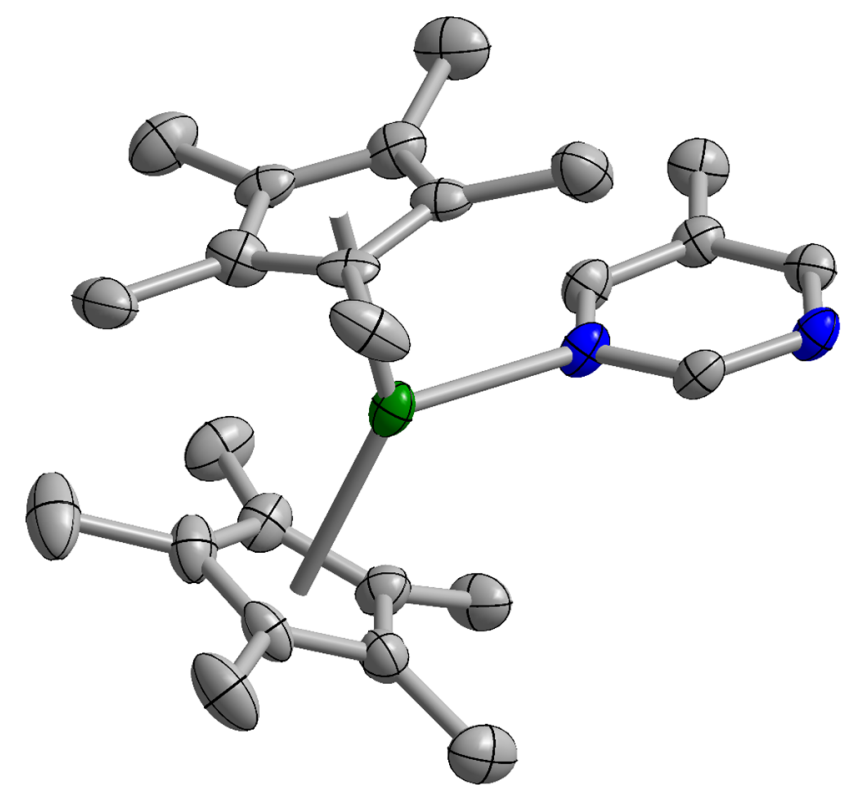

Figure S36. Asymmetric unit of the solid-state structure of one of the $\left[\left(\mathrm{DyCp}_{2}^{*}\right)_{2}\left(\mu-5,5^{\prime}-\right.\right.$ $\mathrm{Me}_{2}$ bpym) $]^{+}$cations in 3-Dy with thermal ellipsoids at the $50 \%$ probability level. Hydrogen atoms, the $\mathrm{BPh}_{4}{ }^{-}$counteranion, solvent molecules, and the second $\left[\left(\mathrm{DyCp}_{2}{ }_{2}\right)_{2}\left(\mu-5,5^{\prime}-\mathrm{Me}_{2} \text { bpym }\right)\right]^{+}$cation are omitted for clarity. Green, blue, and grey ellipsoids represent Dy, N, and C atoms, respectively. 


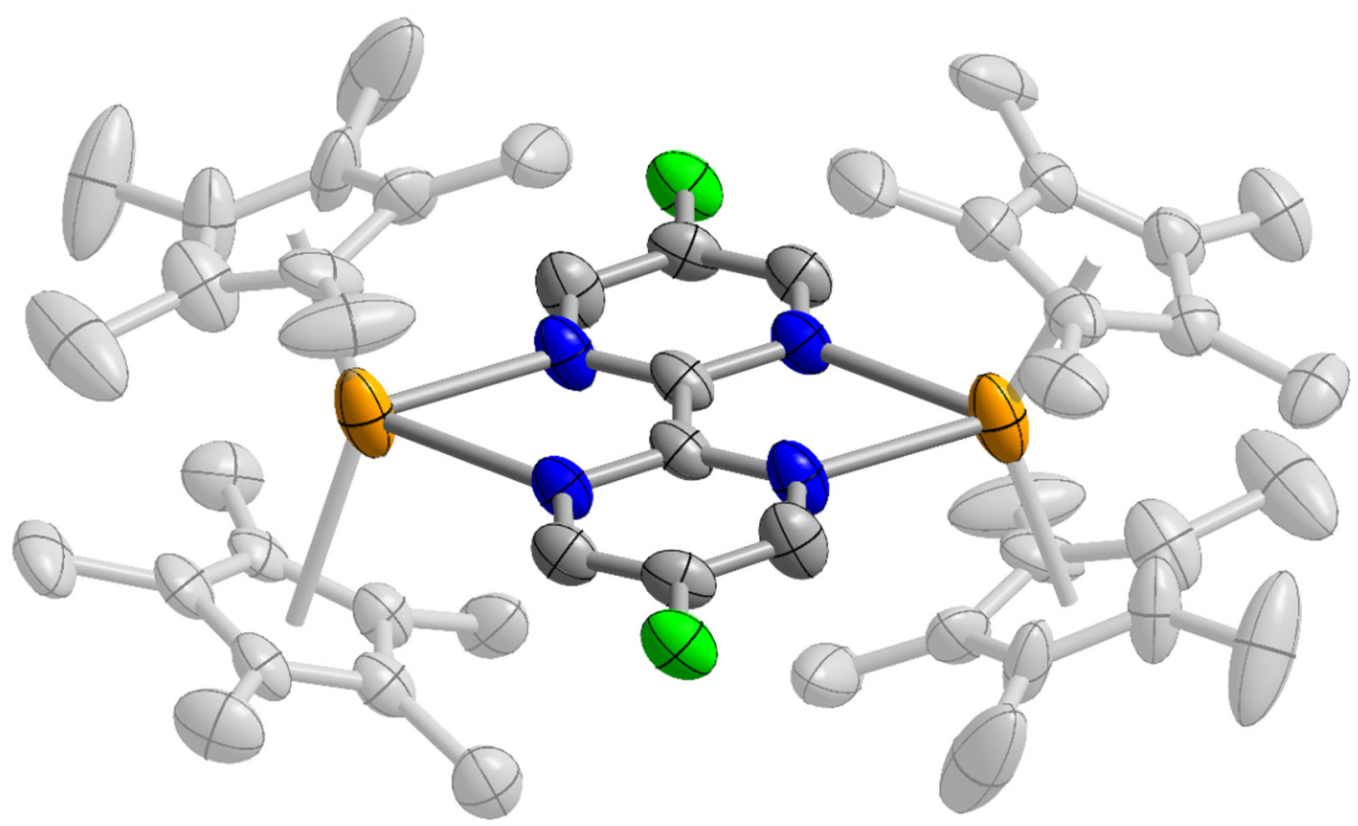

Figure S37. Solid-state structure of one of the $\left[\left(\mathrm{GdCp}_{2}{ }_{2}\right)_{2}\left(\mu-5,5^{\prime}-\mathrm{F}_{2} \text { bpym }\right)\right]^{+}$cations in 4-Gd with thermal ellipsoids at the $50 \%$ probability level. Hydrogen atoms, the $\mathrm{BPh}_{4}{ }^{-}$counteranion, solvent molecules, and the second $\left[\left(\mathrm{GdCp}_{2}{ }_{2}\right)_{2}\left(\mu-5,5^{\prime}-\mathrm{F}_{2} \mathrm{bpym}\right)\right]^{+}$cation are omitted for clarity. Orange, blue, lime green, and grey ellipsoids represent $\mathrm{Gd}, \mathrm{N}, \mathrm{F}$, and $\mathrm{C}$ atoms, respectively.

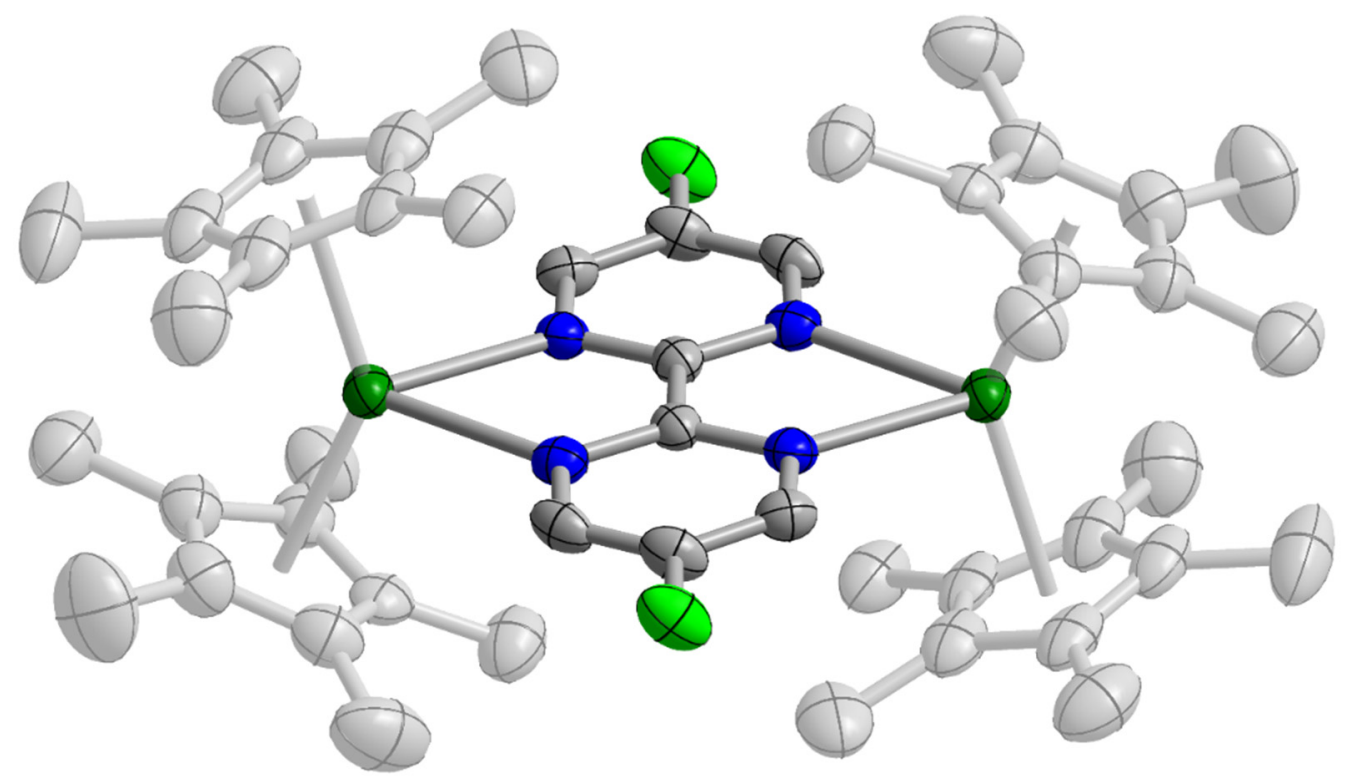

Figure S38. Solid-state structure of one of the $\left[\left(\mathrm{DyCp}^{*}\right)_{2}\left(\mu-5,5^{\prime}-\mathrm{F}_{2} \text { bpym }\right)\right]^{+}$cations in 4-Dy with thermal ellipsoids at the $50 \%$ probability level. Hydrogen atoms, the $\mathrm{BPh}_{4}{ }^{-}$counteranion, solvent molecules, and the second $\left[\left(\mathrm{DyCp}^{*}\right)_{2}\left(\mu-5,5^{\prime}-\mathrm{F}_{2} \text { bpym }\right)\right]^{+}$cation are omitted for clarity. Green, blue, lime green, and grey ellipsoids represent Dy, N, F, and C atoms, respectively. 


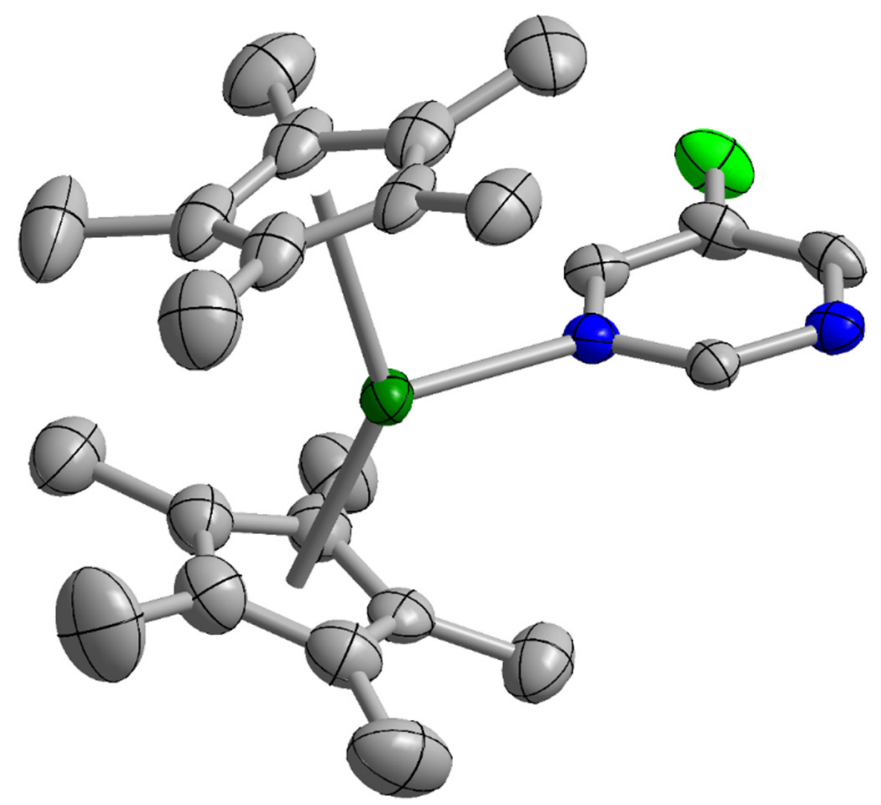

Figure S39. Asymmetric unit of the solid-state structure of one of the $\left[\left(\mathrm{DyCp}_{2}\right)_{2}\left(\mu-5,5^{\prime}-\mathrm{F}_{2} \mathrm{bpym}\right)\right]^{+}$ cations in 4-Dy with thermal ellipsoids at the $50 \%$ probability level. Hydrogen atoms, the $\mathrm{BPh}_{4}^{-}$ counteranion, solvent molecules, and the second $\left[\left(\mathrm{DyCp}_{2}{ }_{2}\right)_{2}\left(\mu-5,5^{\prime}-\mathrm{F}_{2} \text { bpym }\right)\right]^{+}$cation are omitted for clarity. Green, blue, lime green, and grey ellipsoids represent Dy, N, F, and C atoms, respectively.

Table S6. Selected bond distances $(\AA)$ and angles $\left(^{\circ}\right)$ for 2-Gd, 3-Gd, and 4-Gd.

\begin{tabular}{|c|c|c|c|}
\hline & 2-Gd & 3-Gd & 4-Gd ${ }^{a}$ \\
\hline Gd1-N1 & $2.471(2)$ & $2.462(4)$ & $2.468(5)$ \\
\hline Gd1-N1' & $2.481(2)$ & $2.476(4)$ & - \\
\hline Gd2-N3 & $2.446(2)$ & $2.450(4)$ & $2.488(5)$ \\
\hline Gd2-N3' & $2.432(2)$ & $2.457(4)$ & - \\
\hline Gd1B-N1B & - & - & $2.474(5)$ \\
\hline Gd1B-N3B & - & - & $2.446(5)$ \\
\hline $\mathrm{Gd}-\mathrm{N}($ avg $)$ & $2.458(1)^{b}$ & $2.461(2)^{b}$ & $2.469(3)$ \\
\hline $\mathrm{C} 2-\mathrm{C} 2^{\prime}$ & $1.416(4)$ & $1.409(7)$ & - \\
\hline $\mathrm{C} 2-\mathrm{C} 2$ & - & - & $1.396(13)$ \\
\hline $\mathrm{C} 2 \mathrm{~B}-\mathrm{C} 2 \mathrm{~B}$ & - & - & $1.418(13)$ \\
\hline $\mathrm{Gd} 1 \cdots \mathrm{Gd} 2$ & $6.477(1)$ & $6.514(1)$ & - \\
\hline Gd1 $\cdots \mathrm{Gd} 1$ & - & - & $6.567(1)$ \\
\hline Gd1B $\cdots \operatorname{Gd} 1 B$ & - & - & $6.504(1)$ \\
\hline Gd-bpym torsion ${ }^{c}$ & $1.8(3)$ & $0.6(1)$ & 1.4(1) \\
\hline $\operatorname{Gd}(\mathrm{B})-$ bpym $(\mathrm{B})$ torsion $^{c}$ & - & - & $1.8(1)$ \\
\hline
\end{tabular}

${ }^{a}$ The asymmetric unit of 4-Gd contains two discrete halves of the $\left[\left(\mathrm{Cp}^{*}{ }_{2} \mathrm{Gd}\right)_{2}\left(\mu-5,5^{\prime}-\mathrm{R} 2 \mathrm{bpym}\right)\right]^{+}$ complex, each of which sits on an inversion center.

${ }^{b}$ The standard deviation of the average value was estimated from $\sigma=\sqrt{\sum \sigma_{i}^{2}} / N$, where $\sigma_{\mathrm{i}}$ is the standard deviation of each bond distance $i$ and $N$ is the number of distances averaged.

${ }^{c}$ The torsion angle between the plane of the bpym ligand and the Gd $\cdots \mathrm{Gd}$ axis 
Table S7. Selected bond distances $(\AA)$ and angles $\left({ }^{\circ}\right)$ for 1-Dy, 2-Dy, 3-Dy, and 4-Dy.

\begin{tabular}{|c|c|c|c|c|}
\hline & 1-Dy ${ }^{a}$ & 2-Dy & 3-Dy ${ }^{a}$ & 4-Dy ${ }^{a}$ \\
\hline Dy1-N1 & $2.430(8)$ & $2.418(4)$ & $2.433(6)$ & $2.436(6)$ \\
\hline Dy1-N1' & - & $2.447(4)$ & - & - \\
\hline Dy2-N3 & $2.429(8)$ & $2.448(4)$ & $2.428(6)$ & $2.456(6)$ \\
\hline Dy2-N3' & - & $2.421(5)$ & - & - \\
\hline Dy1B-N1B & $2.430(7)$ & - & $2.423(6)$ & $2.418(6)$ \\
\hline Dy1B-N3B & $2.433(9)$ & - & $2.411(6)$ & $2.450(6)$ \\
\hline Dy-N(avg) & $2.431(4)^{b}$ & $2.434(2)^{b}$ & $2.424(3)^{b}$ & $2.440(3)^{b}$ \\
\hline $\mathrm{C} 2-\mathrm{C} 2$ & - & $1.415(7)$ & - & - \\
\hline $\mathrm{C} 2-\mathrm{C} 2$ & $1.426(17)$ & - & $1.406(13)$ & $1.416(13)$ \\
\hline $\mathrm{C} 2 \mathrm{~B}-\mathrm{C} 2 \mathrm{~B}$ & $1.432(18)$ & - & $1.403(14)$ & $1.414(13)$ \\
\hline Dy $1 \cdots$ Dy 2 & - & $6.431(1)$ & - & 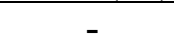 \\
\hline 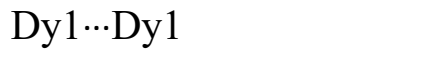 & $6.409(1)$ & - & $6.415(1)$ & $6.493(1)$ \\
\hline 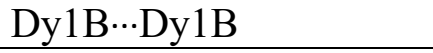 & $6.407(1)$ & - & $6.388(1)$ & $6.439(1)$ \\
\hline Dy-bpym torsion ${ }^{c}$ & $2.6(1)$ & $3.3(8)$ & $4.5(1)$ & $3.2(1)$ \\
\hline Dy $(\mathrm{B})-$ bpym $(\mathrm{B})$ torsion $^{c}$ & $1.4(1)$ & - & $3.2(1)$ & $3.5(1)$ \\
\hline
\end{tabular}

${ }^{a}$ The asymmetric unit of 3-Dy and 4-Dy contains two discrete halves of the $\left[\left(\mathrm{Cp}_{2}{ }_{2} \mathrm{Dy}\right)_{2}\left(\mu-5,5^{\prime}-\right.\right.$ R2bpym) $]^{+}$complex, each of which sits on an inversion center.

${ }^{b}$ The standard deviation of the average value was estimated from $\sigma=\sqrt{\sum \sigma_{i}^{2}} / N$, where $\sigma_{\mathrm{i}}$ is the standard deviation of each bond distance $i$ and $N$ is the number of distances averaged.

${ }^{c}$ The torsion angle between the plane of the bpym ligand and the Dy $\cdots$ Dy axis

\section{Section S5. Magnetic measurements}

\section{Section S5.1 De magnetic susceptibility data}

Dc magnetic susceptibility data were collected at temperatures ranging from 2 to $300 \mathrm{~K}$ under applied fields of 1,5 , and $10 \mathrm{kOe}$ for 1-4. The spin-only Hamiltonian $\hat{H}=-2 J_{\mathrm{Gd}-\mathrm{rad}} \hat{S}_{\mathrm{rad}} \cdot\left(\hat{S}_{\mathrm{Gd}(1)}+\right.$ $\left.\hat{S}_{\mathrm{Gd}(2)}\right)$ was used to fit the data for 3-Gd and 4-Gd, where $J_{\mathrm{Gd}-\text { rad }}$ represents the magnetic exchange coupling between each $\mathrm{Gd}^{3+}$ ion and the organic radical spin. This equation underestimated the rise in $\chi_{\mathrm{M}} T$ at low temperatures for 1-Gd and 2-Gd, and improved fits to the data could be obtained using the Hamiltonian $\hat{H}=-2 J_{\mathrm{Gd}-\mathrm{rad}} \hat{S}_{\mathrm{rad}} \cdot\left(\hat{S}_{\mathrm{Gd}(1)}+\hat{S}_{\mathrm{Gd}(2))}-2 J_{\mathrm{Gd}-\mathrm{Gd}} \hat{S}_{\mathrm{Gd}(1)} \cdot \hat{S}_{\mathrm{Gd}(2)}\right.$, where $J_{\mathrm{Gd}-\mathrm{Gd}}$ represents the magnetic exchange coupling between $\mathrm{Gd}^{3+}$ ions. Fits to the data for 3-Gd and 4-Gd using the latter Hamiltonian yielded $J_{\mathrm{Gd}-\mathrm{Gd}}<1 \times 10^{-4} \mathrm{~cm}^{-1}$ and comparable values of $J_{\mathrm{Gd}-\text { rad }}$ to those obtained using the former Hamiltonian, which considers only intramolecular $\mathrm{Gd}^{3+}-\mathrm{bpym}^{\bullet-}$ coupling. Contributions for temperature-independent paramagnetism (TIP) and intermolecular coupling $\left(z J^{\prime}\right)$ were included when fitting all susceptibility data for 1-Gd through 4-Gd. 


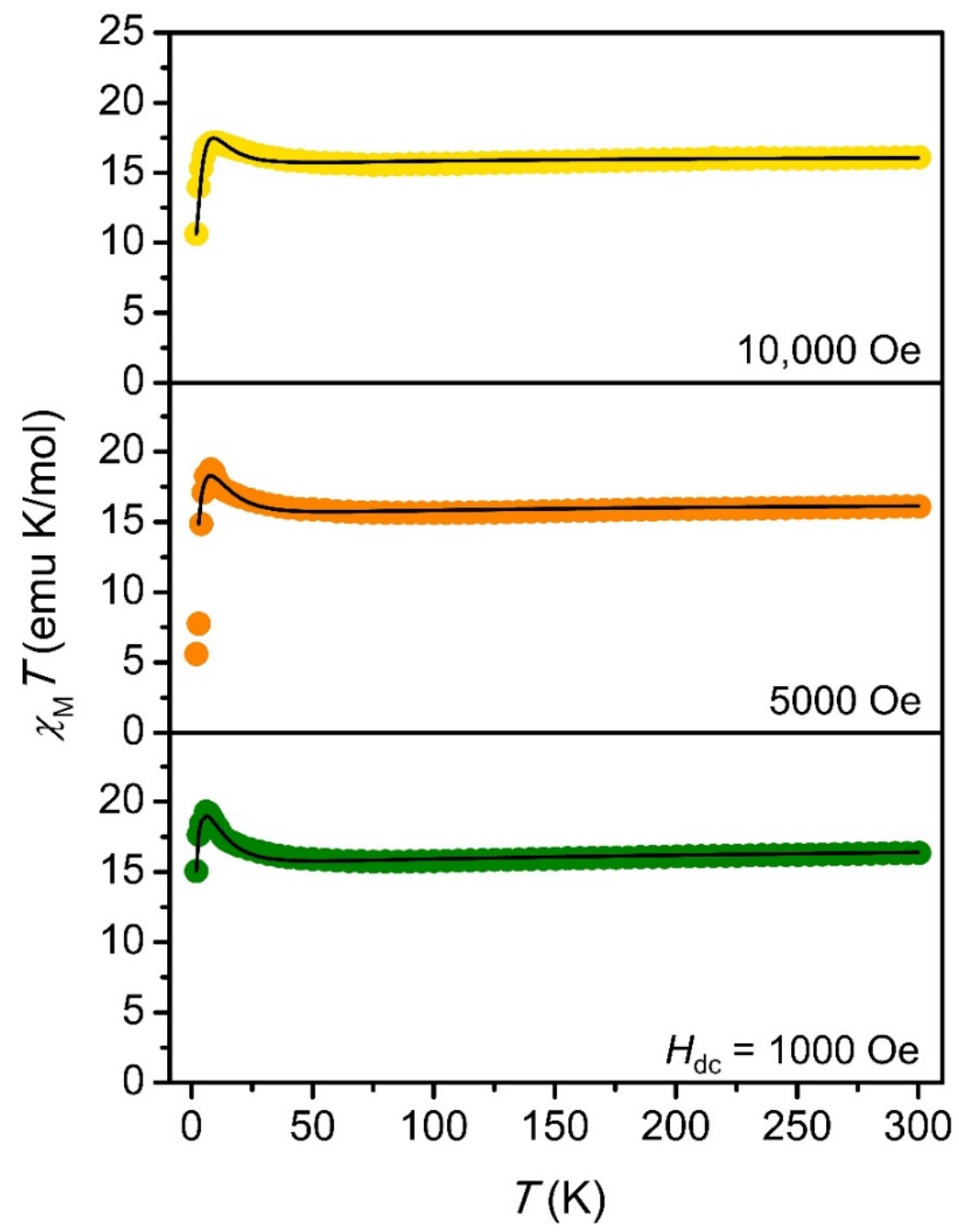

Figure S40. Zero-field cooled dc magnetic susceptibility data for 1-Gd under fields of 1, 5, and $10 \mathrm{kOe}$. Black lines represent fits to the data as described above.

Table S8. Parameters used to fit dc magnetic susceptibility data for 1-Gd and magnetic exchange coupling constants $(J)$ extracted from those fits.

\begin{tabular}{ccccc}
\hline Field $(\mathbf{O e})$ & $\boldsymbol{J}_{\mathbf{G d - r a d}}\left(\mathbf{c m}^{-\mathbf{1}}\right)$ & $\boldsymbol{J}_{\mathbf{G d}-\mathbf{G d}}\left(\mathbf{c m}^{-\mathbf{1}}\right)$ & $\mathbf{z} \boldsymbol{J}^{\mathbf{}}\left(\mathbf{c m}^{-\mathbf{1}}\right)$ & $\mathbf{T I P}\left(\mathbf{c m}^{\mathbf{3}} \mathbf{m o l}^{\mathbf{- 1}}\right)$ \\
\hline 1000 & $-2.88(6)$ & $0.122(5)$ & $-0.0108(2)$ & $0.00131(8)$ \\
5000 & $-3.10(11)$ & $0.137(8)$ & $-0.0138(4)$ & $0.000517(2)$ \\
10,000 & $-2.66(12)$ & $0.125(8)$ & $-0.0118(2)$ & $0.000203(3)$ \\
\hline
\end{tabular}




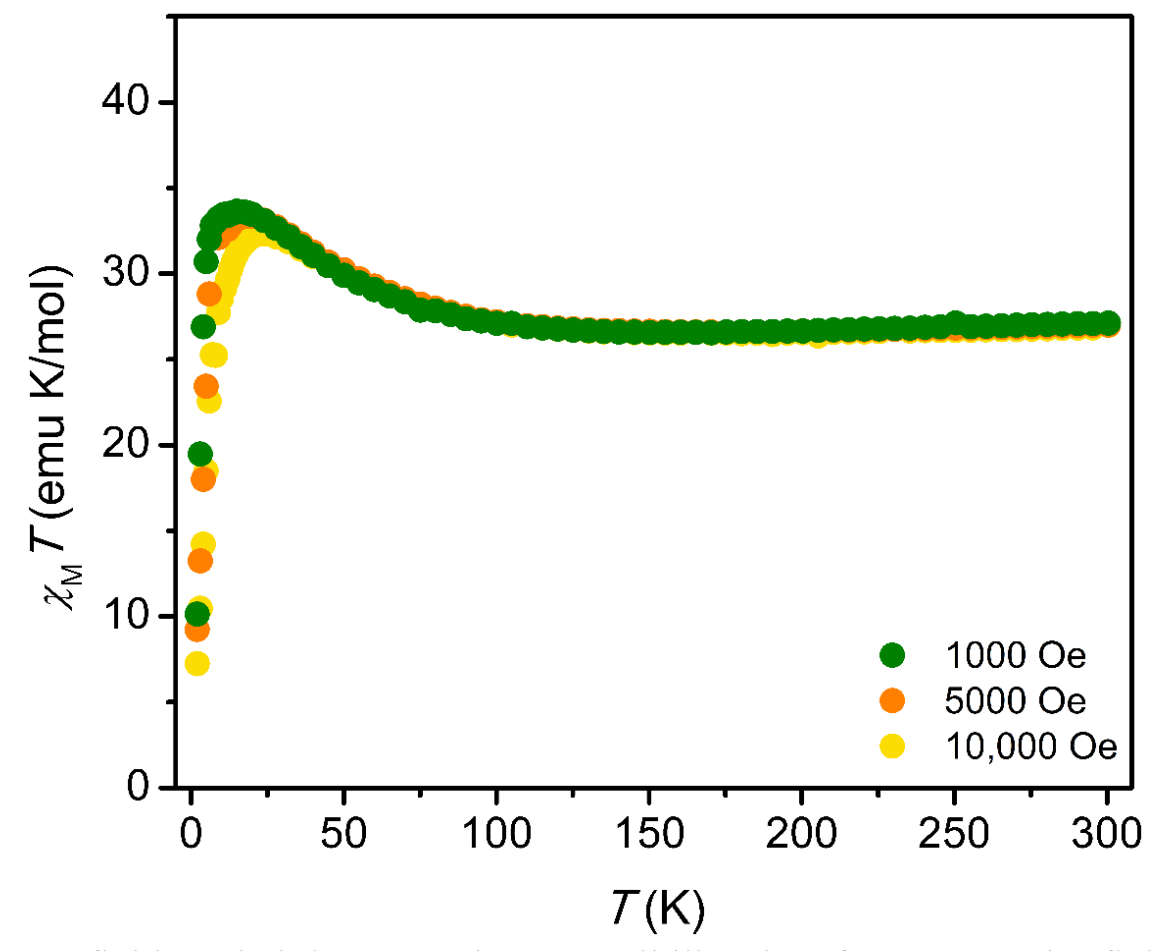

Figure S41. Zero-field cooled dc magnetic susceptibility data for 1-Dy under fields of 1, 5, and $10 \mathrm{kOe}$.

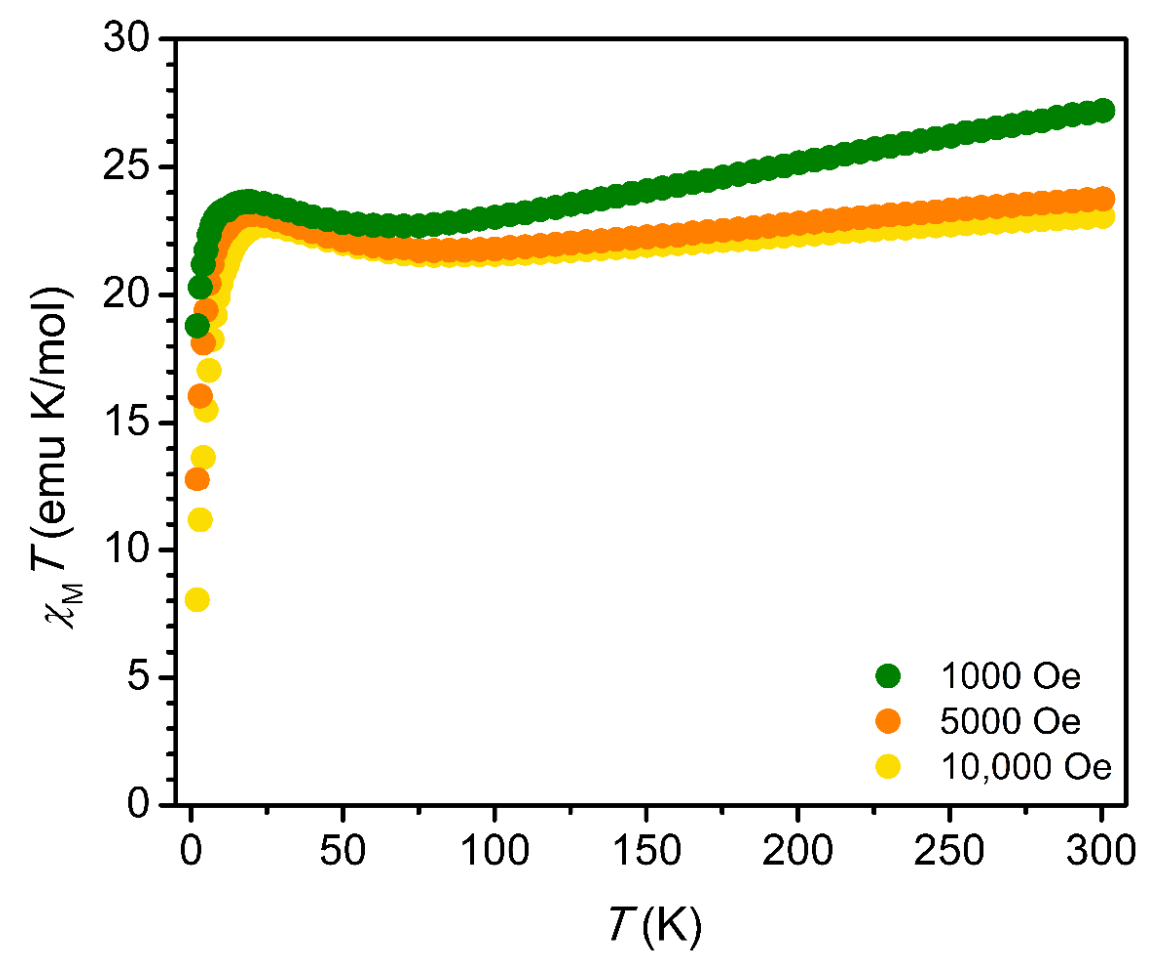

Figure S42. Zero-field cooled dc magnetic susceptibility data for 1-Tb under fields of 1, 5, and $10 \mathrm{kOe}$. 


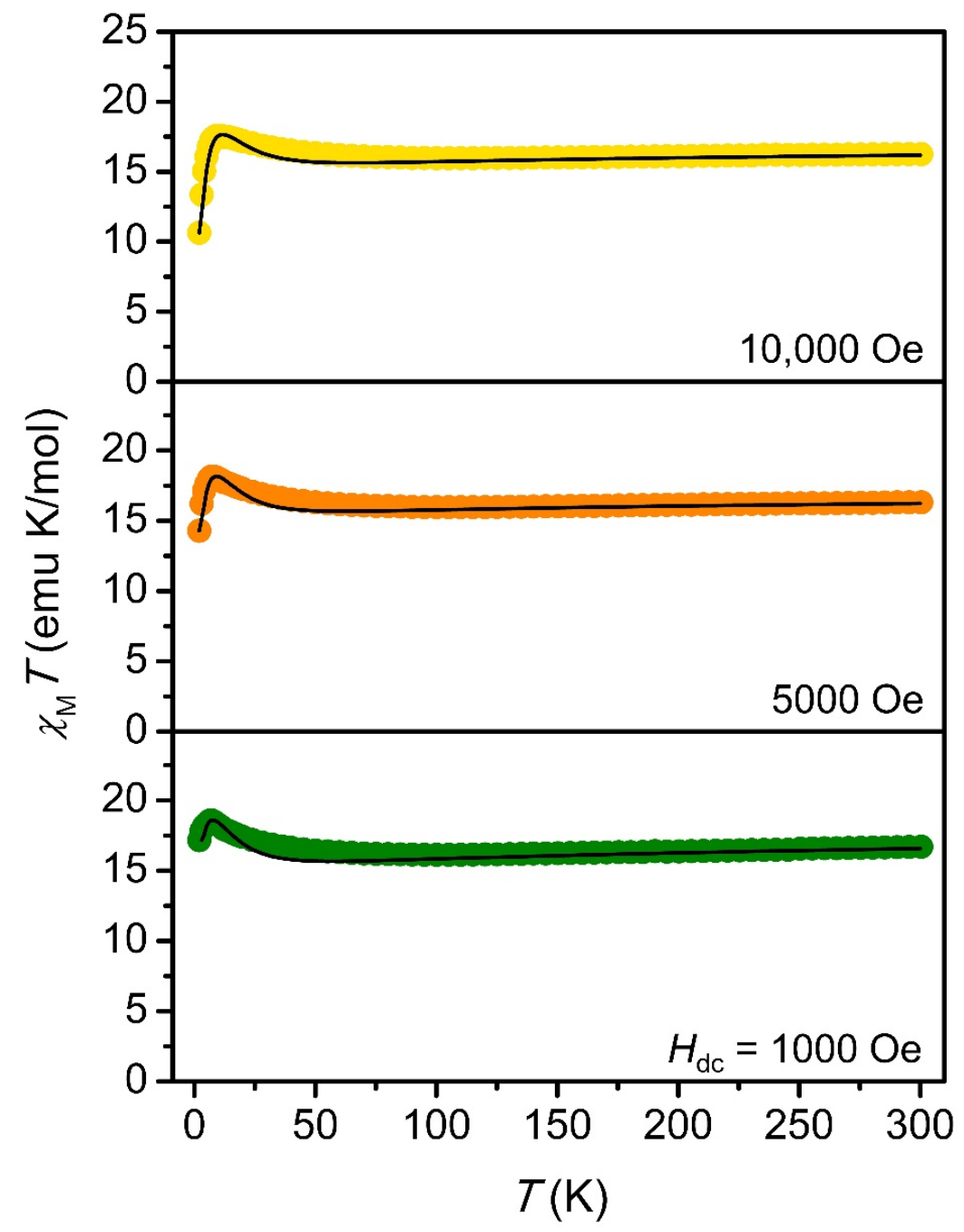

Figure S43. Zero-field cooled dc magnetic susceptibility data for 2-Gd under fields of 1, 5, and $10 \mathrm{kOe}$. Black lines represent fits to the data used to extract the magnetic coupling constant $(J)$.

Table S9. Parameters used to fit dc magnetic susceptibility data for $\mathbf{2}-\mathbf{G d}$ and magnetic exchange coupling constants $(J)$ extracted from those fits.

\begin{tabular}{ccccc}
\hline Field (Oe) & $\boldsymbol{J}_{\mathbf{G d}-\text { rad }}\left(\mathbf{c m}^{-\mathbf{1}}\right)$ & $\boldsymbol{J}_{\mathbf{G d}-\mathbf{G d}}\left(\mathbf{c m}^{-\mathbf{1}}\right)$ & $\mathbf{Z} \boldsymbol{J}\left(\mathbf{c m}^{-\mathbf{1}}\right)$ & $\mathbf{T I P}\left(\mathbf{c m}^{\mathbf{3}} \mathbf{m o l}^{-\mathbf{1}}\right)$ \\
\hline 1000 & $-3.81(16)$ & $0.108(4)$ & $-0.0149(9)$ & $0.00227(2)$ \\
5000 & $-3.76(15)$ & $0.141(4)$ & $-0.0171(7)$ & $0.00103(1)$ \\
10,000 & $-4.16(25)$ & $0.145(5)$ & $-0.0193(14)$ & $0.000956(2)$ \\
\hline
\end{tabular}




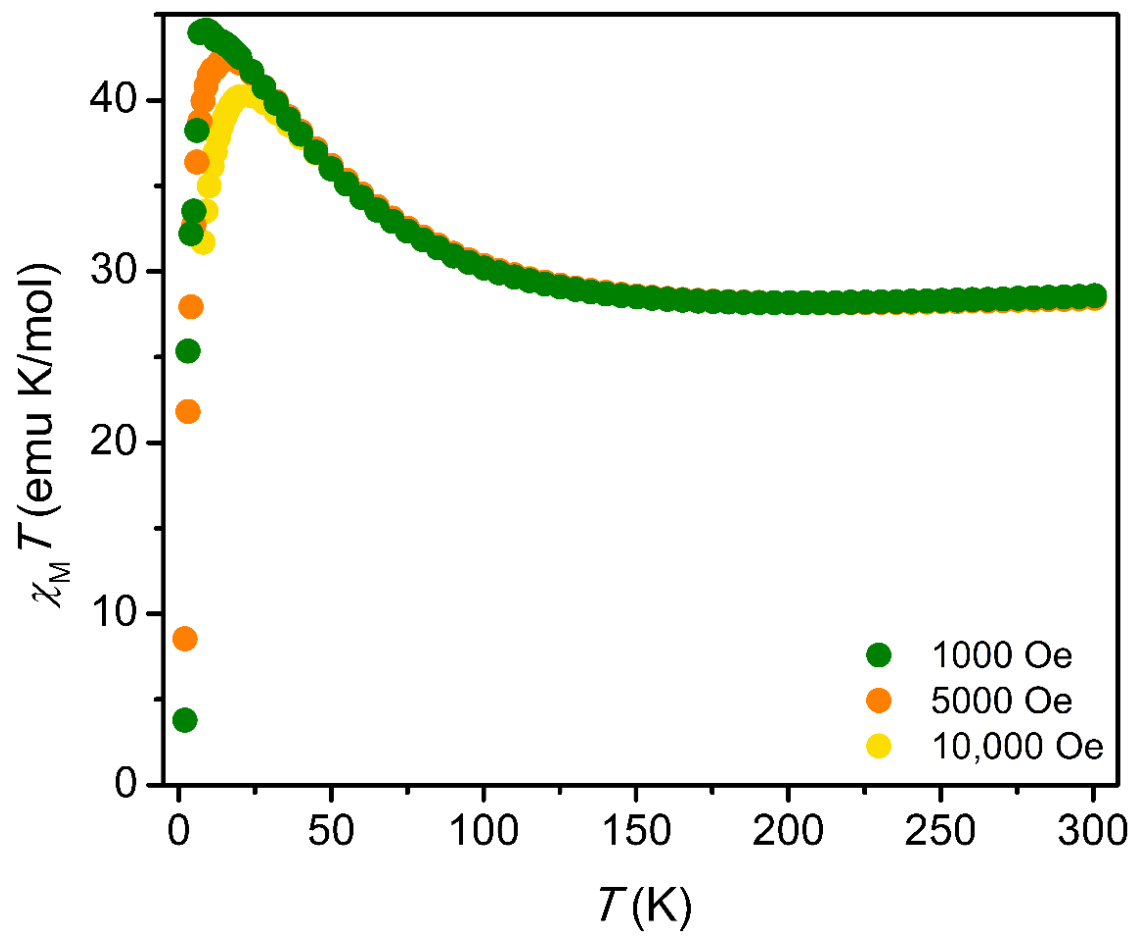

Figure S44. Zero-field cooled dc magnetic susceptibility data for 2-Dy under applied fields of 1, 5 , and $10 \mathrm{kOe}$. 


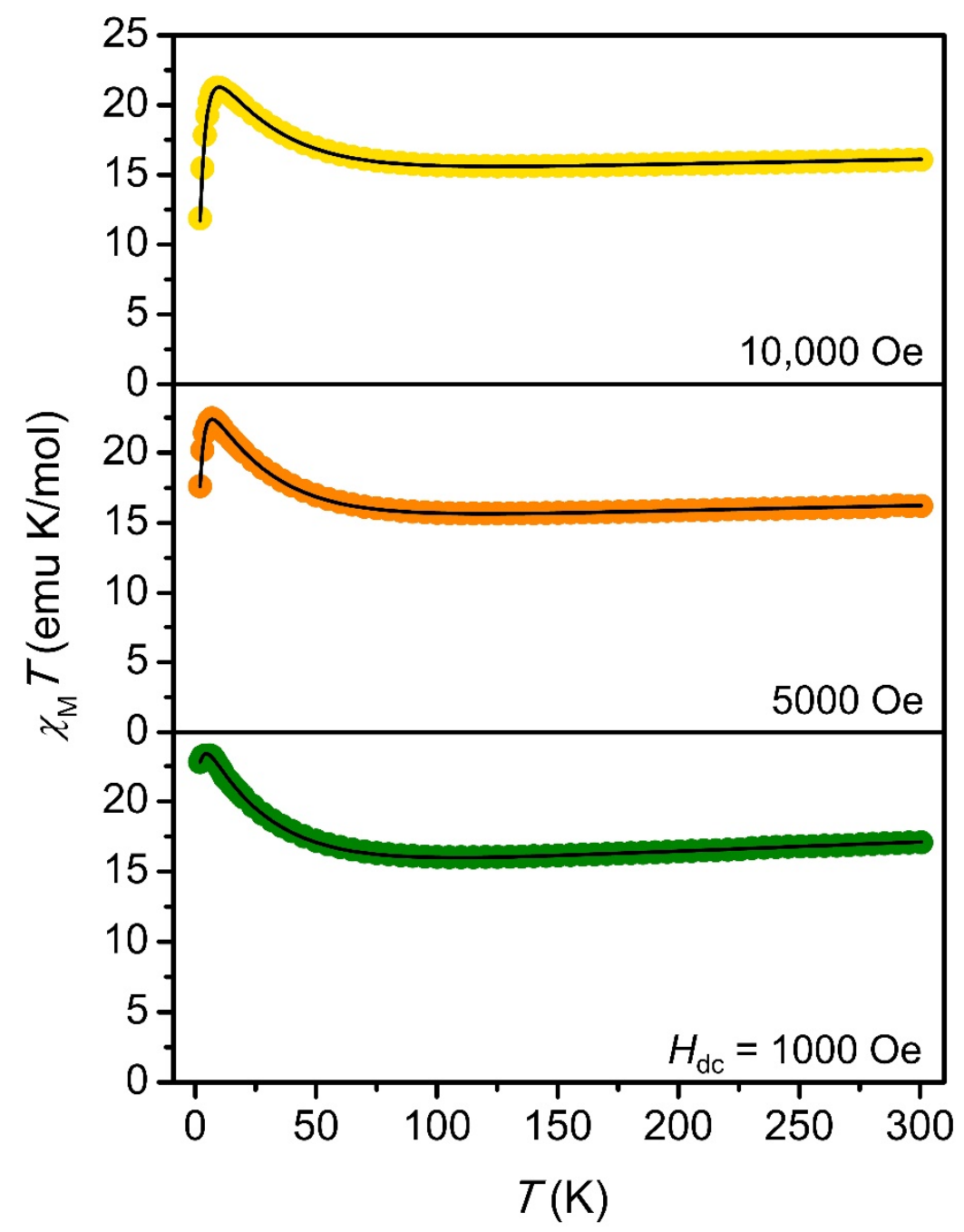

Figure S45. Zero-field cooled dc magnetic susceptibility data for 3-Gd under fields of 1, 5, and $10 \mathrm{kOe}$. Black lines represent fits to the data used to extract the magnetic coupling constant $(J)$.

Table S10. Parameters used to fit dc magnetic susceptibility data for 3-Gd and magnetic exchange coupling constants $(J)$ extracted from those fits.

\begin{tabular}{cccc}
\hline Field (Oe) & $\boldsymbol{J}\left(\mathbf{c m}^{-\mathbf{1}}\right)$ & $\mathbf{z} \boldsymbol{J}\left(\mathbf{c m}^{-\mathbf{1}}\right)$ & TIP $\left(\mathbf{c m}^{\mathbf{3}} \mathbf{m o l}^{-\mathbf{1}}\right)$ \\
\hline 1000 & $-9.63(8)$ & $-0.00142(1)$ & $0.00512(10)$ \\
5000 & $-9.42(8)$ & $-0.00170(1)$ & $0.00220(9)$ \\
10,000 & $-9.54(7)$ & $-0.00168(1)$ & $0.00175(8)$ \\
\hline
\end{tabular}




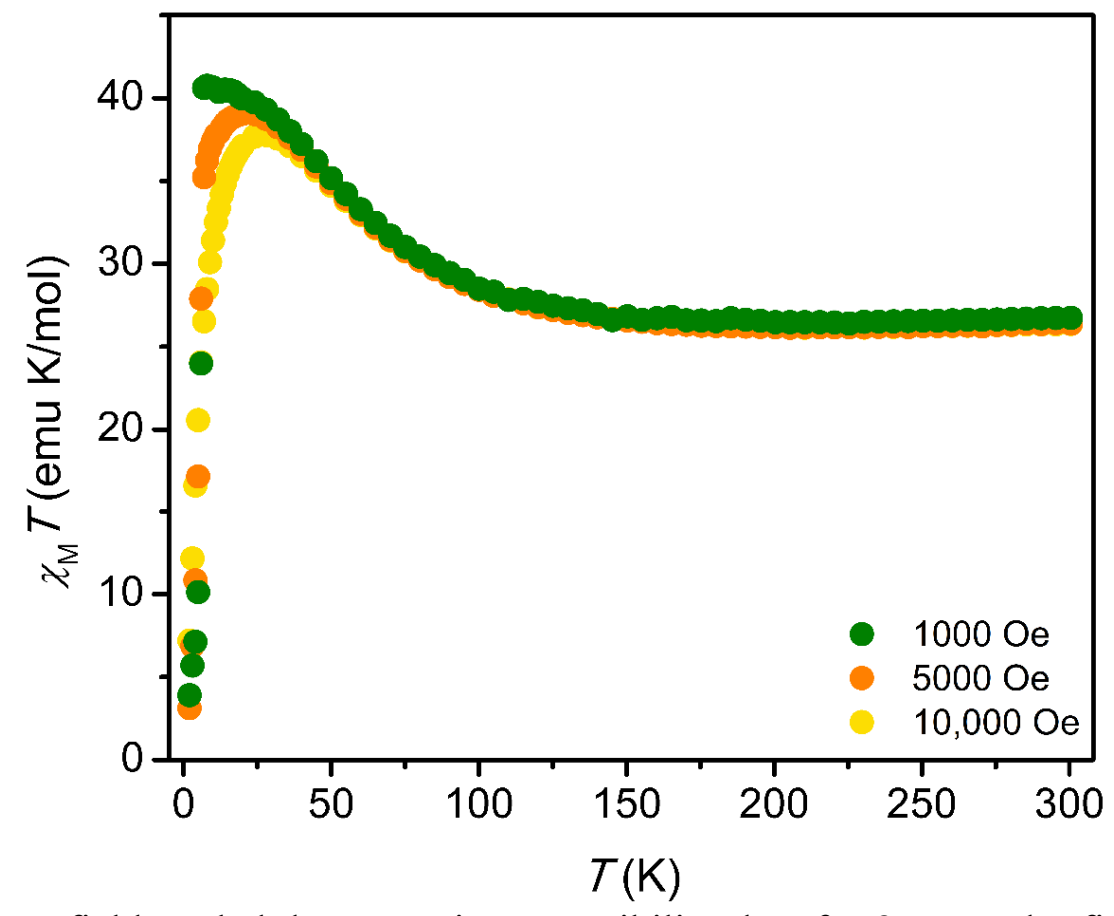

Figure S46. Zero-field cooled dc magnetic susceptibility data for 3-Dy under fields of 1, 5, and $10 \mathrm{kOe}$. 


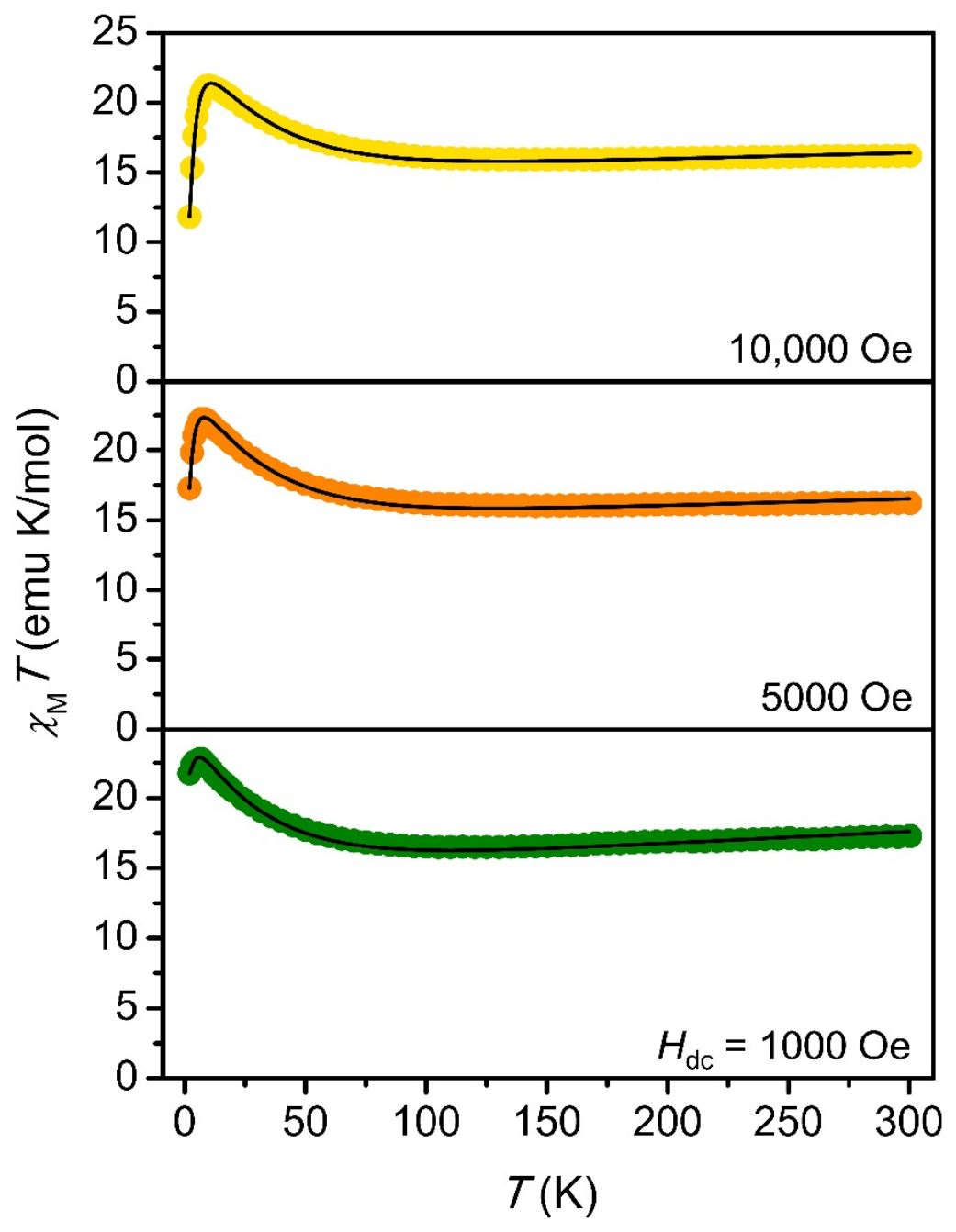

Figure S47. Zero-field cooled de magnetic susceptibility data for 4-Gd under fields of 1, 5, and $10 \mathrm{kOe}$. Black lines represent fits to the data used to extract the magnetic coupling constant $(J)$.

Table S11. Parameters used to fit dc magnetic susceptibility data for 4-Gd and magnetic exchange coupling constants $(J)$ extracted from those fits.

\begin{tabular}{cccc}
\hline Field (Oe) & $\boldsymbol{J}\left(\mathbf{c m}^{-\mathbf{1}}\right)$ & $\left.\mathbf{z} \boldsymbol{J}^{\mathbf{(}} \mathbf{c m}^{-\mathbf{1}}\right)$ & TIP $\left(\mathbf{c m}^{\mathbf{3}} \mathbf{m o l}^{\mathbf{- 1}}\right)$ \\
\hline 1000 & $-10.8(3)$ & $-0.00297(3)$ & $0.00694(3)$ \\
5000 & $-11.0(3)$ & $-0.00294(3)$ & $0.00335(2)$ \\
10,000 & $-11.1(2)$ & $-0.00310(4)$ & $0.00299(2)$ \\
\hline
\end{tabular}




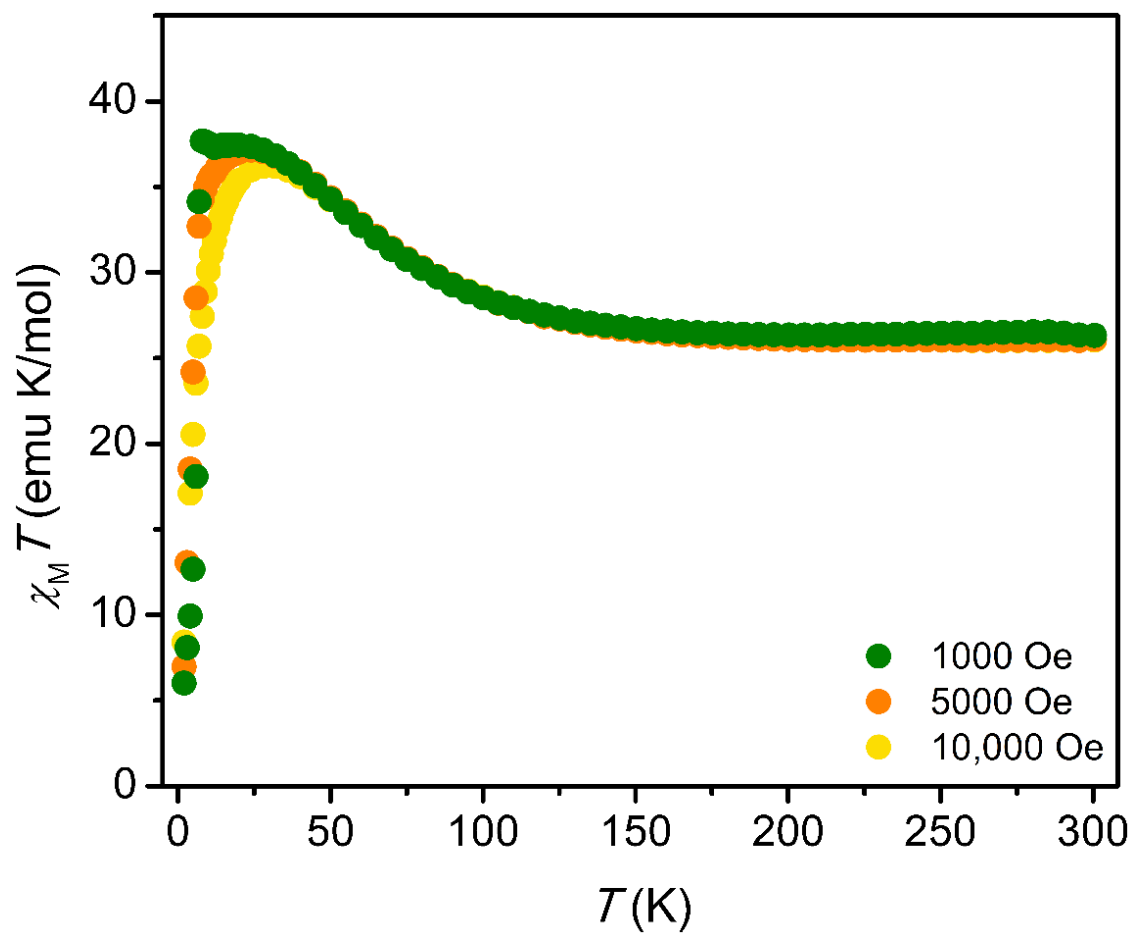

Figure S48. Zero-field cooled dc magnetic susceptibility data for 4-Dy under fields of 1, 5, and $10 \mathrm{kOe}$.

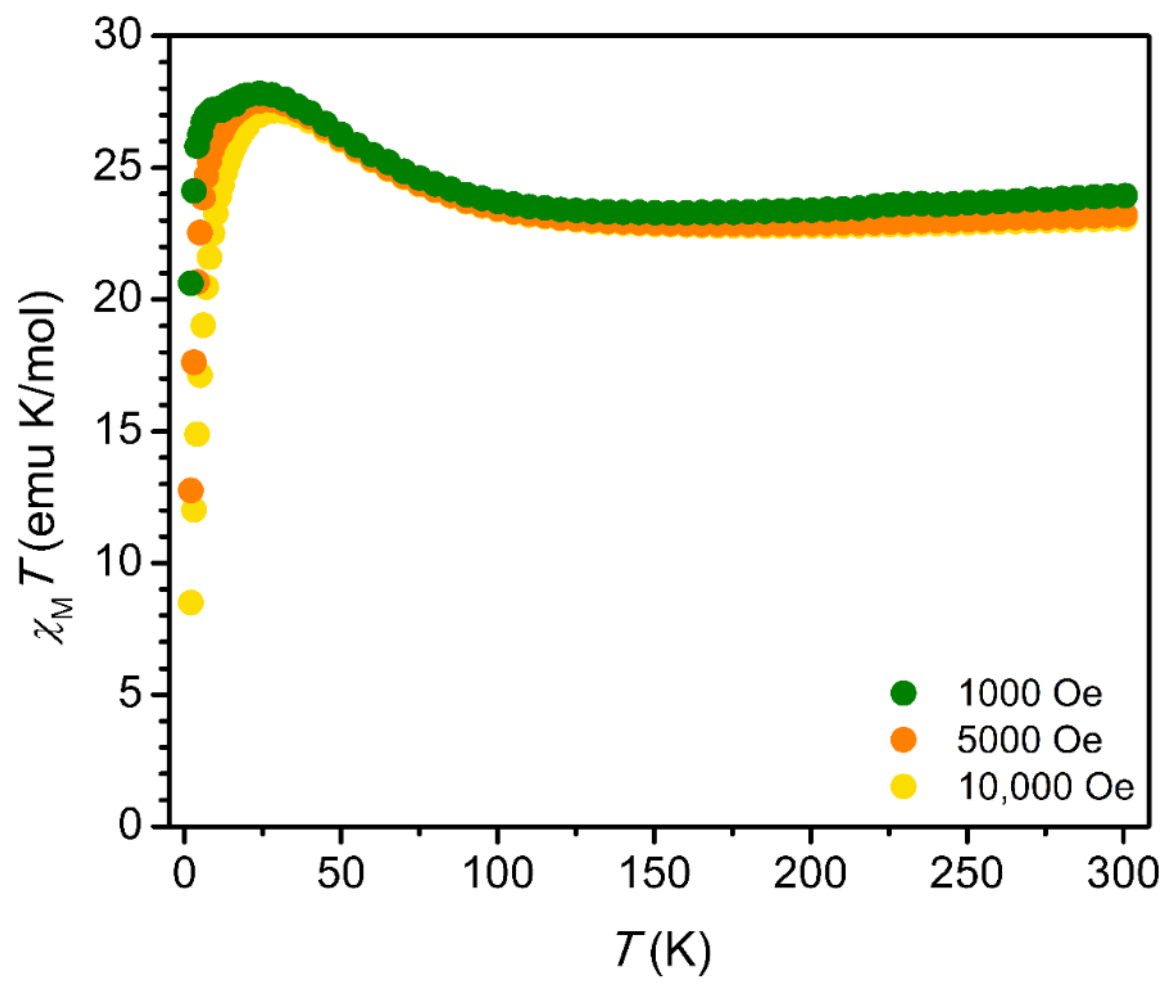

Figure S49. Zero-field cooled dc magnetic susceptibility data for 4-Tb under fields of 1, 5, and $10 \mathrm{kOe}$. 


\section{Section S5.2 Ac magnetic susceptibility data}

Ac magnetic susceptibility measurements were performed with a probe field of $4 \mathrm{Oe}$ at frequencies ranging from 0.1 to $1500 \mathrm{~Hz}$ for 1-Dy, 1-Tb, 2-Dy, 3-Dy, 4-Dy, and 4-Tb. Magnetic relaxation times, $\tau$, were extracted from a simultaneous fit of in-phase $\left(\chi \mathrm{m}^{\prime}\right)$ and out-of-phase $\left(\chi \mathrm{m}^{\prime \prime}\right)$ components of the magnetic susceptibility to a generalized Debye model. The $\alpha$ values extracted from fits to $\chi \mathrm{M}^{\prime}$ and $\chi \mathrm{M}^{\prime \prime}$ data were used to calculate uncertainty ranges for $\tau$ according to the following equation:

$$
\tau_{ \pm}(1 \sigma)=\tau_{\mu} e^{ \pm \frac{1.82 \sqrt{\alpha}}{1-\alpha}}
$$

where $\tau_{ \pm}(1 \sigma)$ is the uncertainty range for one standard deviation from the mean and $\tau_{\mu}$ is the mean relaxation time. ${ }^{1}$ It is important to emphasize that the value of $\tau_{ \pm}$is not indicative of experimental error. Fits of $\chi \mathrm{M}^{\prime}$ and $\chi_{\mathrm{M}}{ }^{\prime \prime}$ data to a generalized Debye model show low residuals for all compounds measured. Large $\tau_{ \pm}$values instead arise from large $\alpha$ values, which indicate a wide distribution of relaxation times. Large $\alpha$ values are an intrinsic property of a compound, and they may be related to crystallographic disorder or electronic structure. ${ }^{1}$ Indeed, the large $\alpha$ values for 1-Dy are reproduced across several measured samples using the same measurement parameters utilized for other compounds.

For 1-Dy and 2-Dy, two relaxation processes could be distinguished as two separate peaks in the out-of-phase susceptibility data. For 1-Dy, a fast process could be distinguished at low temperatures $(4.5$ to $5.5 \mathrm{~K})$ and a slow process persisted at higher temperatures $(9$ to $11 \mathrm{~K}) \cdot \chi_{\mathrm{M}}$ and $\chi \mathrm{M}^{\prime \prime}$ data corresponding to each peak were fit separately to a generalized Debye model in these temperature regimes to extract relaxation times for each process. The two relaxation processes overlapped significantly at intermediate temperatures ( 6 to $8.5 \mathrm{~K}$ ), but the $\chi \mathrm{m}^{\prime}$ and $\chi \mathrm{M}^{\prime \prime}$ data at these temperatures could be fit using a generalized Debye model that accounted for both processes. The $\tau$ values in this model were restrained with upper and lower bounds based upon the relaxation times extracted from fits to the high and low temperature data. It was challenging to obtain a unique fit to the data without restraining the model due to the large number of parameters. For 2-Dy, the two relaxation processes could be distinguished across a large temperature range. The fast process was observed from 5 to $10 \mathrm{~K}$ and the slow process was observed from 8 to $13 \mathrm{~K} \cdot \chi_{\mathrm{M}}$ and $\chi \mathrm{M}^{\prime \prime}$ data corresponding to each peak were fit separately to a generalized Debye model to extract relaxation times for each process.

For 1-Tb, one relaxation process was apparent as a full peak in $\chi \mathrm{M}^{\prime \prime}$ within the frequency range of the magnetometer, while a second other process was visible as tails at higher frequencies. Fit data were therefore only extracted for the lower frequency process. 


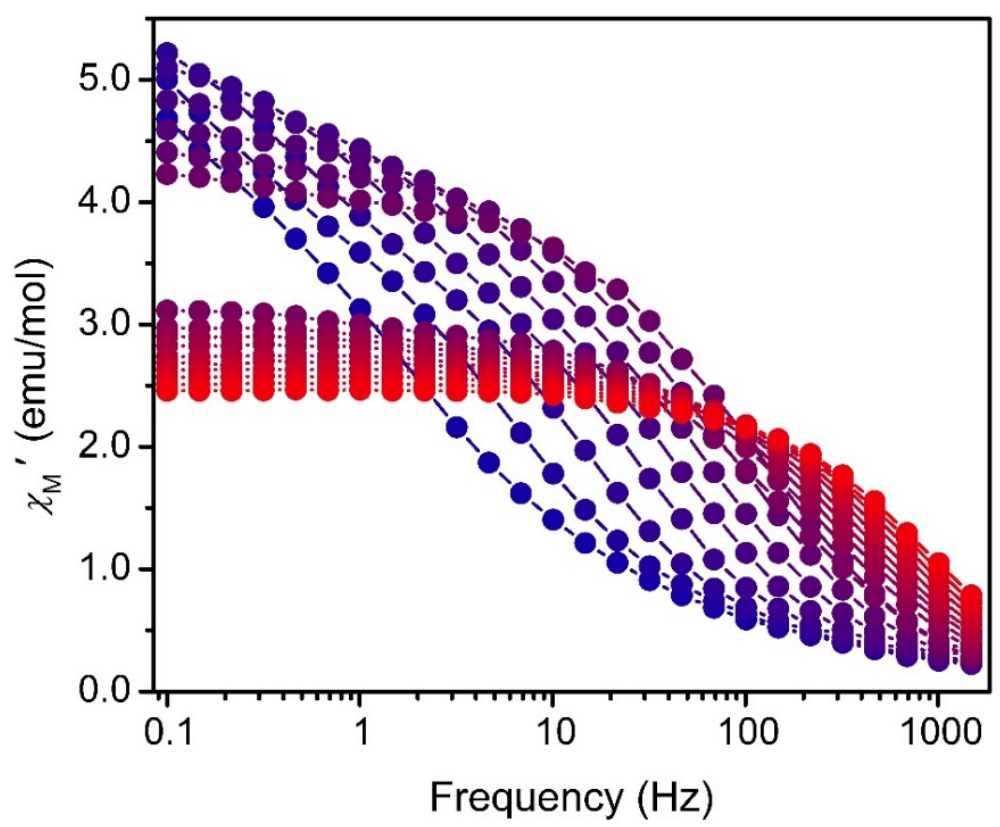

- $4.50 \mathrm{~K}$

- $5.00 \mathrm{~K}$

- $5.50 \mathrm{~K}$

- $6.00 \mathrm{~K}$

- $6.50 \mathrm{~K}$

- $7.00 \mathrm{~K}$

- $7.50 \mathrm{~K}$

- $8.00 \mathrm{~K}$

- $8.50 \mathrm{~K}$

- $9.00 \mathrm{~K}$

- $9.25 \mathrm{~K}$

- $9.50 \mathrm{~K}$

- $9.75 \mathrm{~K}$

- $10.00 \mathrm{~K}$

- $10.25 \mathrm{~K}$

- $10.50 \mathrm{~K}$

- $10.75 \mathrm{~K}$

- $11.00 \mathrm{~K}$

Frequency $(\mathrm{Hz})$

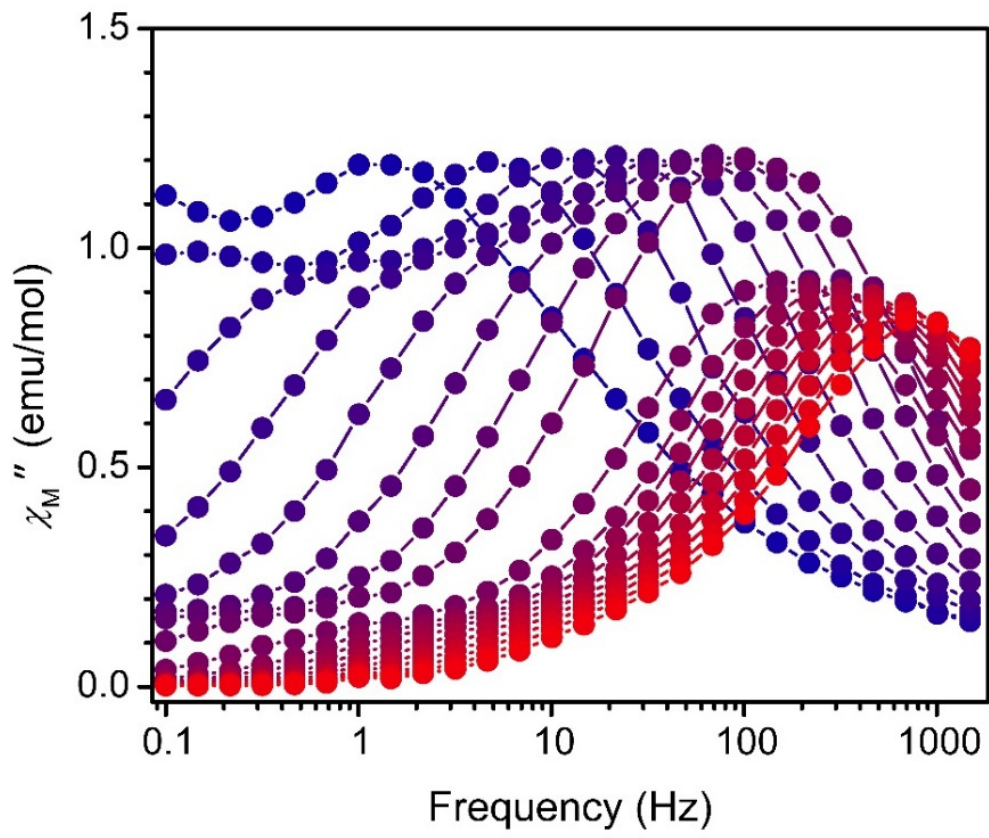

\begin{tabular}{ll} 
& $4.50 \mathrm{~K}$ \\
\hline & $5.00 \mathrm{~K}$ \\
- & $5.50 \mathrm{~K}$ \\
- & $6.00 \mathrm{~K}$ \\
- & $6.50 \mathrm{~K}$ \\
- & $7.00 \mathrm{~K}$ \\
- & $7.50 \mathrm{~K}$ \\
- & $8.00 \mathrm{~K}$ \\
- & $8.50 \mathrm{~K}$ \\
- & $9.00 \mathrm{~K}$ \\
- & $9.25 \mathrm{~K}$ \\
- & $9.50 \mathrm{~K}$ \\
- & $9.75 \mathrm{~K}$ \\
- & $10.00 \mathrm{~K}$ \\
\hline & $10.25 \mathrm{~K}$ \\
\hline & $10.50 \mathrm{~K}$ \\
\hline & $10.75 \mathrm{~K}$ \\
\hline & $11.00 \mathrm{~K}$
\end{tabular}

Figure S50. In-phase $\left(\chi_{\mathrm{M}^{\prime}}\right.$, top) and out-of-phase ( $\chi_{\mathrm{M}}{ }^{\prime \prime}$, bottom) components of the ac magnetic susceptibility for 1-Dy under zero applied dc field at frequencies ranging from $0.1-1500 \mathrm{~Hz}$ and temperatures from $4.5-9 \mathrm{~K}(0.5 \mathrm{~K}$ steps $)$ and $9-11 \mathrm{~K}(0.25 \mathrm{~K}$ steps $)$. The colored lines are guides for the eye. 


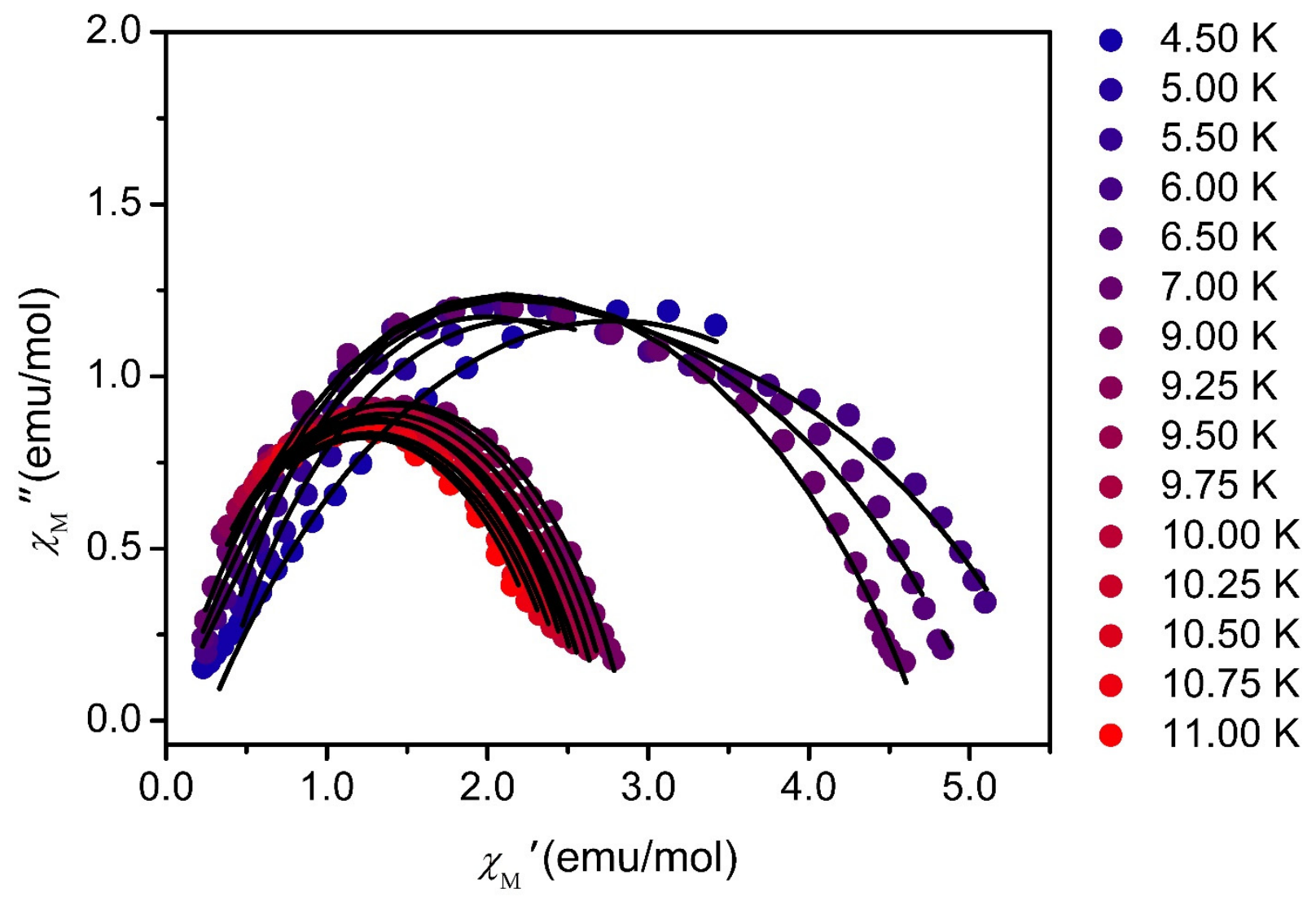

Figure S51. Cole-Cole plots for 1-Dy from 4.5-7 K (0.5 K steps) and 9-11 K (0.25 K steps). The black lines represent fits to the data using a generalized Debye model, which were used to extract $\tau$ values at each temperature. 

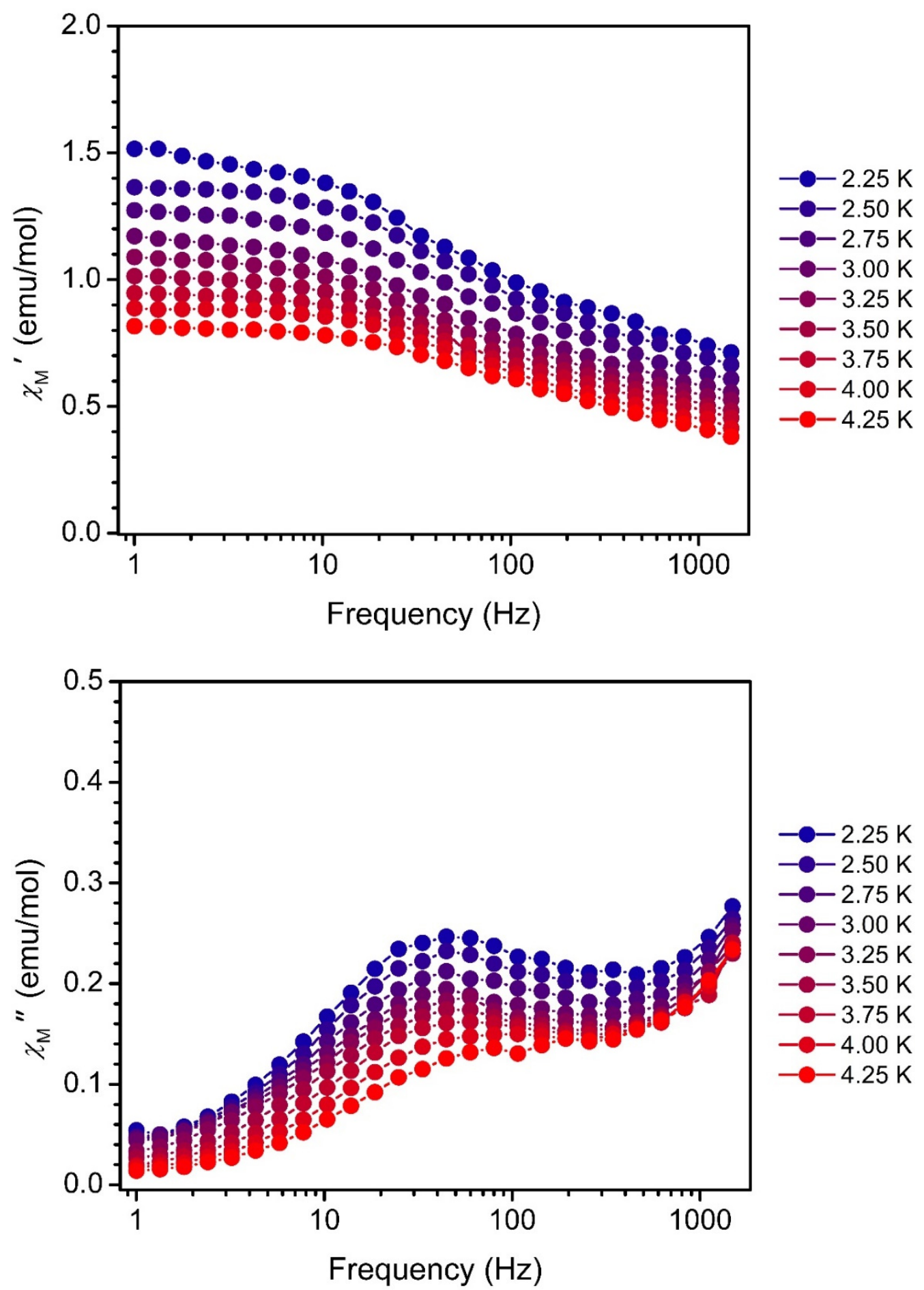

Figure S52. In-phase $\left(\chi_{\mathrm{M}^{\prime}}\right.$, top) and out-of-phase ( $\chi_{\mathrm{M}}{ }^{\prime \prime}$, bottom) components of the ac magnetic susceptibility for 1-Tb under zero applied dc field at frequencies ranging from $1-1500 \mathrm{~Hz}$ and temperatures from $2.25-4.25 \mathrm{~K}(0.25 \mathrm{~K}$ steps $)$. The colored lines are guides for the eye. 


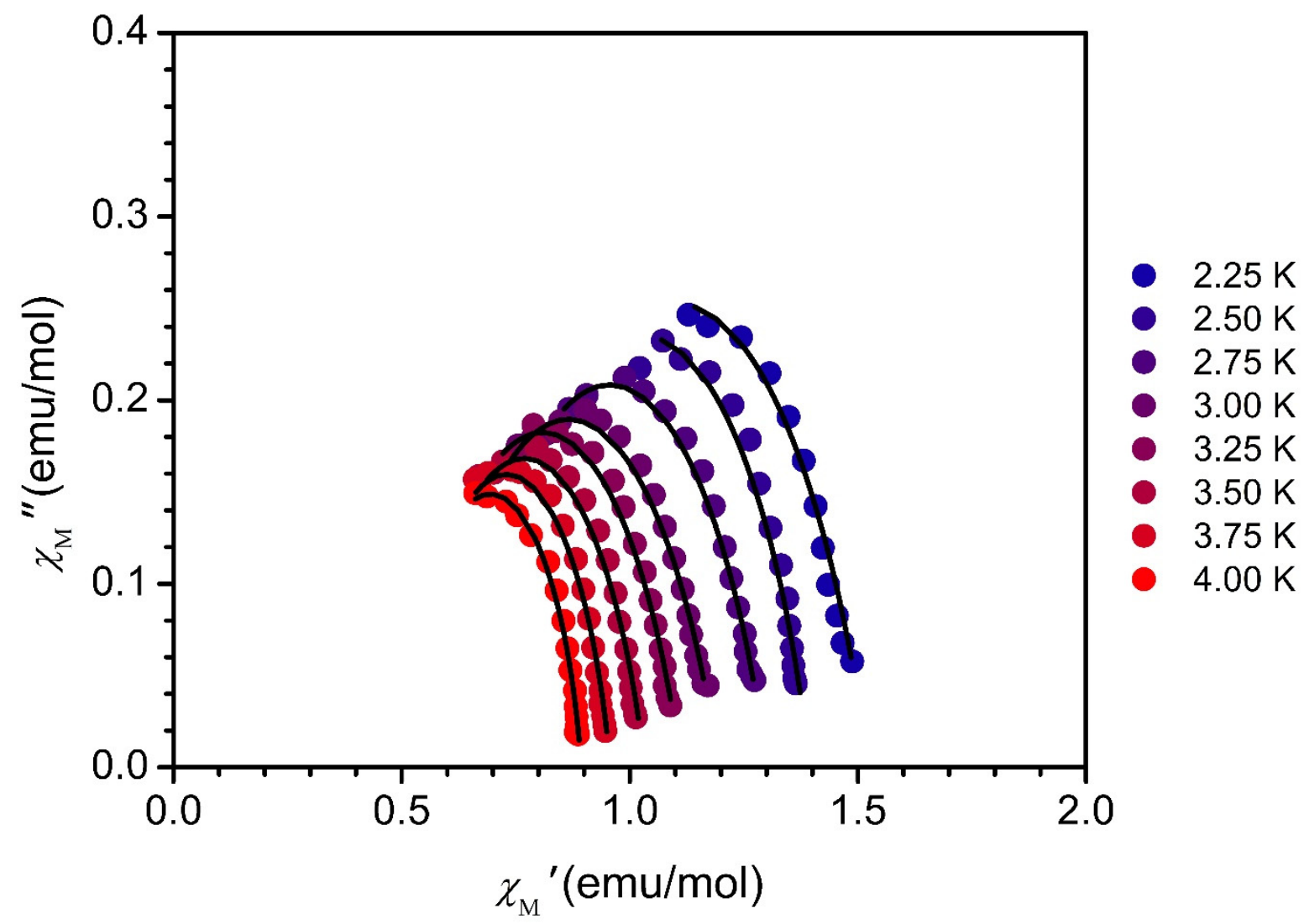

Figure S53. Cole-Cole plots for 1-Tb from 2.25-4.00 K (0.25 K steps). The black lines represent fits to the data using a generalized Debye model, which were used to extract $\tau$ values at each temperature. 


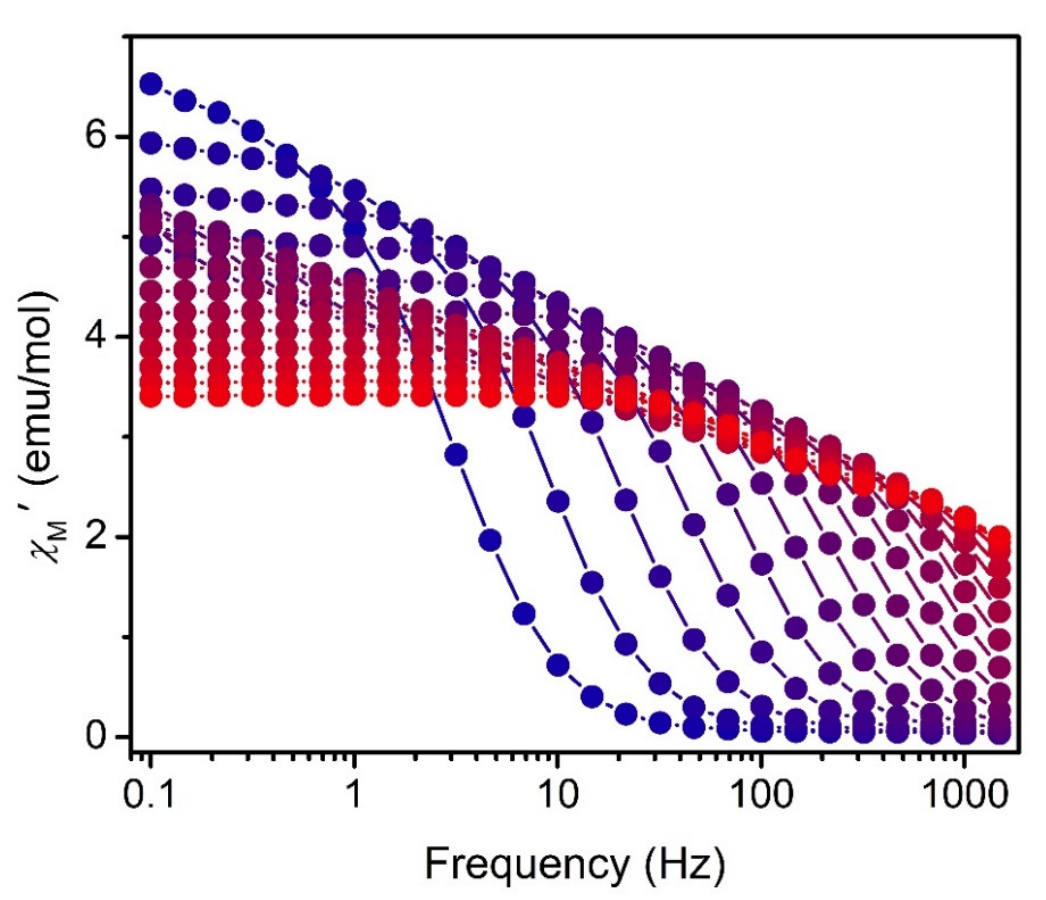

- $5 \mathrm{~K}$

- $5.5 \mathrm{~K}$

- $6 \mathrm{~K}$

- $6.5 \mathrm{~K}$

- $7 \mathrm{~K}$

- $7.5 \mathrm{~K}$

- $8 \mathrm{~K}$

- $8.5 \mathrm{~K}$

- $9 \mathrm{~K}$

- $9.5 \mathrm{~K}$

- $10 \mathrm{~K}$

- $10.5 \mathrm{~K}$

- $11 \mathrm{~K}$

- $11.5 \mathrm{~K}$

- $12 \mathrm{~K}$

- $12.5 \mathrm{~K}$

Frequency $(\mathrm{Hz})$

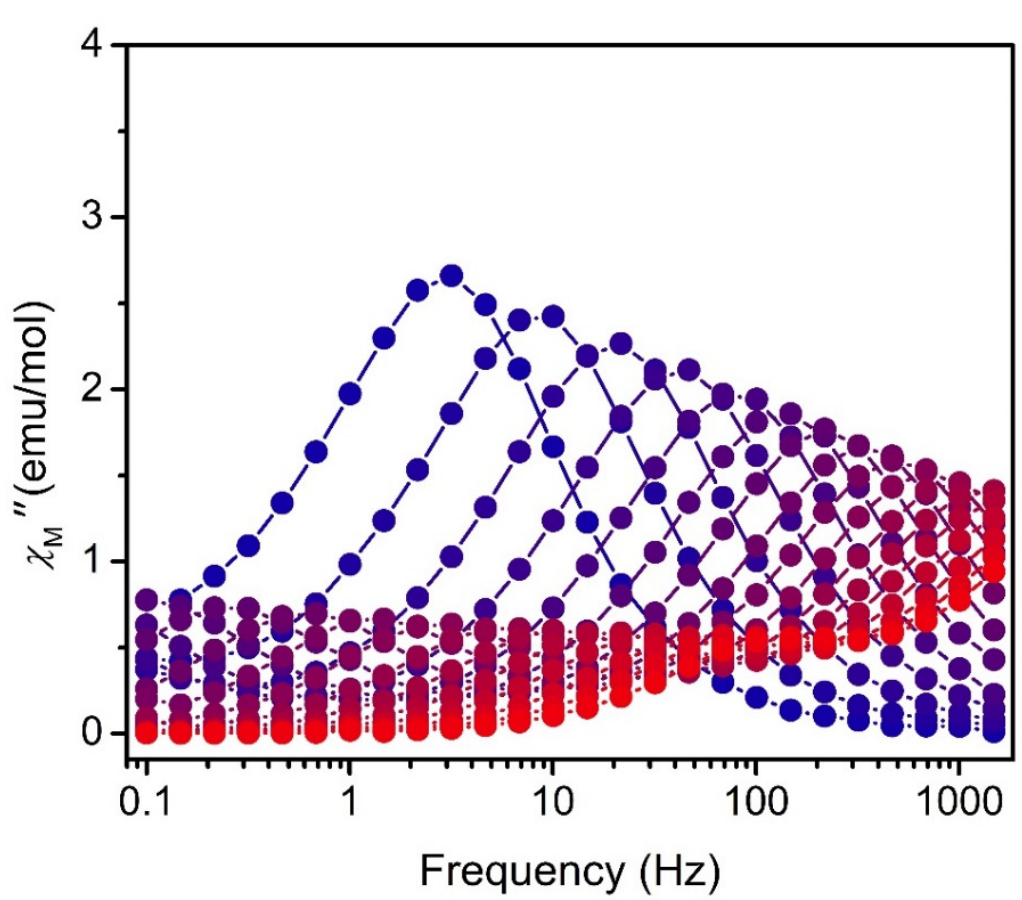

- $5 \mathrm{~K}$

- $5.5 \mathrm{~K}$

- $6 \mathrm{~K}$

- $6.5 \mathrm{~K}$

- $7 \mathrm{~K}$

- $7.5 \mathrm{~K}$

- $8 \mathrm{~K}$

- $8.5 \mathrm{~K}$

- $9 \mathrm{~K}$

- $9.5 \mathrm{~K}$

- $10 \mathrm{~K}$

- $10.5 \mathrm{~K}$

- $11 \mathrm{~K}$

- $11.5 \mathrm{~K}$

- $12 \mathrm{~K}$

- $12.5 \mathrm{~K}$

Frequency $(\mathrm{Hz})$

Figure S54. In-phase $\left(\chi_{\mathrm{M}^{\prime}}\right.$, top) and out-of-phase $\left(\chi_{\mathrm{M}}{ }^{\prime \prime}\right.$, bottom) components of the ac magnetic susceptibility for 2-Dy under zero applied dc field at frequencies ranging from $0.1-1500 \mathrm{~Hz}$ and temperatures from $5.0-12.5 \mathrm{~K}$ ( $0.5 \mathrm{~K}$ steps $)$. The colored lines are guides for the eye. 


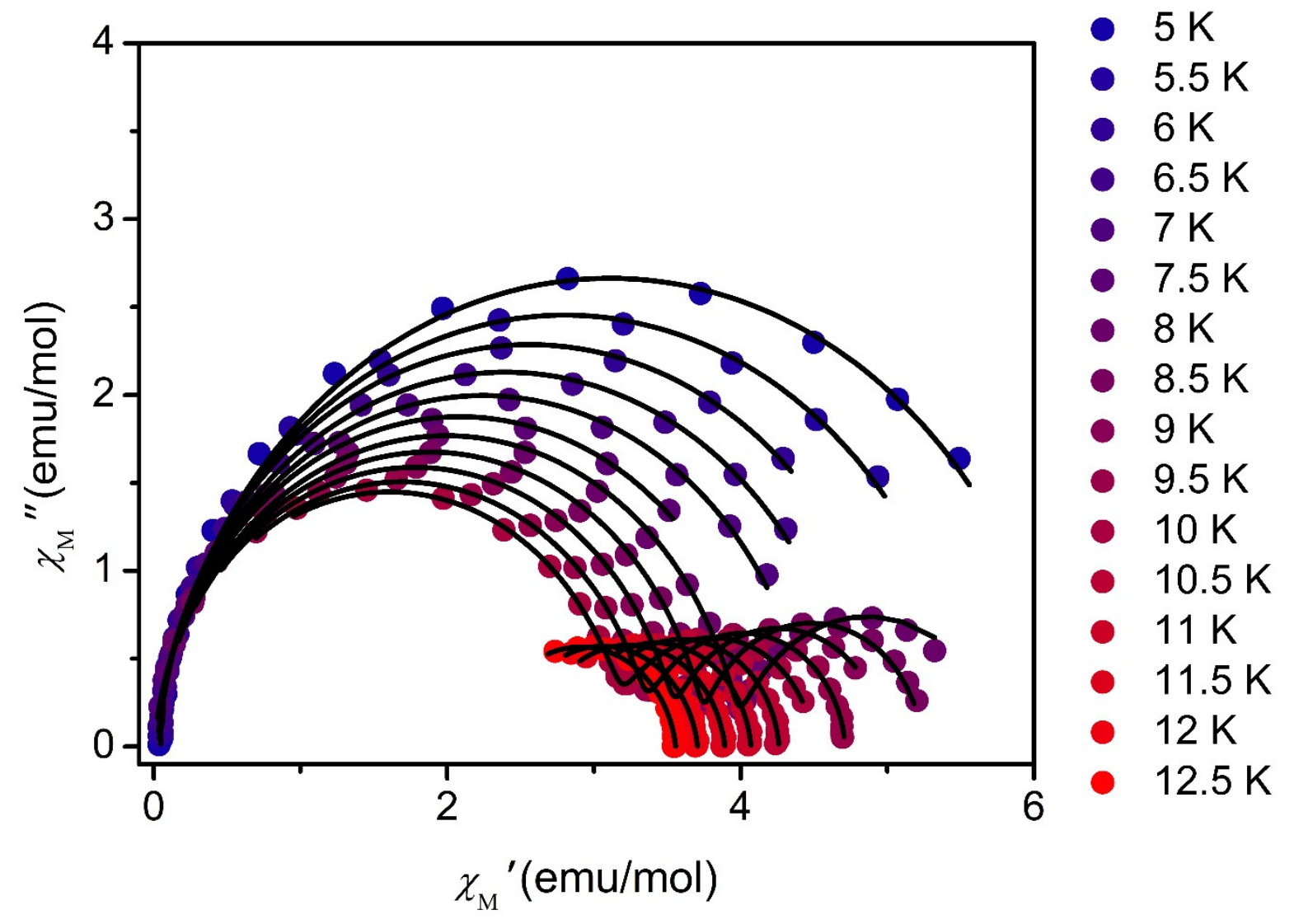

Figure S55. Cole-Cole plots for 2-Dy from 5.0-12.5 K (0.5 K steps). The black lines represent fits to the data using a generalized Debye model, which were used to extract $\tau$ values at each temperature. 


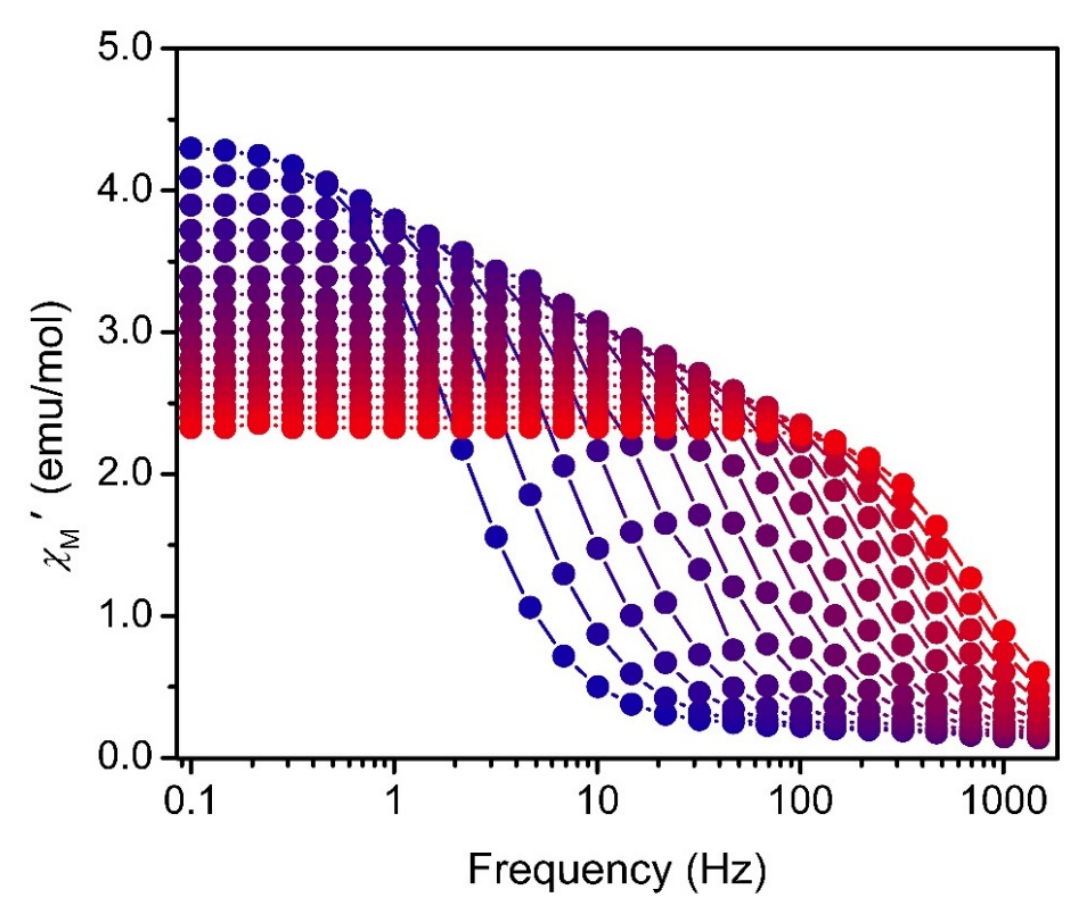

- $1.5 \mathrm{~K}$
- $10 \mathrm{~K}$
- $11 \mathrm{~K}$
- $11.5 \mathrm{~K}$
- $12 \mathrm{~K}$
- $12.5 \mathrm{~K}$
- $13 \mathrm{~K}$
- $13.5 \mathrm{~K}$
- $14 \mathrm{~K}$
- $14.5 \mathrm{~K}$
- $15 \mathrm{~K}$
- $15.5 \mathrm{~K}$
- $16 \mathrm{~K}$
- $16.5 \mathrm{~K}$
- $17 \mathrm{~K}$
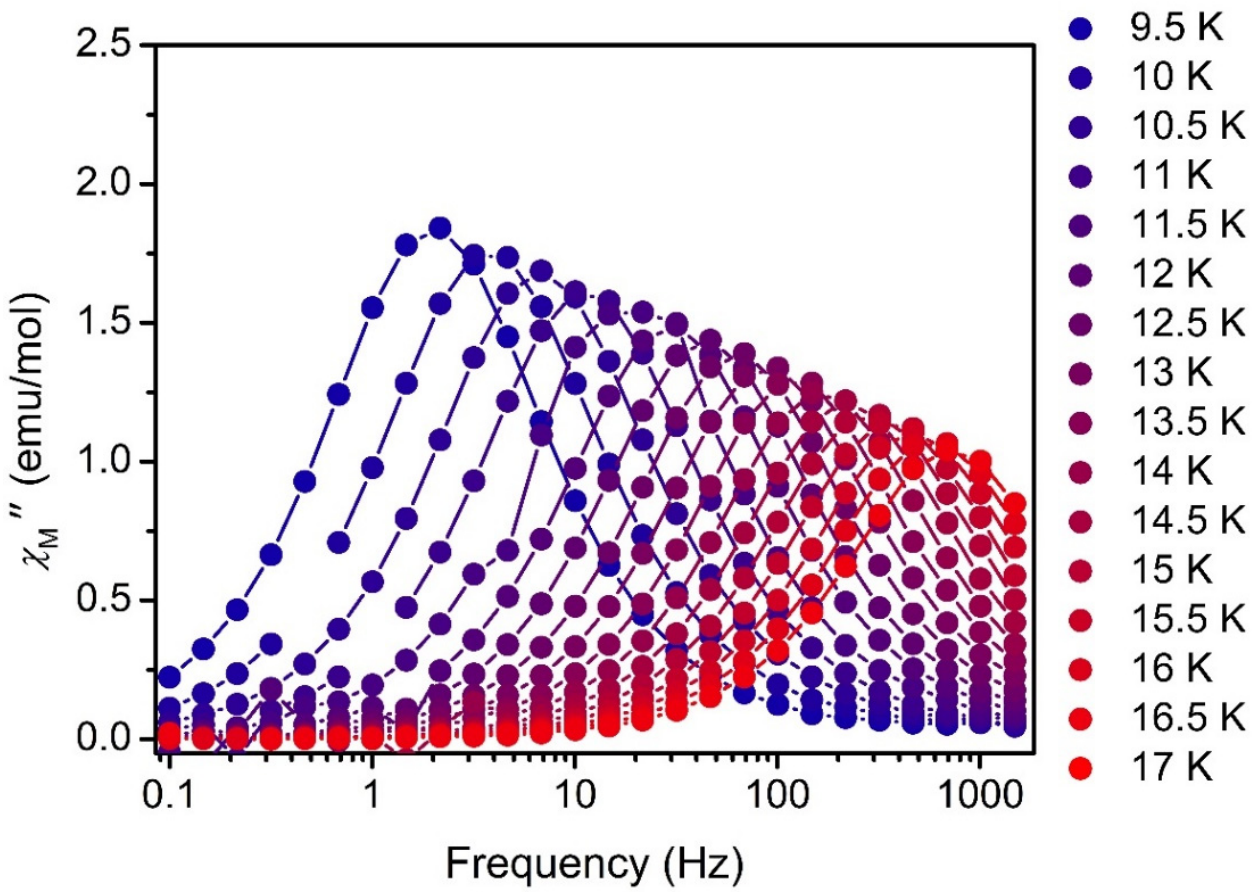

Figure S56. In-phase $\left(\chi_{\mathrm{m}^{\prime}}\right.$, top) and out-of-phase $\left(\chi_{\mathrm{M}}{ }^{\prime \prime}\right.$, bottom) components of the ac magnetic susceptibility for 3-Dy under zero applied de field at frequencies ranging from $0.1-1500 \mathrm{~Hz}$ and temperatures from $9.5-17.0 \mathrm{~K}(0.5 \mathrm{~K}$ steps $)$. The colored lines are guides for the eye. 


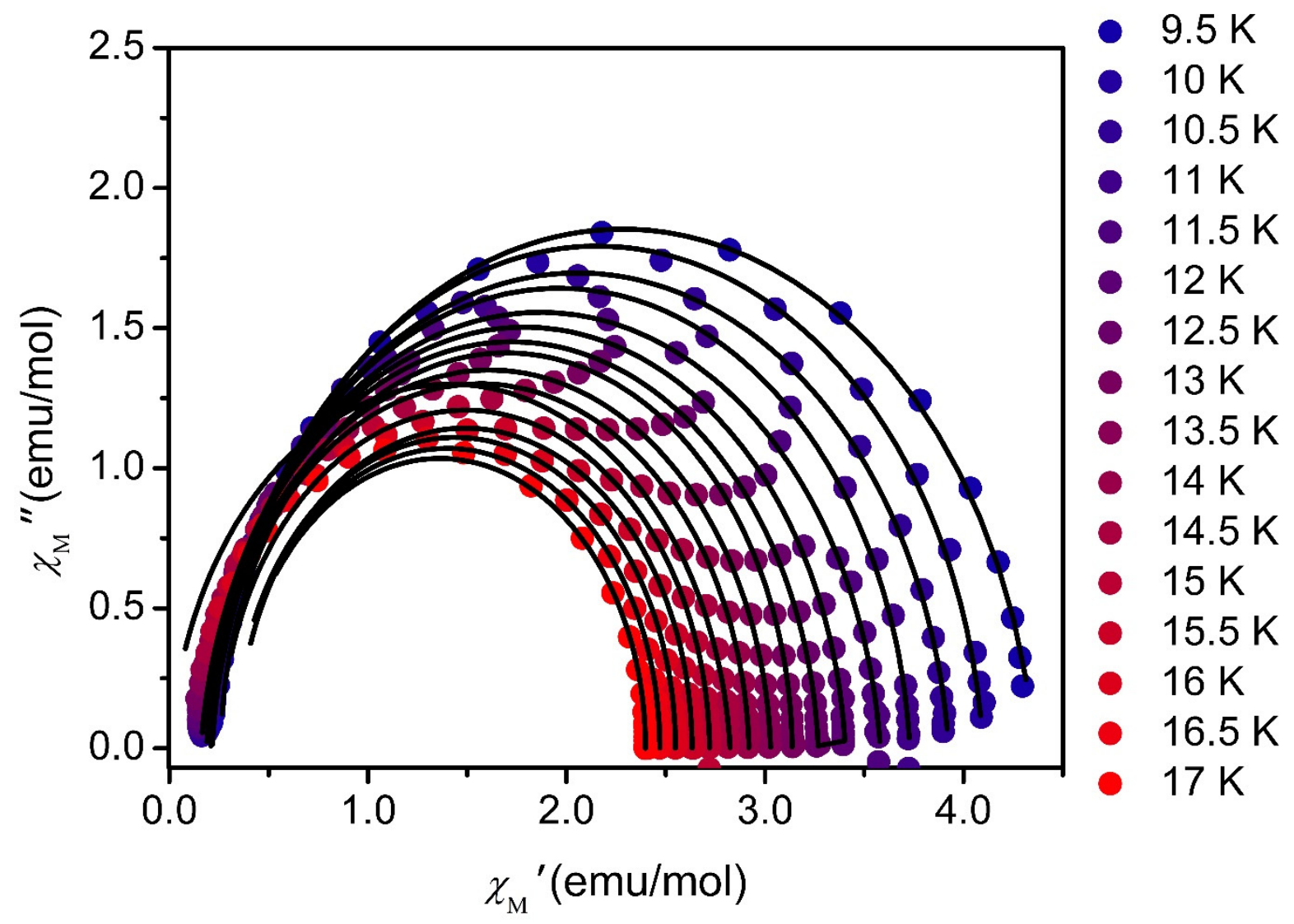

Figure S57. Cole-Cole plots for 3-Dy from 9.5-17.0 K (0.5 K steps). The black lines represent fits to the data using a generalized Debye model, which were used to extract $\tau$ values at each temperature. 


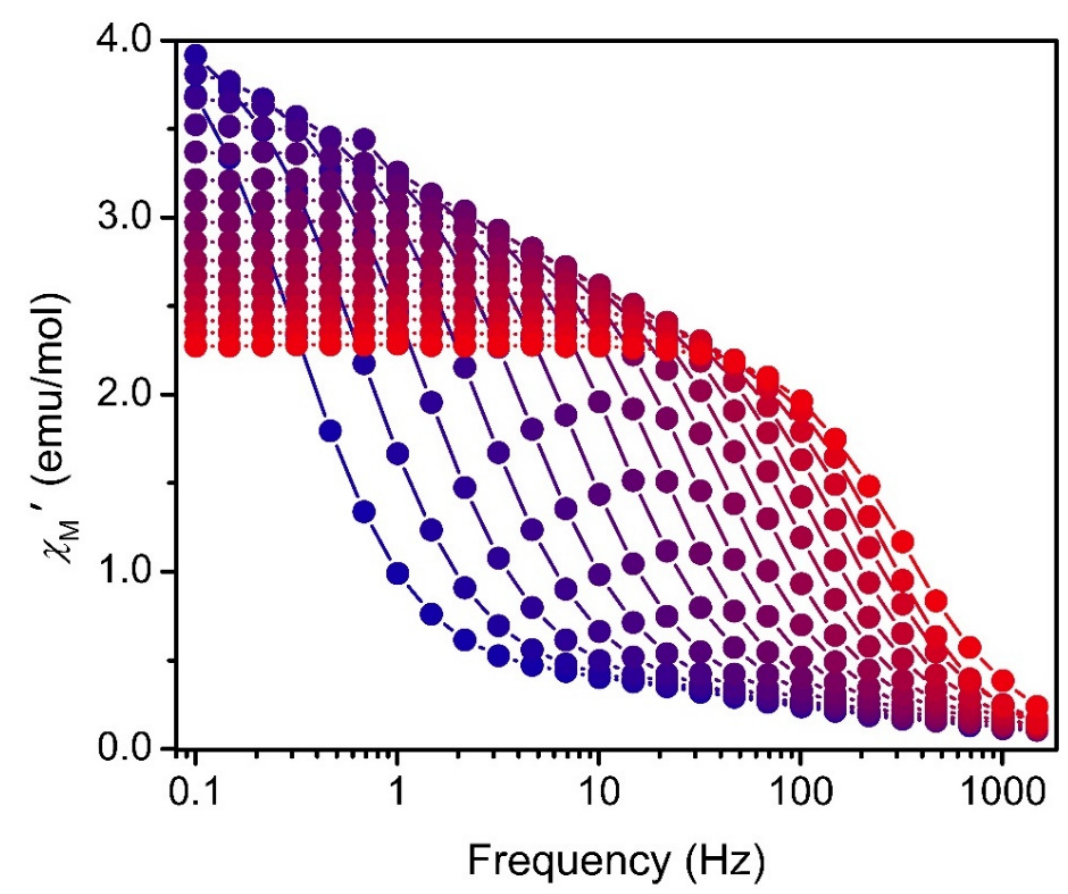

- $9 \mathrm{~K}$
- $9.5 \mathrm{~K}$
- $10 \mathrm{~K}$
- $10.5 \mathrm{~K}$
- $11 \mathrm{~K}$
- $11.5 \mathrm{~K}$
- $12 \mathrm{~K}$
- $12.5 \mathrm{~K}$
- $13 \mathrm{~K}$
- $13.5 \mathrm{~K}$
- $14 \mathrm{~K}$
- $15 \mathrm{~K}$
- $15.5 \mathrm{~K}$
- $16 \mathrm{~K}$
- $16.5 \mathrm{~K}$
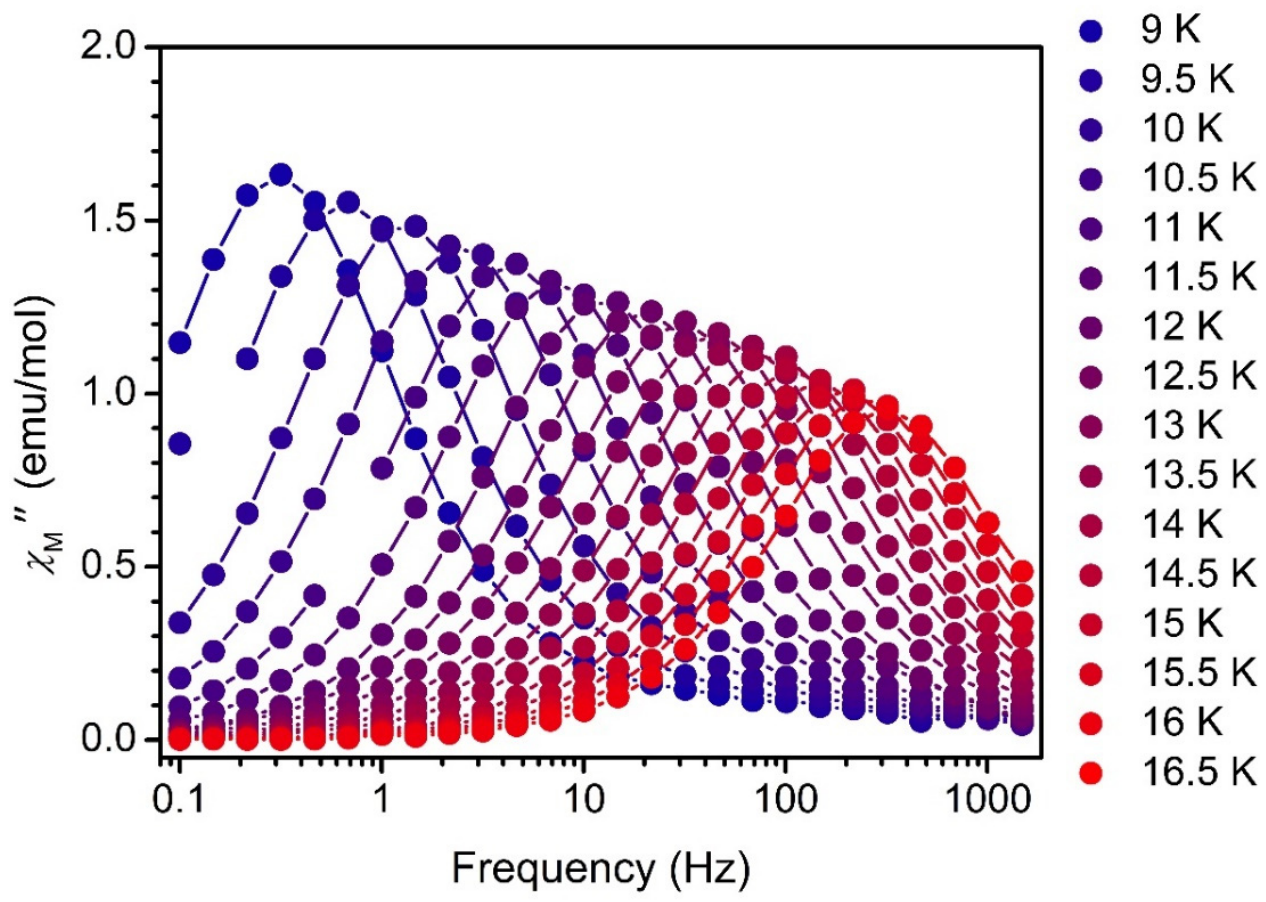

Figure S58. In-phase $\left(\chi \mathrm{m}^{\prime}\right.$, top) and out-of-phase $\left(\chi \mathrm{m}^{\prime \prime}\right.$, bottom) components of the ac magnetic susceptibility for 4-Dy under zero applied dc field at frequencies ranging from $0.1-1500 \mathrm{~Hz}$ and temperatures from $9.0-16.5 \mathrm{~K}(0.5 \mathrm{~K}$ steps $)$. The colored lines are guides for the eye. 


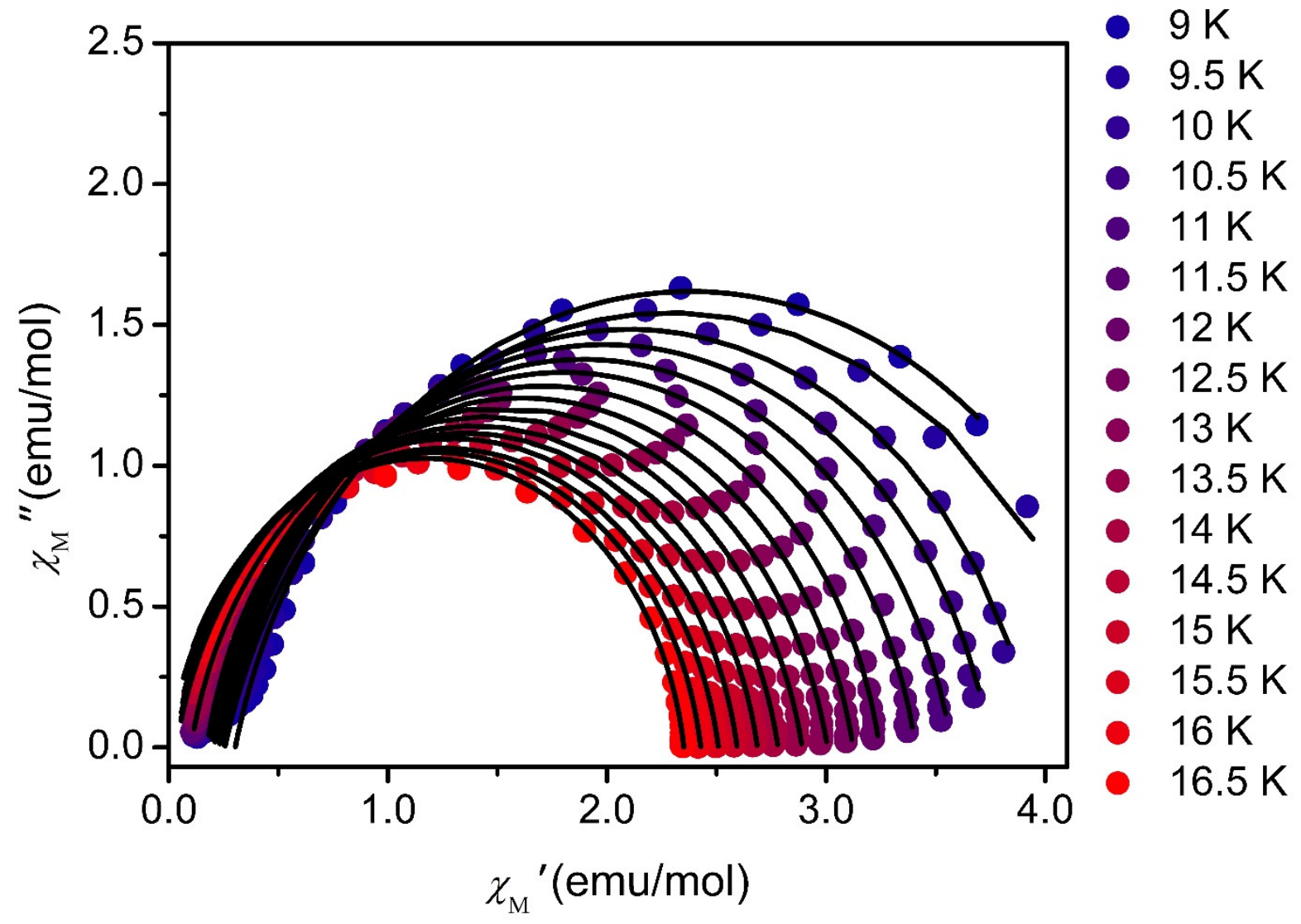

Figure S59. Cole-Cole plots for 4-Dy from 9.0-16.5 K (0.5 K steps). The black lines represent fits to the data using a generalized Debye model, which were used to extract $\tau$ values at each temperature. 

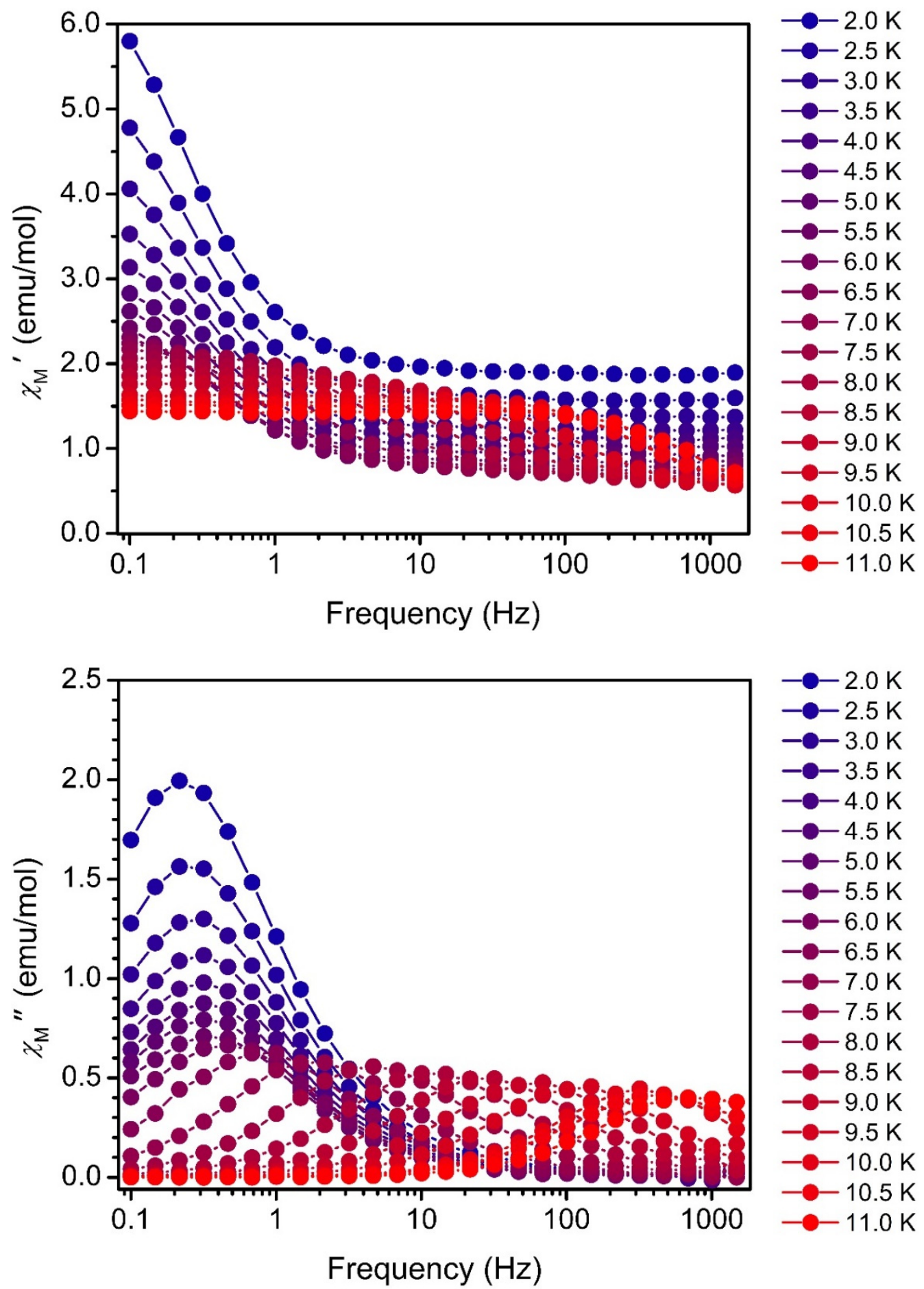

Figure S60. In-phase $\left(\chi_{\mathrm{m}^{\prime}}\right.$, top) and out-of-phase $\left(\chi_{\mathrm{m}}\right.$ ", bottom) components of the ac magnetic susceptibility for 4-Tb under zero applied de field at frequencies ranging from $0.1-1500 \mathrm{~Hz}$ and temperatures from $2.0-11.0 \mathrm{~K}(0.5 \mathrm{~K}$ steps $)$. The colored lines are guides for the eye. 


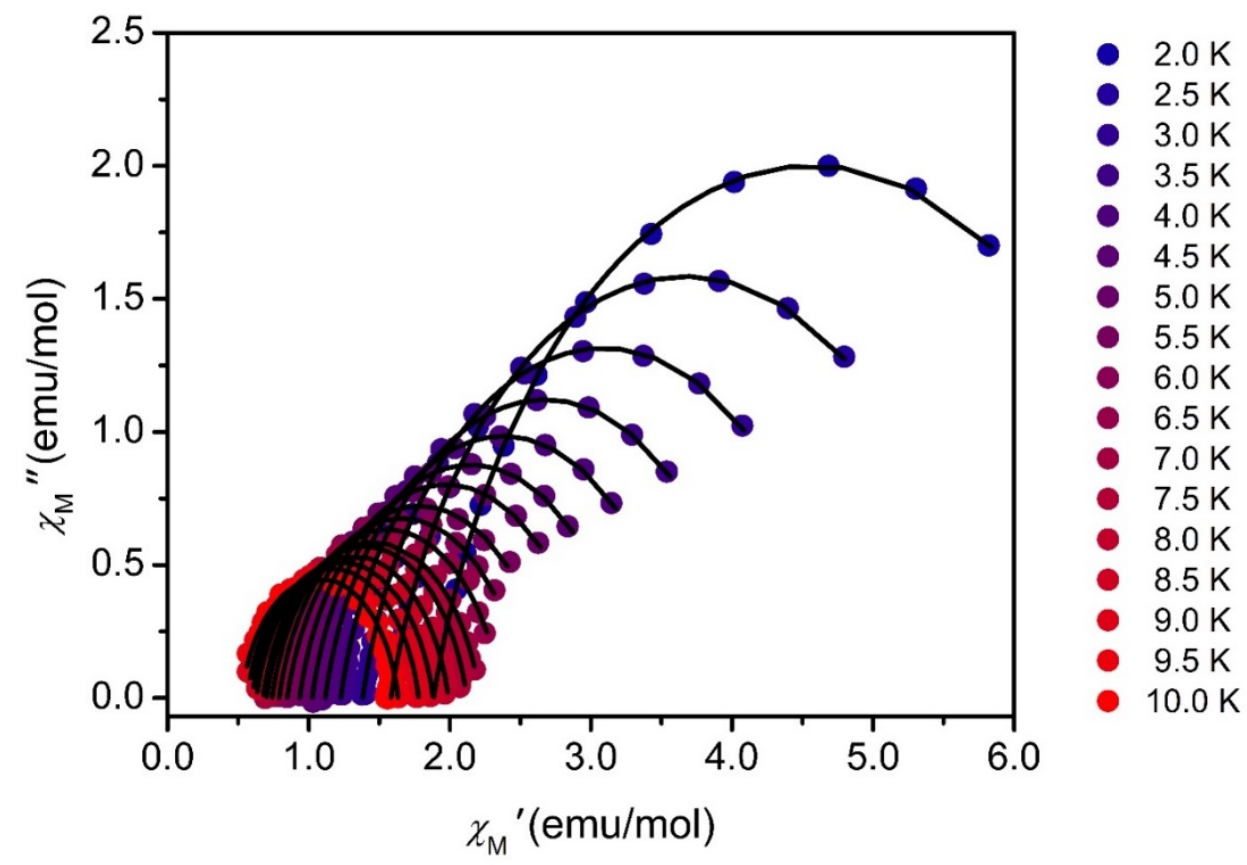

Figure S61. Cole-Cole plots for 4-Tb from 2.0-10.0 K (0.5 K steps). The black lines represent fits to the data using a generalized Debye model, which were used to extract $\tau$ values at each temperature.

\section{Section S5.3 Dc Magnetic Relaxation Data}

Dc magnetic relaxation data were collected for 3-Dy and 4-Dy after magnetizing a sample at $5 \mathrm{~T}$ for $5 \mathrm{~min}$ and then zeroing the field (no overshoot, hi-res disabled). The magnetization was then measured at periodic time intervals. Dc magnetic relaxation data were fit to a stretched exponential function:

$$
M(t)=M_{1}+\left(M_{0}-M_{1}\right)^{(t / \tau)^{n}}
$$

where $M(t)$ is the magnetization at time $t, M_{0}$ is the initial magnetization measured after the field has been removed, $M_{1}$ is the final value of the magnetization at $t=\infty, \tau$ is the magnetic relaxation time, and $n$ is a free variable. For data collected under a field of $500 \mathrm{Oe}, M_{1}$ was independently determined by dc susceptibility measurements. For data collected after the field was set to zero, $M_{1}$ should equal zero, however, non-zero values were obtained for all dc magnetic relaxation measurements. This likely arises due to a small remnant field. We therefore set $M_{1}$ equal to the value of the magnetization measured at the last time point of each experiment. At high temperatures, it was possible to measure the full decay of the magnetization within $5000 \mathrm{~s}$. At lower temperatures, however, magnetic relaxation was significantly slower and thus this length of time was not sufficient to observe the full decay. For these measurements, setting $M_{1}$ equal to the last value of the magnetization measured for these experiments represents an overestimate. Better fits to the data for these experiments were obtained by treating $M_{1}$ as a free variable ( $M_{1}$ values marked with an asterisk in the tables below).

In order to test the validity of this strategy, fits to higher temperature experiments with $M_{1}$ as a free variable were obtained and compared to fits where $M_{1}$ was fixed equal to the last value of the magnetization measured. This resulted in values of $\tau$ within $5 \%$ error for the two fits. Furthermore, the values obtained for $\tau$ with $M_{1}$ as a free variable were consistently lower than those obtained 
with $M_{1}$ equal to the last value of the magnetization, demonstrating that this alternative fitting strategy did not overestimate the value of $\tau$ at low temperatures.

The $n$ values extracted from fits to dc relaxation data were used to determine uncertainty ranges in $\tau$ according to the following equation:

$$
\tau_{ \pm}(1 \sigma)=\tau_{\mu} e^{ \pm \frac{1.64 \tan \left[\frac{\pi}{2}(1-n)\right]}{(1-n)^{0.141}}}
$$

where $\tau_{ \pm}(1 \sigma)$ is the uncertainty range for one standard deviation from the mean and $\tau_{\mu}$ is the mean relaxation time. ${ }^{2}$ As with the $\tau_{ \pm}(1 \sigma)$ values obtained for ac susceptibility measurements, these uncertainty ranges are not a reflection of experimental error, but instead represent a distribution of relaxation times that is intrinsic to a compound.

Data for 1-Dy and 2-Dy could not be fit to a single stretched exponential function. It is likely that two competing relaxation pathways exist for these complexes in the low-temperature regime, as was observed in ac susceptibility measurements. Attempts to fit the data using a stretched exponential function that accounts for two relaxation processes led to over-parameterization.

Table S12. Parameters used to fit de magnetic relaxation data for a solid sample of 3-Dy collected under zero applied magnetic field and magnetic relaxation times extracted from these fits.

\begin{tabular}{ccccc}
\hline $\boldsymbol{T}(\mathbf{K})$ & $\boldsymbol{M}_{\mathbf{0}}\left(\boldsymbol{\mu}_{\mathbf{b}}\right)$ & $\boldsymbol{M}_{\mathbf{1}}\left(\boldsymbol{\mu}_{\mathbf{b}}\right)$ & $\boldsymbol{n}$ & $\boldsymbol{\tau}(\mathbf{s})^{a}$ \\
\hline 2 & 4.84269 & $0.35440^{*}$ & 0.558 & 662.481 \\
2.5 & 4.53015 & $0.28013^{*}$ & 0.579 & 596.692 \\
3 & 4.35195 & $0.20986^{*}$ & 0.585 & 554.546 \\
3.5 & 4.21606 & $0.15901^{*}$ & 0.592 & 521.259 \\
4 & 4.10886 & $0.11277^{*}$ & 0.603 & 485.203 \\
4.5 & 3.96565 & 0.04817 & 0.623 & 448.889 \\
5 & 3.65953 & 0.01694 & 0.720 & 309.010 \\
5.5 & 2.55888 & 0.00656 & 0.804 & 142.559 \\
6 & 0.60338 & 0.00429 & 0.835 & 68.025 \\
\hline
\end{tabular}

${ }^{a}$ Values for $\tau$ are likely only accurate to three significant figures. The full value extracted from the fit is reported for reproducibility. 


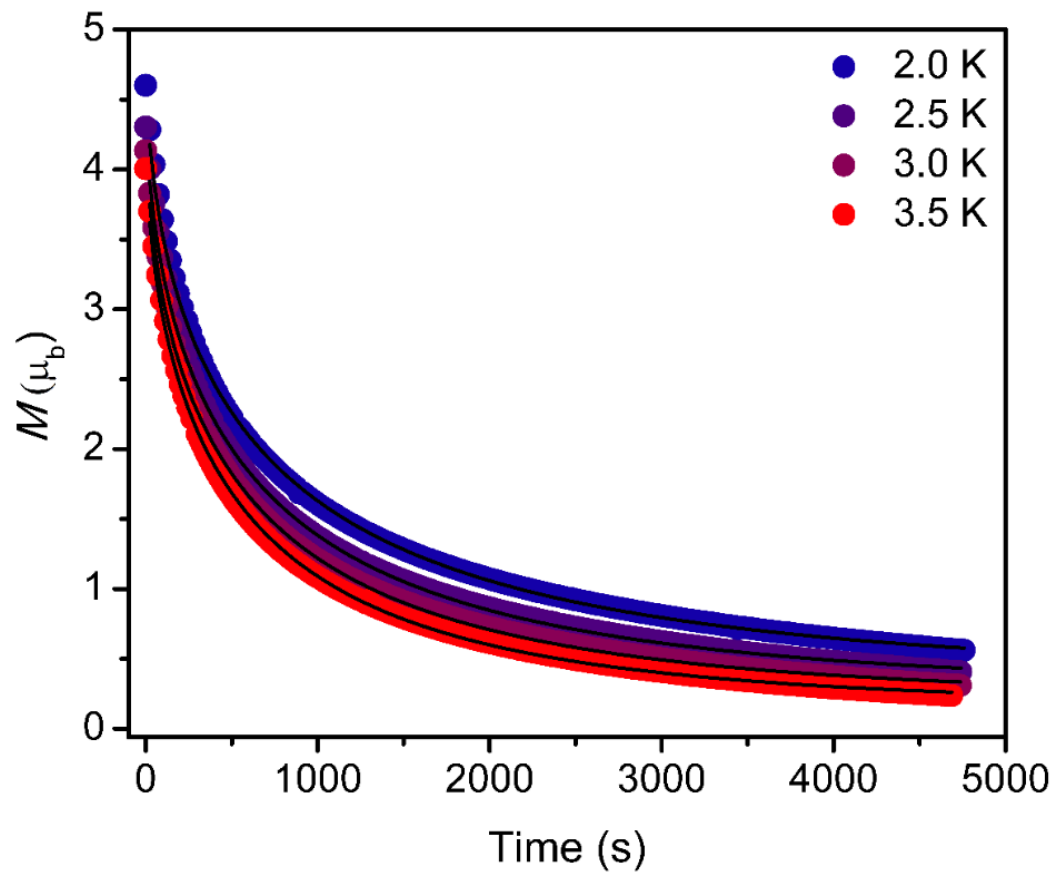

Figure S62. Dc magnetic relaxation data for a solid sample of 3-Dy collected at 2.0, 2.5, 3.0, and $3.5 \mathrm{~K}$ under zero field after magnetization at $5 \mathrm{~T}$. Black lines represent the fits to the data used to extract $\tau$.

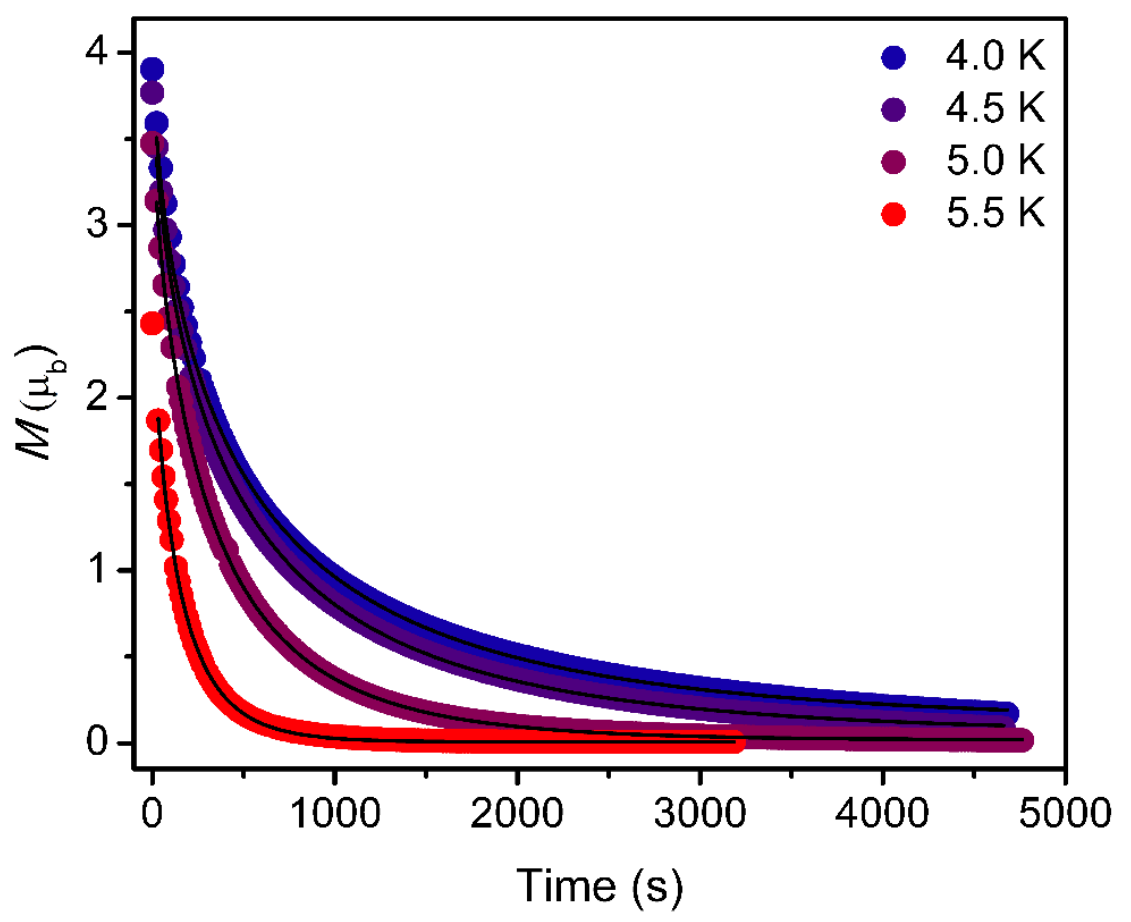

Figure S63. Dc magnetic relaxation data for a solid sample of 3-Dy collected at 4.0, 4.5, 5.0, and $5.5 \mathrm{~K}$ at zero field following magnetization at $5 \mathrm{~T}$. Black lines represent the fits to the data using stretched exponential functions, which were used to extract $\tau$. 


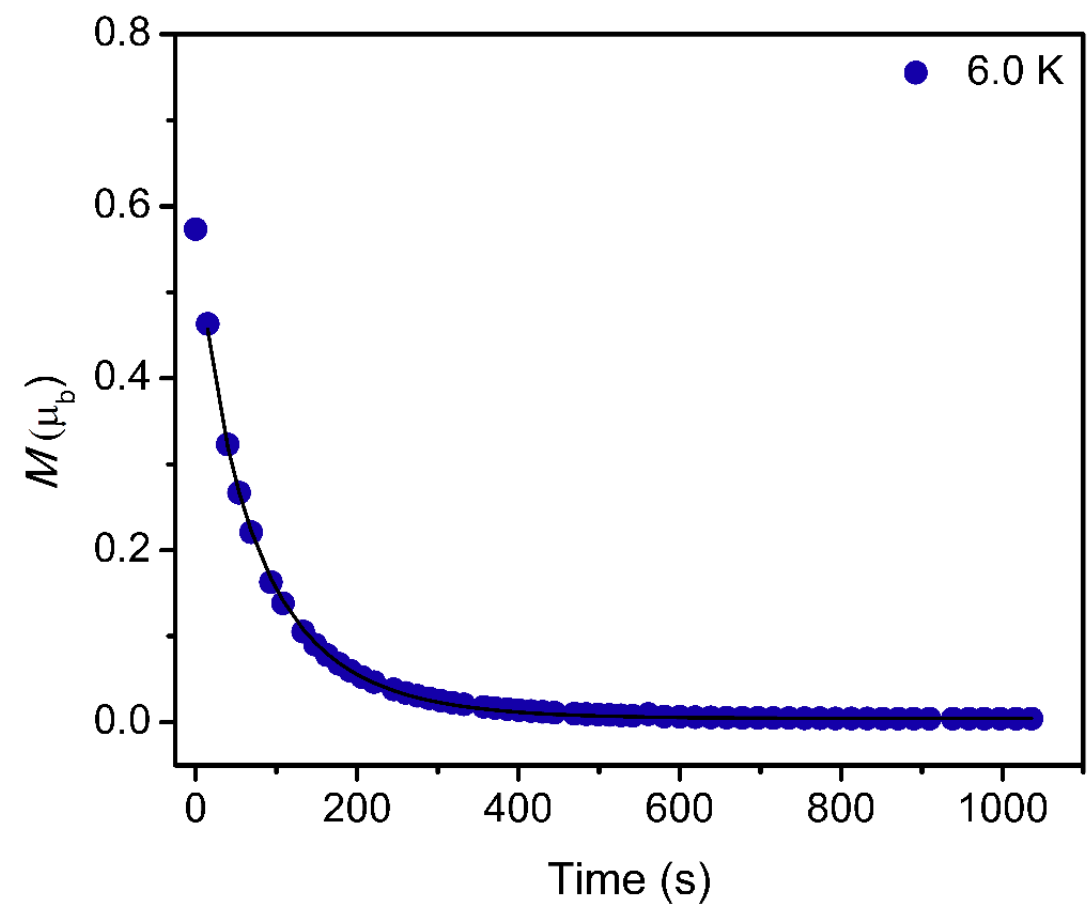

Figure S64. De magnetic relaxation data for a solid sample of 3-Dy collected at $6 \mathrm{~K}$ under zero field after magnetization at $5 \mathrm{~T}$. Black line represents the fit to the data using stretched exponential functions, which was used to extract $\tau$.

Table S13. Parameters used to fit de magnetic relaxation data for a $7.8 \mathrm{mM}$ solution of 3-Dy collected under zero applied field and magnetic relaxation times extracted from these fits.

\begin{tabular}{ccccc}
\hline $\boldsymbol{T}(\mathbf{K})$ & $\boldsymbol{M}_{\mathbf{0}}\left(\boldsymbol{\mu}_{\mathbf{b}}\right)$ & $\boldsymbol{M}_{\mathbf{1}}\left(\boldsymbol{\mu}_{\mathbf{b}}\right)$ & $\boldsymbol{n}$ & $\boldsymbol{\tau}(\mathbf{s})^{a}$ \\
\hline 2 & 1.10268 & 0.04406 & 0.782 & 96.274 \\
2.5 & 1.01419 & 0.03249 & 0.784 & 92.038 \\
3 & 0.86536 & 0.01996 & 0.782 & 92.343 \\
3.5 & 0.82655 & 0.01970 & 0.796 & 91.620 \\
4.5 & 0.72785 & 0.01698 & 0.798 & 86.801 \\
5 & 0.59102 & 0.00924 & 0.798 & 79.567 \\
5.5 & 0.29494 & 0.00606 & 0.810 & 64.266
\end{tabular}

${ }^{a}$ Values for $\tau$ are likely only accurate to three significant figures. The full value extracted from the fit is reported for reproducibility. 


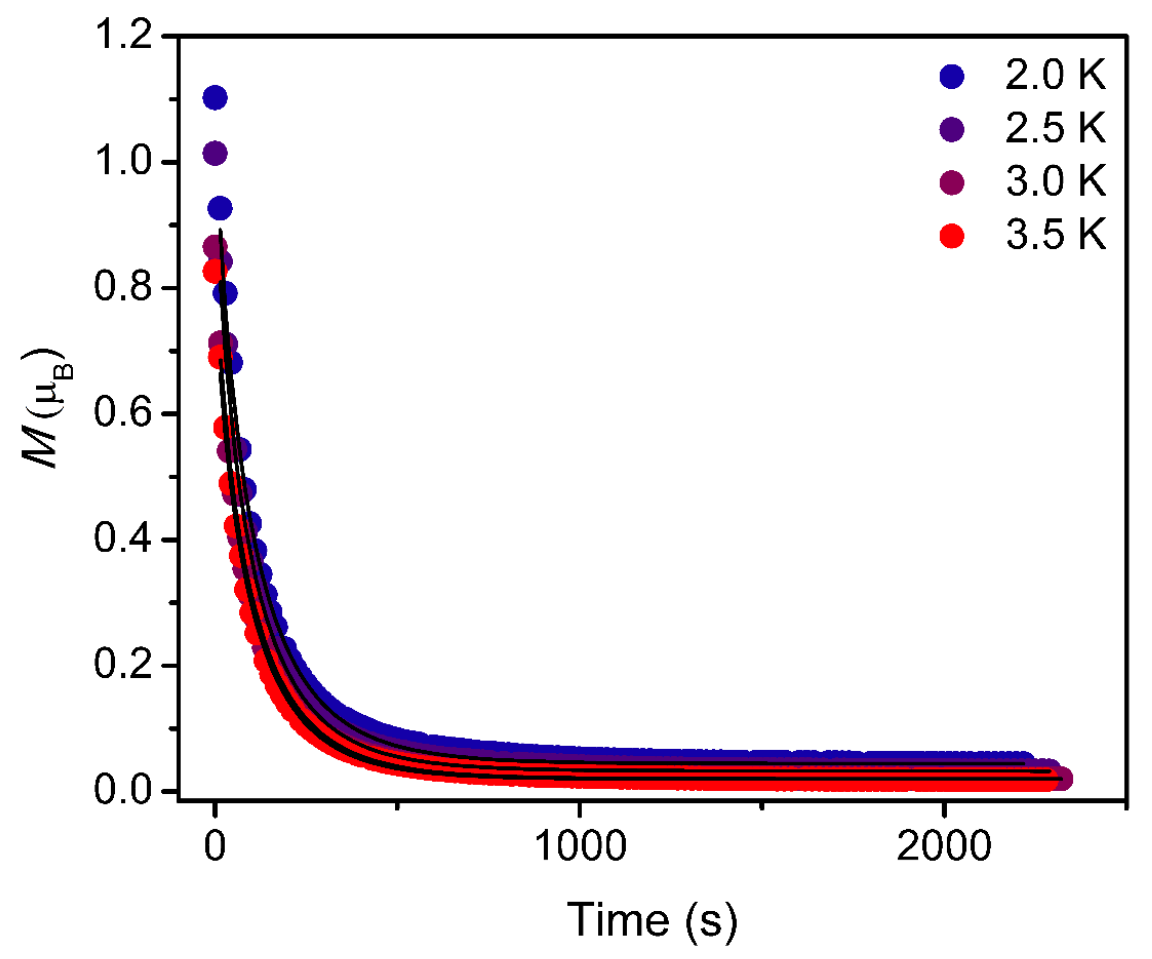

Figure S65. Dc magnetic relaxation data for a $7.8 \mathrm{mM}$ of 3-Dy collected at 2.0, 2.5, 3.0, and 3.5 $\mathrm{K}$ under zero field after magnetization at $5 \mathrm{~T}$. Black lines represent the fits to the data using stretched exponential functions, which were used to extract $\tau$.

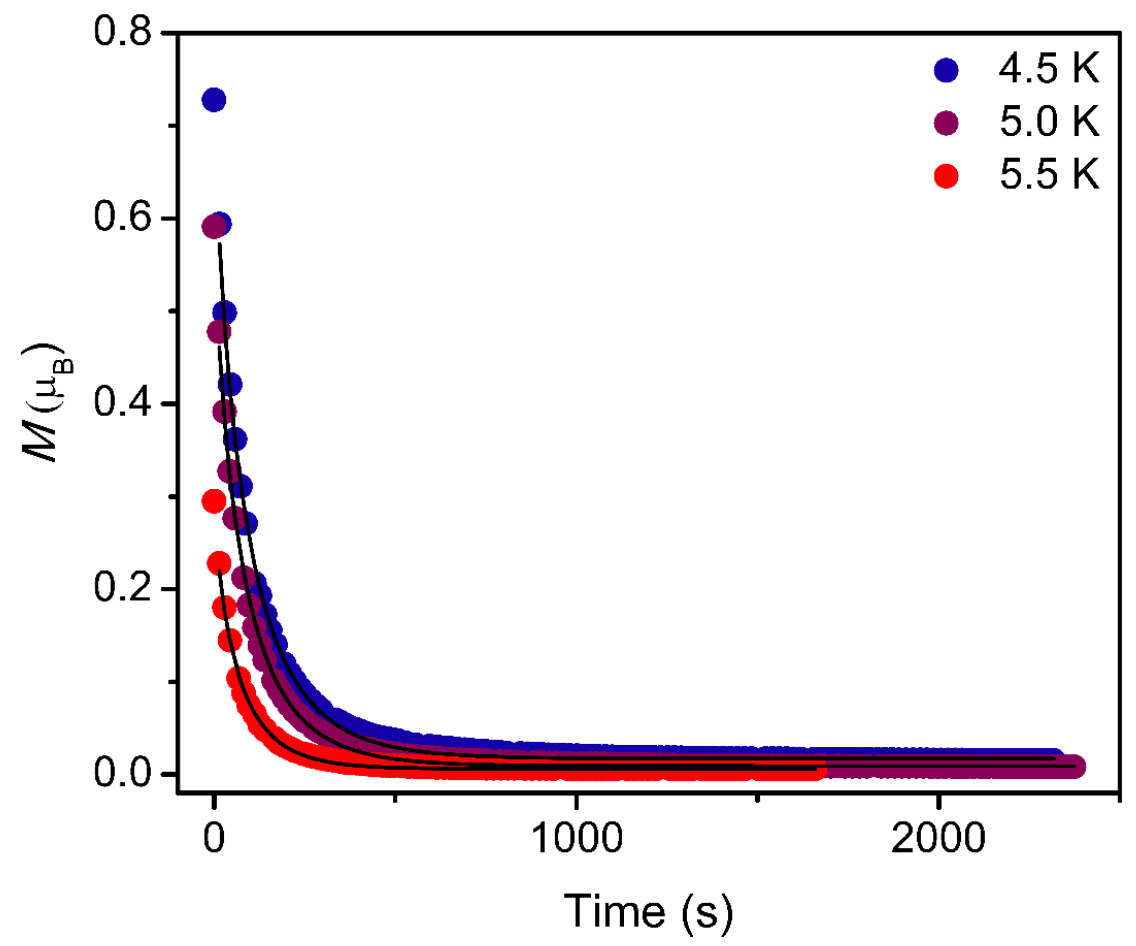

Figure S66. De magnetic relaxation data for a $7.8 \mathrm{mM}$ of 3-Dy collected at 4.5, 5.0, and $5.5 \mathrm{~K}$ under zero field after magnetization at $5 \mathrm{~T}$. Black lines represent the fits to the data using stretched exponential functions, which were used to extract $\tau$. 
Table S14. Parameters used to fit de magnetic relaxation data for a $7.8 \mathrm{mM}$ solution of 3-Dy collected under 500 Oe and magnetic relaxation times extracted from these fits.

\begin{tabular}{ccccc}
\hline $\boldsymbol{T}(\mathbf{K})$ & $\boldsymbol{M}_{\mathbf{0}}\left(\boldsymbol{\mu}_{\mathbf{b}}\right)$ & $\boldsymbol{M}_{\mathbf{1}}\left(\boldsymbol{\mu}_{\mathbf{b}}\right)$ & $\boldsymbol{n}$ & $\boldsymbol{\tau}(\mathbf{s})^{a}$ \\
\hline 2 & 5.67369 & 1.01498 & 0.440 & 38386.401 \\
2.5 & 5.54410 & 0.88025 & 0.441 & 30165.846 \\
3 & 5.40343 & 0.77994 & 0.452 & 25713.768 \\
3.5 & 5.29255 & 0.72273 & 0.471 & 18975.266 \\
4 & 5.16208 & 0.68211 & 0.511 & 12573.178 \\
4.5 & 4.91838 & 0.63007 & 0.608 & 4508.859 \\
5 & 4.10393 & 0.59884 & 0.657 & 1252.540 \\
5.5 & 2.33356 & 0.61981 & 0.745 & 335.735 \\
6 & 0.92102 & 0.55783 & 0.827 & 130.599 \\
\hline
\end{tabular}

${ }^{a}$ Values for $\tau$ are likely only accurate to three significant figures. The full value extracted from the fit is reported for reproducibility.

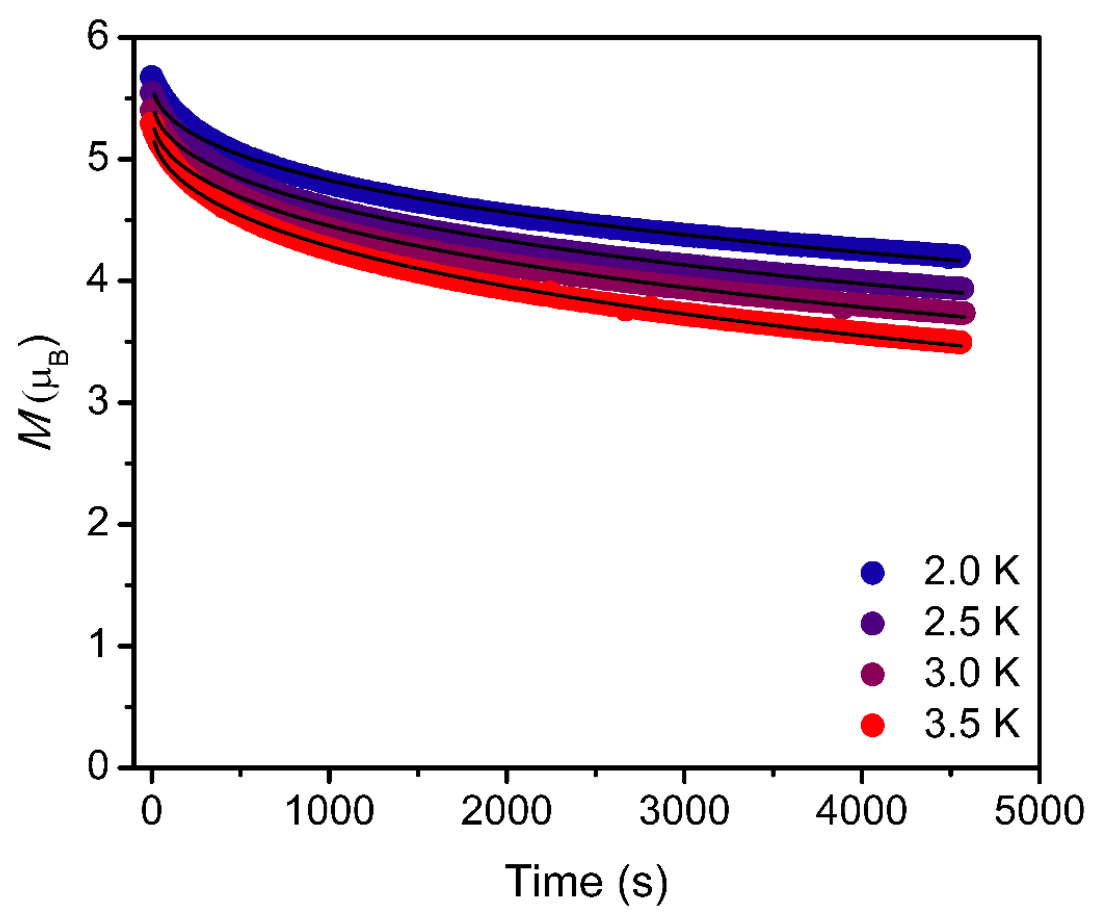

Figure S67. De magnetic relaxation data for a $7.8 \mathrm{mM}$ of 3-Dy collected at 2.0, 2.5, 3.0, and 3.5 $\mathrm{K}$ under a 500 Oe field. Black lines represent the fits to the data using stretched exponential functions, which were used to extract $\tau$. 


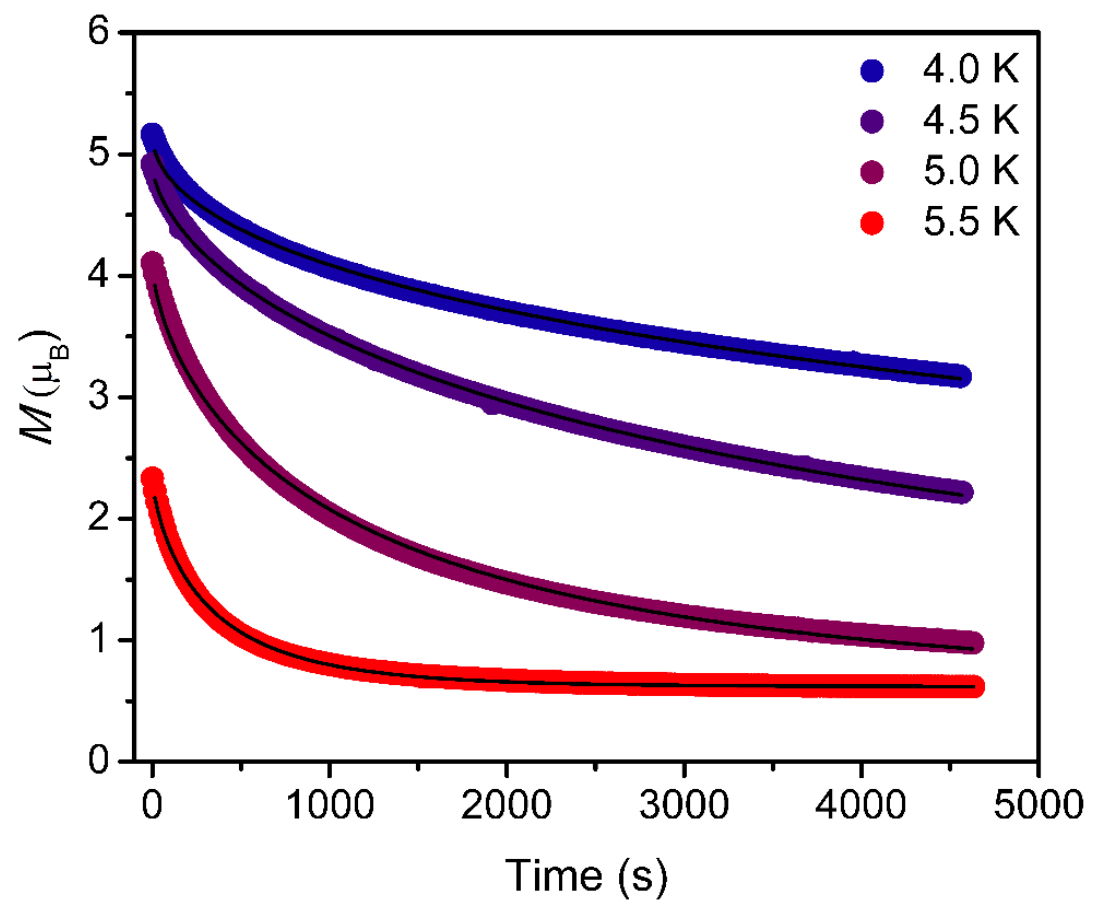

Figure S68. De magnetic relaxation data for a 7.8 $\mathrm{mM}$ of 3-Dy collected at 4.0, 4.5, 5.0, and 5.5 $\mathrm{K}$ under a 500 Oe field. Black lines represent the fits to the data using stretched exponential functions, which were used to extract $\tau$.

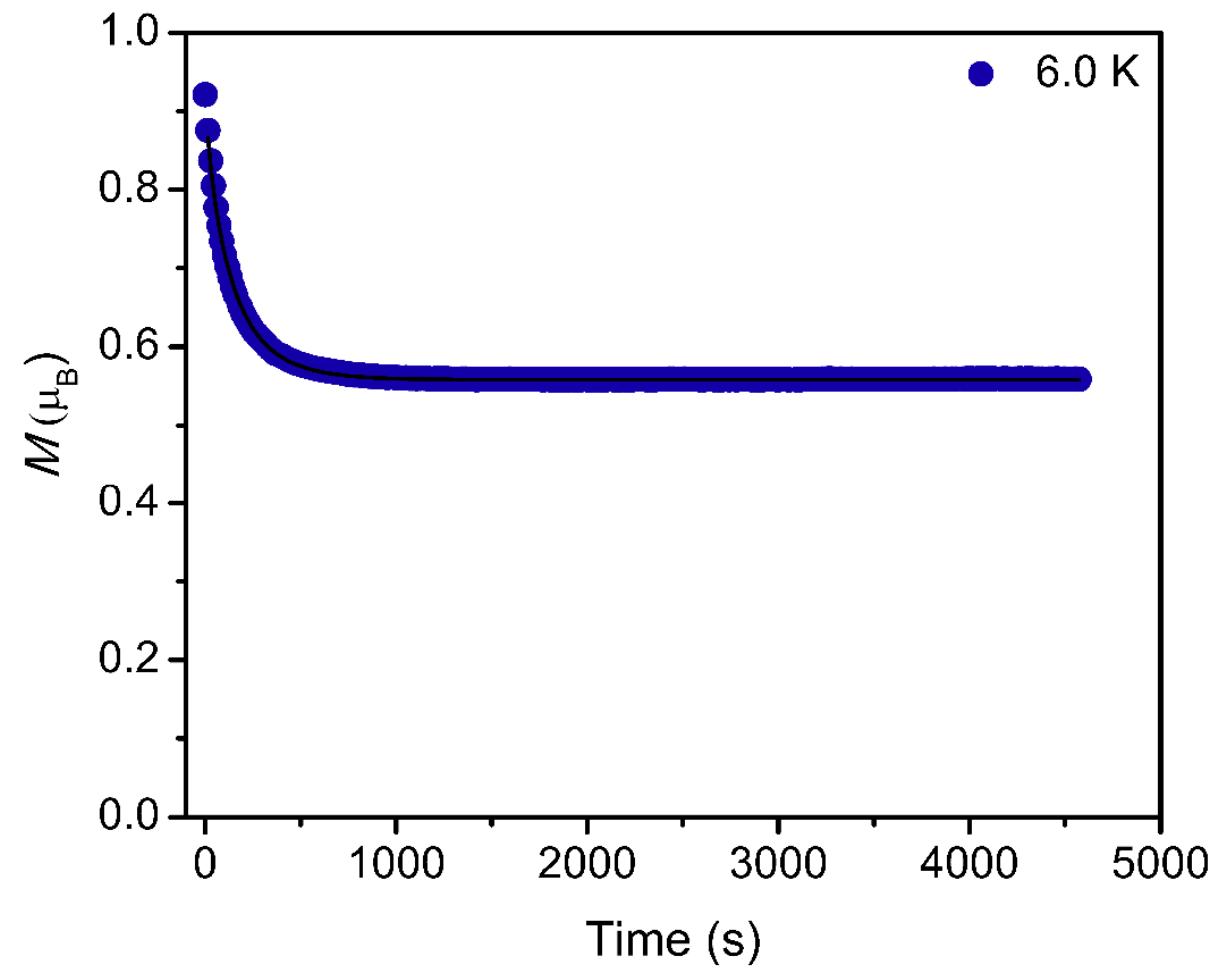

Figure S69. Dc magnetic relaxation data for a $7.8 \mathrm{mM}$ of 3-Dy collected at $6.0 \mathrm{~K}$ under a 500 Oe field. The black line represent the fit to the data using a stretched exponential function, which was used to extract $\tau$. 
Table S15. Parameters used to fit dc magnetic relaxation data for a solid sample of 4-Dy collected under zero applied magnetic field and magnetic relaxation times extracted from these fits.

\begin{tabular}{ccccc}
\hline $\boldsymbol{T}(\mathbf{K})$ & $\boldsymbol{M}_{\mathbf{0}}\left(\boldsymbol{\mu}_{\mathbf{b}}\right)$ & $\boldsymbol{M}_{\mathbf{1}}\left(\boldsymbol{\mu}_{\mathbf{b}}\right)$ & $\boldsymbol{n}$ & $\boldsymbol{\tau}(\mathbf{s})^{a}$ \\
\hline 2 & 3.05575 & 0.04728 & 0.720 & 353.920 \\
2.5 & 2.87448 & 0.02656 & 0.746 & 304.031 \\
3 & 2.97010 & 0.02242 & 0.751 & 255.975 \\
3.5 & 2.47644 & 0.01347 & 0.793 & 240.800 \\
4 & 2.31350 & 0.01144 & 0.816 & 218.358 \\
4.5 & 2.15091 & 0.00969 & 0.839 & 196.540 \\
5 & 1.96927 & 0.00883 & 0.873 & 171.347 \\
5.5 & 1.62353 & 0.00394 & 0.936 & 129.078 \\
6 & 0.92276 & 0.00363 & 0.960 & 79.613 \\
6.5 & 0.27924 & 0.00199 & 0.933 & 56.767 \\
\hline
\end{tabular}

${ }^{a}$ Values for $\tau$ are likely only accurate to three significant figures. The full value extracted from the fit is reported for reproducibility.

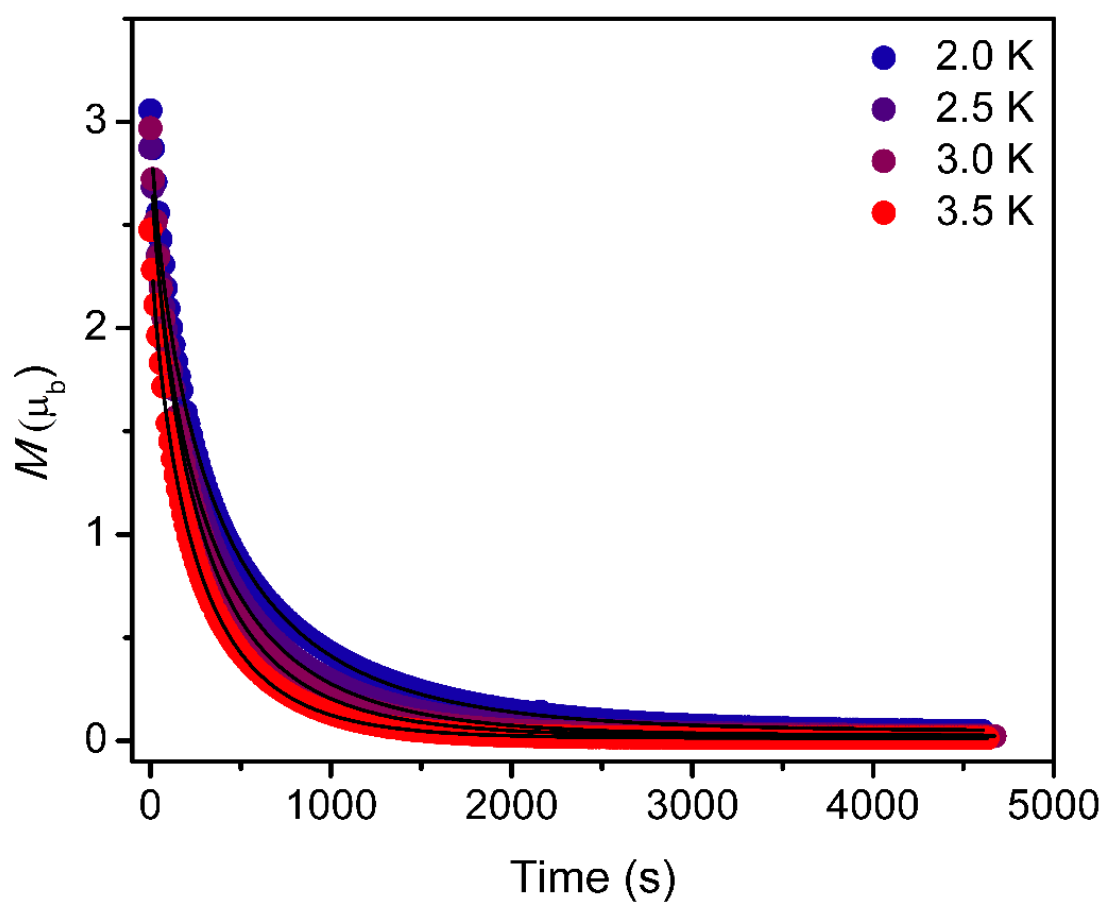

Figure S70. Dc magnetic relaxation data for a solid sample of 4-Dy collected at 2, 2.5, 3 and 3.5 $\mathrm{K}$ under zero field after magnetization at $5 \mathrm{~T}$. Black line represents the fit to the data using stretched exponential functions, which was used to extract $\tau$. 


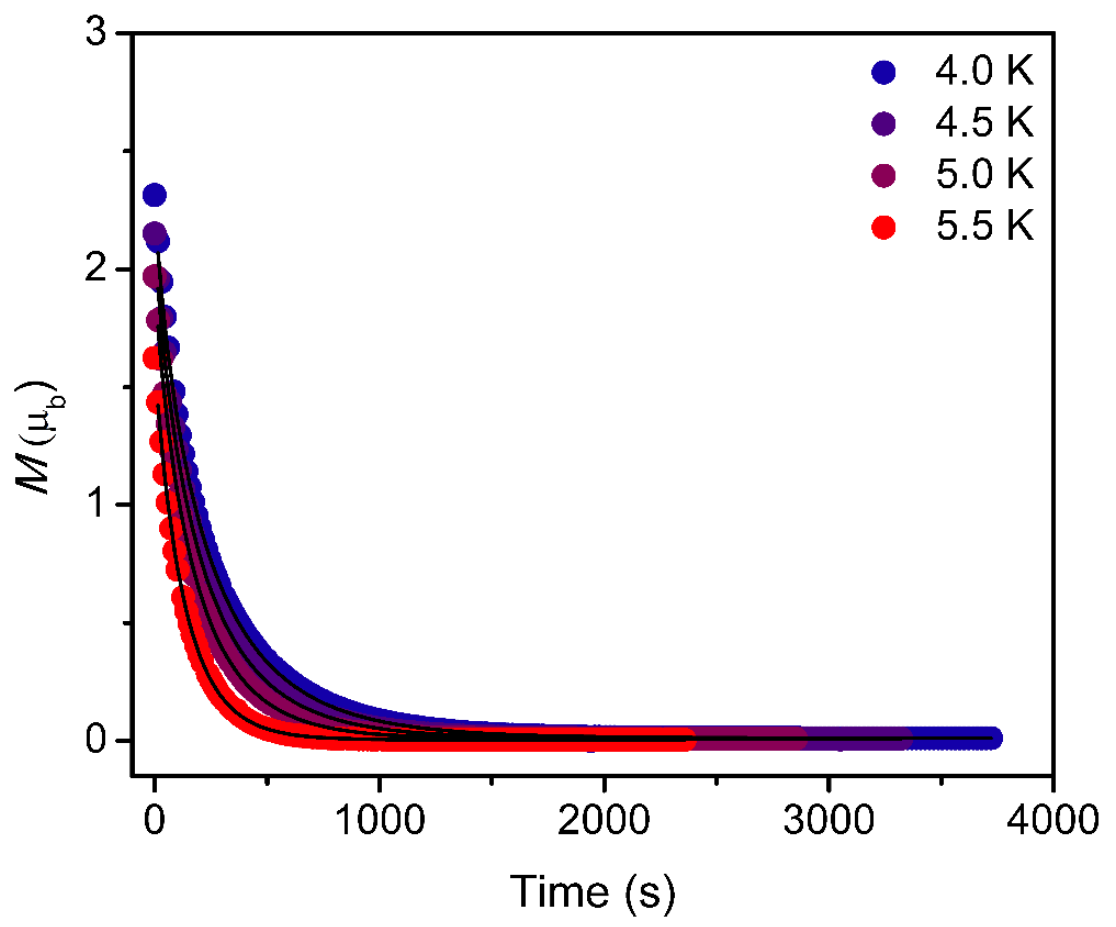

Figure S71. De magnetic relaxation data for a solid sample of 4-Dy collected at 4, 4.5, 5 and 5.5 $\mathrm{K}$ under zero field after magnetization at $5 \mathrm{~T}$. Black line represents the fit to the data using stretched exponential functions, which was used to extract $\tau$.

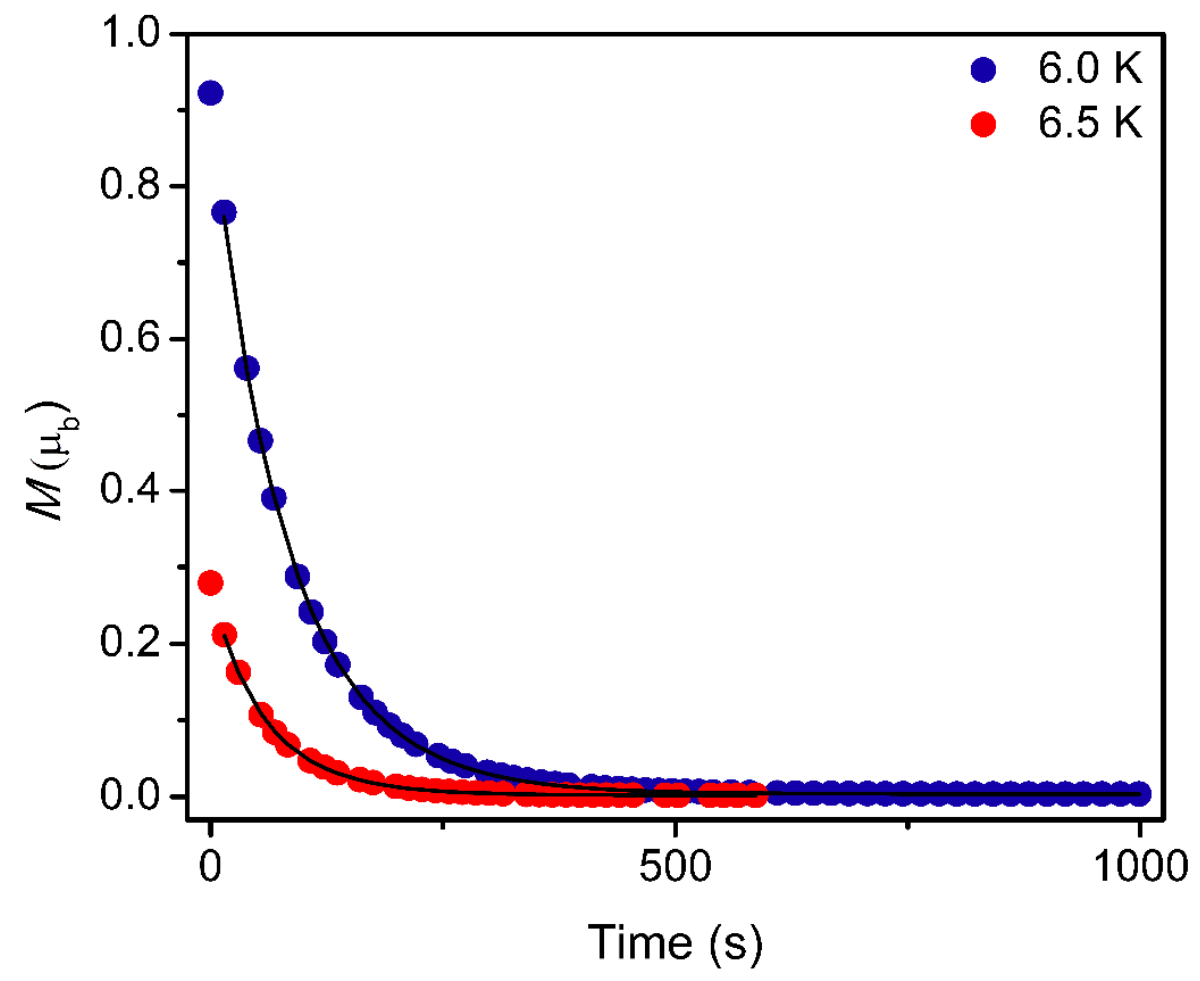

Figure S72. De magnetic relaxation data for a solid sample of 4-Dy collected at 6 and $6.5 \mathrm{~K}$ under zero field after magnetization a $5 \mathrm{~T}$. Black line represents the fit to the data using stretched exponential functions, which was used to extract $\tau$. 
Table S16. Parameters used to fit dc magnetic relaxation data for an $8.0 \mathrm{mM}$ solution of 4-Dy collected under zero applied field and magnetic relaxation times extracted from these fits.W

\begin{tabular}{ccccc}
\hline $\boldsymbol{T}(\mathbf{K})$ & $\boldsymbol{M}_{\mathbf{0}}\left(\boldsymbol{\mu}_{\mathbf{b}}\right)$ & $\boldsymbol{M}_{\mathbf{1}}\left(\boldsymbol{\mu}_{\mathbf{b}}\right)$ & $\boldsymbol{n}$ & $\boldsymbol{\tau}(\mathbf{s})^{a}$ \\
\hline 2 & 0.46512 & 0.03118 & 0.783 & 97.035 \\
2.5 & 0.48708 & 0.02152 & 0.786 & 92.936 \\
3 & 0.41782 & 0.01605 & 0.786 & 95.310 \\
3.5 & 0.40869 & 0.01375 & 0.780 & 94.482 \\
4 & 0.39878 & 0.01184 & 0.789 & 94.369 \\
4.5 & 0.42105 & 0.01211 & 0.848 & 93.179 \\
5 & 0.40982 & 0.01019 & 0.801 & 88.046 \\
5.5 & 0.33137 & 0.00536 & 0.818 & 86.703
\end{tabular}

${ }^{a}$ Values for $\tau$ are likely only accurate to three significant figures. The full value extracted from the fit is reported for reproducibility.

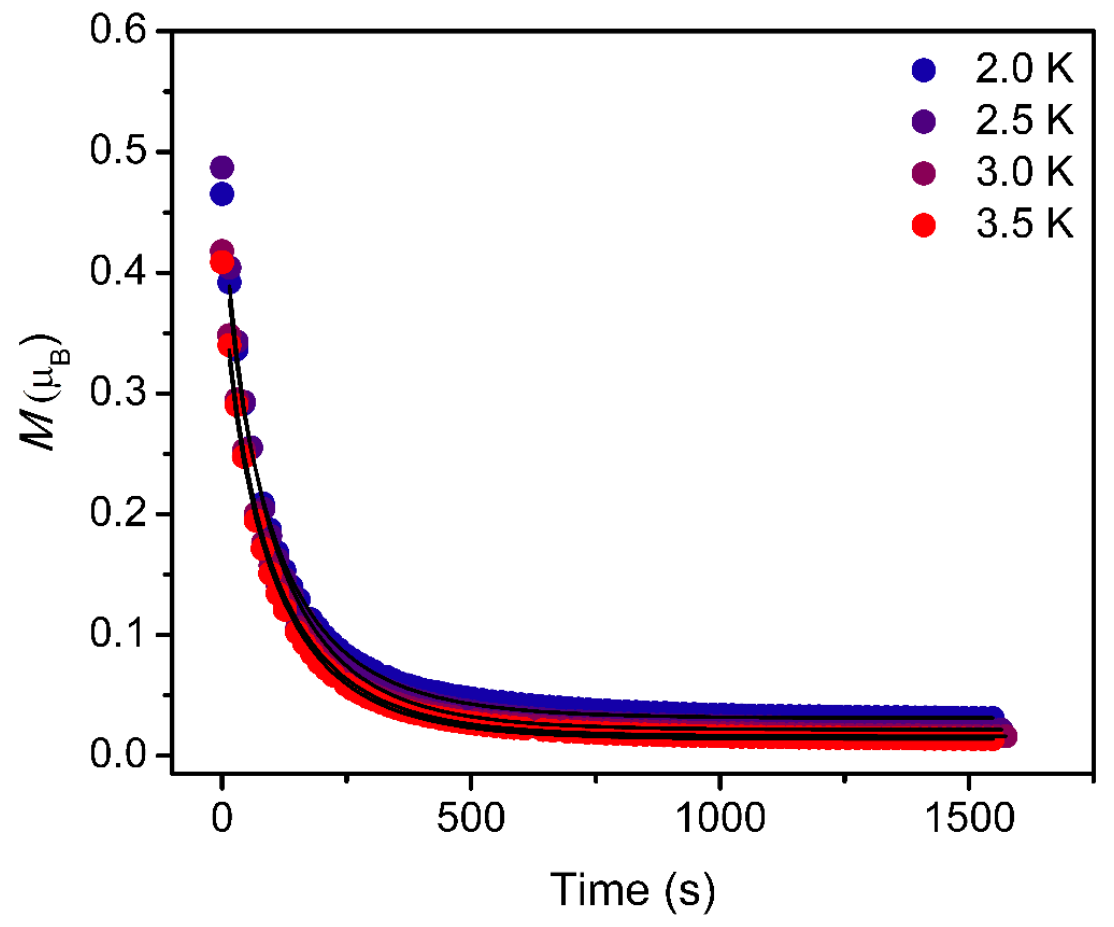

Figure S73. Dc magnetic relaxation data for an $8.0 \mathrm{mM}$ of 4-Dy collected at 2.0, 2.5, 3.0, and 3.5 $\mathrm{K}$ under zero applied magnetic field. Black lines represent the fits to the data using stretched exponential functions, which were used to extract $\tau$. 


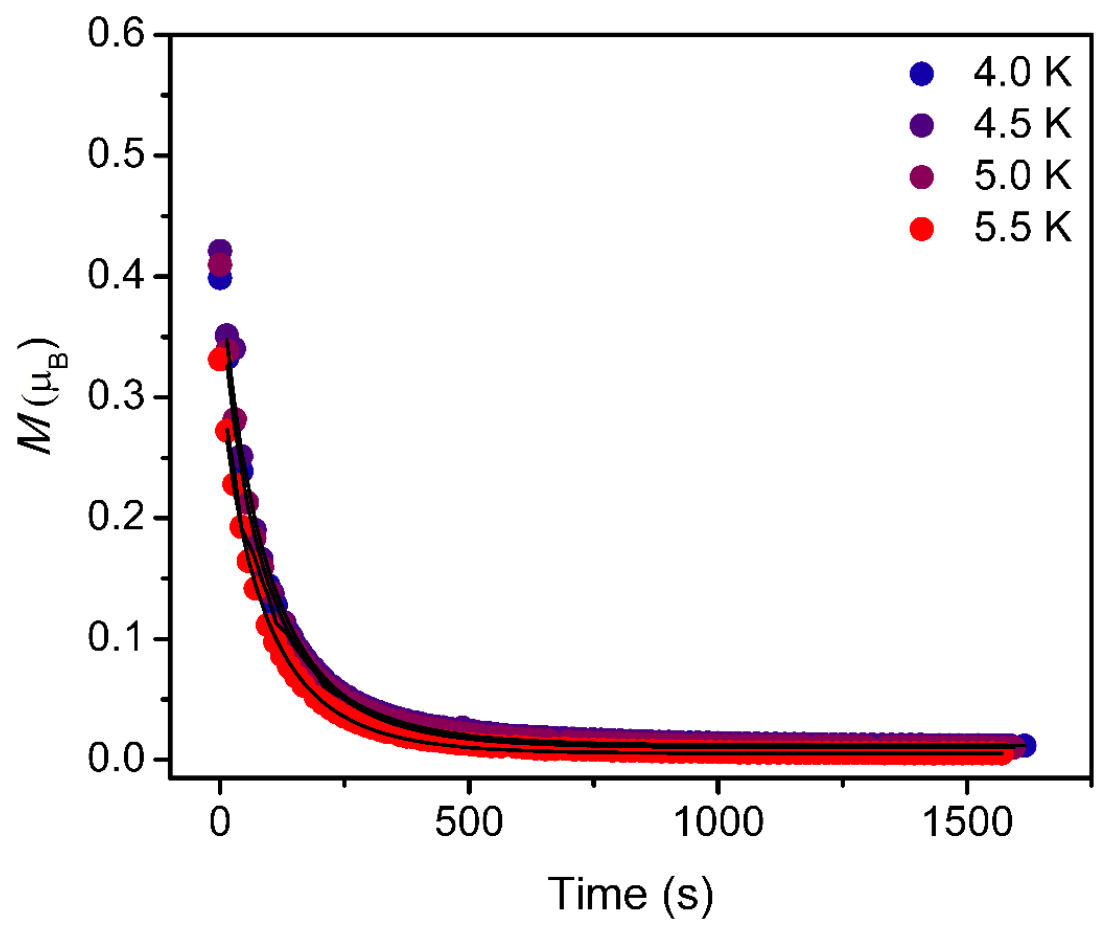

Figure S74. De magnetic relaxation data for an $8.0 \mathrm{mM}$ of 4-Dy collected at 4.0, 4.5, 5.0, and 5.5 $\mathrm{K}$ under zero applied magnetic field. Black lines represent the fits to the data using stretched exponential functions, which were used to extract $\tau$.

Table S17. Parameters used to fit de magnetic relaxation data for an $8.0 \mathrm{mM}$ solution of 4-Dy collected under a 500 Oe field and magnetic relaxation times extracted from these fits.

\begin{tabular}{ccccc}
\hline $\boldsymbol{T}(\mathbf{K})$ & $\boldsymbol{M}_{\mathbf{0}}\left(\boldsymbol{\mu}_{\mathbf{b}}\right)$ & $\boldsymbol{M}_{\mathbf{1}}\left(\boldsymbol{\mu}_{\mathbf{b}}\right)$ & $\boldsymbol{n}$ & $\boldsymbol{\tau}(\mathbf{s})^{a}$ \\
\hline 2 & 2.62304 & 0.85290 & 0.401 & 38450.579 \\
2.5 & 2.48317 & 0.71844 & 0.414 & 29472.631 \\
3 & 2.37864 & 0.62224 & 0.417 & 25918.041 \\
3.5 & 2.29998 & 0.56128 & 0.426 & 21589.703 \\
4 & 2.23117 & 0.51234 & 0.443 & 17179.222 \\
4.5 & 2.16330 & 0.46698 & 0.472 & 12442.685 \\
5 & 2.06905 & 0.42769 & 0.555 & 5434.814 \\
5.5 & 1.84109 & 0.39963 & 0.642 & 1663.431 \\
6 & 1.31069 & 0.40640 & 0.761 & 458.109 \\
\hline
\end{tabular}

${ }^{a}$ Values for $\tau$ are likely only accurate to three significant figures. The full value extracted from the fit is reported for reproducibility. 


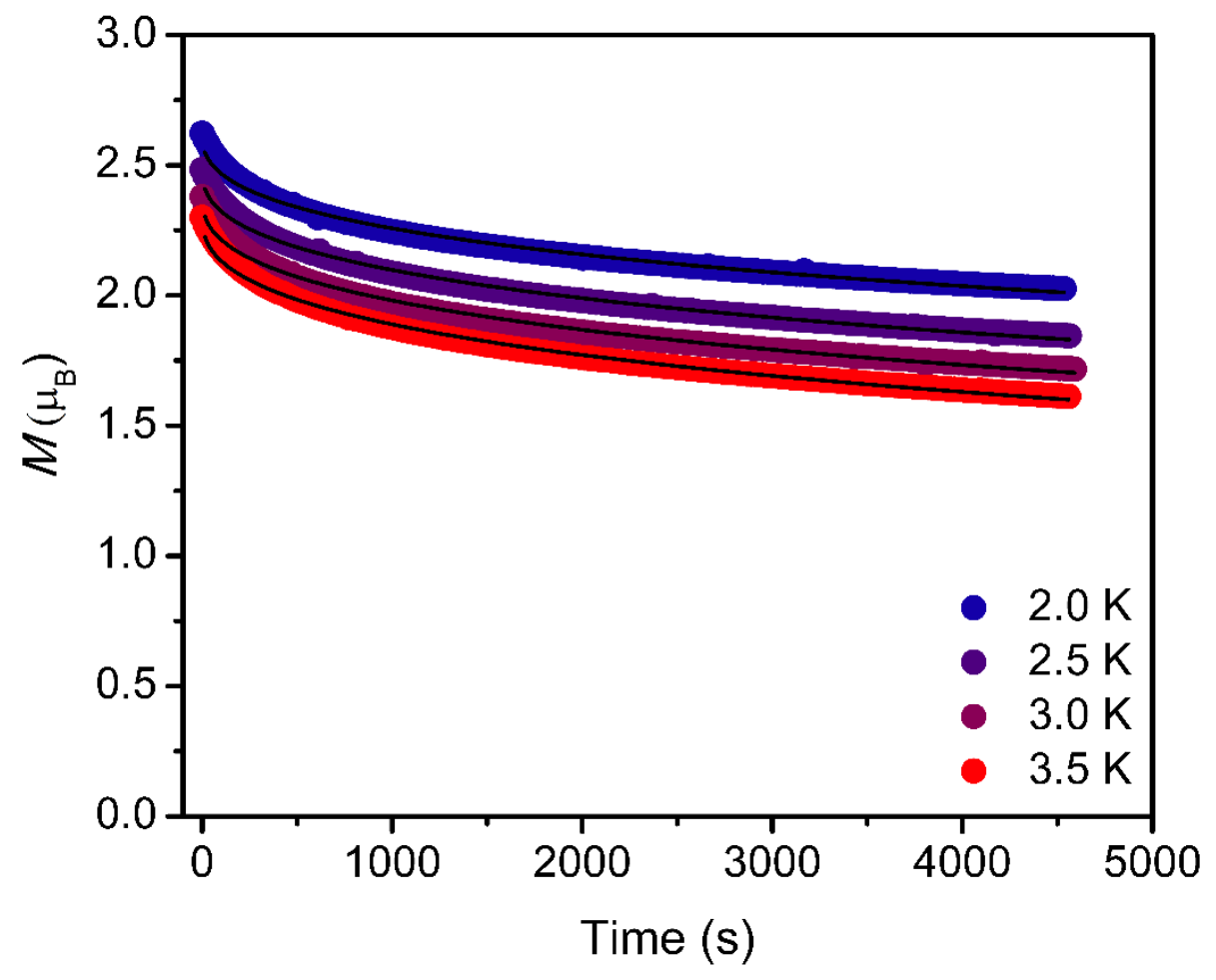

Figure S75. De magnetic relaxation data for an $8.0 \mathrm{mM}$ of 4-Dy collected at 2.0, 2.5, 3.0, and 3.5 $\mathrm{K}$ under a 500 Oe field. Black lines represent the fits to the data using stretched exponential functions, which were used to extract $\tau$.

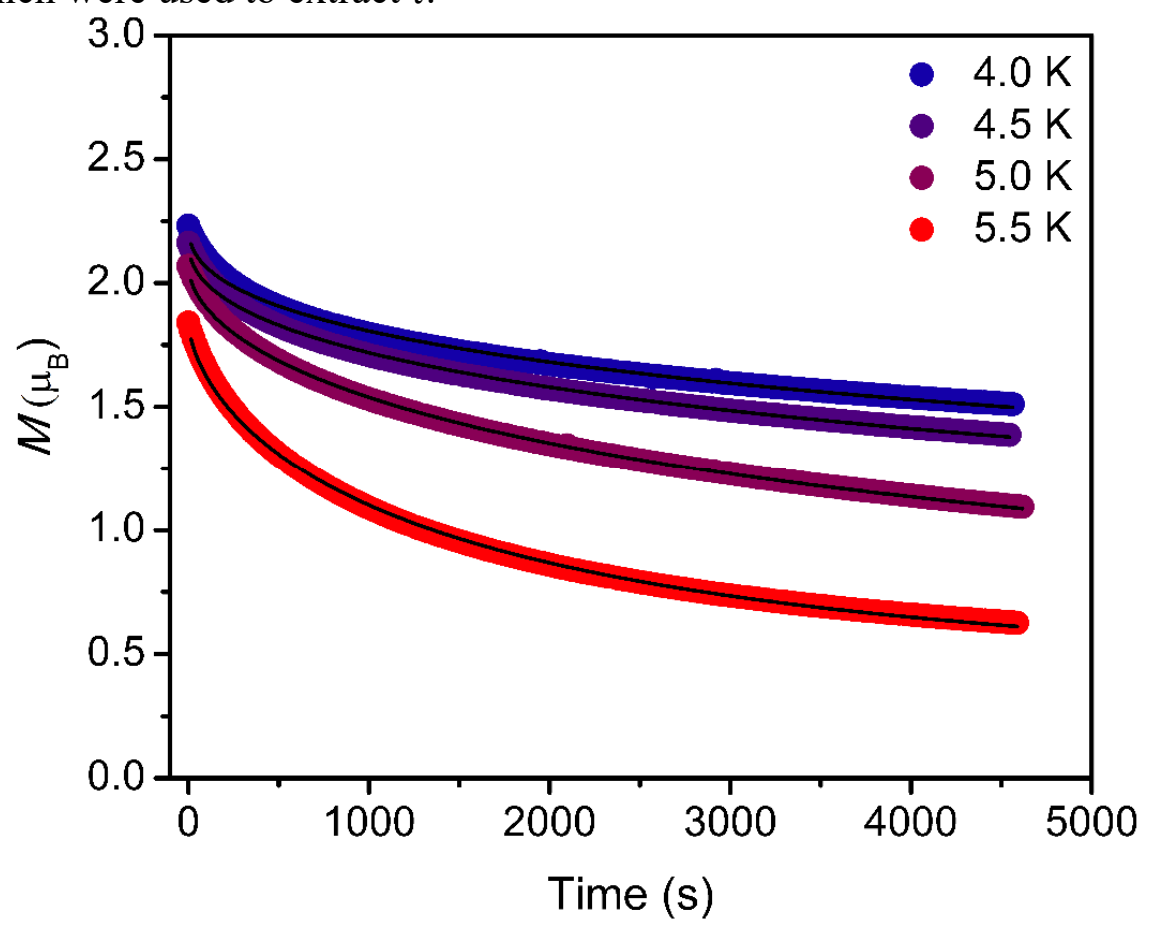

Figure S76. De magnetic relaxation data for an $8.0 \mathrm{mM}$ of 4-Dy collected at 4.0, 4.5, 5.0, and 5.5 $\mathrm{K}$ under an applied magnetic field of 500 Oe. Black lines represent the fits to the data using stretched exponential functions, which were used to extract $\tau$. 


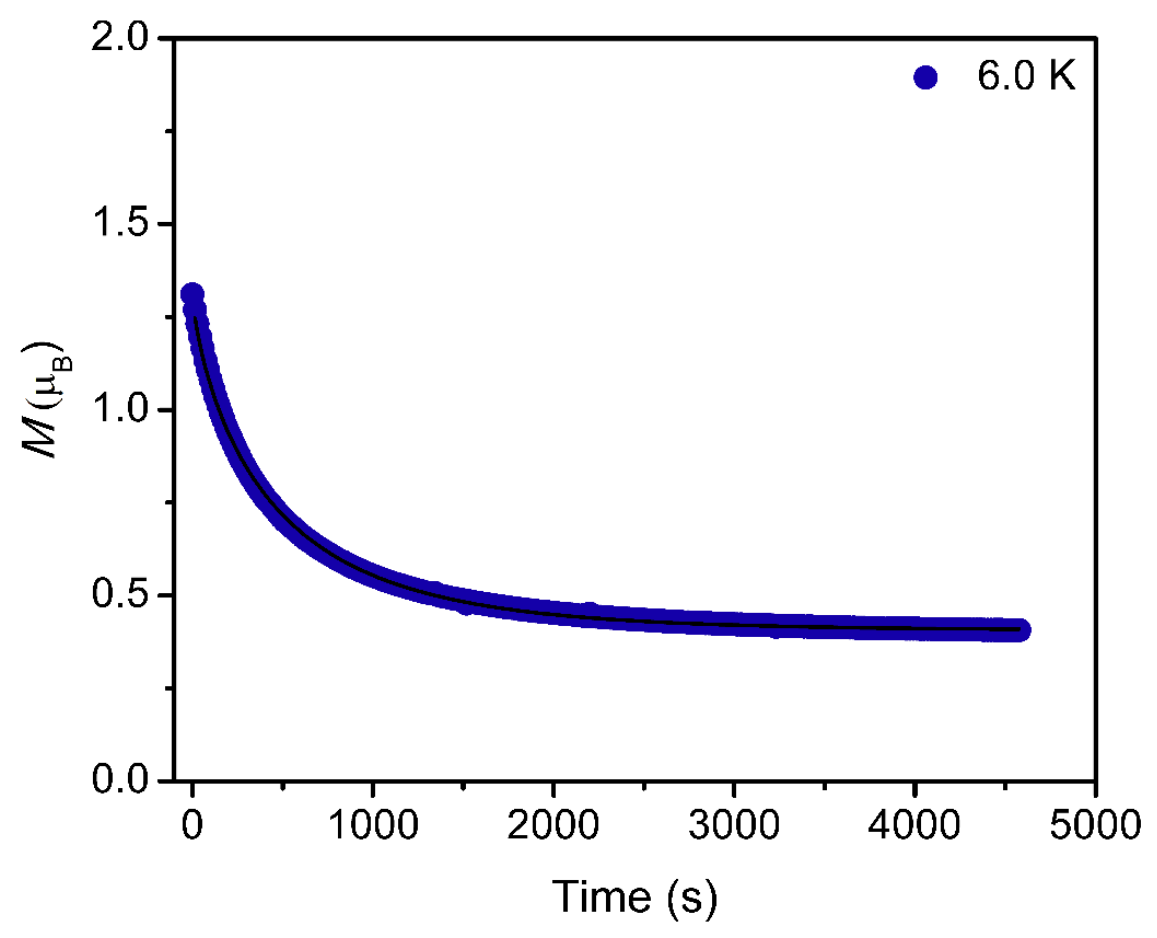

Figure S77. Dc magnetic relaxation data for an $8.0 \mathrm{mM}$ of 4-Dy collected at $6.0 \mathrm{~K}$ under a 500 Oe field. The black line represent the fit to the data using a stretched exponential function, which was used to extract $\tau$.

\section{Section S5.4 Analysis of Magnetic Relaxation Dynamics}

Plots of $\ln (\tau)$ versus $1 / T$ for values extracted from ac susceptibility data for 1-Dy through 4-Dy were linear, indicative of an Orbach relaxation mechanism. Data were thus fit to the equation:

$$
\tau^{-1}=\tau_{0}^{-1} e^{-U_{e f f} / k_{b} T}
$$

where $\tau$ is the magnetic relaxation time, $\tau_{0}$ is the attempt time, $U_{\text {eff }}$ is the thermal barrier to magnetization reversal, $k_{\mathrm{b}}=0.695 \mathrm{~cm}^{-1} \mathrm{~K}^{-1}$, and $T$ is temperature.

The full range of magnetic relaxation times extracted from ac susceptibility and dc magnetic relaxation experiments for 3-Dy and 4-Dy showed a more complex temperature dependence, indicative of multiple magnetic relaxation pathways. Plots of the log of magnetic relaxation time versus $1 / T$ showed an exponential dependence at intermediate temperatures and flattened at low $T$, suggesting the presence of Raman relaxation and/or quantum tunneling of the magnetization. Accordingly, the data were fit to the equation:

$$
\tau^{-1}=\tau_{0}^{-1} e^{-U_{e f f} / k_{b} T}+\tau_{\text {tunnel }}^{-1}+C T^{n}
$$

where $\tau_{\text {tunnel }}$ is the relaxation time for quantum tunneling of the magnetization and $C$ and $n$ are free variables that describe Raman relaxation. To avoid over-parameterization, $\tau_{0}$ and $U_{\text {eff }}$ were fixed to the values extracted from fits to only the ac magnetic susceptibility measurements. All three 
relaxation processes were necessary to obtain fits that satisfactorily reproduced the data for 3-Dy and 4-Dy.

The magnetic relaxation times extracted from ac susceptibility measurements for 1-Tb were independent of temperature, suggesting magnetic relaxation via quantum tunneling. Accordingly, the data were fit to the equation:

$$
\tau^{-1}=\tau_{\text {tunnel }}^{-1}
$$

For 4-Tb, the magnetic relaxation times showed a more complex temperature dependence. Data were fit to the equation:

$$
\tau^{-1}=\tau_{0}^{-1} e^{-U_{e f f} / k_{b} T}+\tau_{\text {tunnel }}^{-1}+C T^{n}
$$

To avoid over-parameterization, $\tau_{0}$ and $U_{\text {eff }}$ were fixed to the values extracted from fits to only the high-temperature linear portion of the ac susceptibility measurements. All three relaxation processes were necessary in order to obtain fits that satisfactorily reproduced the data for 4-Tb.

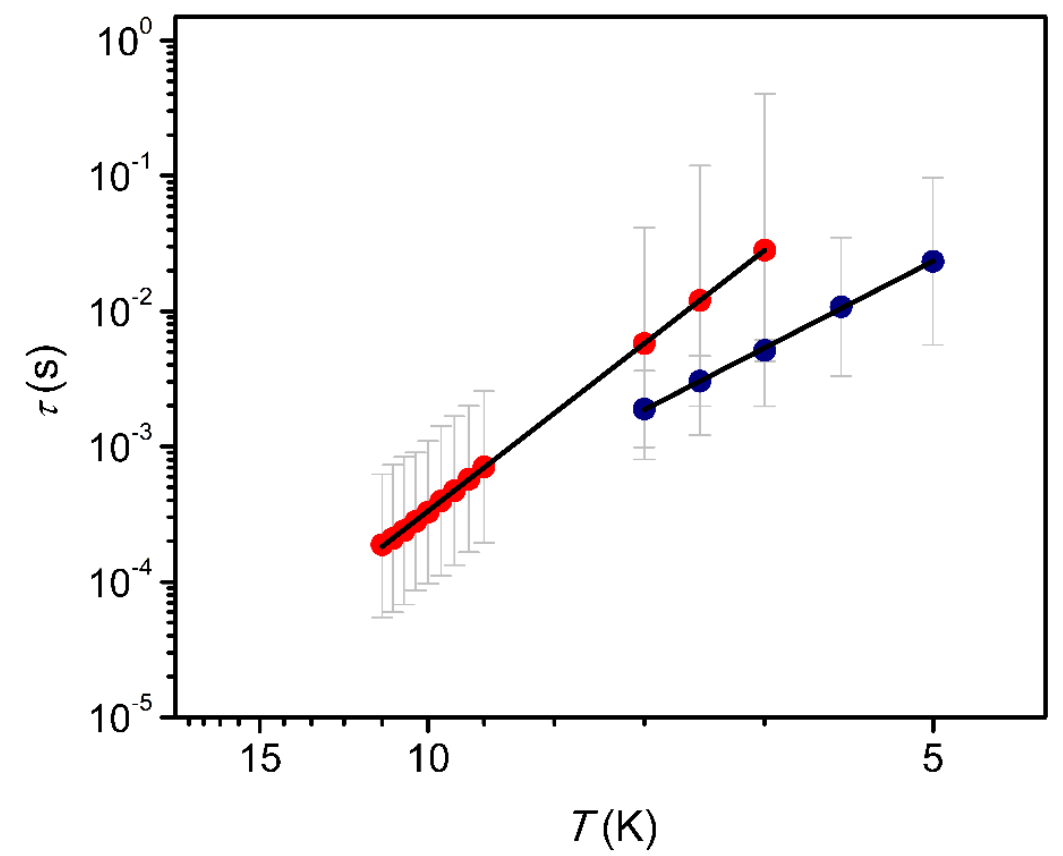

Figure S78. Plot of magnetic relaxation time versus temperature for 1-Dy (dominant process, blue circles; minor process, red circles). Black lines represent fits of the data to an Orbach relaxation processes, which yielded $U_{\text {eff }}=31(13)$ and $46(18) \mathrm{cm}^{-1}$ and $\tau_{0}=10^{-5.5(1.3)}$ and $10^{-6.4(1.2)} \mathrm{s}$ for the dominant and minor processes, respectively. The $\tau_{ \pm}(1 \sigma)$ uncertainty ranges for each $\tau$ value are displayed in light gray. 


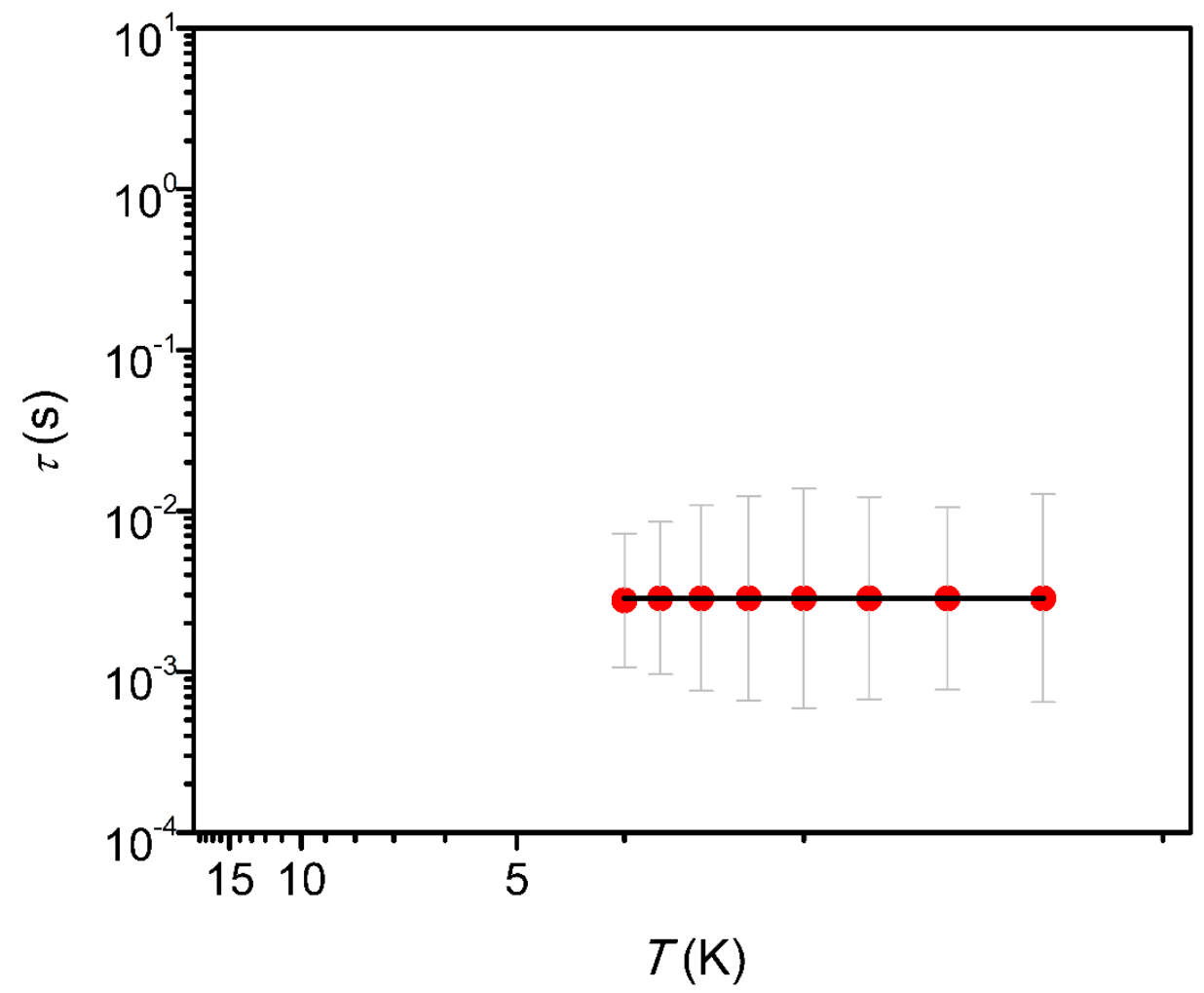

Figure S79. Plot of magnetic relaxation time versus temperature for 1-Tb (ac susceptibility, red circles). The black line represents a fit of the data to a quantum tunneling relaxation process with $\tau_{\text {tunnel }}=10^{-2.5(6)} \mathrm{s}$. The $\tau_{ \pm}(1 \sigma)$ uncertainty ranges for each $\tau$ value are displayed in light gray. 


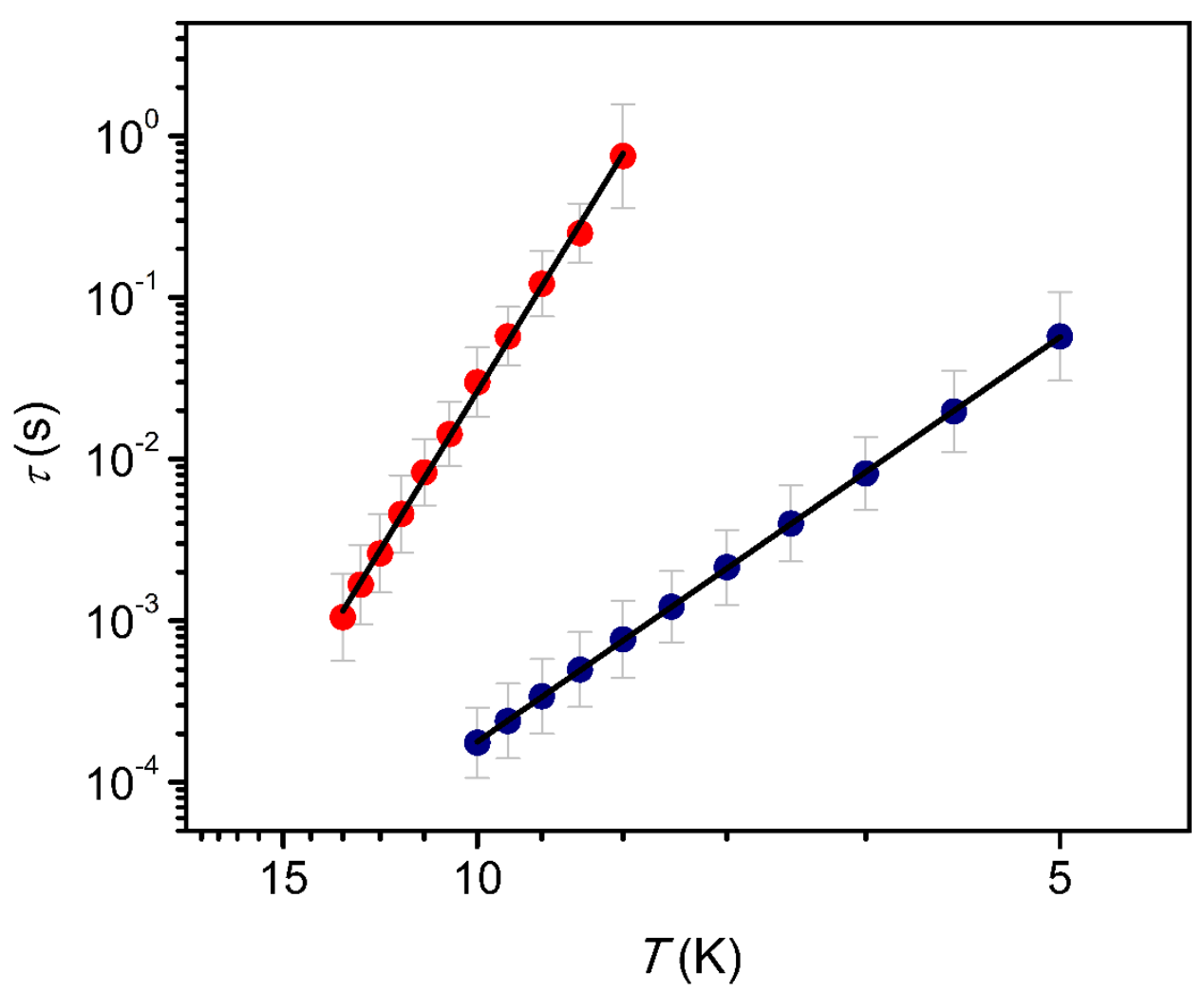

Figure S80. Plot of magnetic relaxation time versus temperature for 2-Dy (dominant process, blue circles; minor process, red circles). Black lines represent fits of the data to Orbach relaxation processes, yielding $U_{\text {eff }}=40(4)$ and $94(10) \mathrm{cm}^{-1}$ and $\tau_{0}=10^{-6.3(4)}$ and $10^{-7.5(6)} \mathrm{s}$ for the dominant and minor processes, respectively. The $\tau_{ \pm}(1 \sigma)$ uncertainty ranges for each $\tau$ value are displayed in light gray. 


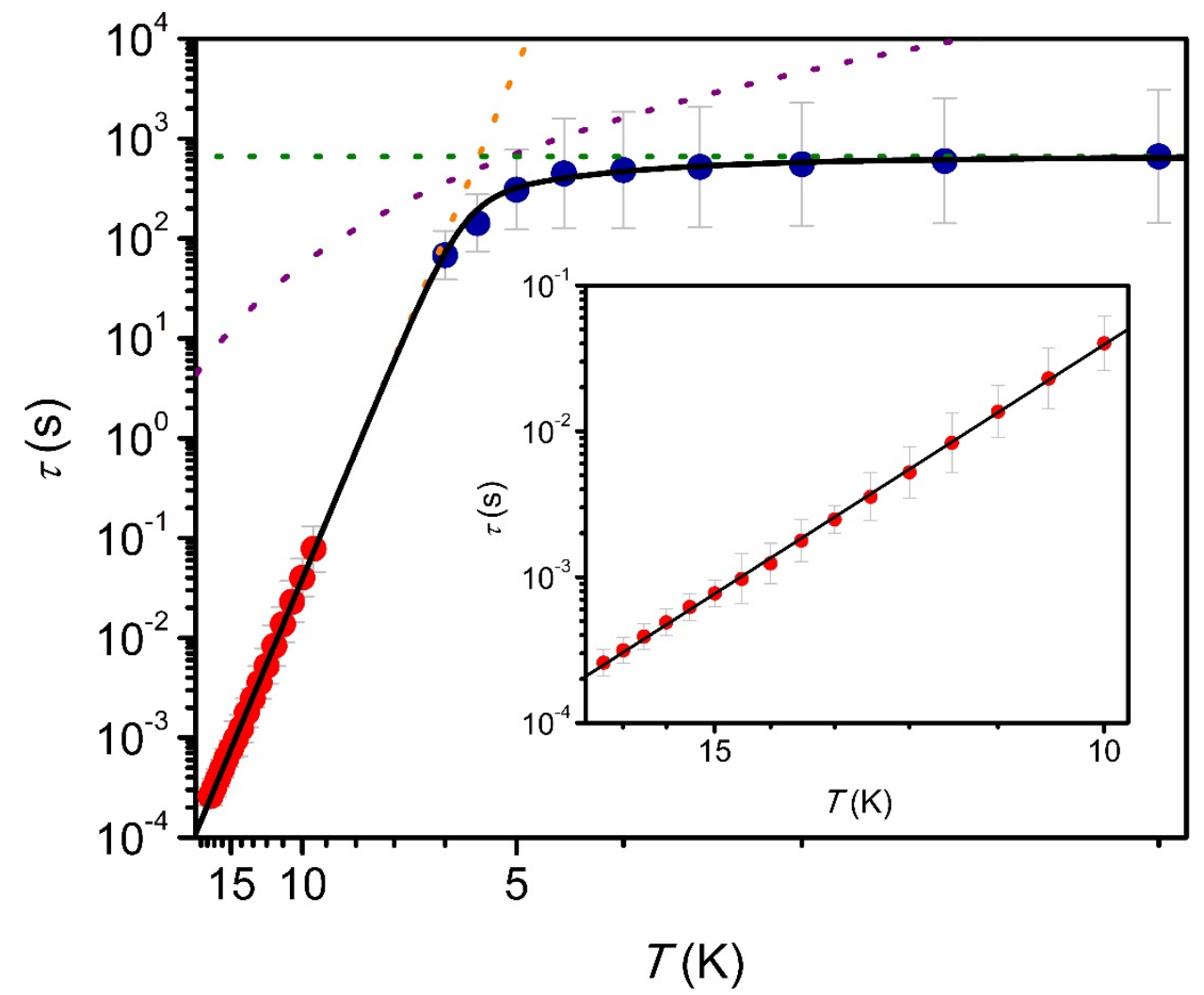

Figure S81. Plot of magnetic relaxation time versus temperature for a solid sample of 3-Dy (dc relaxation, blue circles; ac susceptibility, red circles). Green, purple, and orange lines represent fits to quantum tunneling, Raman, and Orbach relaxation processes, respectively. The black line represents the total fit to the data. The $\tau_{ \pm}(1 \sigma)$ uncertainty ranges for each $\tau$ value are displayed in light gray. 


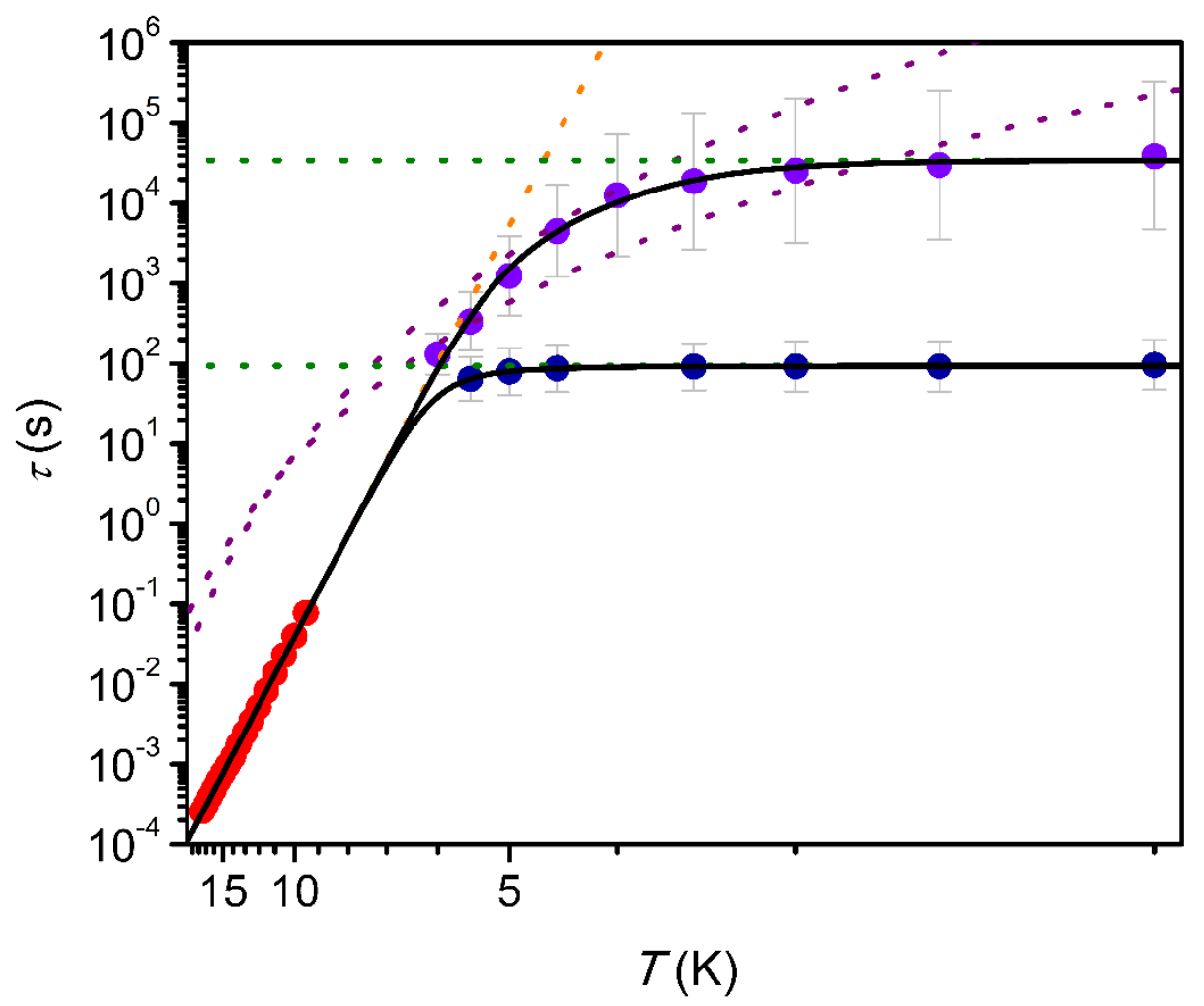

Figure S82. Plot of magnetic relaxation time versus temperature for a $7.8 \mathrm{mM}$ solution of 3-Dy (ac susceptibility for solid sample under zero applied field, red circles; dc relaxation for a $7.8 \mathrm{mM}$ solution under zero applied field, blue circles; de relaxation for a $7.8 \mathrm{mM}$ solution under an applied field of $500 \mathrm{Oe}$, violet circles). Green, purple, and orange lines represent fits to quantum tunneling, Raman, and Orbach relaxation processes, respectively. The black line represents the total fit to the data. The $\tau_{ \pm}(1 \sigma)$ uncertainty ranges for each $\tau$ value are displayed in light gray.

Table S18. Parameters used to fit the Arrhenius plots for 3-Dy.

\begin{tabular}{cccc}
\hline & $\begin{array}{c}\text { Solid Sample } \\
\left(\boldsymbol{H}_{\mathbf{d c}}=\mathbf{0 ~ O e}\right)\end{array}$ & $\begin{array}{c}\mathbf{7 . 8} \mathbf{~ m M ~ S o l u t i o n} \\
\left(\boldsymbol{H}_{\mathbf{d c}}=\mathbf{0} \text { Oe }\right)\end{array}$ & $\begin{array}{c}\mathbf{7 . 8} \mathbf{~ m M ~ S o l u t i o n} \\
\left(\boldsymbol{H}_{\mathbf{d c}}=\mathbf{5 0 0} \text { Oe }\right)\end{array}$ \\
\hline$U_{\text {eff }}\left(\mathrm{cm}^{-1}\right)$ & $82(5)$ & - & - \\
$\tau_{0}(\mathrm{~s})$ & $10^{-6.5(2)}$ & - & - \\
$\tau_{\text {tunnel }}(\mathrm{s})$ & $10^{2.8(7)}$ & $10^{2.0(3)}$ & $10^{4.5(1.0)}$ \\
$C$ & $10^{-5.4(2)}$ & $10^{-7.3(2)}$ & $10^{-9.2(3)}$ \\
$n$ & $3.7(2)$ & $6.5(5)$ & $8.3(8)$ \\
\hline
\end{tabular}




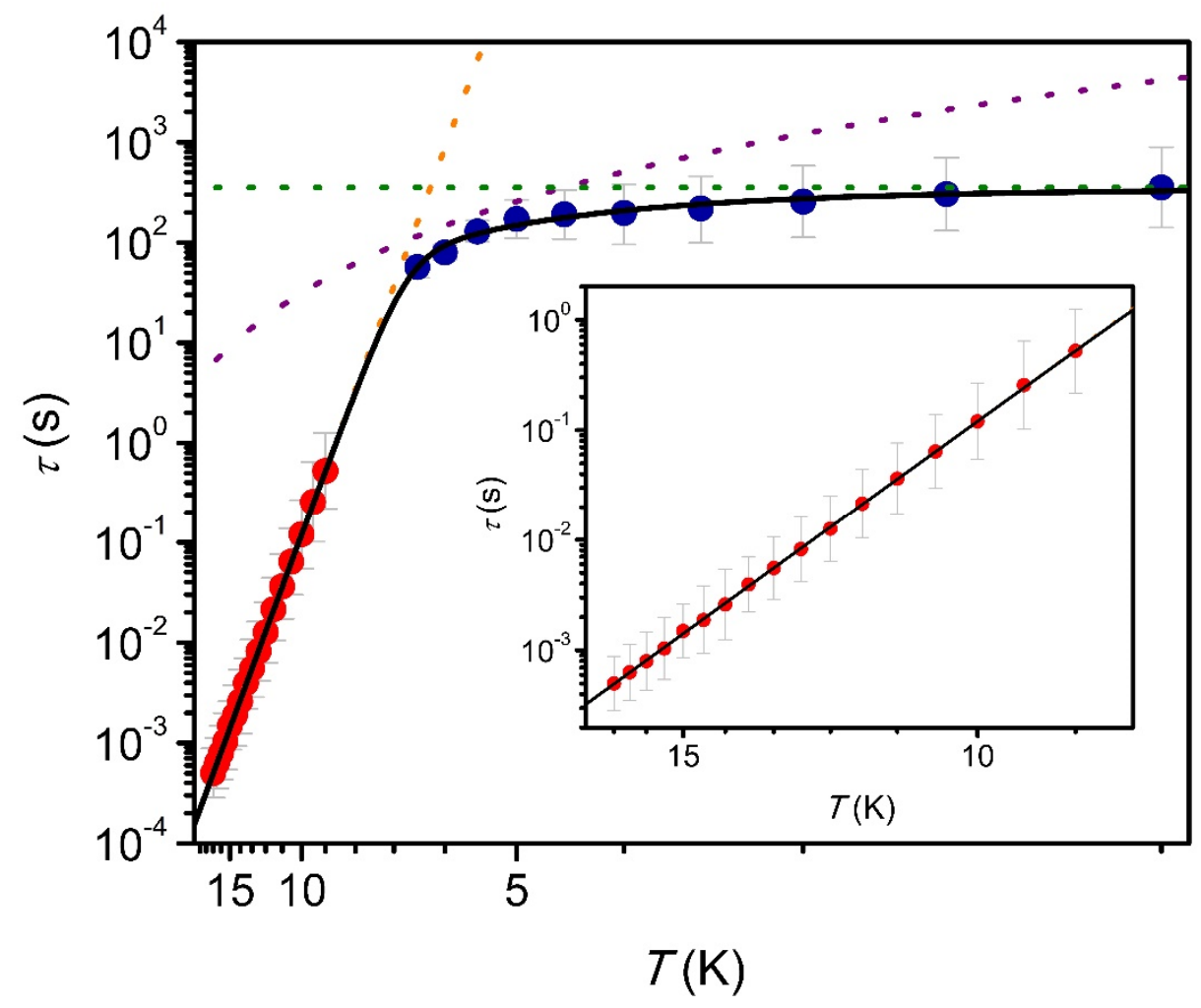

Figure S83. Plot of magnetic relaxation time versus temperature for 4-Dy (dc relaxation, blue circles; ac susceptibility, red circles). Green, purple, and orange lines represent fits to quantum tunneling, Raman, and Orbach relaxation processes, respectively. The black line represents the total fit to the data. The $\tau_{ \pm}(1 \sigma)$ uncertainty ranges for each $\tau$ value are displayed in light gray. 


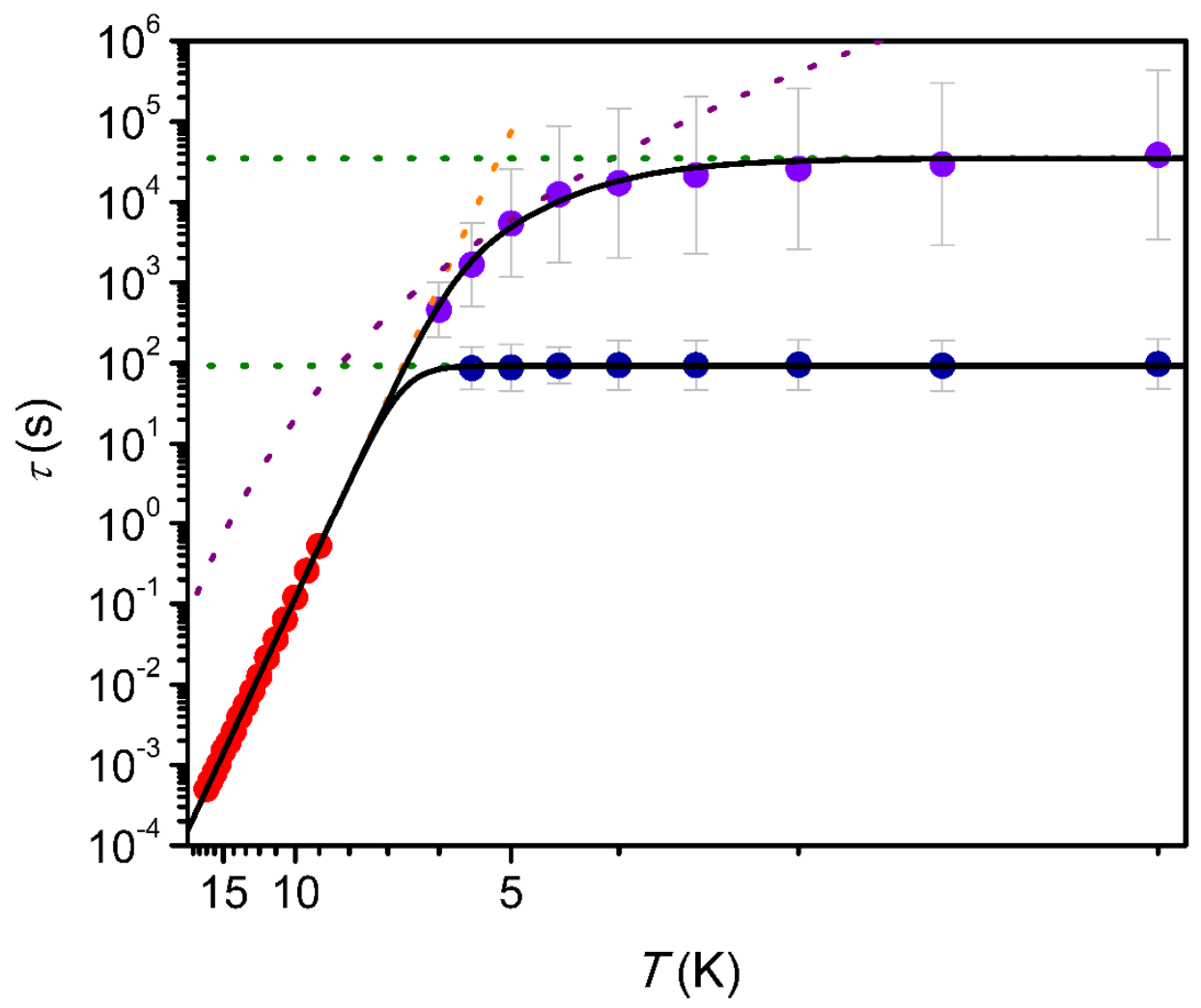

Figure S84. Plot of magnetic relaxation time versus temperature for an $8.0 \mathrm{mM}$ solution of 4-Dy (ac susceptibility for solid sample under zero applied field, red circles; dc relaxation for an $8.0 \mathrm{mM}$ solution under zero applied field, blue circles; dc relaxation for an $8.0 \mathrm{mM}$ solution under an applied field of $500 \mathrm{Oe}$, violet circles). Green, purple, and orange lines represent fits to quantum tunneling, Raman, and Orbach relaxation processes, respectively. The black line represents the total fit to the data. The $\tau_{ \pm}(1 \sigma)$ uncertainty ranges for each $\tau$ value are displayed in light gray.

Table S19. Parameters used to fit the Arrhenius plots for 4-Dy.

\begin{tabular}{cccc}
\hline & $\begin{array}{c}\text { Solid Sample } \\
\left(\boldsymbol{H}_{\mathbf{d c}}=\mathbf{0} \text { Oe }\right)\end{array}$ & $\begin{array}{c}\mathbf{8 . 0} \mathbf{~ m M ~ S o l u t i o n} \\
\left(\boldsymbol{H}_{\mathbf{d c}}=\mathbf{0} \text { Oe }\right)\end{array}$ & $\begin{array}{c}\mathbf{8 . 0} \mathbf{~ m M ~ S o l u t i o n} \\
\left(\boldsymbol{H}_{\mathbf{d c}}=\mathbf{5 0 0} \text { Oe }\right)\end{array}$ \\
\hline$U_{\text {eff }}\left(\mathrm{cm}^{-1}\right)$ & $93(9)$ & - & - \\
$\tau_{0}(\mathrm{~s})$ & $10^{-6.7(5)}$ & - & - \\
$\tau_{\text {tunnel }}(\mathrm{s})$ & $10^{2.5(4)}$ & $10^{2.0(3)}$ & $10^{4.5(1.1)}$ \\
$C$ & $10^{-4.5(2)}$ & - & $10^{-9.5(6)}$ \\
$n$ & $3.0(2)$ & - & $8.2(1)$ \\
\hline
\end{tabular}




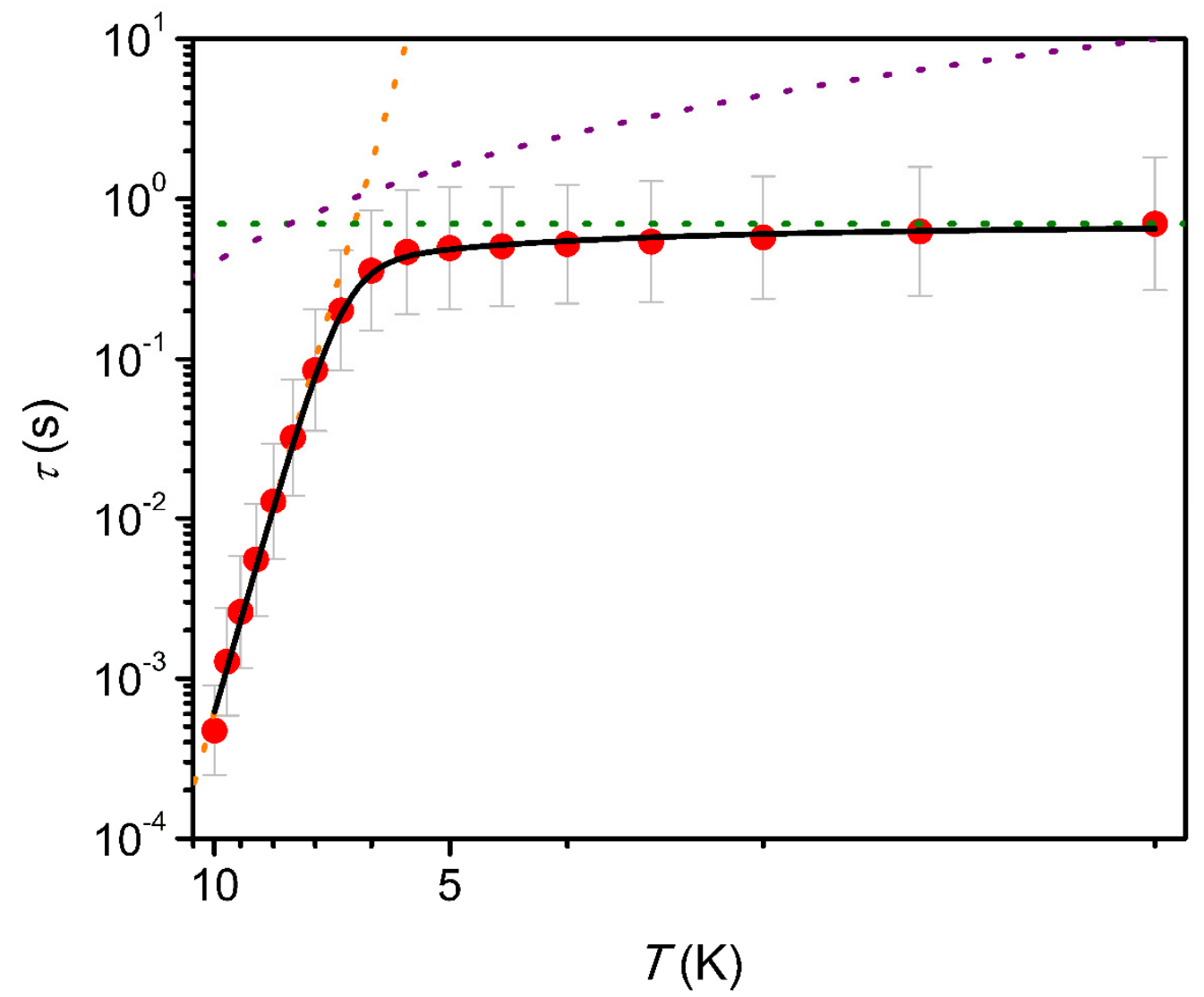

Figure S85. Plot of magnetic relaxation time versus temperature for 4-Tb (ac susceptibility, red circles). Green, purple, and orange lines represent fits to quantum tunneling, Raman, and Orbach relaxation processes, respectively. The black line represents the total fit to the data, which yielded $U_{\text {eff }}=82(13) \mathrm{cm}^{-1}, \tau_{0}=10^{-8.3(1.0)} \mathrm{s}, \tau_{\text {tunnel }}=10^{-0.15(41)} \mathrm{s}, C=10^{-1.6(3)}$, and $n=2.0(1)$. The $\tau_{ \pm}(1 \sigma)$ uncertainty ranges for each $\tau$ value are displayed in light gray.

\section{Section S5.5 Empirical Correlation Between $\boldsymbol{U}_{\text {eff }}$ and $\boldsymbol{J}_{\mathrm{Gd}-\mathrm{rad}}$}

The energy of electronic excited states in a high-anisotropy, strongly-coupled lanthanide complex can be predicted according to the Ising model, which relates energy differences to $J_{\mathrm{Ln} \text {-rad }}$ and the magnitude of $\Delta J$ resulting from a spin flip. For instance, the energy difference between the ground and first excited state for a radical-bridged dysprosium complex that follows the Ising model is equal to $15 \mid J_{\text {Dy-rad }}$ (Figure 3, left). In radical-bridged lanthanide complexes, it has been shown that the value of $U_{\text {eff }}$ typically corresponds to the energy difference between the ground and first excited exchange-coupled states. ${ }^{3,4}$ Assuming that the coupling constant for a given Dy complex $\left(J_{\text {Dy-rad }}\right)$ is proportional to the coupling constant of the $\mathrm{Gd}$ congener $\left(J_{\mathrm{Gd}-\mathrm{rad}}\right)$ and that the proportionality constant which relates $J_{\text {Dy-rad }}$ and $J_{\mathrm{Gd}-\text { rad }}$ is similar for analogous complexes, such as multinuclear lanthanide complexes bridged by organic radicals, we propose the empirical relationship $U_{\text {eff }}=$ $C \mid J_{\mathrm{Gd}}$-rad $\mid$ can be used to predict the magnitude of the barrier to magnetic relaxation for Dy systems. This relationship would give rise to a linear plot of $U_{\text {eff }}$ versus $J_{\text {Gd-rad }}$ for a given series of compounds that display high single-ion anisotropy. 
If a radical-bridged lanthanide complex displays low single-ion anisotropy, it will deviate from the Ising model. Mixing of excited Kramers doublets with the ground doublet will lead to a lower value of $U_{\text {eff }}$ relative to an analogous system with large single-ion anisotropy. In this scenario, the value of $U_{\text {eff }}$ will be dictated both by the value of $J_{\text {Dy-rad }}$ and the relative splitting of crystal field states on the $\mathrm{Dy}^{3+}$ ions (Figure 3, right). As such, a plot of $U_{\text {eff versus }} J_{\mathrm{Gd}-\text { rad }}$ is not expected to be linear.

Figure 4 in the main text is a plot of the experimental $U_{\text {eff }}$ value for the dominant magnetic relaxation process in 1-Dy through 4-Dy versus $\left|J_{\mathrm{Gd}-\mathrm{rad}}\right|$ of the corresponding Gd derivative. Here, the plotted value of $\left|J_{\mathrm{Gd}-\mathrm{rad}}\right|$ is an average of the values extracted from fits to dc susceptibility data collected at 1, 5, and $10 \mathrm{kOe}$ for each compound, as described above. The plot in Figure 4 demonstrates that $U_{\text {eff }}$ indeed correlates linearly with $\left|J_{\mathrm{Gd}-\mathrm{rad}}\right|$ for 1-Dy through 4-Dy, with a bestfit line of $U_{\text {eff }}=7.617\left|J_{\mathrm{Gd}-\mathrm{rad}}\right|+9.582\left(\mathrm{R}^{2}=0.9997\right)$. The non-zero value of the $y$-intercept is likely meaningless, as this relationship is not expected to apply to systems with small values of $J_{\mathrm{Gd}-\mathrm{rad}}$, for which single-ion effects are expected to be dominant. The linear correlation observed for this data suggests that 1-Dy through 4-Dy can be accurately described by the Ising model.

In order to test the generality of this empirical model, we plotted the data for all dysprosium complexes that contain an open-shell organic ligand for which both $U_{\text {eff }}$ of the complex and $J_{\mathrm{Gd}-}$ rad of the gadolinium analogue have been determined (see Table S20 below). ${ }^{3}$ Gratifyingly, the best-fit line for 1-Dy through 4-Dy accurately models several previously reported high-anisotropy multinuclear lanthanide complexes bridged by organic radicals (see main text for discussion).

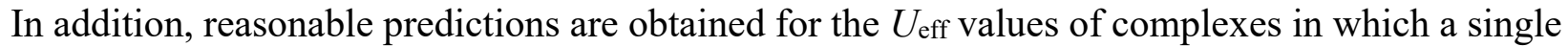
$\mathrm{Dy}^{3+}$ ion interacts with an organic radical. Experimental $U_{\text {eff }}$ values for these complexes are likely lower than the predicted values due to low single-ion anisotropy. Indeed, all of these complexes feature $\mathrm{Dy}^{3+}$ ions with high coordination numbers $(\geq 7)$, which limits anisotropy.

The empirical correlation derived for 1-Dy through 4-Dy offers insight into how the $U_{\text {eff }}$ value of a particular organic radical-bridged dysprosium complex can be increased. If a complex falls along the best-fit line, it displays the maximal value of $U_{\text {eff }}$ that is possible given its value of $J_{\text {Gd-rad. In }}$

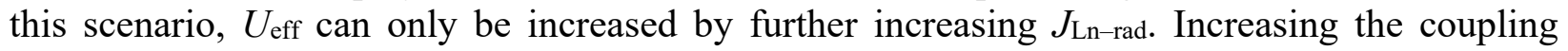
strength should result in diagonal movement along the best-fit line towards the top right corner of the graph. Increasing single-ion anisotropy will not lead to a larger energy difference between the ground and first excited state ( $\left.U_{\text {eff }}\right)$ for these molecules.

If a complex falls below the best-fit line, the value of $U_{\text {eff }}$ can be increased by maximizing singleion anisotropy. This will correspond to vertical movement along the graph towards the best-fit line, which represents the maximal value of the energetic splitting between the ground and first excited state $\left(U_{\text {eff }}\right)$ for that complex given the strength of magnetic exchange coupling. Increasing the value of $J_{\mathrm{Ln} \text {-rad }}$ may increase the value of $U_{\text {eff }}$ somewhat, but the relationship between the two variables is hard to predict, as such complexes do not adhere to an Ising model.

The energy difference between the ground and first excited state for a radical-bridged dysprosium complex that follows the Ising model is equal to $15\left|J_{\text {Dy-rad }}\right|$. Thus, the magnitude of $J_{\text {Dy-rad }}$ for complexes which fall along the best fit line in the empirical correlation can be estimated using the experimental $U_{\text {eff }}$ value. According to this analysis, $J_{\text {Dy-rad }}=-2.1,-2.7,-5.5$, and $-6.2 \mathrm{~cm}^{-1}$ for 1-Dy through 4-Dy, respectively. Estimates for $J_{\text {Dy-rad }}$ determined in this manner could potentially be used to benchmark future computational studies on organic radical-bridged dysprosium complexes. 
Table S20. Experimental $U_{\text {eff }}$ values $\left(\mathrm{cm}^{-1}\right)$ for radical-containing dysprosium complexes and $J_{\mathrm{Gd}-}$ rad $\left(\mathrm{cm}^{-1}\right)$ for the corresponding gadolinium derivative, which were used to construct Figure 4 in the main text. The predicted value of $U_{\text {eff }}$ according to the empirical model derived for 1-Dy through 4-Dy is also included.

\begin{tabular}{|c|c|c|c|c|}
\hline Complex & $\begin{array}{l}J_{\text {Gd-rad }} \\
\left(\mathrm{cm}^{-1}\right) \\
\end{array}$ & $\begin{array}{c}\text { Experimental } \\
U_{\text {eff }}\left(\mathrm{cm}^{-1}\right) \\
\end{array}$ & $\begin{array}{l}\text { Predicted } \\
U_{\text {eff }}\left(\mathrm{cm}^{-1}\right) \\
\end{array}$ & Reference \\
\hline 1-Dy & $-2.88^{a}$ & 31 & 32 & This work \\
\hline 2-Dy & $-3.91^{a}$ & 40 & 39 & This work \\
\hline 3-Dy & $-9.53^{a}$ & 82 & 82 & This work \\
\hline 4-Dy & $-10.9^{a}$ & 93 & 93 & This work \\
\hline$\left[\left(\mathrm{Cp}_{2}{ }_{2} \mathrm{Dy}\right)_{2}(\mu-\mathrm{bpym})\right]^{-}$ & -10 & 88 & 86 & 5 \\
\hline$\left(\mathrm{Cp}^{*}{ }_{2} \mathrm{Dy}\right)_{3}(\mu-\mathrm{HAN})$ & -5.0 & 51 & 48 & 6 \\
\hline$\left[\left((\mathrm{HBpz} 3)_{2} \mathrm{Dy}\right)_{2}(\mu-\mathrm{CA})\right]^{-}$ & -2.09 & 22 & 25 & 7 \\
\hline$\left[\left(\mathrm{Cp}^{*}{ }_{2} \mathrm{Dy}\right)_{2}(\mu-\mathrm{tppz})\right]^{+}$ & -6.9 & 36 & 62 & 8 \\
\hline$\left[\left(\mathrm{Cp}{ }_{2} \mathrm{Dy}\right)_{2}(\mu-\mathrm{tppz})\right]^{-}$ & -6.3 & $\mathrm{n} / \mathrm{a}$ & 58 & 8 \\
\hline$\left[\left(\mathrm{Cp}_{2}{ }_{2} \mathrm{Dy}\right)_{2}(\mu \text {-ind })\right]^{-}$ & -11 & 35 & 93 & 9 \\
\hline$\left[\left(\mathrm{Cp}^{*}{ }_{2} \mathrm{Dy}\right)_{2}\left(\mu-\mathrm{N}_{2}\right)\right]^{-}$ & -20 & 123 & 162 & 10 \\
\hline$\left[\left(\left\{\mathrm{N}\left(\mathrm{SiMe}_{3}\right)_{2}\right\}(\mathrm{THF})_{2} \mathrm{Dy}\right)_{2}\left(\mu-\mathrm{N}_{2}\right)\right]^{-}$ & -27 & 108 & 215 & 11 \\
\hline$\left[\mathrm{Dy}(\mathrm{hfac})_{3}(\mathrm{NIT} 4 \mathrm{Py})\right]_{2}$ & 0.89 & 10 & 16 & 12,13 \\
\hline$\left[\mathrm{Dy}(\mathrm{acac})_{3}(\mathrm{NIT} 2 \mathrm{Py})\right] \cdot 0.5 \mathrm{NIT}^{o} \mathrm{Py}$ & 2.23 & 15 & 26 & 14 \\
\hline$\left[\mathrm{Dy}(\mathrm{Phtfac})_{3}(\mathrm{NIT} 4 \mathrm{Py})\right]_{2}$ & 1.84 & 14 & 24 & 15 \\
\hline$\left[\mathrm{Dy}(\mathrm{hfac})_{3}(\mathrm{NITPhO})\right]_{2}$ & 2.75 & 5.2 & 30.5 & 16,17 \\
\hline Dy(tfa) ${ }_{3}(\mathrm{NITBzImH})$ & 0.99 & 3.2 & 17 & 18 \\
\hline
\end{tabular}

${ }^{a}$ Average of values obtained for fits to $0.1,0.5$, and $1 \mathrm{~T}$ data. 


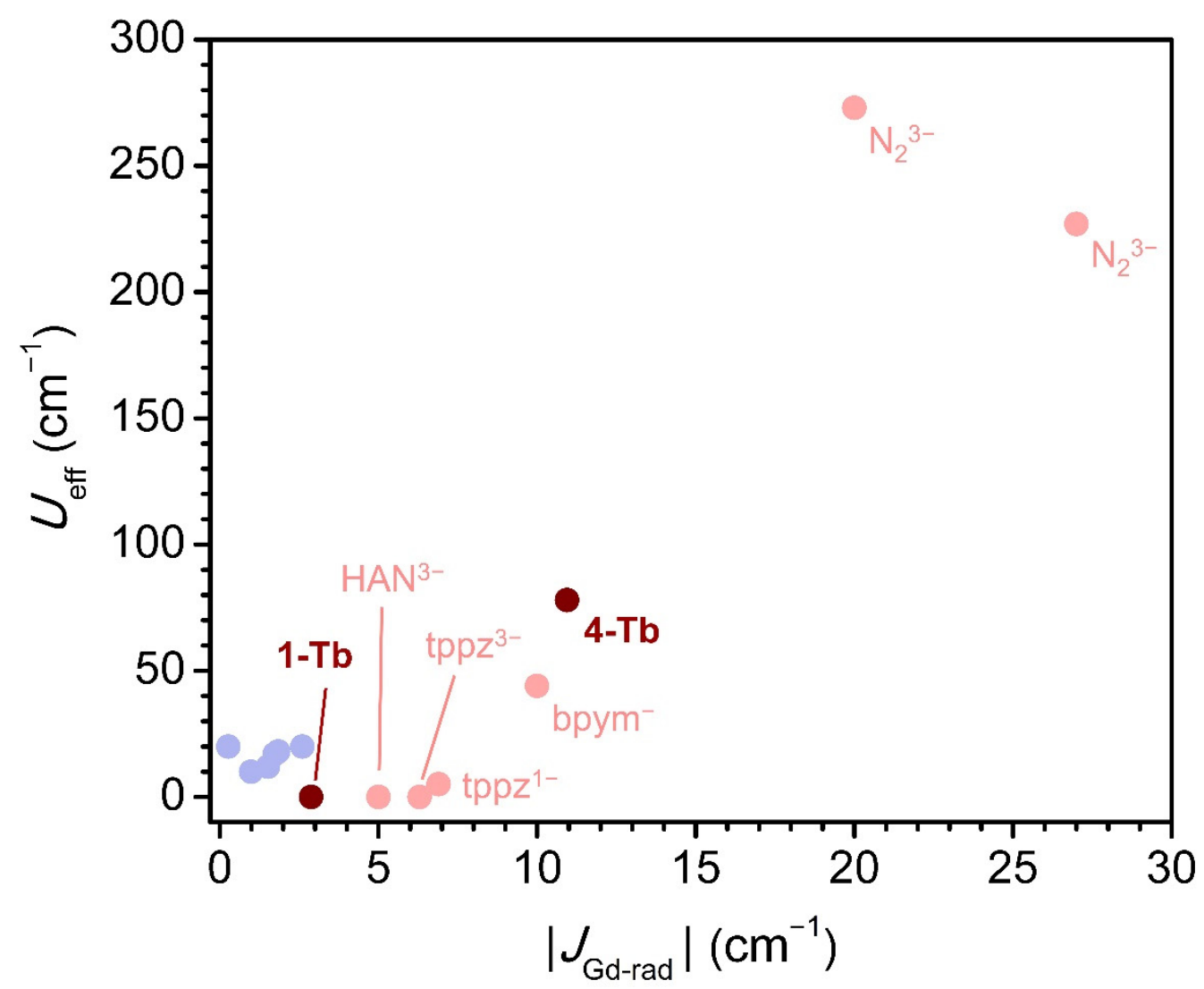

Figure S86. Plot of $U_{\text {eff }}$ values for radical-containing terbium complexes versus $\left|J_{\text {Gd-rad }}\right|$ for the corresponding gadolinium derivative. 1-Tb and 4-Tb are plotted as red circles, radical-bridged complexes are plotted as pink circles, and radical-containing complexes are potted as blue circles.

A plot of the experimental $U_{\text {eff }}$ value for 4-Tb and other radical-bridged terbium complexes in the literature versus $\left|J_{\mathrm{Gd}-\mathrm{rad}}\right|$ for the corresponding gadolinium derivative (Figure S86) shows no discernable linear correlation. This implies that these complexes are not well-described by the Ising model. The low single-ion anisotropy in $\mathbf{1 - T b}, \mathbf{4 - T b}$, and $\left[\left(\mathrm{Cp}_{2}^{*} \mathrm{~Tb}\right)_{2}(\mu-b p y m)\right]^{-}$is corroborated by fast quantum tunneling of the magnetization observed in measurements of the magnetic relaxation dynamics and magnetic hysteresis loops that are closed at zero field. 
Table S21. Experimental $U_{\text {eff values }}\left(\mathrm{cm}^{-1}\right)$ for radical-containing terbium complexes and $J_{\mathrm{Gd}-\mathrm{rad}}$ $\left(\mathrm{cm}^{-1}\right)$ for the corresponding gadolinium derivative, which were used to construct Figure S86.

\begin{tabular}{|c|c|c|c|}
\hline Complex & $J_{\mathrm{Gd}-\mathrm{rad}}\left(\mathrm{cm}^{-1}\right)$ & $\begin{array}{c}\text { Experimental } \\
U_{\text {eff }}\left(\mathbf{c m}^{-1}\right) \\
\end{array}$ & Reference \\
\hline 1-Tb & $-2.88^{a}$ & $\mathrm{n} / \mathrm{a}$ & This work \\
\hline 4-Tb & $-10.9^{a}$ & 79 & This work \\
\hline$\left[\left(\mathrm{Cp}_{2}{ }_{2} \mathrm{~Tb}\right)_{2}(\mu-\mathrm{bpym})\right]^{-}$ & -10 & 44 & 5 \\
\hline$\left(\mathrm{Cp}{ }_{2} \mathrm{~Tb}\right)_{3}(\mu-\mathrm{HAN})$ & -5.0 & $\mathrm{n} / \mathrm{a}$ & 6 \\
\hline$\left[\left(\mathrm{Cp}^{*}{ }_{2} \mathrm{~Tb}\right) 2(\mu-\mathrm{tppz})\right]^{+}$ & -6.9 & 5.1 & 8 \\
\hline$\left[\left(\mathrm{Cp}^{*}{ }_{2} \mathrm{~Tb}\right)_{2}(\mu-\mathrm{tppz})\right]^{-}$ & -6.3 & $\mathrm{n} / \mathrm{a}$ & 8 \\
\hline$\left[\left(\mathrm{Cp}^{*}{ }_{2} \mathrm{~Tb}\right)_{2}\left(\mu-\mathrm{N}_{2}\right)\right]^{-}$ & -20 & 273 & 10 \\
\hline$\left[\left(\left\{\mathrm{N}\left(\mathrm{SiMe}_{3}\right)_{2}\right\}(\mathrm{THF})_{2} \mathrm{~Tb}\right)_{2}\left(\mu-\mathrm{N}_{2}\right)\right]^{-}$ & -27 & 227 & 19 \\
\hline $\mathrm{Tb}(\mathrm{hfac})_{3}(\mathrm{NITPhOEt})_{2}$ & 0.27 & 20 & 20,21 \\
\hline $\mathrm{Tb}(\mathrm{tfa})_{3}(\mathrm{NITBzImH})$ & 0.99 & 10 & 18 \\
\hline$\left[\mathrm{Tb}(\mathrm{hfac})_{3}(\mathrm{NIT} 2 \mathrm{Py})\right] \cdot 0.5 \mathrm{C}_{7} \mathrm{H}_{16}$ & 1.52 & 12 & 22,23 \\
\hline$\left[\mathrm{Tb}(\mathrm{hfac})_{3}\left(\mathrm{NITPhPO}(\mathrm{OEt})_{2}\right)\right]_{2}$ & 1.73 & 17 & 24 \\
\hline$\left[\mathrm{Tb}(\mathrm{Phtfac})_{3}(\mathrm{NIT} 4 \mathrm{Py})\right]_{2}$ & 1.84 & 18 & 15 \\
\hline$\left[\mathrm{Tb}(\mathrm{hfac})_{3}(\mathrm{NIT}-5-\mathrm{Py}-3 \mathrm{Br})\right]_{2}$ & 2.6 & 20 & 25,26 \\
\hline
\end{tabular}

${ }^{a}$ Average of values obtained for fits to $0.1,0.5$, and $1 \mathrm{~T}$ data. 


\section{Section S5.6 Magnetic Hysteresis Data}

Magnetic hysteresis measurements were collected on 1-Dy through 4-Dy, 1-Tb, and 4-Tb at a sweep rate of 82(2) Oe/s for $|H|>10 \mathrm{kOe}$ and 24(1) Oe/s for $|H|<10 \mathrm{kOe}$. Hysteresis measurements were also conducted on a $7.8 \mathrm{mM}$ solution of 3-Dy and an $8.0 \mathrm{mM}$ solution of 4Dy in order to eliminate the influence of molecular dipolar interactions.

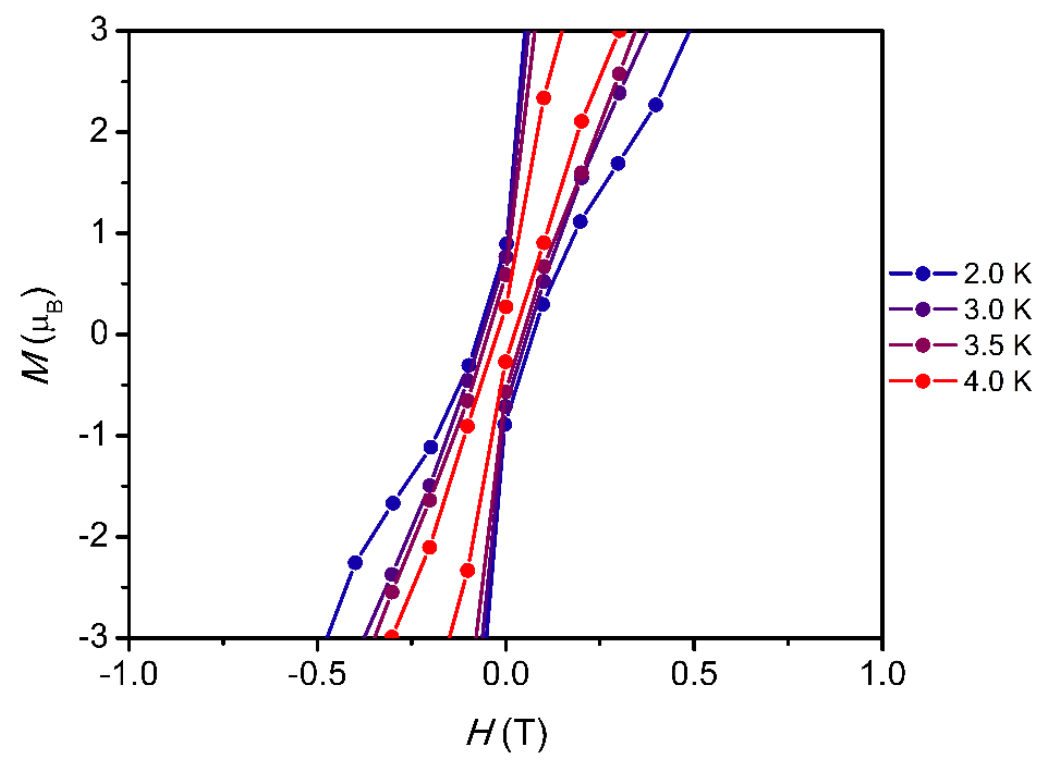

Figure S87. Magnetic hysteresis data for polycrystalline 1-Dy from 2.0 to $4.0 \mathrm{~K}$ at a sweep rate of $82(2) \mathrm{Oe} / \mathrm{s}$ for $|H|>10 \mathrm{kOe}$ and $24(1) \mathrm{Oe} / \mathrm{s}$ for $|H|<10 \mathrm{kOe}$. Only data from $-10 \mathrm{kOe}$ to $10 \mathrm{kOe}$ are shown in order to allow $H_{\mathrm{c}}$ to be visualized more clearly (see main text for full data).

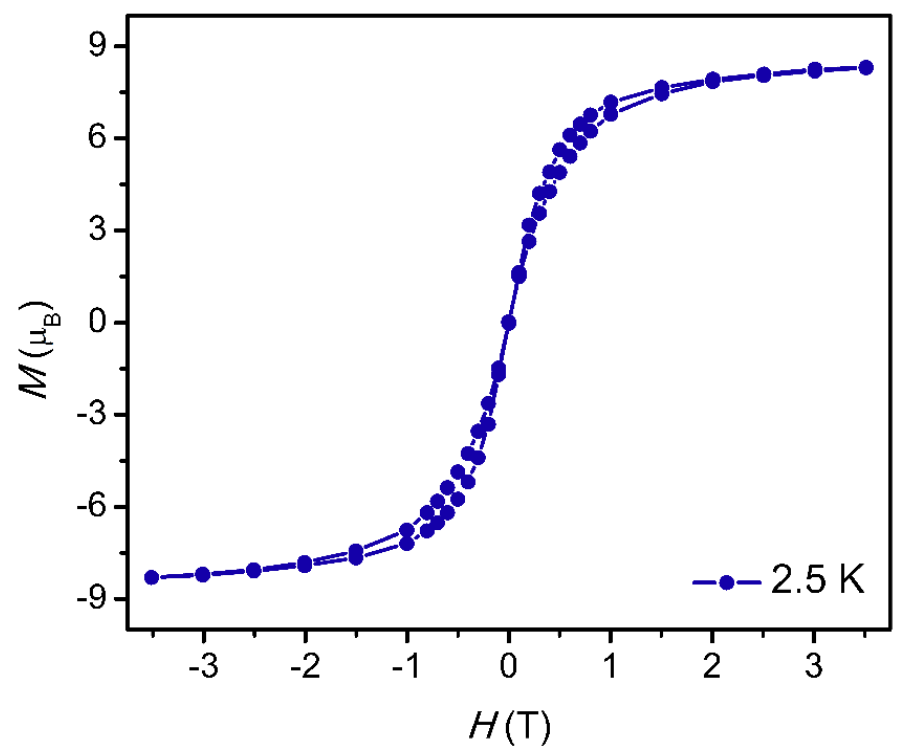

Figure S88. Magnetic hysteresis data for polycrystalline 1-Tb at $2.5 \mathrm{~K}$ at a sweep rate of 82(2) Oe/s for $|H|>10 \mathrm{kOe}$ and 24(1) Oe/s for $|H|<10 \mathrm{kOe}$. 


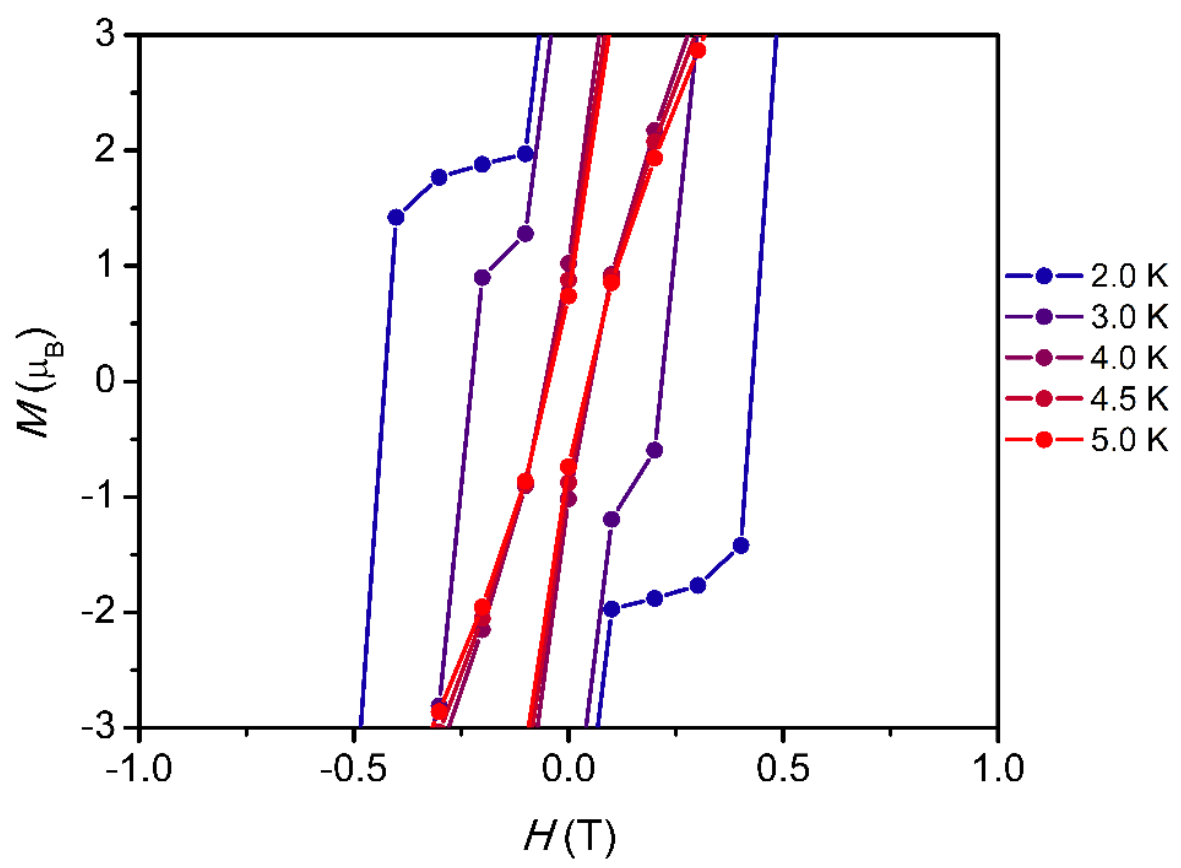

Figure S89. Magnetic hysteresis data for polycrystalline 2-Dy from 2.0 to $5.0 \mathrm{~K}$ at a sweep rate of $82(2) \mathrm{Oe} / \mathrm{s}$ for $|H|>10 \mathrm{kOe}$ and 24(1) Oe/s for $|H|<10 \mathrm{kOe}$. Only data from $-10 \mathrm{kOe}$ to $10 \mathrm{kOe}$ are shown in order to allow $H_{\mathrm{c}}$ to be visualized more clearly (see main text for full data).

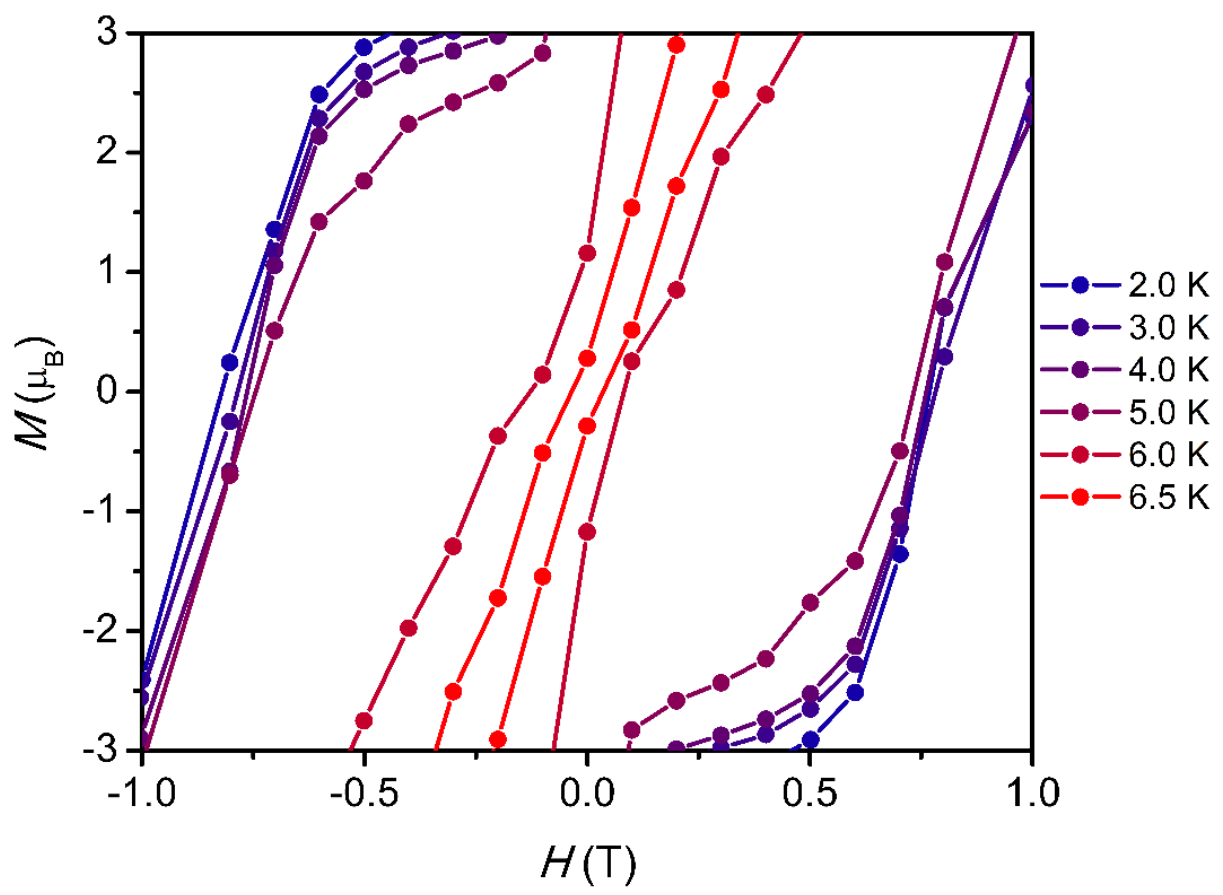

Figure S90. Magnetic hysteresis data for polycrystalline 3-Dy from 2.0 to $6.5 \mathrm{~K}$ at a sweep rate of $82(2) \mathrm{Oe} / \mathrm{s}$ for $|H|>10 \mathrm{kOe}$ and $24(1) \mathrm{Oe} / \mathrm{s}$ for $|H|<10 \mathrm{kOe}$. Only data from $-10 \mathrm{kOe}$ to $10 \mathrm{kOe}$ are shown in order to allow $H_{\mathrm{c}}$ to be visualized more clearly (see main text for full data). 


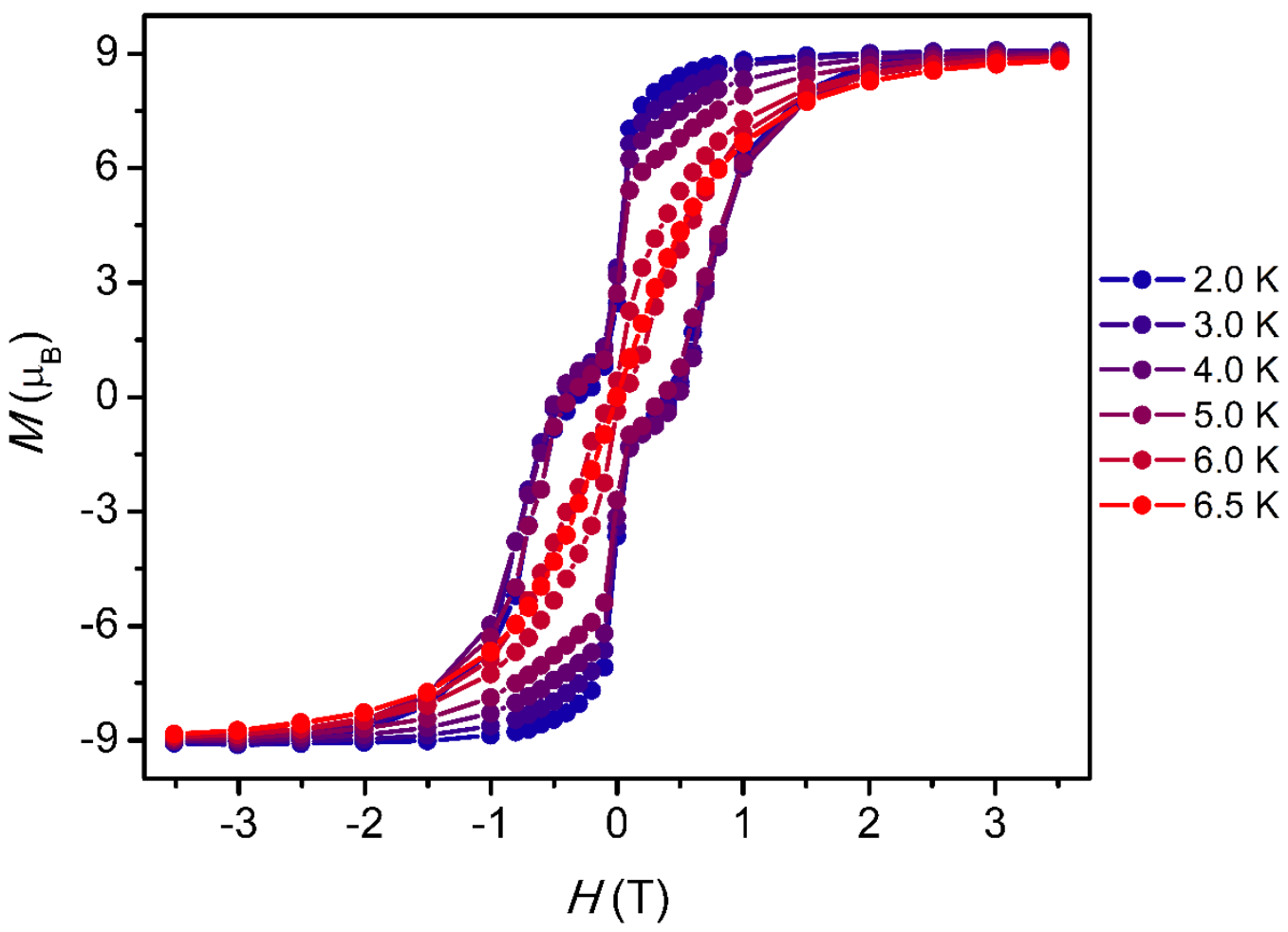

Figure S91. Magnetic hysteresis data for a 7.8 mM solution of 3-Dy from 2.0 to $6.5 \mathrm{~K}$ at a sweep rate of 82(2) Oe/s for $|H|>10 \mathrm{kOe}$ and 24(1) Oe/s for $|H|<10 \mathrm{kOe}$.

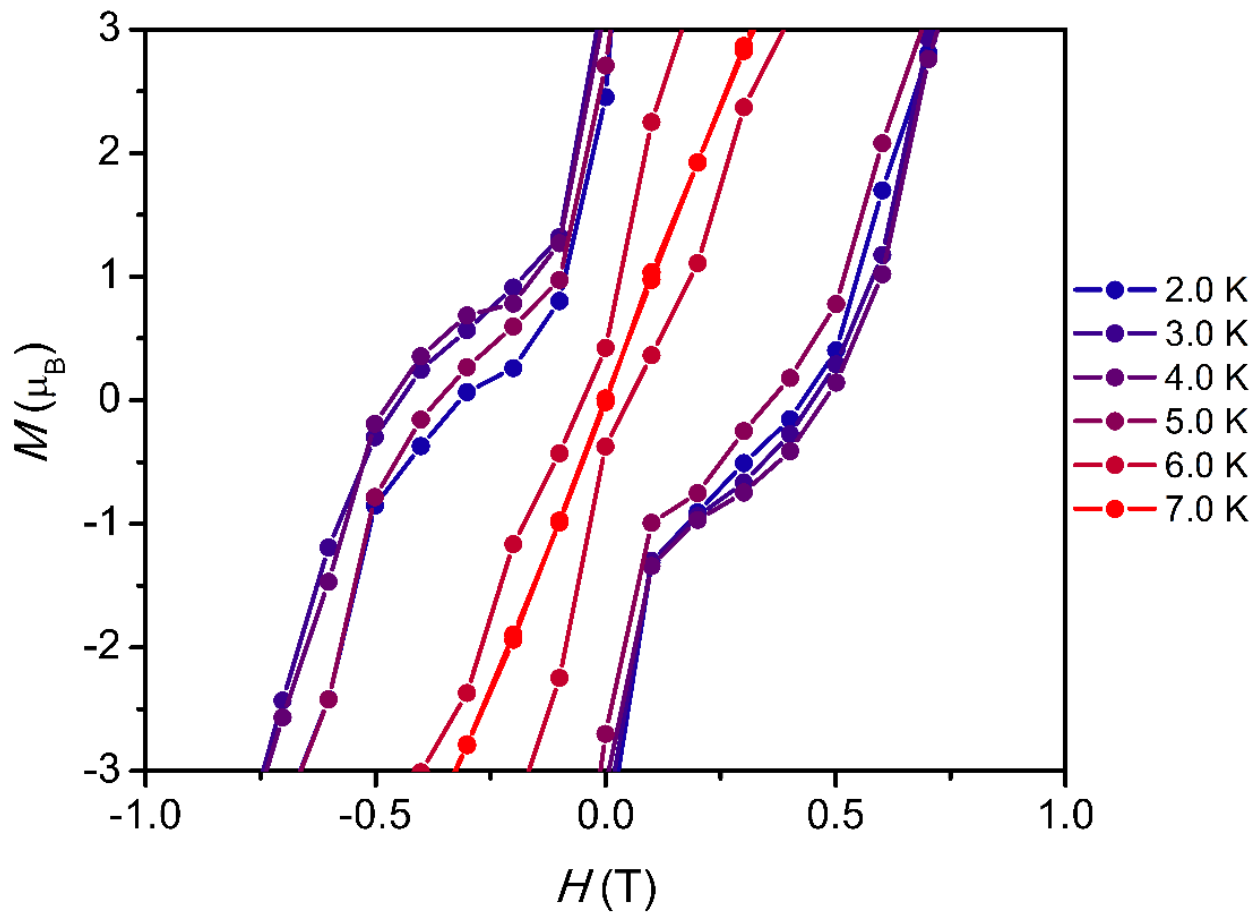

Figure S92. Magnetic hysteresis data for a $7.8 \mathrm{mM}$ solution of 3-Dy from 2.0 to $7.0 \mathrm{~K}$ at a sweep rate of $82(2) \mathrm{Oe} / \mathrm{s}$ for $|H|>10 \mathrm{kOe}$ and $24(1) \mathrm{Oe} / \mathrm{s}$ for $|H|<10 \mathrm{kOe}$. Only data from $-10 \mathrm{kOe}$ to 10 kOe are shown in order to allow $H_{\mathrm{c}}$ to be visualized more clearly (see above for full data). 


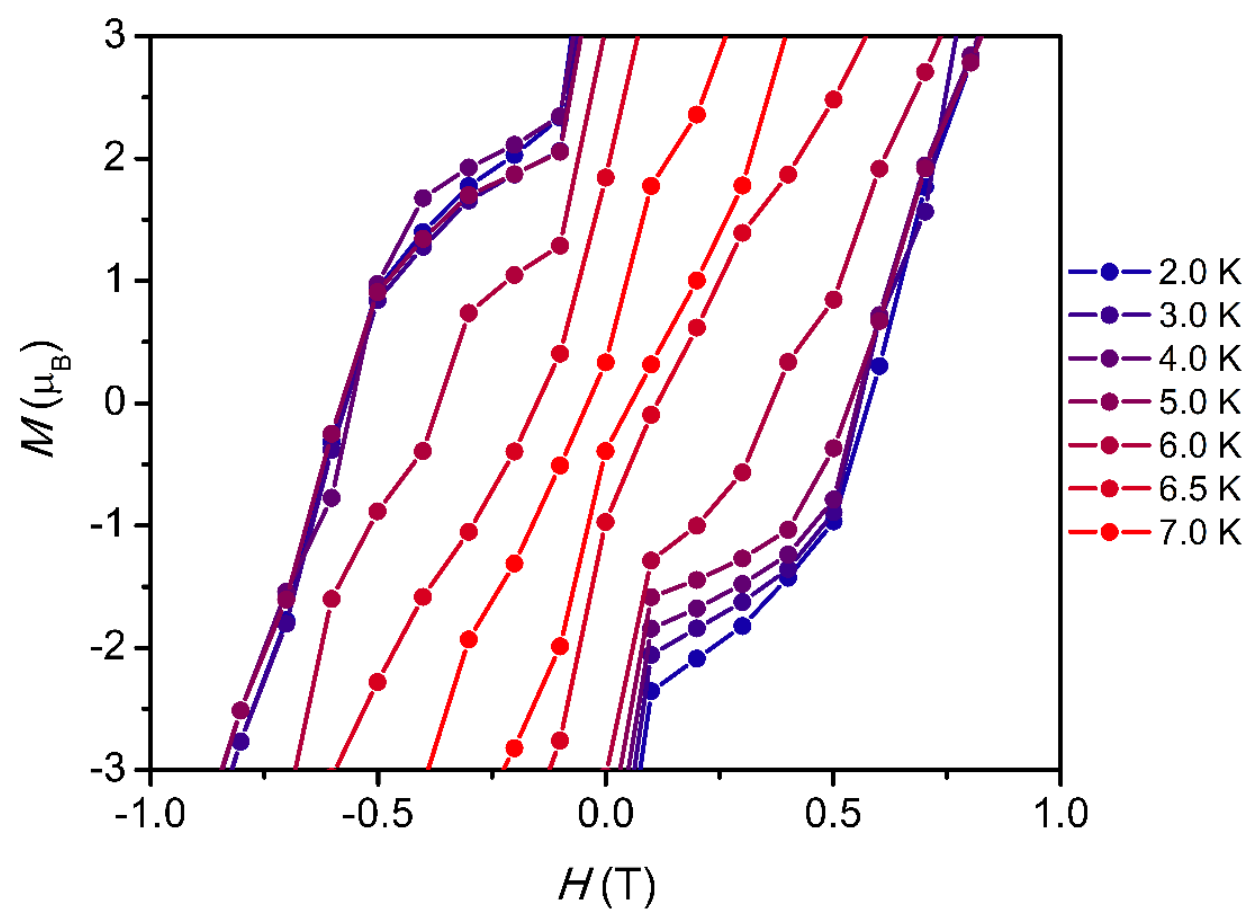

Figure S93. Magnetic hysteresis data for polycrystalline 4-Dy from 2.0 to $7.0 \mathrm{~K}$ at a sweep rate of $82(2) \mathrm{Oe} / \mathrm{s}$ for $|H|>10 \mathrm{kOe}$ and 24(1) Oe/s for $|H|<10 \mathrm{kOe}$. Only data from $-10 \mathrm{kOe}$ to $10 \mathrm{kOe}$ are shown in order to allow $H_{\mathrm{c}}$ to be visualized more clearly (see main text for full data).

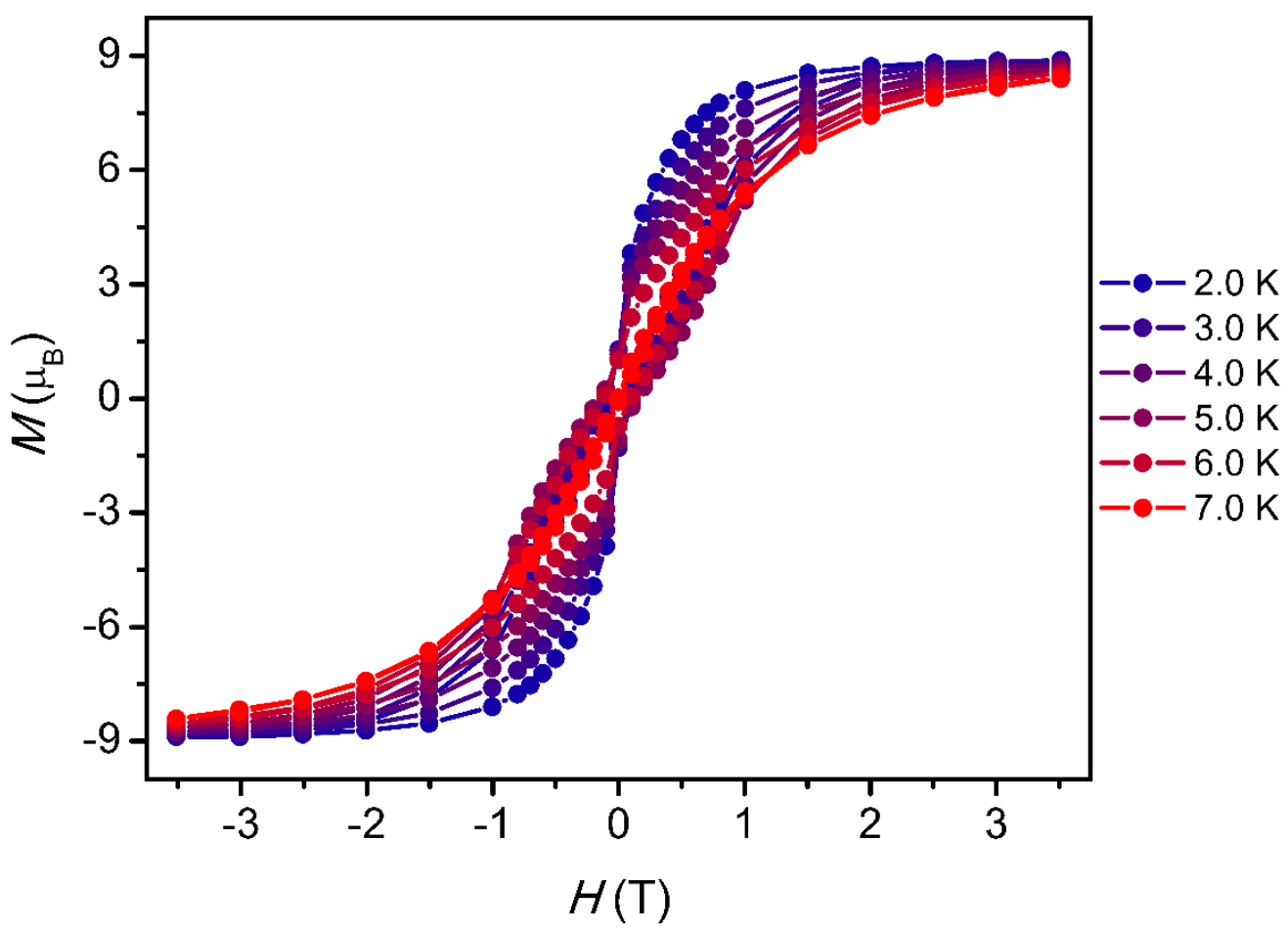

Figure S94. Magnetic hysteresis data for an $8.0 \mathrm{mM}$ solution of 4-Dy from 2.0 to $7.0 \mathrm{~K}$ at a sweep rate of 82(2) Oe/s for $|H|>10 \mathrm{kOe}$ and 24(1) Oe/s for $|H|<10 \mathrm{kOe}$. 


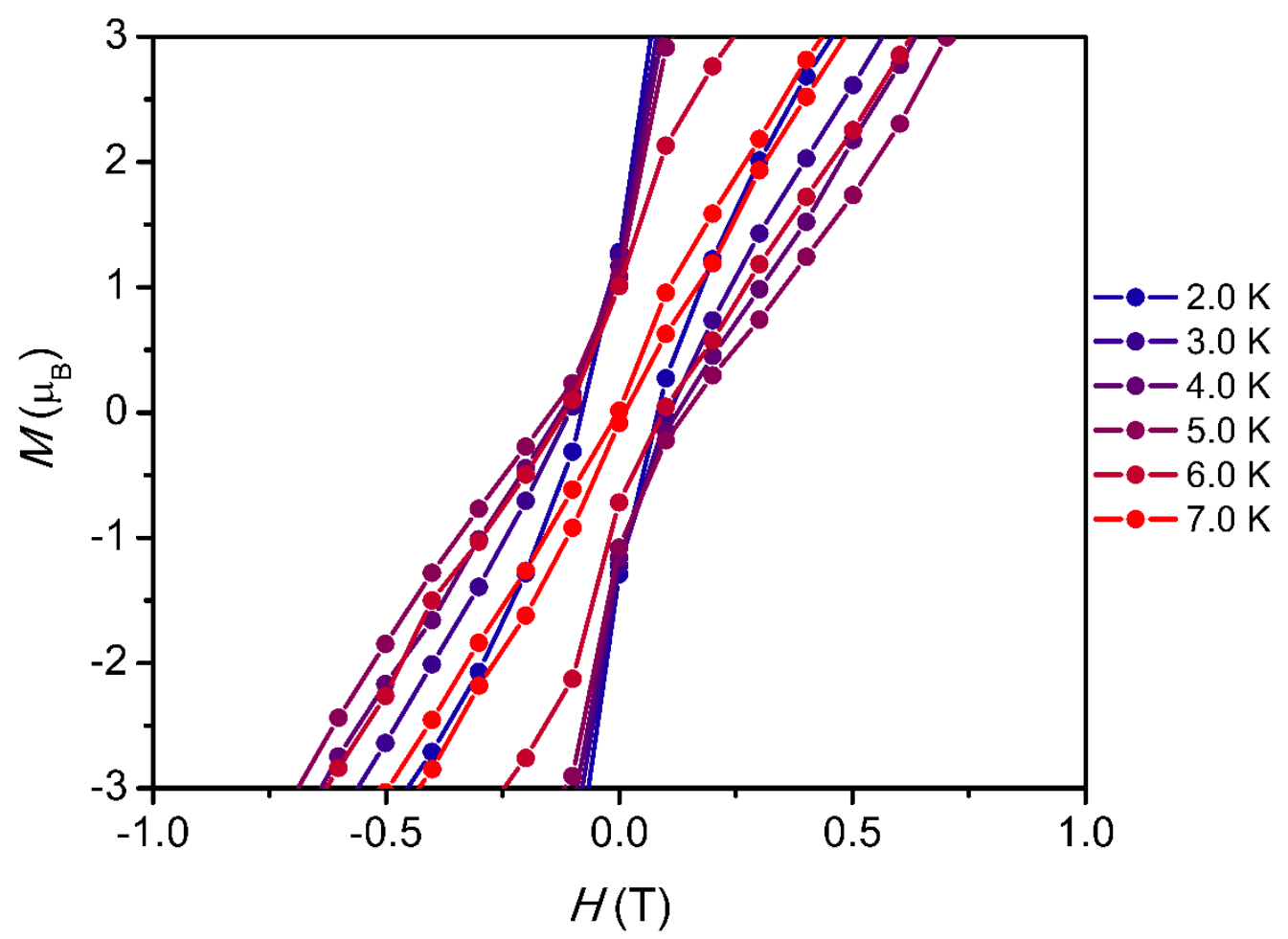

Figure S95. Magnetic hysteresis data for an $8.0 \mathrm{mM}$ solution of 4-Dy from 2.0 to $7.0 \mathrm{~K}$ at a sweep rate of 82(2) Oe/s for $|H|>10 \mathrm{kOe}$ and 24(1) Oe/s for $|H|<10 \mathrm{kOe}$. Only data from $-10 \mathrm{kOe}$ to 10 kOe are shown in order to allow $H_{\mathrm{c}}$ to be visualized more clearly (see above for full data).

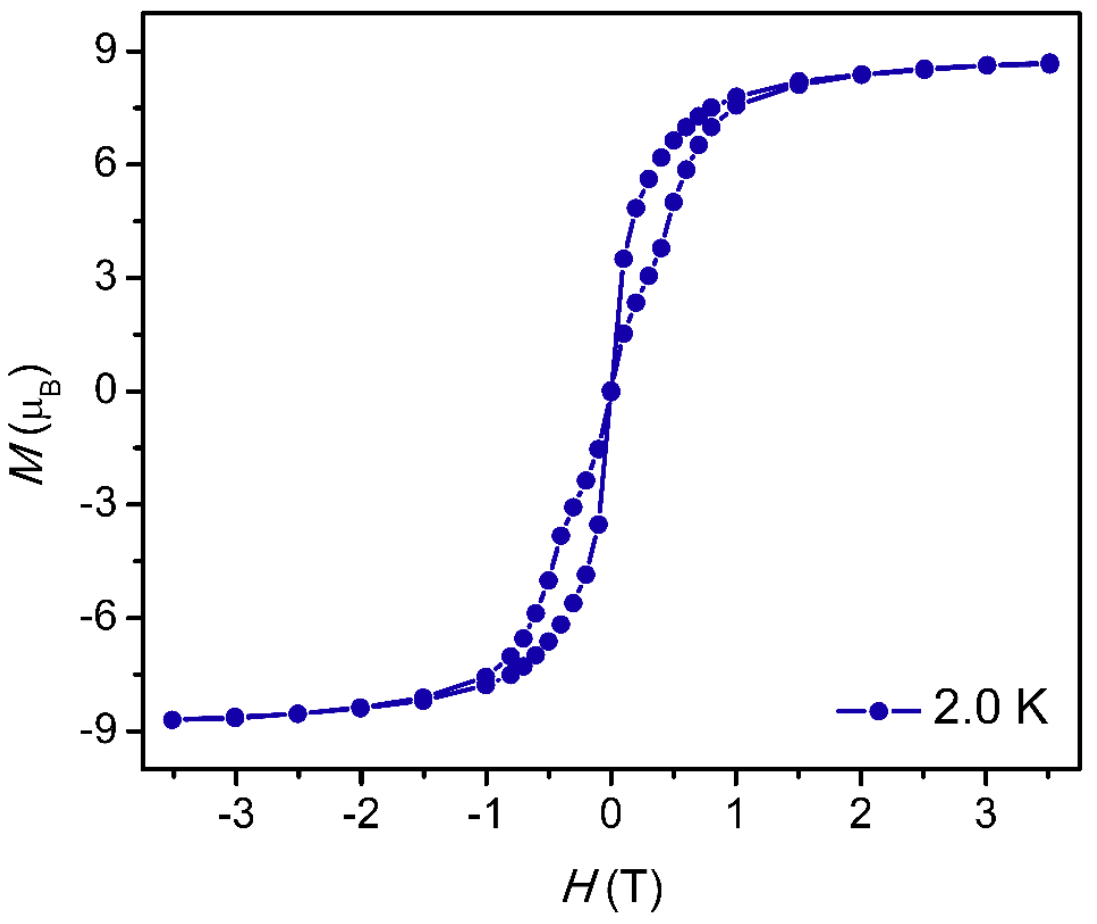

Figure S96. Magnetic hysteresis data for polycrystalline 4-Tb at $2.0 \mathrm{~K}$ at a sweep rate of 82(2) Oe/s for $|H|>10 \mathrm{kOe}$ and 24(1) Oe/s for $|H|<10 \mathrm{kOe}$. 


\section{Section S6. Calculations}

Table S22. Employed basis set.

\begin{tabular}{l} 
Dy: ANO-RCC-VTZP \\
Lu: ANO-RCC-VDZ \\
C \& N (closest): C.ANO-RCC-VDZP \\
O, F, H, N \& C (distant): N.ANO-RCC-VDZ \\
\hline
\end{tabular}

Calculated local crystal field levels of the $\mathrm{Dy}^{3+}$ ions in 1-Dy through 4-Dy are shown in Table S23. For comparison reasons, crystal field levels of $\mathrm{Dy}^{3+}$ ion from $\mathrm{Dy}_{2} \mathrm{~N}_{2}{ }^{3-}$ complex ${ }^{4,11}$ are shown as well. Calculated local crystal field levels of the $\mathrm{Dy}^{3+}$ ions in $\left[\left(\mathrm{Cp}_{2}{ }_{2} \mathrm{Dy}\right)_{2}(\mu \text {-ind })\right]^{-}$are also shown in Table S23. These calculations show that the ground Kramers doublet (KD) in 1-Dy through 4Dy is well separated from the excited states and that the first excited states in these complexes are higher in energy than in the $\mathrm{N}_{2}{ }^{3-}$-bridged complex. By contrast, the ground Kramers doublet in $\left[\left(\mathrm{Cp}{ }_{2} \mathrm{Dy}\right)_{2}(\mu \text {-ind })\right]^{-}$is much less well separated from the first excited state.

Table S23. Energy $\left(\mathrm{cm}^{-1}\right)$ of low-lying Kramers doublets of individual Dy centers in 1-Dy through 4-Dy, $\left[\left(\mathrm{Cp}_{2}{ }_{2} \mathrm{Dy}\right)_{2}(\mu \text {-ind })\right]^{-}$, and $\left[\left(\left\{\mathrm{N}\left(\mathrm{SiMe}_{3}\right)_{2}\right\}_{2}(\mathrm{THF}) \mathrm{Dy}\right)_{2}\left(\mu-\mathrm{N}_{2}\right)\right]^{-}$, and the g-tensor of the corresponding ground doublets as determined by ab initio calculations.

\begin{tabular}{|c|c|c|c|c|c|c|c|}
\hline & 1-Dy ${ }^{a}$ & & & 3-Dy ${ }^{a}$ & 4-Dy ${ }^{a}$ & {$\left[\mathrm{Dy}_{\mathbf{2}}(\boldsymbol{\mu} \boldsymbol{\mu - i n d})\right]^{-a}$} & {$\left[\mathrm{Dy}_{\mathbf{2}}\left(\boldsymbol{\mu}-\mathrm{N}_{2}\right)\right]^{-4, \mathrm{a}}$} \\
\hline & & Dy(1) & Dy(2) & & & & \\
\hline & 0 & 0 & 0 & 0 & 0 & 0 & 0 \\
\hline & 216 & 225 & 238 & 221 & 238 & 71 & 179 \\
\hline & 327 & 347 & 374 & 332 & 374 & 107 & 321 \\
\hline & 377 & 400 & 416 & 385 & 416 & 179 & 407 \\
\hline & 443 & 472 & 481 & 454 & 481 & 209 & 471 \\
\hline & 492 & 530 & 532 & 505 & 532 & 262 & 532 \\
\hline & 596 & 641 & 620 & 611 & 620 & 345 & 623 \\
\hline & 911 & 964 & 923 & 927 & 923 & 576 & 750 \\
\hline$g_{x}$ & 0.00666 & 0.00754 & 0.00960 & 0.00757 & 0.00437 & 0.0282 & 0.0026 \\
\hline $\mathrm{g}_{\mathrm{y}}$ & 0.0141 & 0.01481 & 0.01857 & 0.01519 & 0.00886 & 0.0882 & 0.0040 \\
\hline $\mathrm{g}_{\mathrm{z}}$ & 19.598 & 19.60265 & 19.60576 & 19.60441 & 19.69793 & 18.8953 & 19.6459 \\
\hline
\end{tabular}

${ }^{a}$ This molecule possesses inversion symmetry such that $\mathrm{Dy}(1)=\mathrm{Dy}(2)$

The BS-DFT calculated values for $J_{\mathrm{Gd}-\mathrm{rad}}$ using the X-ray structure of 2-Dy through 4-Dy are -9.8 , -10.6 , and $-10.8 \mathrm{~cm}^{-1}$ respectively. By rescaling these data to the spin $5 / 2$ of $\mathrm{Dy}^{3+}$ ions, one obtains estimated $J_{\text {Lines }}\left(\right.$ Dy-R) constants of $-19.1,-20.7$ and $-21.1 \mathrm{~cm}^{-1}$ for 2-Dy through 4-Dy, respectively. Due to the high axiality of the ground Kramers doublet of the Dy ions (Table S23, $\mathrm{g}_{\mathrm{x}}, \mathrm{g}_{\mathrm{y}}<<\mathrm{g}_{\mathrm{z}}$ ) in 1-Dy through 4-Dy, the low-lying exchange states can be described within the Ising model. This is further justified given the weaker exchange interaction and higher CF levels on the $\mathrm{Dy}^{3+}$ sites of 1-Dy through 4-Dy as compared to the $\mathrm{N}_{2}{ }^{3-}$-bridged complex. According to the Ising model:

$$
\widehat{H}_{\text {exch }}=-J_{D y-R}\left(\tilde{S}_{D y 1} \cdot \hat{S}_{R}+\tilde{S}_{D y 2} \cdot \hat{S}_{R}\right)
$$

where $\tilde{s}_{D y}$ is the pseudospin $1 / 2$ corresponding to the ground Kramers doublet of the Dy ${ }^{3+}$ ion. The BS-DFT extracted values were used as the starting point for the fitting of experimental magnetic 
susceptibility data and the best fit (Figures S97-S100) is given by the exchange parameters from Table S24.

Table S24. Fitted Lines and Ising ${ }^{a}$ exchange parameters for 1-Dy through 4-Dy $\left(\mathrm{cm}^{-1}\right)$.

\begin{tabular}{|c|c|c|c|}
\hline Complex & $\boldsymbol{J}_{\text {Lines }}(\mathbf{D y}-\mathbf{R})$ & $\boldsymbol{J}_{\text {Ising }}(\mathbf{D y}-\mathbf{R})$ & $\begin{array}{c}\boldsymbol{z} \boldsymbol{J}^{\prime} \text { (intermolecular } \\
\text { interaction) }\end{array}$ \\
\hline 1-Dy & -19.0 & -95.0 & -0.15 \\
\hline 2-Dy & -24.0 & -120.0 & -0.08 \\
\hline 3-Dy & -30.0 & -150.0 & -0.03 \\
\hline 4-Dy & -34.0 & -170.0 & -0.12 \\
\hline
\end{tabular}

${ }^{a}$ In the current case, Ising exchange parameters can be obtained by a simple rescaling of the Lines parameters, $J_{\text {Ising }}=5 J_{\text {Lines. }}$.

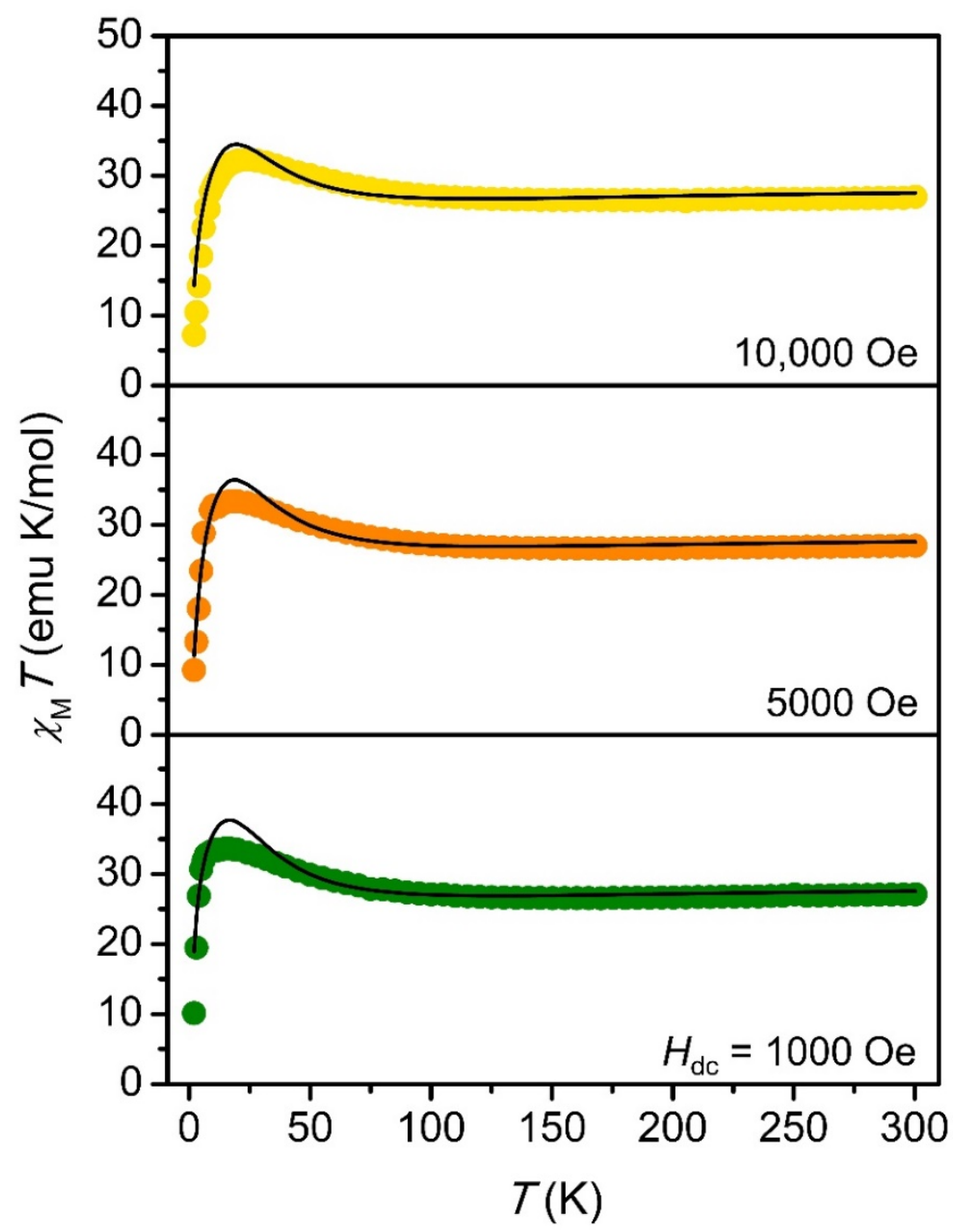

Figure S97. Zero-field cooled dc magnetic susceptibility measurements of 1-Dy under applied fields of 1,5 , and $10 \mathrm{kOe}$. Black lines represent calculated fits to the data used to extract $J_{\text {Lines. }}$ 


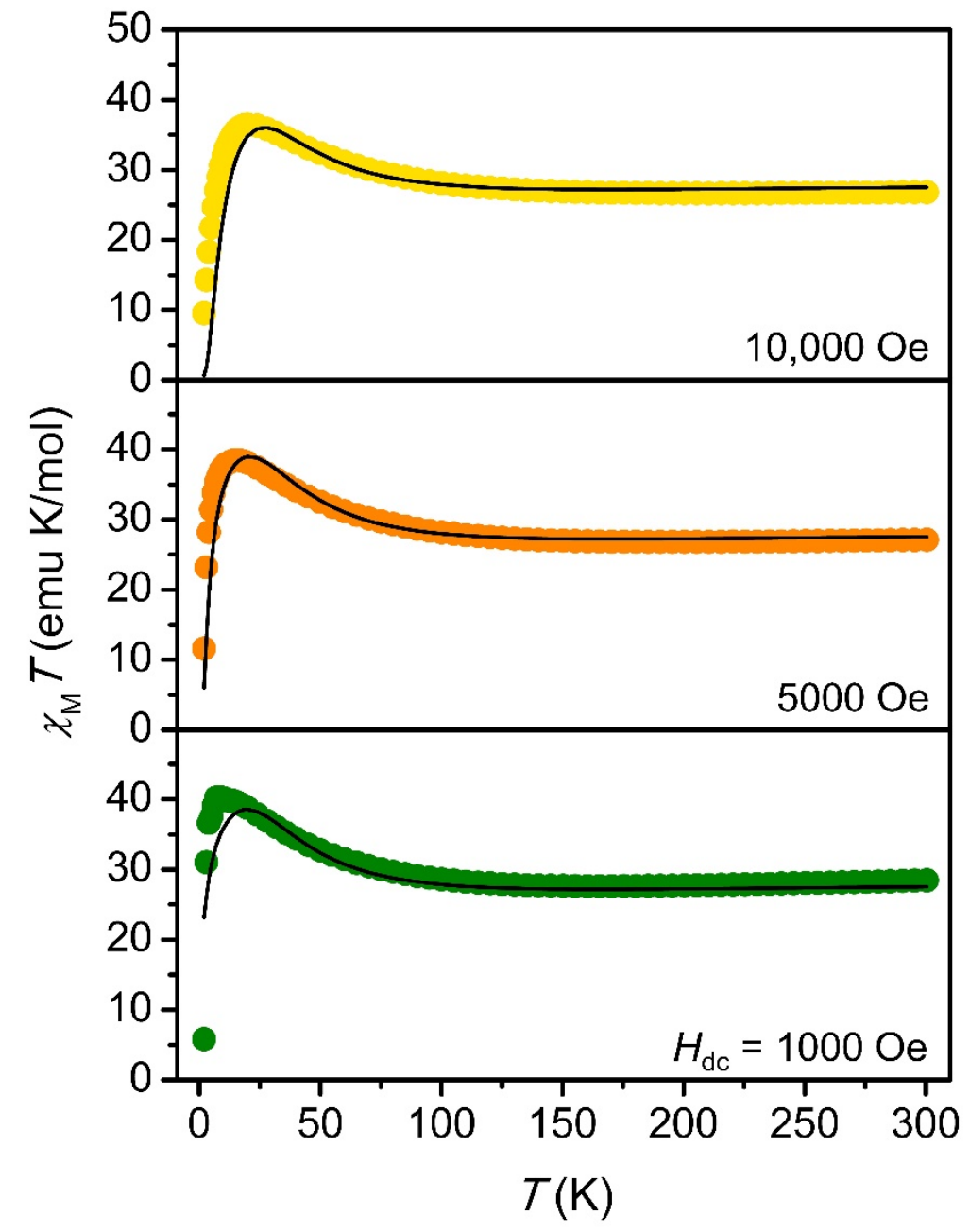

Figure S98. Zero-field cooled dc magnetic susceptibility measurements of 2-Dy under applied fields of 1,5 , and $10 \mathrm{kOe}$. Black lines represent calculated fits to the data used to extract $J_{\text {Lines. }}$ 


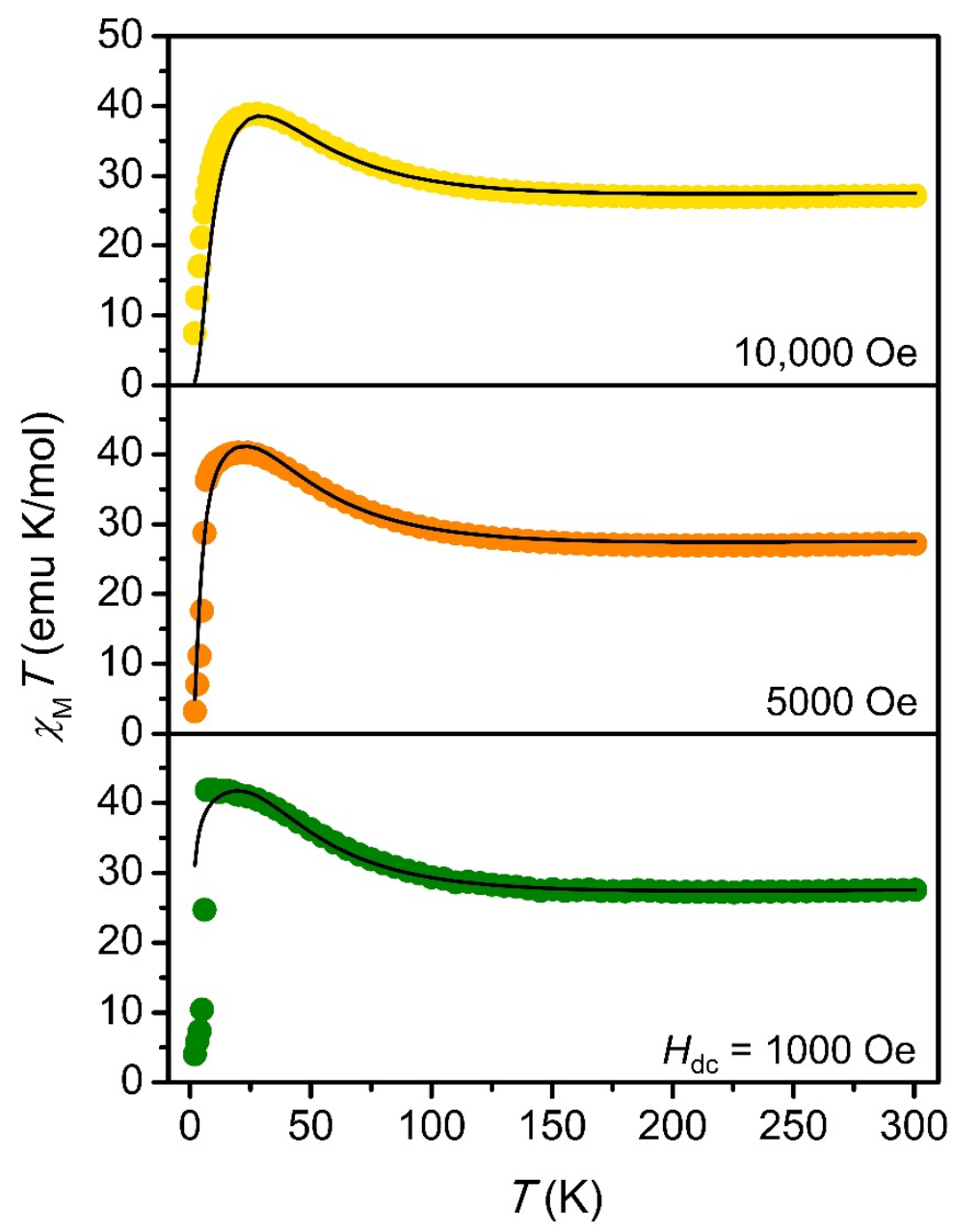

Figure S99. Zero-field cooled dc magnetic susceptibility measurements of 3-Dy under applied fields of 1,5 , and $10 \mathrm{kOe}$. Black lines represent calculated fits to the data used to extract $J_{\text {Lines. }}$ The experimental curves were upscaled by $3 \%$. 


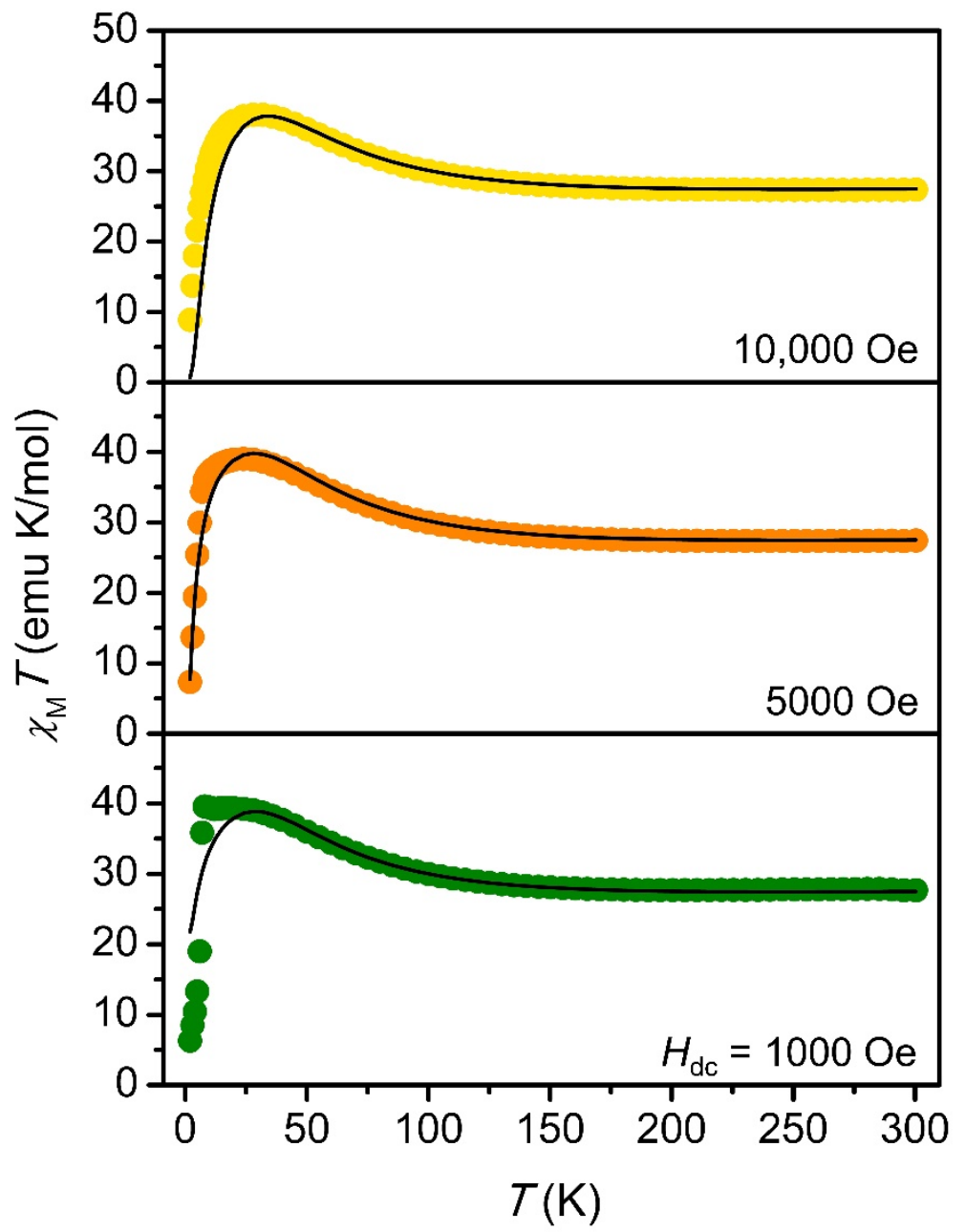

Figure S100. Zero-field cooled dc magnetic susceptibility measurements of 4-Dy under applied fields of 1,5 , and $10 \mathrm{kOe}$. Black lines represent calculated fits to the data used to extract $J_{\text {Lines. The }}$ experimental curves were upscaled by $5 \%$. 
The calculated exchange spectra for 1-Dy through 4-Dy are shown in Table S25 and Figure 4 in the main text. ${ }^{27}$ One can see that the quantum tunneling of magnetization in the ground exchange doublet is small and the relaxation of magnetization would occur via first/second excited doublet in the domain of low temperature, whereas at higher $T$ the relaxation will mainly go via third excited state, which is twice higher in energy. See the main text for further explanation.

Table S25. Calculated exchange energy spectrum $\left(\mathrm{cm}^{-1}\right)$ for 1-Dy through 4-Dy calculated with the parameters from Table S24.

\begin{tabular}{|c|c|c|c|}
\hline 1-Dy & 2-Dy & 3-Dy & 4-Dy \\
\hline 0.00000 & 0.00000 & 0.00000 & 0.00000 \\
0.00000 & 0.00000 & 0.00000 & 0.00000 \\
46.18058 & 56.75684 & 72.50960 & 82.52796 \\
46.18058 & 56.75684 & 72.50960 & 82.52796 \\
46.18059 & 59.22877 & 72.50966 & 82.52798 \\
46.18059 & 59.22877 & 72.50966 & 82.52798 \\
92.78604 & 115.98551 & 145.01916 & 165.05590 \\
92.78604 & 115.98551 & 145.01916 & 165.05590 \\
\hline
\end{tabular}




\section{Section S7. References}

(1) Reta, D.; Chilton, N. F. Uncertainty estimates for magnetic relaxation times and magnetic relaxation parameters. Phys. Chem. Chem. Phys. 2019, 21, 23567-23575.

(2) Evans, P.; Reta, D.; Whitehead, G. F. S.; Chilton, N. F.; Mills, D. P. Bis-Monophospholyl Dysprosium Cation Showing Magnetic Hysteresis at 48 K. J. Am. Chem. Soc. 2019, 141, 19935-19940.

(3) Demir, S.; Jeon, I. R.; Long, J. R.; Harris, T. D. Radical ligand-containing single-molecule magnets. Coord. Chem. Rev. 2015, 289-290, 149-176.

(4) Vieru, V.; Iwahara, N.; Ungur, L.; Chibotaru, L. F. Giant exchange interaction in mixed lanthanides. Sci. Rep. 2016, 6, 24046.

(5) Demir, S.; Zadrozny, J. M.; Nippe, M.; Long, J. R. Exchange Coupling and Magnetic Blocking in Bipyrimidyl Radical-Bridged Dilanthanide Complexes. J. Am. Chem. Soc. 2012, 134, 18546-18549.

(6) Gould, C. A.; Darago, L. E.; Gonzalez, M. I.; Demir, S.; Long, J. R. A Trinuclear RadicalBridged Lanthanide Single-Molecule Magnet. Angew. Chem. Int. Ed. 2017, 56, 1010310107

(7) Zhang, P.; Perfetti, M.; Kern, M.; Hallmen, P. P.; Ungur, L.; Lenz, S.; Ringenberg, M. R.; Frey, W.; Stoll, H.; Rauhut, G.; van Slageren, J. Exchange coupling and single molecule magnetism in redox-active tetraoxolene-bridged dilanthanide complexes. Chem. Sci. 2018, 9, 1221-1230.

(8) Demir, S.; Nippe, M.; Gonzalez, M. I.; Long, J. R. Exchange coupling and magnetic blocking in dilanthanide complexes bridged by the multi-electron redox active ligand 2,3,5,6-tetra(2-pyridyl)pyrazine. Chem. Sci. 2014, 5, 4701-4711.

(9) Guo, F.-S.; Layfield, R. A. Strong direct exchange coupling and single-molecule magnetism in indigo-bridged lanthanide dimers. Chem. Comm. 2017, 53, 3130-3133.

(10) Demir, S.; Gonzalez, M. I.; Darago, L. E.; Evans, W. J.; Long, J. R. Giant coercivity and high magnetic blocking temperatures for $\mathrm{N}_{2}{ }^{3-}$ radical-bridged dilanthanide complexes upon ligand dissociation. Nat. Commun. 2017, 8, 2144.

(11) Rinehart, J. D.; Fang, M.; Evans, W. J.; Long, J. R. Strong exchange and magnetic blocking in $\mathrm{N}_{2}{ }^{3-}$-radical-bridged lanthanide complexes. Nat. Chem. 2011, 3, 538-542.

(12) Benelli, C.; Caneschi, A.; Gatteschi, D.; Pardi, L. Gadolinium(III) complexes with pyridine-substituted nitronyl nitroxide radicals. Inorg. Chem. 1992, 31, 741-746.

(13) Poneti, G.; Bernot, K.; Bogani, L.; Caneschi, A.; Sessoli, R.; Wernsdorfer, W.; Gatteschi, D. A rational approach to the modulation of the dynamics of the magnetization in a dysprosium-nitronyl-nitroxide radical complex. Chem. Commun. 2007, 18, 1807-1809.

(14) Mei, X.-L.; Ma, Y.; Li, L.-C.; Liao, D.-Z. Ligand field-tuned single-molecule magnet behavior of 2p-4f complexes. Dalton Trans. 2012, 41, 505-511.

(15) Mei, X.-L.; Liu, R.-N.; Wang, C.; Yang, P.-P.; Li, C.-L.; Liao, D.-Z. Modulating spin dynamics of cyclic $\mathrm{Ln}^{\mathrm{III}}$-radical complexes $\left(\mathrm{Ln}^{\mathrm{III}}=\mathrm{Tb}\right.$, Dy) by using phenyltrifluoroacetylacetonate coligand.

(16) Mei, X.; Wang, X.; Wang, J.; Ma, Y.; Li, L.; Liao, D. Dinuclear lanthanide complexes bridged by nitronyl nitroxide radical ligands with 2-phenolate groups: structure and magnetic properties. New J. Chem. 2013, 37, 3620-3626.

(17) Liu, R.; Zhang, C.; Li, L.; Liao, D.; Sutter, J.-P. Ligand substitution effect on singlemolecule magnet behavior in dinuclear dysprosium complexes with radical functionalized phenol as bridging ligands. Dalton Trans. 2012, 41, 12139-12144. 
(18) Hu, P.; Zhu, M.; Mei, X.; Tian, H.; Ma, Y.; Li, L.; Liao, D. Single-molecule magnets based on rare earth complexes with chelating benzimidazole-substituted nitronyl nitroxide radicals. Dalton Trans. 2012, 41, 14651-14656.

(19) Rinehart, J. D.; Fang, M.; Evans, W. J.; Long, J. R. A N $2^{3-}$ Radical-Bridged Terbium Complex Exhibiting Magnetic Hysteresis at 14 K. J. Am. Chem. Soc. 2011, 133, 1423614239.

(20) Zhou, N.; Ma, Y.; Wang, C.; Xu, G.-F.; Tang, J.; Yan, S.-P.; Liao, D.-Z. Two tri-spin complexes based on gadolinium and nitronyl nitroxide radicals: Structure and ferromagnetic interactions. J. Solid State Chem. 2010, 183, 927-932.

(21) Zhou, N.; Ma, Y.; Wang, C.; Xu, G. F.; Tang, J.-K.; Xu, J.-X.; Yan, S.-P.; Cheng, P.; Li, L.-C.; Liao, D.-Z. A monometallic tri-spin single-molecule magnet based on rare earth radicals. Dalton Trans. 2009, 40, 8489-8492.

(22) Benelli, C.; Caneschi, A.; Gatteschi, D.; Pardi, L. Gadolinium(III) complexes with pyridine-substituted nitronyl nitroxide radicals. Inorg. Chem. 1992, 31, 741-746.

(23) Wang, X.-L.; Li, C.-L.; Liao, D.-Z. Slow Magnetic Relaxation in Lanthanide Complexes with Chelating Nitronyl Nitroxide Radical. Inorg. Chem. 2010, 49, 4735-4737.

(24) Pointillart, F.; Bernot, K.; Poneti, G.; Sessoli, R. Crystal Packing Effects on the Magnetic Slow Relaxation of Tb(III)-Nitronyl Nitroxide Radical Cyclic Dinuclear Clusters. Inorg. Chem. 2012, 51, 12218-12229.

(25) Xu, J.-X.; Ma, Y.; Xu, G.-F.; Wang, C.; Liao, D.-Z.; Jiang, Z.-H.; Yan, S.-P.; Li, L.-C. A four-spin ring with alternating magnetic interactions formed by pyridine-substituted nitronyl nitroxide radicals and Gd(III) ions: Crystal structure and magnetic properties. Inorg. Chem. Commun. 2008, 11, 1358-1358.

(26) Xu, J.-X.; Ma, Y.; Liao, D.-Z.; Xu, G.-F.; Tang, J.; Wang, C.; Zhou, N.; Yan, S.-P.; Cheng, P.; Li, L.-C. Four New Lanthanide Nitronyl Nitroxide ( $\left.\mathrm{Ln}^{\mathrm{III}}=\mathrm{Pr}^{\mathrm{III}}, \mathrm{Sm}^{\mathrm{III}}, \mathrm{Eu}^{\mathrm{III}}, \mathrm{Tm}^{\mathrm{III}}\right)$ Complexes and a $\mathrm{Tb}^{\mathrm{III}}$ Complex Exhibiting Single-Molecule Magnet Behavior. Inorg. Chem. 2009, 48, 8890-8896.

(27) Ungur, L.; Thewissen, M.; Costes, J.-P.; Wernsdorfer, W.; Chibotaru, L. F. Interplay of Strongly Anisotropic Metal Ions in Magnetic Blocking of Complexes. Inorg. Chem. 2013, $52,6328-6337$. 